\title{
Revisiting Surface Enhanced Raman Scattering on Flat Metallic Surfaces
}

\author{
A thesis by \\ Stefan Andreas Meyer \\ supervised by Prof. Pablo Etchegoin and Assoc. Prof. Eric Le Ru \\ submitted to the Victoria University of Wellington \\ fulfilling the requirements for the degree of Doctor of Philosophy in Physics
}

Victoria University of Wellington

The MacDiarmid Institute for Advanced Materials \& Nanotechnology

April 2012 


\section{Acknowlegements}

Two years ago, on May $13^{\text {th }}$ 2010, I received an e-mail from Pablo telling us (his group) that he was diagnosed with cancer with odds against him and a very uncertain future ahead. I'll never forget the sadness after my visit in the hospital and the emptiness in the lab the next days. That week was truly the lowest point of my time as PhD student. It is in this context that I feel incredibly lucky and blessed that Pablo defied the odds and is able to read the result of his input and guidance in person. I would like to express to him first and foremost my cordial thanks: for his guidance and the many explanations on the whiteboard; for spending countless hours with me in the lab, showing me the equipment and helping me to acquire the skills I needed for my research; for showing up at Uni every day with a smile on his face and never short of a jovial remark despite the most grueling therapies - the humor and serenity with which he takes his illness is one of the most inspiring things I've witnessed in my life; and for all the funny stories, the political and philosophical discussions and the laughter that made work a fun place to be.

I would also like to extend my cordial thanks to Eric for his patience and encouragement in learning how to program, his help with fitting the data and his countless theoretical explanations. They always seemed so simple and self-evident when he would write them on the board for me. The clear guidance and prompt feedback I received from him was invaluable and made me feel that my work was appreciated.

My friends Peter A. and Felix W. were my moral support during this thesis, always there to listen. They helped me to turn this $\mathrm{PhD}$ into an opportunity not only for scientific but also spiritual growth and I'd like to thank them for their encouragement and non-judgmental ear.

There are my crazy office-mates without whom life in general and work in particular would just seem dull and meaningless. My thanks goes to Chris Galloway (despite exchanging the " $n$ " and the " $\mathrm{m}$ " on my keyboard), Evan Blackie (even though he didn't play soccer), Walter Somerville (for his inexhaustible help with $\mathrm{BT}_{\mathrm{E}} \mathrm{X}$ and computer issues and despite making the movie "Mini Shark versus Tiny Octopus" (MSVTO)), Baptiste Auguié (for the awesome collaboration on the experiments and despite giving me a 32-dimensional Shark puzzle for Christmas), to Camille Arthur (her cheerful presence and organized chaos makes the lab a fun place to be) and Peter Hauer (who finally relieved me from the task of organizing lunch-time soccer :-).

I would like to acknowledge the help I received from the people in the workshop (Manu, Nick and Alain) with the mechanical fabrication of equipment and the MacDiarmid Institute for the PhD scholarship and for providing the excellent resources.

Finally I want to give my cordial thanks to both my parents for their encouragement and financial support during this $\mathrm{PhD}$ endeavour. Especially my father might be able to see in this work the fruit of answering my countless questions about nature as a child instilling in me the love for physics that carried me through the ups and downs of my scientific research.

This work is the result of a collaborative effort and would not have been possible without the help, input, guidance and ideas form Pablo Etchegoin, Eric Le Ru and Baptiste Auguié. Especially for the complex setup required for the second half of Chapter 4 Baptiste's help with the measurements, data-analysis and figures was invaluable.

'The highest form of wisdom is kindness.' Talmud 
Dedicated in gratitude

\section{To Dan, Matthew, Felix and Peter}




\begin{abstract}
Surface enhanced Raman spectroscopy (SERS) is undoubtedly a powerful tool as it allows one to overcome the major disadvantage of Raman spectroscopy: the weakness of its signal. Enhancement factors (EF) of up to $10^{10}$ make it even possible to detect single molecules. However, using it as an analytical tool to make reproducible, quantitative measurements has so far been difficult as the enhancement of the signal is "bought" at the expense of reproducibility: The larger the EF the more the reproducibility of the substrate suffers. This has been dubbed informally the "SERS uncertainty principle" by Natan [1]. While currently a lot of research effort is taking place at the high-EF-side of the spectrum and ever more sophisticated SERS substrates are being explored, in this thesis we would like to make a shift in paradigm and revisit SERS on flat metallic surfaces, which arguably constitute the simplest substrates available. To this end we will show their usefulness in making quantitative measurements and how they are an ideal platform for a new hybrid technique that combines reproducibility and extreme sensitivity with substantial EFs.
\end{abstract}

For making quantitative measurements two examples are explored in a systematic way: in the first example (Chapter 2) the determination of an unknown, resonant Raman cross-section is demonstrated on flat metallic films (possibly with some surface roughness) and confirmed with measurements done on more commonly used SERS substrates. Here the quantitative measurement is made possible by introducing a reference molecule as a standard and having statistics as our main ally: even though we do not know the exact EF that the individual molecules experience on the various substrates, we know that on average both, the unknown sample and the known reference, experience the same. In the second example (Chapter 3) we use commercially available flat films for which we verify experimentally that surface roughness is irrelevant. By themselves these substrates yield no enhancement - in fact they even quench the Raman signal. Yet they allow us to calculate and control the electric field on the surface which enables us to determine the orientation of adsorbed molecules by using surface selection rules (SSR). While the first example is mostly empirical, the second one allows us to test our theoretical understanding of plasmonic systems with proper numerical calculations that are in excellent agreement with the observed data. Finally, in Chapter 4, we use those flat films in a special configuration (called the Kretschmann configuration) to excite Surface Plasmon-Polaritons (SPP). This not only allows us to combine the spatial homogeneity of a flat surface with useful EFs easily predicted from theory but also to combine the extreme sensitivity of surface plasmon resonance spectroscopy (SPRS) with the analytical power of SERS.

It is not our intention to claim that the work presented here is the first attempt to do analytical work with SERS. Rather the new methods presented in this thesis will add new strategies and tools to the current research effort while the detailed analysis will provide the means to understand them theoretically and in their historical context. 


\section{Publications}

The following publications emerged during the course of this thesis:

1. S. A. Meyer, E. C. Le Ru, and P. G. Etchegoin, "Quantifying resonant Raman cross section with SERS," J. Phys. Chem. A, vol. 114, pp. 5515-5519, 2010.

2. E. C. Le Ru, S. A. Meyer, C. Artur, P. G. Etchegoin, J. Grand, P. Lang, and F. Maurel, "Experimental demonstration of surface selection rules for SERS on flat metallic surfaces," Chem. Comm., vol. 47, pp. 3903-3905, 2011.

3. S. A. Meyer, E. C. Le Ru, and P. G. Etchegoin, "Combining surface plasmon resonance (SPR) spectroscopy with surface-enhanced Raman scattering (SERS)," Anal. Chem., vol. 83, pp. 2337$2344,2011$.

4. S. A. Meyer, B. Auguie, E. C. Le Ru, and P. G. Etchegoin, "Combined SPR and SERS microscopy in the Kretschmann configuration,” J. Phys. Chem. A, vol. 116, pp. 1000-1007, 2012. 
Abbreviations used in this thesis (AUITT)

AUITT Abbreviations used in this thesis $(-)$

$\mathrm{Au} \quad$ chemical symbol for gold

Ag chemical symbol for silver

BFP back focal plane

BS back scattering

CV Crystal Violet (dye)

DFT density functional theory

EF enhancement factor

IR infrared

KC Kretschmann configuration

LFEF local field enhancement factor $M_{\text {loc }}$

MSVTO Mini Shark versus Tiny Octopus, in production $\odot$

NA numerical aperture

NB Nile Blue (dye)

RH6G Rhodamine 6G (dye)

RRS resonant Raman scattering

SERS surface enhanced Raman spectroscopy

SMEF single molecule enhancement factor

SP surface plasmon

SPP surface plasmon polariton

SPRS surface plasmon resonance spectroscopy

SPR surface plasmon resonance

SSR surface selection rules

$\mathrm{TM}, \mathrm{TE}$ transverse magnetic (= p-polarization), transverse electric (= s-polarization)

TIRF total internal reflection fluorescence

SPlaC SERS \& Plasmonics Code (see [2], Appendix F)

$\perp, \| \quad$ symbol for perpendicular, parallel 


\section{Notations used in this thesis}

\begin{tabular}{|c|c|c|}
\hline$\stackrel{!}{=}$ & we demand that the left side is equal to the right & e.g. $f(x) \stackrel{!}{=} g(x) \quad \Longrightarrow \quad x=3$ \\
\hline$:=$ & we define that the left side is equal to the right & e.g. $f(x):=3 x^{2}+1$ \\
\hline [ ] & this symbol means "unit of" & example: $[c]=\frac{\mathrm{m}}{\mathrm{s}}$ \\
\hline$\hat{\alpha}$ & Raman tensor & $3 \times 3$ matrix with elements $\in \mathbb{C}$ \\
\hline$\alpha, \alpha^{\mathrm{eff}}$ & $\begin{array}{l}\text { angle between surface normal and beam axis before, } \\
\text { after optical elements }\end{array}$ & {$[\alpha]=\left[\alpha^{\mathrm{eff}}\right]=\operatorname{deg}$} \\
\hline$A$ & absorbance & $A=-\log _{10}(T)$ \\
\hline$c$ & speed of light & $c=2.998 \times 10^{8} \frac{\mathrm{m}}{\mathrm{s}}$ \\
\hline$C_{p}$ & constant used for derivation & $C_{p}=\sqrt{\epsilon_{\mathrm{m}}} \epsilon_{0} c / 2$ \\
\hline$C_{E}$ & constant used for derivation & $C_{E}=\frac{1}{4 \pi \epsilon_{0} c^{2}}$ \\
\hline $\mathbf{e}$ & unit vector & $\mathbf{e} \in \mathbb{R}^{3}$ with $|\mathbf{e}|=1$ \\
\hline 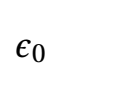 & vacuum permittivity & $\epsilon_{0}=8.854 \times 10^{-8} \frac{\mathrm{A}^{2} \cdot \mathrm{s}^{4}}{\mathrm{~kg} \cdot \mathrm{m}^{3}}$ \\
\hline$\epsilon_{\mathrm{m}}, \epsilon_{\mathrm{d}}$ & dielectric function of metal, dielectric & {$\left[\epsilon_{\mathrm{m}}\right]=1$} \\
\hline$\tilde{\mathbf{E}}(t)$ & electric field & {$[\tilde{\mathbf{E}}(t)]=\frac{\mathrm{V}}{\mathrm{m}}$} \\
\hline $\mathbf{E}(\omega)$ & complex electric field amplitude & $\tilde{\mathbf{E}}(t)=\operatorname{Re}\{\mathbf{E}(\omega) \exp (-i \omega t)\}$ \\
\hline$\hat{\mathbf{E}}_{\text {loc }}$ & $\begin{array}{l}\text { local electric field in the presence of a metal surface } \\
\text { normalized by the electric field strength of the in- } \\
\text { coming light at the same position but without the } \\
\text { metal surface }\end{array}$ & $\hat{\mathbf{E}}_{\text {loc }}:=\frac{\mathbf{E}_{\text {loc }}}{\left|\mathbf{E}_{\text {inc }}\right|}$, see eq. 1.41, p. 17 \\
\hline E & photon energy & $E=h \cdot f=\hbar \cdot \omega$ \\
\hline$f$ & frequency of light & {$[f]=\frac{1}{\mathrm{~s}}$} \\
\hline$F$ & enhancement factor & {$[\mathrm{F}]=1$} \\
\hline$\gamma$ & opening angle of a cone & $\begin{array}{l}\text { example: } \gamma_{\mathrm{Obj}}=\text { opening angle } \\
\text { of objective's collection cone }\end{array}$ \\
\hline$\Gamma$ & decay rate & {$[\Gamma]=\frac{1}{\mathrm{~s}}$} \\
\hline$h$ & Planck's constant & $h=6.626 \times 10^{-34} \mathrm{~J} \cdot \mathrm{s}$ \\
\hline$k_{0}$ & wavenumber of light in vacuum & $k_{0}=\frac{\omega}{c}$ \\
\hline$L_{\mathrm{m}}$ & local field correction factor & $L_{\mathrm{m}}^{1 / 4}=\left(\epsilon_{\mathrm{m}}+2\right) / 3$ \\
\hline . & wavelength & {$[\lambda]=\mathrm{m}$} \\
\hline
\end{tabular}




\section{Notations used in this Text (continued)}

$\begin{array}{lll}M_{\mathrm{loc}} & \text { local field intensity enhancement factor } & M_{\mathrm{loc}}=\left|\hat{\mathbf{E}}_{\mathrm{loc}}\right|^{2} \\ N_{\mathrm{Av}} & \text { Avogadro's constant } & N_{\mathrm{Av}}=6.022 \times 10^{23} \frac{1}{\mathrm{~mol}} \\ n & \text { refractive index } & n=\sqrt{\epsilon_{\mathrm{m}}} \text { for } \epsilon_{\mathrm{m}} \text { real } \\ N A & \text { numerical aperture } & \text { example: } N A_{\mathrm{Obj}}=n \cdot \sin \left(\gamma_{\mathrm{Obj}}\right) \\ \omega & \text { angular frequency of light } & \omega=2 \pi \cdot f \\ \Omega, \Delta \Omega & \text { angle, solid angle } & \Omega=(\theta, \phi),[\Delta \Omega]=\mathrm{sr} \\ P, p & \text { power, power-density } & {[P]=\frac{\mathrm{J}}{\mathrm{s}} \equiv \mathrm{W},[p]=\frac{\mathrm{J}}{\mathrm{s} \cdot \mathrm{m}^{2}}} \\ \mathbf{P}_{\mathrm{L}}, \mathbf{P}_{\mathrm{R}} & \text { unit vectors characterizing polarization in excita- }- & \mathbf{P}_{\mathrm{L}}, \mathbf{P}_{\mathrm{R}} \in \mathbb{R}^{3} \quad \text { with }\left|\mathbf{P}_{\mathrm{L}}\right|=\left|\mathbf{P}_{\mathrm{R}}\right|=1 \\ & \text { tion, detection } & {[\tilde{\mathbf{p}}(t)]=\mathrm{C} \cdot \mathrm{m}} \\ \tilde{\mathbf{p}}(t) & \text { polarization } & \tilde{\mathbf{p}}(t)=\mathrm{Re}\{\mathbf{p}(\omega) \exp (-i \omega t)\} \\ \mathbf{p}(\omega) & \text { complex polarization } & Q=\frac{\Gamma^{\mathrm{rad}}}{\Gamma^{\text {tot }}} \\ Q & \text { fluorescence quantum yield } & \rho=\frac{\mathrm{d}_{\Omega} \sigma_{\perp}}{\mathrm{d}_{\Omega} \sigma_{\|}} \\ \rho & \text { depolarization ratio } & {[\rho]=\frac{1}{\mathrm{~m}^{2}}} \\ \rho & \text { also: molecule density } & {\left[\frac{\mathrm{d} \sigma}{\mathrm{d} \Omega}\right]=\frac{\mathrm{m}^{2}}{\mathrm{sr}}} \\ \mathrm{d}_{\Omega} \sigma \equiv \frac{\mathrm{d} \sigma}{\mathrm{d} \Omega} & \text { differential scattering cross-section } & {[\sigma]=\mathrm{m}^{2}} \\ \sigma & \text { integrated scattering cross-section } & T=\frac{P^{\mathrm{trans}}}{P^{\mathrm{inc}}} \\ T & \text { transmittance } & {[\theta]=\mathrm{deg}} \\ \theta & \text { angle between light ray and surface normal } & \end{array}$




\section{Contents}

1 Introduction 2

1.1 Optical processes: a brief introduction $\ldots \ldots \ldots \ldots \ldots$

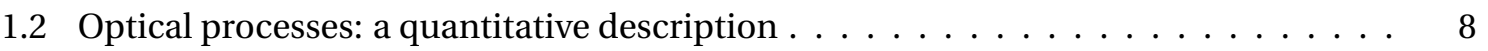

1.2.1 The scattering cross-section $\ldots \ldots \ldots \ldots \ldots \ldots \ldots$

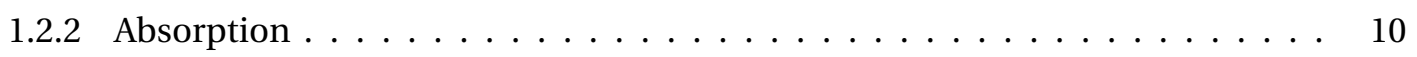

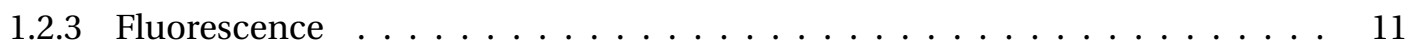

1.2 .4 Rayleigh scattering . . . . . . . . . . . . . . . . . . . . 11

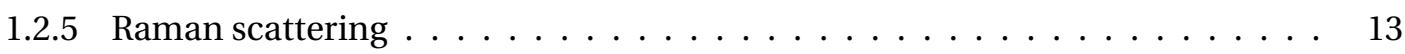

1.2.6 Resonant Raman scattering . . . . . . . . . . . . . . . . . . . . . . 15

1.3 Enhanced Raman and fluorescence $\ldots \ldots \ldots \ldots \ldots \ldots$

1.3.1 Surface enhanced Raman scattering . . . . . . . . . . . . . . . . . . 16

1.3.2 Surface enhanced fluorescence . . . . . . . . . . . . . . . . 19

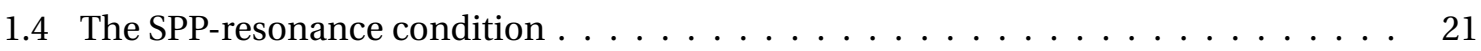

1.4.1 Plane wave solution in a homogeneous medium $\ldots \ldots \ldots \ldots$

1.4.2 Plane wave solution in two homogeneous media . . . . . . . . . . . . . 21

1.4.3 Sign-convention for plane-wave solutions $\ldots \ldots \ldots \ldots \ldots \ldots$

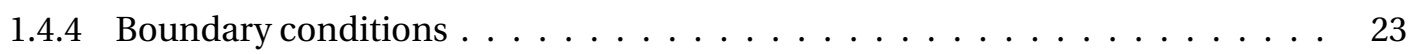

1.4 .5 SPP resonance condition $\ldots \ldots \ldots \ldots \ldots \ldots \ldots \ldots \ldots \ldots$

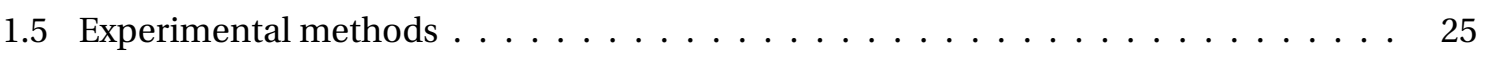

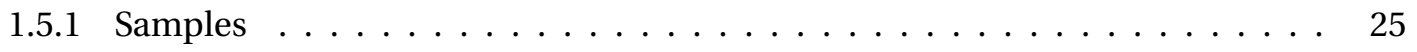

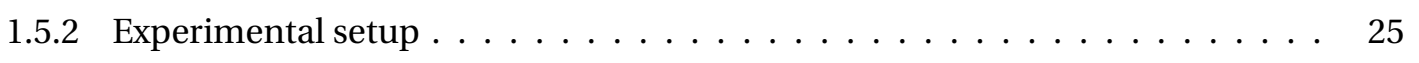


2 Measuring resonant Raman cross-sections $\quad 28$

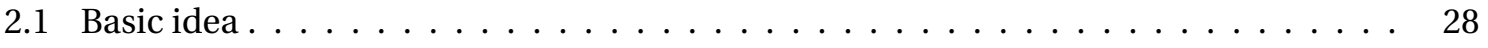

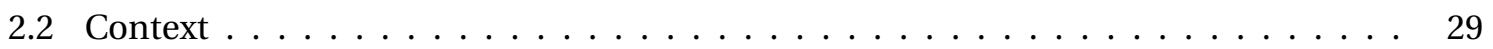

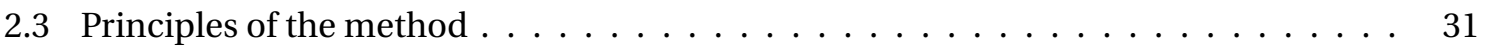

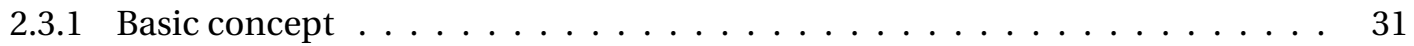

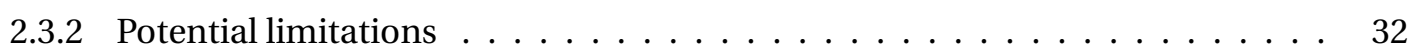

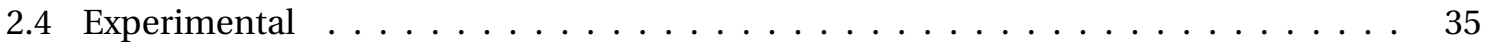

2.4.1 Determination of the reference cross-section $\mathrm{d}_{\Omega} \sigma^{\mathrm{CV}}(\lambda) \ldots \ldots \ldots$

2.4.2 Determination of the relative intensity ratios $R_{i}(\lambda) \ldots \ldots \ldots \ldots$

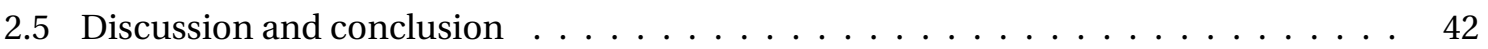

3 Surface selection rules in SERS $\quad 43$

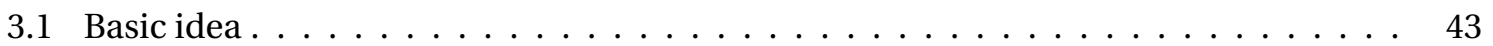

3.2 Context: historical background and relevance $\ldots \ldots \ldots \ldots \ldots$. . . . . . 44

3.3 Theory: the angular emission profile $\ldots \ldots \ldots \ldots \ldots \ldots$

3.3.1 The SERS EF for a uniaxial tensor $\ldots \ldots \ldots \ldots \ldots \ldots$

3.3.2 Numerical evaluation of the SERS EF for a uniaxial tensor . . . . . . . . . . 51

3.3.3 Prediction of SERS intensities for a monolayer of molecules . . . . . . . . . 53

3.3.4 Numerical strategy to evaluate the angular emission profile . . . . . . . . . 56

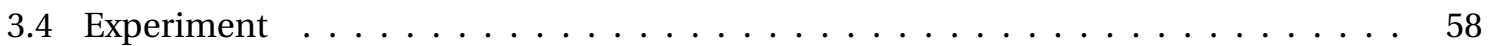

3.4.1 Setup and sample preparation $\ldots \ldots \ldots \ldots \ldots \ldots \ldots$

3.4 .2 EF estimation at normal incidence . . . . . . . . . . . . . . . 59

3.4 .3 Experimental results $\ldots \ldots \ldots \ldots \ldots \ldots \ldots \ldots$

3.4.4 IR absorption and DFT study of the orientation of NB on Au $\ldots \ldots \ldots$

3.5 Discussion and conclusion $\ldots \ldots \ldots \ldots \ldots \ldots$

4 SERS in the Kretschmann configuration $\quad 62$

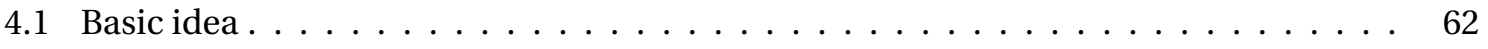

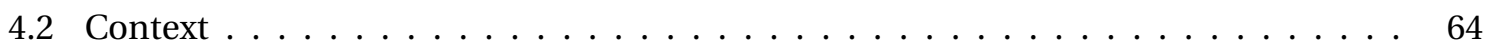

4.2.1 Motivation - a shift in paradigm for SERS substrates $\ldots \ldots \ldots$. . . . . . 64 
4.2 .2 Historical background . . . . . . . . . . . . . . . . . . . . 65

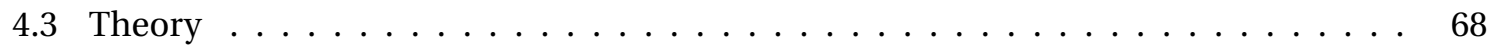

4.3.1 Excitation of SPPs on flat surfaces - the resonance condition $\ldots \ldots$. . . . . 68

4.3.2 Dipole emission on a flat surface - the Kretschmann cone . . . . . . . . . . . 69

4.3.3 Emission profile in the Kretschmann configuration . . . . . . . . . . . . . . 70

4.3.4 EF calculations - a guide for an intuitive understanding $\ldots \ldots \ldots \ldots$

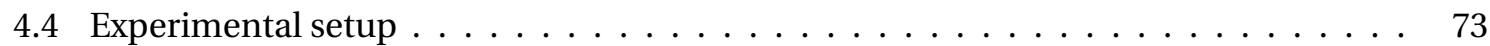

4.5 Combining SPRS with SERS - the prism setup $\ldots \ldots \ldots \ldots \ldots \ldots$

4.5 .1 The physical setup $\ldots \ldots \ldots \ldots \ldots \ldots \ldots \ldots$

4.5 .2 Sample preparation $\ldots \ldots \ldots \ldots \ldots \ldots \ldots \ldots \ldots$

4.5.3 Angle of incidence as a function of sample rotation . . . . . . . . . . . . 77

4.5.4 Modified emission profile for a monolayer of molecules in the prism setup . . 79

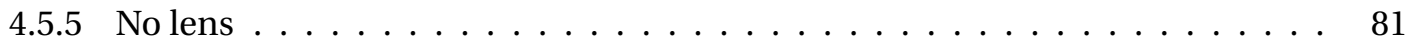

4.5 .6 Collection lens $\ldots \ldots \ldots \ldots \ldots \ldots \ldots \ldots \ldots \ldots$

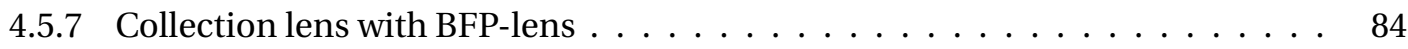

4.6 Collecting the Kretschmann cone - the large NA setup $\ldots \ldots \ldots$. . . . . . . 88

4.6 .1 Experimental setup $\ldots \ldots \ldots \ldots \ldots \ldots \ldots \ldots$

4.6.2 Sample preparation $\ldots \ldots \ldots \ldots \ldots \ldots \ldots \ldots \ldots$

4.6.3 Angle of incidence as a function of cube rotation $\ldots \ldots \ldots$

4.6.4 Trade-off between resolution and coupling to SPP $\ldots \ldots \ldots \ldots$. . . . . 91

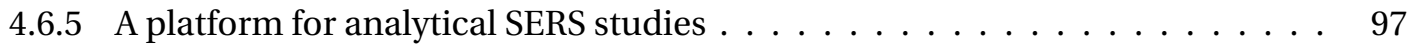

4.7 Conclusion and outlook $\ldots \ldots \ldots \ldots \ldots \ldots$

5 Overall conclusion $\quad 100$ 


\section{Chapter 1}

\section{Introduction}

Since Fleischmann, Hendra and McQuillan discovered an anomalously large signal of pyridine adsorbed on a silver (Ag) electrode in 1974 [3], surface enhanced Raman spectroscopy (SERS) has come a long way and grown into a large research field with entire books dedicated toward it $[2,4,5]$. While it was fairly soon understood by Jeanmaire and Van Duyne [6] and Moskovits [7] that the observed enhancement of the pyridine signal was of electromagnetic nature, there were other issues to which only recently satisfactory answers were found. Certainly one of the most prominent of them was centered around the question whether SERS allows the detection of single molecules and if so what the magnitude of the single molecule enhancement factor (SMEF) is (see [8-12] for further details).

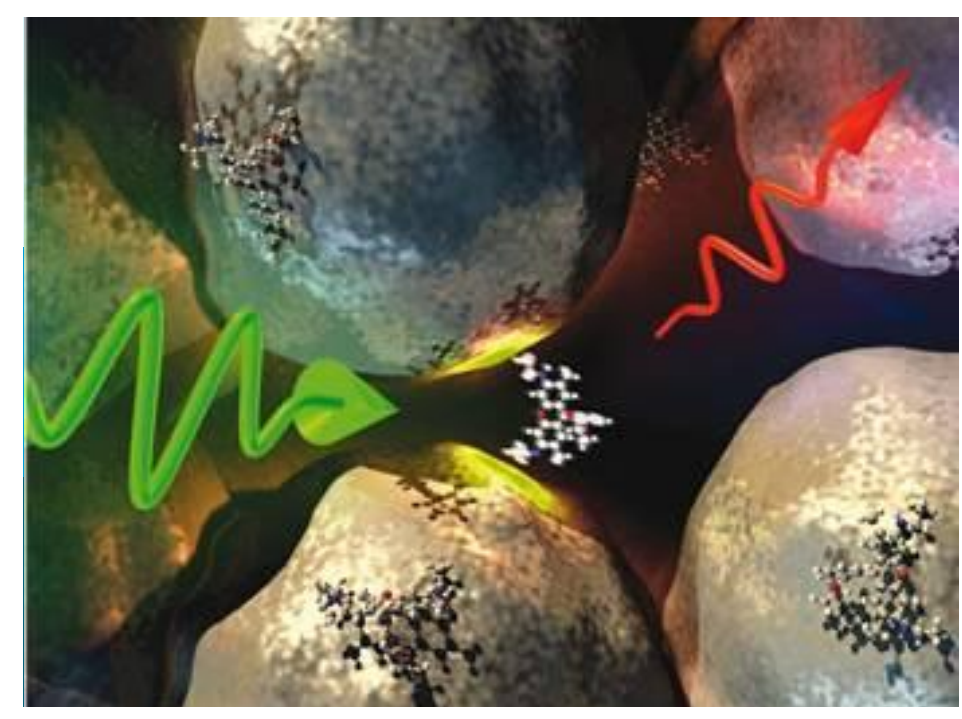

Fig. 1 : Artistic illustration of the SERS process with $\mathrm{Ag}$ nano-particles (friendly permission from M. Meyer).

On a practical level SERS allows one to overcome the major disadvantage of the Raman effect: its weakness in signal. Unfortunately this gain is bought at the expense of reproducibility giving rise to Natan's assessment in a review article [1] that SERS is governed by an "uncertainty principle": either the SERS-substrate's enhancement factor (EF) is good or its reproducibility - but not both. 
This is a rather unfortunate situation as it makes analytical work with quantitative measurements rather difficult. In Chapter 2 we would like to show a strategy how this deadlock can be broken and demonstrate its usefulness by measuring for the first time the resonant Raman cross-sections of Rhodamine 6G (RH6G) for seven excitation wavelengths across the visible range.

On the theoretical side it seems rather surprising that since Moskovits outlined in detail the surface selection rules (SSR) in SERS some 30 years ago [13], so far no one has ever confirmed them experimentally on a flat surface. This may be in part owed to the fact that it is challenging to find suitable experimental conditions with sufficient signal of a probe-molecule. Those conditions and the obtained results are outlined in Chapter $\mathbf{3}$ and are the most direct demonstrations of Moskovit's predictions published so far ${ }^{1}$.

In the final Chapter 4 the Kretschmann configuration will be discussed at length. Even though this setup is based on arguably the simplest plasmonic system available (namely a flat metallic film), it has received surprisingly little attention over the last few years. In our work we were able to show that with a clever and simple experimental configuration, two complementary techniques (namely SERS and surface-plasmon-resonance spectroscopy (SPRS)) can be combined and the signal efficiently collected. Especially because this latter result was achieved with minor modifications to a standard Raman-microscopy setup (unlike rather cumbersome prism setups described in previous publications), we hope that our results make SERS-microscopy on flat substrates an easily accessible tool.

As for the introduction given in this chapter, we aim at introducing only the most relevant terms and equations needed for understanding this thesis. For a more complete and in depth discussion of Raman spectroscopy and SERS we refer the interested reader to the excellent books available $[2,4,5,14]$.

\footnotetext{
${ }^{1}$ Given the fact that the modification of the Raman signal for a flat metallic surface illuminated from air is not due to a plasmonic effect and enhancement factors are mostly smaller than 1, one may argue that the work of this chapter should, strictly speaking, not be referred to in the context of SERS. But since the focus is on the verification of SSR for SERS a change in terminology (to Surface modified Raman Scattering) would only add confusion to a already challenging subject. Hence it shall only be emphasized at this point that the experimental situation we focus on in this chapter does not involve surface plasmon excitation and that furthermore the local field intensities are mostly quenched.
} 


\title{
1.1 Optical processes: a brief introduction
}

\begin{abstract}
Absorption is probably the most intuitive of all the processes that occur when light interacts with matter. I remember as a child running over the hot pavement on my way to the swimming pool in the summer because it had heated up to an unbearable temperature that would burn my feet. I was fascinated by the difference in temperature I would notice on an outdoor chess-board when going from a black square to a white one. Without knowing, I was experiencing the effect of absorption in which the photon energy of the incoming sunlight was transferred to the object exposed to it and eventually transformed into phonon-energy (i.e. heat). Naturally this transferprocess was more efficient for black objects than for white ones.
\end{abstract}

For our experiments we are interested in the absorption that happens with molecules in a solvent (like water or alcohol). Measuring the effect of absorption in a solution of given concentration by monitoring the change in temperature induced by exposing it to a well controlled light source with incoming power $P_{\text {inc }}$ would certainly be possible, but it would be rather crude. A better way to quantify the effect of absorption is by measuring the power $P$ of a monochromatic light beam of wavelength $\lambda$ before $\left(P_{\text {inc }}\right)$ and after $\left(P_{\text {tra }}\right)$ it passes through a length $L$ of solution. The ratio $A:=\log _{10}\left(P_{\text {inc }} / P_{\text {tra }}\right)$ is called absorbance and plotted in Fig. 2a) as a function of wavelength for a $1 \mu \mathrm{M}$ solution of RH6G in water and shows a clear peak around $525 \mathrm{~nm}$.
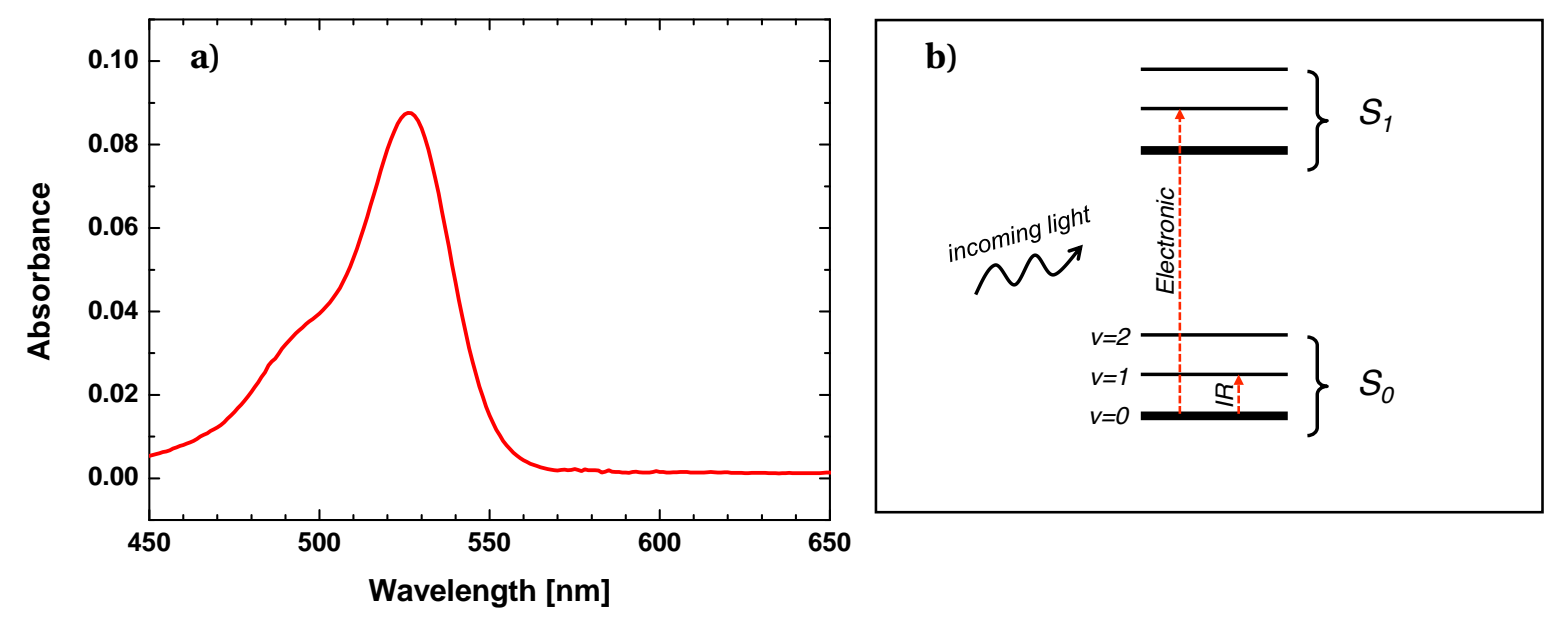

Fig. 2 : Two pictures illustrating absorption: a) absorbance A as a function of wavelength $\lambda$ for a $1 \mu M$ solution of RH6G (pathlength $1 \mathrm{~cm}$ ); b) Jablonski diagram of the absorption process in a molecule.

In order to get a better understanding of the absorption process on a quantum mechanical level, it is instructive to have a look at the Jablonski diagram depicted in Fig. 2b) where two different absorption processes are illustrated:

- Electronic absorption is a process in which the incoming photon has enough energy to excite the molecule into a higher electronic state. The typical energy needed is on the order of $3 \mathrm{eV}$ (a bit more for small molecules, a bit less for dyes). Light of that energy is in the visible or ultra-violet range resulting in the name UV/Vis spectroscopy. Because of its origin, this kind of measurement can serve as a probe for the electronic structure of a molecule. 
- When the incoming photon has only enough energy to excite the molecule into a higher vibrational level within the same electronic state (usually the ground state $S_{0}$ ) one talks about Infrared (IR) absorption. The energies involved are typically smaller than $0.5 \mathrm{eV}$ and correspond to a wavelength range between $3 \mu \mathrm{m}$ and $100 \mu \mathrm{m}$.

Fluorescence is the most common type of luminescence, the latter describing a class of processes characterized by absorption and consecutive emission of a photon by a molecule. It is a two step process illustrated in Fig. 3b) which can be described as follows:

- an incoming photon excites the molecule from its ground state $S_{0}$ to the first excited state $S_{1}$ (the previously discussed absorption). Via non-radiative transitions it relaxes to the lowest vibrational state in $S_{1}$ within a typical timescale of about 0.1 to $10 \mathrm{ps}$.

- having arrived at the lowest vibrational state, the molecule usually has a lifetime between 1 and $100 \mathrm{~ns}$ before spontaneous emission occurs entailing the emission of a photon (the observed fluorescence) and leaving it in any one of the vibrational states of $S_{0}$.

As can be appreciated from the above description, part of the absorbed photon's energy is lost in this cycle due to the non radiative emission processes that allow the molecule to relax to the lowest vibrational level in $S_{1}$. In consequence one can observe a clear red-shift from the emission to the absorption spectrum which is shown in Fig. 3a).
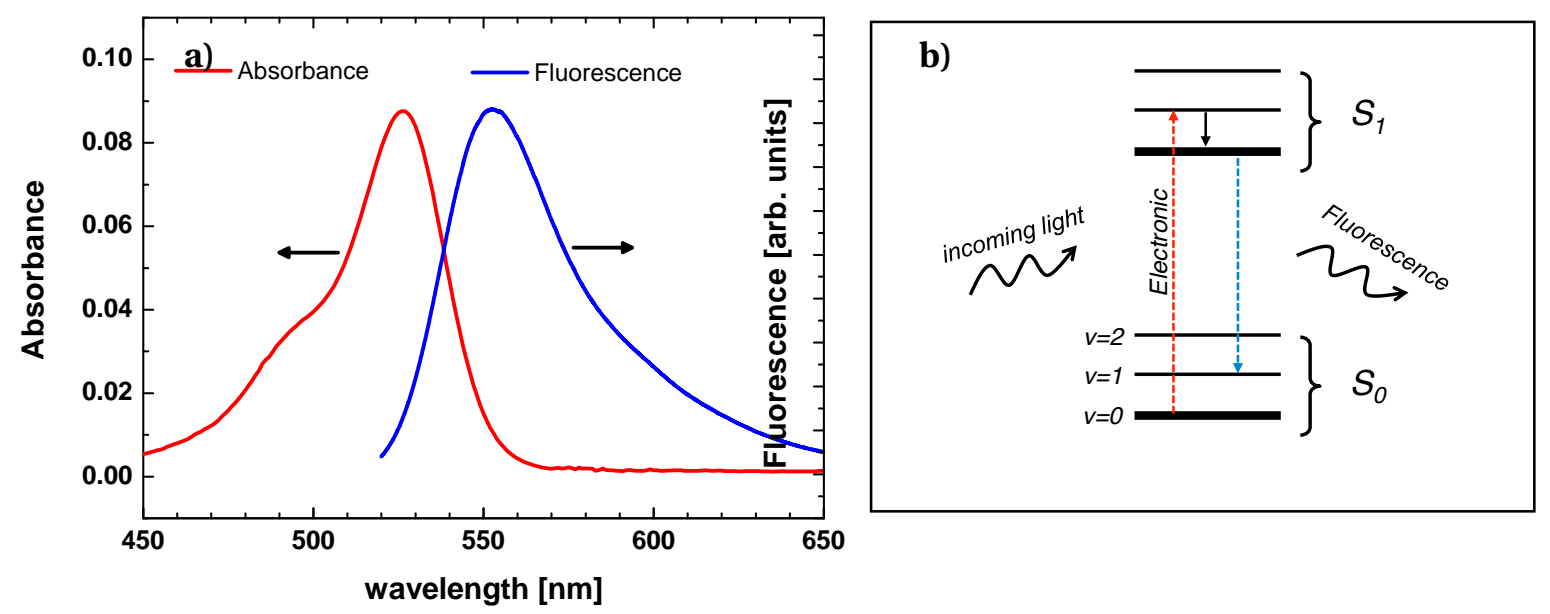

Fig. 3 : Two pictures illustrating fluorescence: a) fluorescence of a $400 \mathrm{nM}$ solution of RH6G in water as a function of wavelength with excitation at $514 \mathrm{~nm}$; b) Jablonski diagram illustrating the 2-step fluorescence process in a molecule.

It is worth mentioning at this point that fluorescence can easily be observed with bare eyes: when shining a green laser through a container with Nile-Blue solution one can easily see the laser beam appearing in red illustrating the effect of fluorescence.

Rayleigh scattering is an optical effect that we observe every day looking at the blue colour of the sky (Fig.4a)). Light from the sun is instantaneously scattered at the same wavelength by very small, gaseous molecules in the atmosphere. This process is more generally referred to as elastic 
light scattering and schematically illustrated in Fig. 4b). It is important to point out that for light scattering the energy of the absorbed photon does not have to match the energy of an electronic transition: a photon gets scattered by lifting the molecule to a virtual state. Through instantaneous relaxation back into the ground state a photon of equal energy but not necessarily equal direction or polarization is re-emitted.

As will be shown later on, the efficiency of this elastic scattering process is dependent on the frequency $\omega$ of the incident light and proportional to $\omega^{4}$. This means that shorter wavelengths (blue) are scattered much more efficiently than longer ones (red) leading to the already mentioned blue colour of the sky. In Raman spectroscopy, where a sample is excited by a laser, this Rayleighscattered light is unwanted (as it contains little information about the sample) and filtered in the detection beam path.
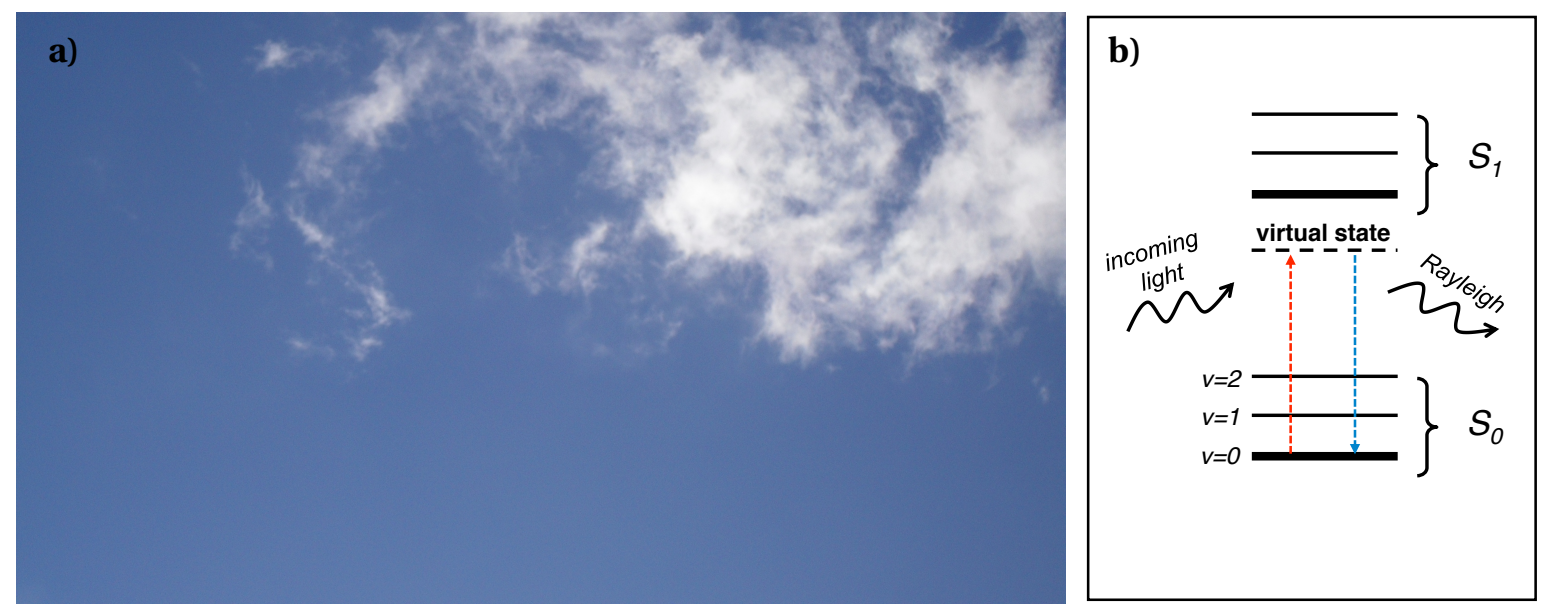

Fig. 4 : Two pictures illustrating Rayleigh scattering: a) sunlight is scattered by small atmospheric molecules more efficiently in the blue than in the red resulting in the blue colour the sky; $b$ ) Jablonski diagram illustrating the Rayleigh process.

The Raman effect is an inelastic scattering process for which incoming and scattered light differ in energy. This energy difference $\Delta E$ is caused by an instantaneous transition of the molecule between its vibrational states. If $\Delta E$ is positive (Stokes scattering) part of the incoming photon's energy was used to excite a vibration, if $\Delta E$ is negative (Anti-Stokes scattering) the vibration's energy was given to the scattered photon. It can be helpful to visualize this process as absorption and re-emission via an intermediate, virtual state as illustrated in Fig. 5 at the top.

Raman scattering was predicted by Smekal in 1923 [15] and originally discovered by C. V. Raman [16] on organic liquids by using sunlight for excitation and observing the Raman-shift with simple colour-filters and bare eyes. Even though it is good to know that the effect is indeed detectable so easily, more information about the molecule's vibrational energy levels can be extracted when looking at the scattered light with a more sophisticated setup.

Fig. 9 and 10 show the optical layout of the spectrometer used in most of our experiments where a laser serves as an excitation light source for a sample under observation. The double subtractive premonochromator stage blocks the elastically scattered light and makes observation of the Raman shift and the recording of a spectrum possible. 
The graph plotted in Fig. 5 shows such a spectrum in which the intensity of the scattered light is plotted in arbitrary units as a function of the energy shift. While the peak in the center at $0 \mathrm{~cm}^{-1}$ is (unwanted) stray-light of the laser, all the other peaks correspond to molecular vibrations. The symmetry of Stokes and anti-Stokes peak positions with respect to 0 is quite obvious. The reason that Stokes peaks are stronger in intensity is related to the population density of the molecule in the respective initial state: thermodynamically it is simply more likely to find the molecule in its ground state than in a given vibrational state and therefore the probability of an incoming photon to interact with the ground state is higher.

It is important to emphasize the difference between the Raman effect and the previously explained fluorescence: since the virtual state can, but does not have to, correspond to a real electronic state, the Raman effect is present even for incident light that does not have sufficient energy to excite the molecule into a higher electronic state and for which it would otherwise be transparent. Furthermore the instantaneous nature of the Raman effect has far reaching consequences for the observed enhancement when the molecule is placed in close proximity to a metal surface and differs significantly from the enhancement measured for fluorescence in the same environment. This is explained in more detail in Section 1.3.

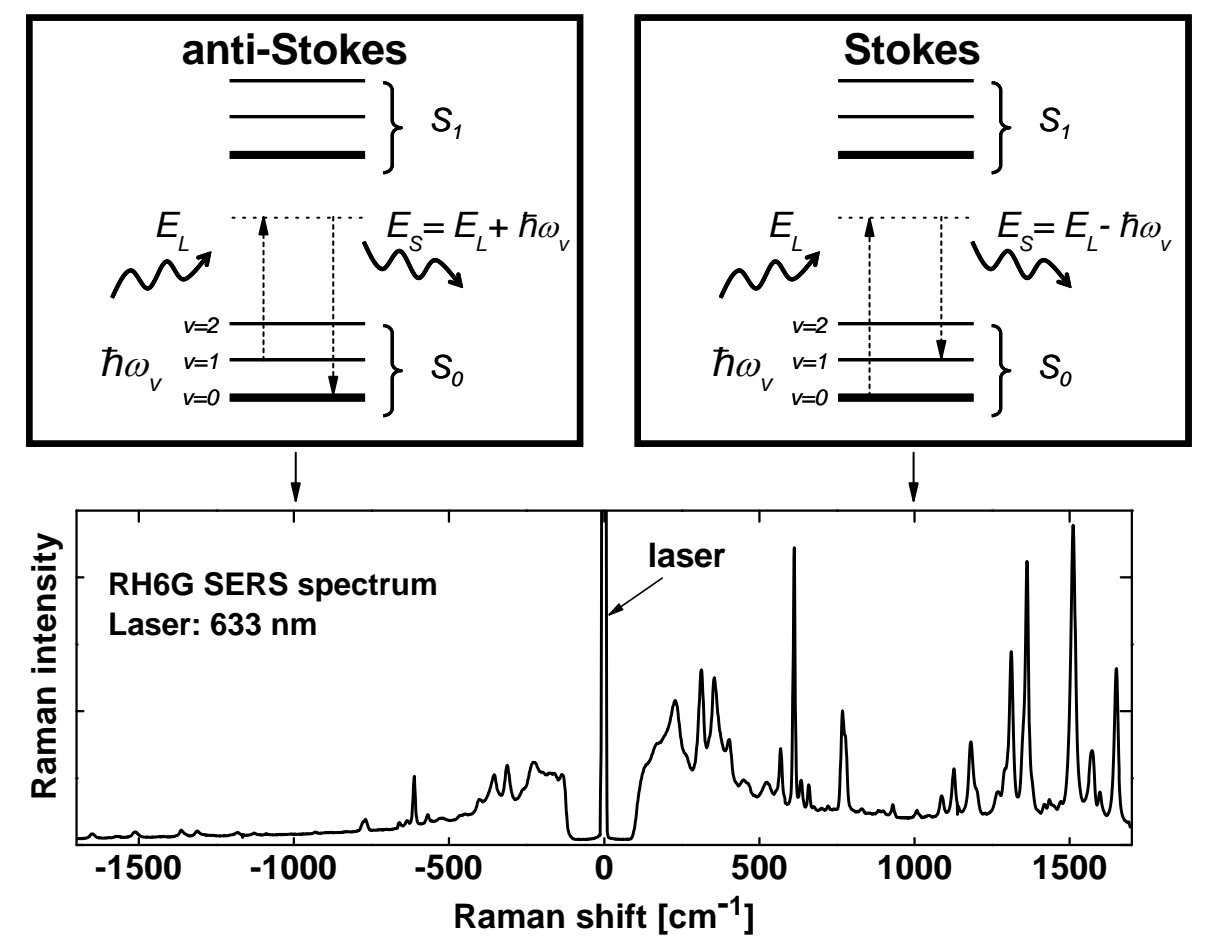

Fig. 5 : Three pictures illustrating Raman scattering: top) Jablonski diagram for the Stokes and antiStokes process in Raman scattering; bottom) Raman spectrum of RH6G as a function of wavenumber. The right hand side corresponds to Stokes-, the left hand side to anti-Stokes scattering; (from page 45 of [2]). 


\subsection{Optical processes: a quantitative description}

In the last Section the optical processes most relevant for this thesis (absorption, fluorescence, Rayleigh scattering and Raman scattering) were introduced in a rather qualitative way. In this section a more quantitative description shall be given. In order to do so, the concept of a scattering cross-section will be introduced and its analytical expression derived for each of the processes.

While for most of the discussion a classical approach is sufficient, the phenomenon of Resonant Raman Scattering can only be explained with a quantum mechanical approach. Since a complete treatment of this topic would be beyond the scope of this introduction, the end-result shall be given and its consequences explained but without the derivation. For further details see Long's book [14].

\subsubsection{The scattering cross-section}

The concept of a scattering cross-section is very general, used not only in optics but also e.g. in nuclear-physics. It is applied for any kind of situation in which a stream of incoming particles $\alpha$ (such as photons in optics or e.g. protons in Nuclear-Physics) interacts with a target. In such a situation one is usually interested in the probability to observe a certain interaction product $\beta$ at a given direction $\Omega=(\theta, \phi)$ for a certain flux $n\left[\frac{1}{\mathrm{sec} \cdot \mathrm{m}^{2}}\right]$ of incoming particles. This probability is called differential scattering cross-section $\frac{\mathrm{d} \sigma^{\alpha \rightarrow \beta}}{\mathrm{d} \Omega}(\Omega)\left[\frac{\mathrm{m}^{2}}{\mathrm{sr}^{2}}\right]$ and it connects the differential number of events $\frac{\mathrm{d} N^{\beta}}{\mathrm{d} \Omega}(\Omega)\left[\frac{1}{\mathrm{sec} \cdot \mathrm{sr}}\right]$ at a given angle $\Omega$ and for a given process $\alpha \rightarrow \beta$ with $n$ linearly:

$$
\frac{\mathrm{d} N^{\beta}}{\mathrm{d} \Omega}(\Omega)=\frac{\mathrm{d} \sigma^{\alpha \rightarrow \beta}}{\mathrm{d} \Omega}(\Omega) \cdot n^{\alpha}
$$

It is important to emphasize that $\frac{\mathrm{d} \sigma}{\mathrm{d} \Omega}$ (commonly in units of $\frac{\mathrm{cm}^{2}}{\mathrm{sr}}$ for optical spectroscopy) is a statistical term which only makes sense to be used in the context of a large number of events.

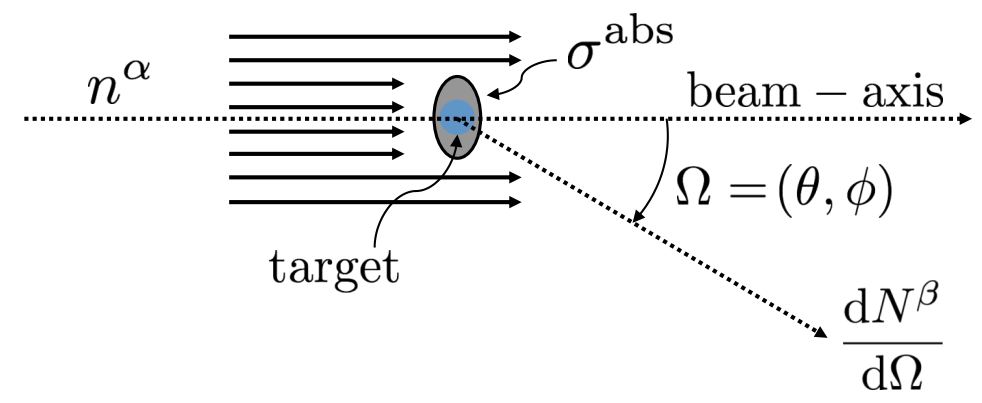

Fig. 6 : Picture illustrating the variables necessary to define the differential scattering cross-section.

In the case of optical absorption, it is fairly easy to attribute a physical meaning to the integrated or total differential cross-section $\sigma^{\text {abs }}$ which is implicitly defined by:

$$
P^{\mathrm{abs}}=: \sigma^{\mathrm{abs}} \cdot p^{\mathrm{inc}}
$$

If a molecule (the target) is exposed to an incoming, monochromatic beam of frequency $f\left[\frac{1}{\sec }\right]$ and power density $p^{\text {inc }}=n^{\text {inc }} \cdot h \cdot f\left[\frac{\mathrm{W}}{\mathrm{m}^{2}}\right]$ (where $h \cdot f[\mathrm{~J}]$ is the photon energy) we are interested 
the total absorbed power $P^{\text {abs }}[\mathrm{W}]$. Looking at eq. 1.2 it is apparent that the total scattering crosssection $\sigma^{\text {abs }}$ corresponds accordingly to the effective area $A_{\text {eff }}$ of that molecule: the area a perfectly black surface would have to have in order to absorb the exact same power as the molecule if placed in the beam.

In order to understand the physical meaning of the differential scattering cross-section better, let us consider the following situation: a monochromatic beam of power-density $p^{\text {inc }}$ impinges on a molecule with fixed orientation. This molecule in turn emits radiation (such as fluorescence, Rayleigh, Raman etc.) which can be observed with a detector in a given direction $\Omega$. Depending on the area of this detector and its distance $R$ from the molecule, it captures events in a certain solid angle $\Delta \Omega$ which is canonically given by the corresponding area $A$ on the unit sphere:

$$
\Delta \Omega=\frac{A}{R^{2}}
$$

Knowing the scattering cross-section $\frac{\mathrm{d} \sigma}{\mathrm{d} \Omega}$ for any of the mentioned processes allows us now to predict the scattered power $P^{\text {sca }}$ in a given direction $\Omega$ :

$$
P^{\text {sca }}(\Omega)=\frac{\mathrm{d} \sigma^{\text {sca }}}{\mathrm{d} \Omega}(\Omega) \cdot p^{\text {inc }} \cdot \Delta \Omega
$$

In the case of Raman spectroscopy, a molecule's scattering cross-section $\mathrm{d}_{\Omega} \sigma_{\mathrm{R}}$ depends not only on its orientation $\mathbf{t}$ and the detection angle $\Omega$, but also on incoming polarization $\mathbf{P}_{\mathrm{L}}$, detection polarization $\mathbf{P}_{\mathrm{R}}$, excitation wavelength $\lambda_{\mathrm{L}}$ and the refractive index $n=\sqrt{\epsilon}$ of the scattering environment which is assumed to be transparent. Since in a common Raman experiment ...

- ... the incoming light is usually linearly polarized and travels e.g. along $-\mathbf{e}_{z}$,

- ... the spectrometer is in a BS configuration meaning that $\phi=0^{\circ}$,

- ... the probe-molecule is in a random orientation, ...

... the definition of the differential Raman cross-section $\mathrm{d}_{\Omega} \sigma_{\text {rad }}^{\mathrm{R}}$ is slightly more specific than eq. 1.1 and implicitly defined as:

$$
\frac{\mathrm{d} P_{\mathrm{R}}}{\mathrm{d} \Omega}\left(\phi=0^{\circ}, \mathbf{t}=\text { rand., } \mathbf{P}_{\mathrm{L}}=\mathbf{e}_{x}\right)=: \frac{\mathrm{d} \sigma_{\mathrm{rad}}^{\mathrm{R}}}{\mathrm{d} \Omega} \cdot p^{\text {inc }}
$$

If in addition to the incoming polarization $\mathbf{P}_{\mathrm{L}}$, the outgoing polarization $\mathbf{P}_{\mathrm{R}}$ is defined as well, then the perpendicular $(\perp)$ and parallel $(\|)$ scattering cross-sections can be defined along with the depolarization ration $\rho_{\mathrm{R}}$ as follows:

$$
\left.\begin{array}{l}
\mathrm{d}_{\Omega} \sigma_{\text {rad }}^{\mathrm{R}-\perp} \text { for } \mathbf{P}_{\mathrm{R}} \perp \mathbf{P}_{\mathrm{L}} \\
\mathrm{d}_{\Omega} \sigma_{\text {rad }}^{\mathrm{R}-\|} \text { for } \mathbf{P}_{\mathrm{R}}=\mathbf{P}_{\mathrm{L}}
\end{array}\right\} \text { and } \rho_{\mathrm{R}}:=\frac{\mathrm{d}_{\Omega} \sigma_{\text {rad }}^{\mathrm{R}-\perp}}{\mathrm{d}_{\Omega} \sigma_{\text {rad }}^{\mathrm{R}-\|}}
$$

In order to get a better idea about the numbers involved, the following table shows typical values for $\frac{\mathrm{d} \sigma}{\mathrm{d} \Omega}$-values encountered for fluorescence, Raman and resonant Raman scattering for the example of RH6G. 


\begin{tabular}{lc} 
process & $\frac{\mathrm{d} \sigma}{\mathrm{d} \Omega}$ \\
\hline absorption & $\sim 10^{-16} \mathrm{~cm}^{2}$ \\
fluorescence & $\sim 10^{-17 \frac{\mathrm{cm}^{2}}{\mathrm{sr}}}$ \\
non-resonant Raman & $\sim 10^{-31}-10^{-27} \frac{\mathrm{cm}^{2}}{\mathrm{sr}}$ \\
resonant Raman & $\sim 10^{-24} \frac{\mathrm{cm}^{2}}{\mathrm{sr}}$ \\
\hline
\end{tabular}

It is important to point out that while both - fluorescence and Raman cross-sections - refer to intensities that are integrated over a certain spectral range, Raman peaks are much narrower in spectral width and should therefore be multiplied with a factor of $10^{2}-10^{3}$ to be able to make a fair comparison.

\subsubsection{Absorption}

For many of the experiments presented in this thesis, a good understanding of the absorption behavior (and therefore of the absorption cross-section $\sigma^{\text {abs }}$ ) of the probe-molecule is necessary. A common way to obtain this information is with a UV/Vis spectrometer: the molecule of interest (with absorption cross-section $\sigma^{\text {abs}}$ ) is dissolved in a transparent liquid at a concentration $c$ [mol] in a container of length $\Delta L$. This specimen is then illuminated by a monochromatic beam of area $A$ and power-density $p^{\text {inc }}$ allowing us to learn about the sample by measuring the transmitted power $P^{\text {tra }}$ on the other side.

In order to derive $P^{\text {tra }}$ as a function of these input parameters, one can start by using conservation of energy to write the incoming power $P(x)$ as a sum of transmitted, absorbed and scattered power for a small volume element $\Delta V:=A \Delta \cdot L$ :

$$
P(x)=P(x+\Delta L)+P_{\Delta V}^{\text {abs }}+P_{\Delta V}^{\text {sca }} \stackrel{\text { eq.1.2 }}{=} P(x+\Delta L)+N^{\Delta V} \cdot\left(\sigma^{\text {abs }}+\sigma^{\text {sca }}\right) \cdot p(x)
$$

Here $N^{\Delta V}=N_{\mathrm{Av}} \cdot c \cdot \Delta V$ is the number of molecules in $\Delta V$ and the combined effect of absorption and scattering is summarized in the extinction cross-section $\sigma^{\text {ext }} \equiv \sigma^{\text {abs }}+\sigma^{\text {sca }}$. This renders immediately:

$$
\begin{aligned}
& \overbrace{P(x+\Delta L)-P(x)}^{\Delta P}=-\overbrace{N_{\mathrm{Av}} \cdot c \cdot \Delta L \cdot A}^{N^{\Delta V}} \cdot \overbrace{\left(\sigma^{\text {abs }}+\sigma^{\text {sca }}\right)}^{\sigma^{\text {ext }}} \cdot p(x) \\
& \stackrel{A \cdot p \equiv P}{\Longrightarrow} \frac{\Delta P}{\Delta L}(x)=-N_{\mathrm{Av}} c \sigma^{\mathrm{ext}} \cdot P(x)
\end{aligned}
$$

The last equation can be interpreted as a differential equation with the simple Beer-Lambert Law as its solution:

$$
P(x)=P_{0} \cdot \exp \left(-N_{\mathrm{Av}} c \sigma^{\mathrm{ext}} \cdot x\right)
$$

This means that the transmittance $T:=P^{\text {tra }} / P^{\text {inc }}=P(x) / P_{0}$ can be written as $T=\exp \left(-N_{\mathrm{Av}} c \sigma^{\text {ext }} \cdot L\right)$, which allows us (with known concentration $c$ and container length $\Delta L$ ) to deduce the extinction cross-section $\sigma^{\text {ext }}$. Since for most molecules $\sigma^{\text {sca }} \ll \sigma^{\text {abs }}$, it is reasonable to approximate 
$\sigma^{\mathrm{ext}} \approx \sigma^{\text {abs }}$ which then renders us with the desired quantity. It is important to point out that most UV-Vis spectrometers' output is not $T$ but the absorbance $A:=-\log _{10}(T)=\frac{N_{A v} c \sigma^{\text {ext }} \Delta L}{\ln (10)}$ and hence:

$$
\sigma^{\mathrm{abs}} \approx \sigma^{\mathrm{ext}}=A \cdot \frac{\ln (10)}{N_{\mathrm{Av}} c \cdot \Delta L}
$$

\subsubsection{Fluorescence}

As has been explained already, fluorescence is a two step process consisting of the excitation of a molecule into an electronic state $S_{1}$ through absorption of an incoming photon followed by the spontaneous emission of a photon concomitant with the molecule's relaxation back into $S_{0}$. Just like absorption, fluorescence has a total cross-section $\sigma^{\text {fluo }}$ that allows quantitative comparison with the other types of radiation and shall be derived in the following.

In order to do so, let us look at the second step of the fluorescence process which is characterized by the radiative decay rate $\Gamma^{\mathrm{rad}}\left[\frac{1}{\mathrm{sec}}\right]$ (commonly on the order of $10^{9} / \mathrm{sec}$ ). Even though the relaxation from $S_{1}$ into $S_{0}$ can be associated with the spontaneous emission of a photon, it is by no means imperative and can just as well happen through a non-radiative process which is in turn characterized by $\Gamma^{\mathrm{NR}}$. The sum of these decay rates constitutes the total decay rate $\Gamma^{\text {tot }}:=\Gamma^{\mathrm{rad}}+\Gamma^{\mathrm{NR}}$ which allows us a straight forward definition of the fluorescence quantum yield:

$$
Q:=\frac{\Gamma^{\mathrm{rad}}}{\Gamma^{\mathrm{tot}}}
$$

This number describes the competition between radiative and non-radiative decay and is close to 1 for many dye molecules (meaning that almost all of the absorbed energy is re-emitted as fluorescence). The integrated power of this fluorescence radiation is therefore:

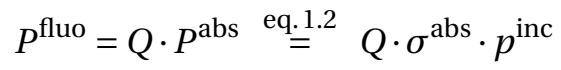

By definition of the total fluorescence cross-section, we can therefore conclude that:

$$
\sigma^{\text {Fluo }}=Q \cdot \sigma^{\text {abs }}
$$

Since for many dye molecules $Q \approx 1$, knowledge of the absorption cross-section renders also $\sigma^{\text {fluo }}$. For other molecules $\sigma^{\text {fluo }}$ has to be inferred from measuring its absorption and subsequently comparing its fluorescence spectrum to that of a molecule with known absorption and quantum yield.

\subsubsection{Rayleigh scattering}

In Section 1.1 the blue colour of the sky was given as an example for Rayleigh radiation, which occurs when light is scattered on objects (e.g. air molecules) that are much smaller than the incident wavelength. While the previous explanation was more qualitative, the objective now is to come up with a quantitative description that allows us to write down the Rayleigh scattering cross-section 
$\sigma^{\mathrm{RL}}$ explicitly. Since the latter is implicitly defined by the response of a small object to some outside excitation, we first have to derive the power $P^{\mathrm{RL}}$ scattered by such an object when exposed to mono-chromatic light of power-density $p^{\text {inc }}$.

In order to do so, let us consider a nitrogen molecule in the atmosphere that is struck by radiation of a certain angular frequency $\omega$ (e.g. part of the sun's radiation). This can be modeled as inducing a dipole $\mathbf{p}(\omega)$ that oscillates at the same frequency $\omega$ according to:

$$
\mathbf{p}(\omega)=\hat{\alpha}_{\mathrm{RL}}(\omega) \cdot \mathbf{E}(\omega)
$$

Here $\hat{\alpha}_{\mathrm{RL}}$ is the linear or Rayleigh polarizability tensor. By treating the interaction of the electromagnetic wave with an electronic state of the Nitrogen atom as a classical harmonic oscillator with eigen-frequency $\omega_{0}\left[\frac{\mathrm{rad}}{\mathrm{sec}}\right]$ one can derive the scalar function $\alpha_{\mathrm{RL}}(\omega)$. This is done in the Lorentz model and it yields the expression (see [2], appendix D):

$$
\alpha_{\mathrm{RL}}(\omega)=\frac{e^{2} / m}{\omega_{0}^{2}-\omega^{2}-i \gamma \omega}
$$

Here $e$ and $m$ are the effective charge and mass of the electronic cloud (respectively) and $\gamma$ is a dissipation coefficient accounting for internal and external losses. With this expression it remains now to understand the emission profile of a dipole $\mathbf{p}$ oscillating at an excitation frequency $\omega$.

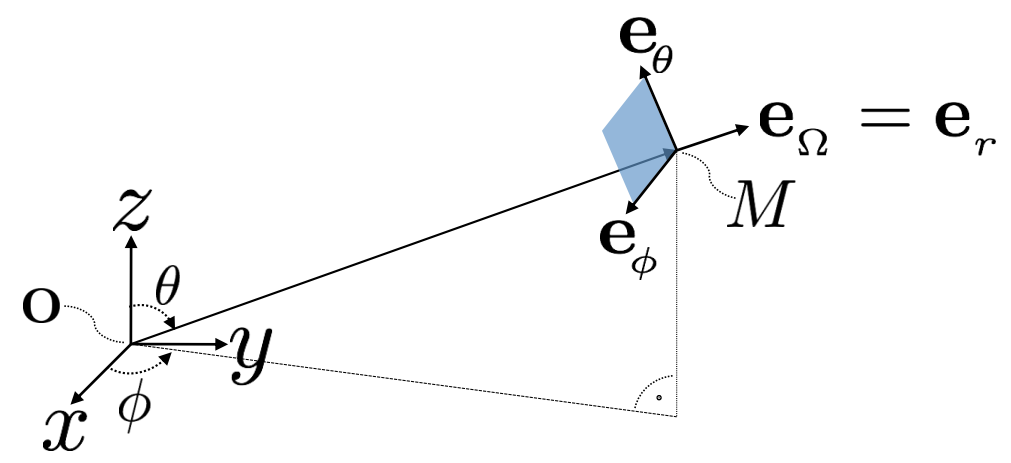

Fig. 7:

Schematic illustration of a spherical coordinate system, its variables and the corresponding unit-vectors.

For this purpose let us consider a free dipole $\mathbf{p} \equiv|\mathbf{p}| \cdot \mathbf{e}_{z}$ located at the origin $\mathrm{O}$ of the coordinate system depicted in Fig. 7 and a power-meter at angle $\Omega \equiv(\theta, \phi)$ at M with a polarizer oriented along $\mathbf{e}_{\mathrm{Pol}} \in \operatorname{span}\left(\mathbf{e}_{\theta}, \mathbf{e}_{\phi}\right)$ in front of it. It is a standard exercise demonstrated in many text books to calculate the differential power $\mathrm{d} P_{\mathrm{rad}}^{\mathrm{FD}-\mathrm{Pol}} / \mathrm{d} \Omega$ measured in $\mathrm{M}$. Therefore only the result shall be stated here (from page 73 of [2], eq. 2.36, only the factor $\sqrt{\epsilon_{M}}$ accounting for surrounding medium had to be added):

$$
\begin{aligned}
\frac{\mathrm{d} P_{\mathrm{rad}}^{\mathrm{FD}-\mathrm{Pol}}}{\mathrm{d} \Omega} & =C_{p} C_{E}^{2} \omega^{4}\left|\mathbf{p} \cdot \mathbf{e}_{\mathrm{Pol}}\right|^{2} \\
& =\underbrace{C_{p} C_{E}^{2}}_{1} \underbrace{\omega^{4}|\mathbf{p}|^{2}}_{2} \underbrace{\left|\mathbf{e}_{\mathrm{p}} \cdot \mathbf{e}_{\mathrm{Pol}}\right|^{2}}_{3}
\end{aligned}
$$

Here $\mathbf{e}_{\mathrm{p}}$ is just a shorthand notation for $\mathbf{p} /|\mathbf{p}|$ that allows us to use this equation more easily in the future for any dipole orientation. To better understand this expression let us analyze the terms 1 , 2 and 3 in more detail: 
- Term 1 is product of the constants $C_{p}:=\sqrt{\epsilon_{\mathrm{M}}} \epsilon_{0} c / 2$ and $C_{E}:=\frac{1}{4 \pi \epsilon_{0} c^{2}}$ which are just convenient substitutions stemming from the derivation and yielding:

$$
C_{p} \cdot C_{E}^{2}=\frac{\sqrt{\epsilon_{M}}}{32 \pi^{2} \epsilon_{0} c^{3}}
$$

- Term 2 is of great interest as it contains two non linear contributions: the $\omega^{4}$ factor is the underlying reason for the sky appearing to be blue since higher frequencies (i.e. blue) in the solar spectrum get scattered much more efficiently than lower frequencies (i.e. red). The factor $|\mathbf{p}|^{2}$ is of great interest as it allows us with eq. 1.15 and the Lorentz model to establish a connection between the radiated power $\mathrm{d} P_{\mathrm{rad}}^{\mathrm{FD}-\mathrm{Pol}} / \mathrm{d} \Omega$ and the incoming power density $p^{\text {inc }}=C_{p} \cdot|\mathbf{E}|^{2}$ (page 46, [2]) which is necessary to arrive at an expression for $\mathrm{d} \sigma / \mathrm{d} \Omega$ :

$$
|\mathbf{p}|^{2} \stackrel{\text { eq.1.15 }}{=}|\alpha|^{2} \cdot|\mathbf{E}|^{2}=\frac{|\alpha|^{2}}{C_{p}} \cdot p^{\text {inc }}
$$

- Term 3 gives us a clear understanding of the radiation profile. From Fig. 7 it is obvious that:

$$
\begin{aligned}
\mathbf{e}_{\mathrm{p}} \cdot \mathbf{e}_{\mathrm{Pol}} & =[\underbrace{\left(\mathbf{e}_{\mathrm{p}} \cdot \mathbf{e}_{r}\right) \mathbf{e}_{r}}_{\text {no contribution }}+\underbrace{\left(\mathbf{e}_{\mathrm{p}} \cdot \mathbf{e}_{\phi}\right)}_{=0} \mathbf{e}_{\phi}+\underbrace{\left(\mathbf{e}_{\mathrm{p}} \cdot \mathbf{e}_{\theta}\right)}_{\sin \theta} \mathbf{e}_{\theta}] \cdot \mathbf{e}_{\mathrm{Pol}} \\
\Rightarrow\left|\mathbf{e}_{\mathrm{p}} \cdot \mathbf{e}_{\mathrm{Pol}}\right|^{2} & =\sin ^{2}(\theta) \cdot \cos ^{2}\left(\mathbf{e}_{\mathrm{Pol}}, \mathbf{e}_{\theta}\right)
\end{aligned}
$$

This means that the best output is achieved in the horizontal direction $\left(\theta=90^{\circ}\right)$ and for the polarizer aligned with $\mathbf{e}_{\theta}$. By either looking along the dipolar axis $\left(\theta=0^{\circ}\right)$ or turning the polarizer perpendicular to $\mathbf{e}_{\theta}$ the radiation vanishes.

Inserting eq. 1.20 into eq. 1.18 renders an expression that brings us very close to the desired Rayleigh scattering cross-section:

$$
\frac{\mathrm{d} P_{\mathrm{rd}}^{\mathrm{FD}-\text { Pol }}}{\mathrm{d} \Omega}=\underbrace{C_{E}^{2} \omega^{4}|\alpha|^{2}\left|\mathbf{e}_{\mathrm{p}} \cdot \mathbf{e}_{\mathrm{Pol}}\right|^{2}}_{\mathrm{d} \sigma^{\mathrm{RL} / \mathrm{d} \Omega}} \cdot p^{\mathrm{inc}}
$$

A free dipole $\mathbf{p} \equiv|\mathbf{p}| \cdot \mathbf{e}_{\mathrm{p}}$ has hence a Rayleigh scattering cross-section of:

$$
\frac{\mathrm{d} \sigma^{\mathrm{RL}}}{\mathrm{d} \Omega}=\frac{\omega^{4}|\alpha|^{2}}{16 \pi^{2} \epsilon_{0}^{2} c^{4}}\left|\mathbf{e}_{\mathrm{p}} \cdot \mathbf{e}_{\mathrm{Pol}}\right|^{2}
$$

\subsubsection{Raman scattering}

In the previous subsection we derived a molecule's Rayleigh cross-section with an isotropic Raman tensor in vacuum. Instead of generalizing this expression for a non-isotropic molecule with a fixed position in a dielectric medium, we'll go in this subsection straight to the case of Raman scattering.

In order to derive the cross-section for the case of Raman emission at angular frequency $\omega_{\mathrm{R}}$, it is possible to generalize the phenomenological approach described in the previous paragraphs for Rayleigh scattering. By looking at inelastic scattering classically as dipole-radiation we can copy a lot of the things already done. There are, however, a number of subtle but important differences which need to be discussed in detail: 
- Firstly, the expression that connects the induced Raman dipole $\mathbf{p}_{\mathrm{R}}\left(\omega_{\mathrm{R}}\right)$ with the electric field $\mathbf{E}_{\mathrm{L}}\left(\omega_{\mathrm{L}}\right)$ of the laser (with angular frequency $\left.\omega_{\mathrm{L}}\right)$ needs to contain a local field correction factor $L_{\mathrm{m}}^{1 / 4}:=\left(\epsilon_{\mathrm{m}}+2\right) / 3$ to account for the microscopic effects that cause the molecule to "feel" a higher field than the macroscopic one governed and described by the Maxwell equations (see page 79, [2]):

$$
\mathbf{p}\left(\omega_{\mathrm{R}}\right)=L_{\mathrm{m}}^{1 / 4} \cdot \hat{\alpha}_{\mathrm{R}}\left(\omega_{\mathrm{L}}, \omega_{\mathrm{R}}\right) \cdot \mathbf{E}\left(\omega_{\mathrm{L}}\right)
$$

- Secondly, by virtue of reciprocity the local field correction impacts emission, too, and eq. 1.17 has to by corrected by a factor $\sqrt{L_{\mathrm{m}}}$ :

$$
\frac{\mathrm{d} P_{\mathrm{rad}}^{\mathrm{R}-\mathbf{P}_{\mathrm{R}}}}{\mathrm{d} \Omega}=\sqrt{L_{\mathrm{m}}} \cdot C_{p} C_{E}^{2} \cdot \omega^{4} \cdot\left|\mathbf{e}_{\mathbf{P}_{\mathrm{R}}} \cdot \mathbf{p}\right|^{2}
$$

Inserting eq. 1.25 into eq. 1.26 yields an equation that takes us already very close to the desired Raman cross-section (we use $p^{\text {inc }}=C_{p} \cdot|\mathbf{E}|^{2}$ and $C_{E}:=\frac{1}{4 \pi \epsilon_{0} c^{2}}$ going from the first line to the second):

$$
\begin{aligned}
\frac{\mathrm{d} P_{\mathrm{rad}}^{\mathrm{R}-\mathbf{P}_{\mathrm{R}}}}{\mathrm{d} \Omega} & =L_{\mathrm{m}} \cdot C_{p} C_{E}^{2} \cdot \quad \omega^{4} \cdot\left|\mathbf{e}_{\mathbf{P}_{\mathrm{R}}} \cdot \hat{\alpha}_{\mathrm{R}}\left(\omega_{\mathrm{L}}, \omega_{\mathrm{R}}\right) \cdot \mathbf{E}\left(\omega_{\mathrm{L}}\right)\right|^{2} \\
& =\underbrace{L_{\mathrm{m}} \frac{1}{16 \pi^{2} \epsilon_{0}^{2} c^{4}} \cdot \omega^{4} \cdot\left|\mathbf{e}_{\mathbf{P}_{\mathrm{R}}} \cdot \hat{\alpha}_{\mathrm{R}}\left(\omega_{\mathrm{L}}, \omega_{\mathrm{R}}\right) \cdot \mathbf{e}_{\mathrm{L}}\right|^{2}}_{\text {non-averaged } \mathrm{d}_{\Omega} \sigma} \cdot p^{\mathrm{inc}}
\end{aligned}
$$

From this equation we can already see how the cross-section for a molecule in a specific orientation would look like. However, since the definition of the Raman cross-section (as specified in eq. 1.5) specifically refers to a molecule in a random orientation, the orientation-average has to be taken which yields the following expression:

$$
\frac{\mathrm{d} \sigma_{\mathrm{rad}}^{\mathrm{R}-\mathbf{P}_{\mathrm{R}}}}{\mathrm{d} \Omega}=L_{\mathrm{m}} \frac{\omega^{4}}{16 \pi^{2} \epsilon_{0}^{2} c^{4}}\left\langle\left|\mathbf{e}_{\mathbf{P}_{\mathrm{R}}} \cdot \hat{\alpha}_{\mathrm{R}}\left(\omega_{\mathrm{L}}, \omega_{\mathrm{R}}\right) \cdot \mathbf{e}_{\mathrm{L}}\right|^{2}\right\rangle
$$

In order to write this more explicitly the orientation-average $\left\langle\left|\mathbf{e}_{\mathrm{Pol}} \cdot \hat{\alpha}_{\mathrm{R}}\left(\omega_{\mathrm{L}}, \omega_{\mathrm{R}}\right) \cdot \mathbf{e}_{\mathrm{L}}\right|^{2}\right\rangle$ has to be carried out. This shall not be done in detail here but the result shall be given for the two extreme cases with $\mathbf{e}_{\mathrm{Pol}} \| \mathbf{e}_{\mathrm{L}}$ and $\mathbf{e}_{\mathrm{Pol}} \perp \mathbf{e}_{\mathrm{L}}$ (from p. 84, [2]):

$$
\begin{array}{lll}
\left\langle\left|\mathbf{e}_{x} \cdot \hat{\alpha}_{\mathrm{R}}\left(\omega_{\mathrm{L}}, \omega_{\mathrm{R}}\right) \cdot \mathbf{e}_{x}\right|^{2}\right\rangle=\frac{45 \bar{\alpha}^{2}+4 \bar{\gamma}^{2}}{45} & \stackrel{\text { eq.1.28 }}{\Longrightarrow} \quad \frac{\mathrm{d} \sigma_{\mathrm{rad}}^{\mathrm{R}-\|}}{\mathrm{d} \Omega}=L_{\mathrm{m}} \frac{\omega^{4}}{16 \pi^{2} \epsilon_{0}^{2} c^{4}} \cdot \frac{45 \bar{\alpha}^{2}+4 \bar{\gamma}^{2}}{45} \\
\left\langle\left|\mathbf{e}_{x} \cdot \hat{\alpha}_{\mathrm{R}}\left(\omega_{\mathrm{L}}, \omega_{\mathrm{R}}\right) \cdot \mathbf{e}_{y}\right|^{2}\right\rangle=\frac{3 \bar{\gamma}^{2}}{45} & \stackrel{\text { eq. } 1.28}{\Longrightarrow} & \frac{\mathrm{d} \sigma_{\mathrm{rad}}^{\mathrm{R}-\perp}}{\mathrm{d} \Omega}=L_{\mathrm{m}} \frac{\omega^{4}}{16 \pi^{2} \epsilon_{0}^{2} c^{4}} \cdot \frac{3 \bar{\gamma}^{2}}{45}
\end{array}
$$

In this equation the reduced trace $\bar{\alpha}$ and the anisotropy parameter $\bar{\gamma}$ are two scalar invariants of the tensor which are defined as follows:

$$
\begin{aligned}
& \bar{\alpha}^{2} \equiv \frac{1}{9}\left(\alpha_{x x}+\alpha_{y y}+\alpha_{z z}\right)^{2} \\
& \bar{\gamma}^{2} \equiv \frac{1}{2}\left[\left(\alpha_{x x}-\alpha_{y y}\right)^{2}+\left(\alpha_{x x}-\alpha_{z z}\right)^{2}+\left(\alpha_{y y}-\alpha_{z z}\right)^{2}\right]+3\left[\alpha_{x y}^{2}+\alpha_{x z}^{2}+\alpha_{y z}^{2}\right]
\end{aligned}
$$


The total Raman cross-section is the sum of the perpendicular and parallel one and therefore:

$$
\frac{\mathrm{d} \sigma_{\mathrm{rad}}^{\mathrm{R}}}{\mathrm{d} \Omega}=L_{\mathrm{m}} C_{E}^{2} \omega^{4} \cdot|\hat{\alpha}|^{2}=L_{\mathrm{m}} \frac{\omega^{4}}{16 \pi^{2} \varepsilon_{0}^{2} c^{4}} \cdot|\hat{\alpha}|^{2}
$$

In this equation $|\hat{\alpha}|^{2}:=\left(45 \bar{\alpha}^{2}+7 \bar{\gamma}^{2}\right) / 45$ is the squared magnitude of the tensor $\hat{\alpha}$ (p.86, [2]). This invariant is very useful as it allows us to define the normalized Raman polarizability tensor, of which we will make extensive use in the next section:

$$
\hat{\alpha}_{\mathrm{N}}:=\frac{\hat{\alpha}}{|\hat{\alpha}|}
$$

While in general the calculation of $\hat{\alpha}$ is quite a significant challenge that requires e.g. density functional theory, for a uni-axial molecule oriented along $\mathbf{e}_{z}$ the tensor $\hat{\alpha}_{\mathrm{N}}$ has a very simple form ${ }^{2}$ :

$$
\hat{\alpha}_{\mathrm{N}}^{\mathbf{e}_{z}}=\sqrt{15 / 4}\left(\begin{array}{ccc}
0 & 0 & 0 \\
0 & 0 & 0 \\
0 & 0 & 1
\end{array}\right)
$$

\subsubsection{Resonant Raman scattering}

Resonant Raman scattering (RRS) occurs when the photon energy $E_{\mathrm{L}}$ of the exciting light source matches the energy of an electronic transition in the molecule. This situation is described by the Jablonski diagram depicted in Fig. 5 but with the transition's virtual state (the dotted line) matching a real electronic state of the molecule (e.g. S1).

The interesting thing about RRS is the fact that the associated cross-sections become disproportionately large, an effect that cannot be explained with the classical approach used so far. The semi-classical one however gives a good qualitative explanation for these high cross-sections. In it the electric field is still described classically, but the molecule is treated quantum mechanically and therefore its transition between initial state $|i\rangle$ and final state $|f\rangle$ characterized with a probability $\alpha_{k l}$ given by (p.100, [2]):

$$
\alpha_{k l}=\sum_{r \neq i, f}\left\{\frac{\left\langle f\left|P_{k}\right| r\right\rangle\left\langle r\left|P_{l}\right| i\right\rangle}{E_{r}-E_{i}-E_{\mathrm{L}}-i \hbar \Gamma_{r}}+\frac{\left\langle f\left|P_{l}\right| r\right\rangle\left\langle r\left|P_{k}\right| i\right\rangle}{E_{r}-E_{f}+E_{\mathrm{L}}+i \hbar \Gamma_{r}}\right\}
$$

Here the sum is over all possible states $|r\rangle$. Here the dipole moment operators $P_{k}$ and $P_{l}$ allow us to assign these probabilities to the components of the Raman tensor establishing the link between the classical description of the field and the quantum mechanical approach of the molecule.

It is obvious that if the energy difference between intermediate and initial state $E_{r}-E_{i}$ is close to the the incident photon energy $E_{\mathrm{L}}$ the denominator becomes very small resulting in a resonance.

\footnotetext{
${ }^{2}$ With the definition for the magnitude of a tensor given in the previous paragraph it is easy to see that $\left|\hat{\alpha}_{\mathrm{N}}^{\mathbf{e}_{z}}\right|^{2}=1$ and therefore properly normalized.
} 


\subsection{Enhanced Raman and fluorescence}

In the last section the differential Raman radiation $\mathrm{d} P_{\mathrm{rad}}^{\mathrm{R}} / \mathrm{d} \Omega$ of a randomly oriented molecule was derived which in turn allowed us to deduce the differential Raman cross-section $\mathrm{d}_{\Omega} \sigma_{\mathrm{rad}}^{\mathrm{R}}$ :

$$
\frac{\mathrm{d} P_{\mathrm{rad}}^{\mathrm{R}}}{\mathrm{d} \Omega}=\underbrace{L_{\mathrm{m}} C_{E}^{2} \omega^{4}|\hat{\alpha}|^{2}}_{\mathrm{d}_{\Omega} \sigma} \cdot p^{\mathrm{inc}}
$$

This is a very useful result as it corresponds to a very common experimental situation in which a large number of molecules (e.g. of a given dye) in a water solution are examined with a Raman spectrometer.

In this section we would like to look at a more complex situation in which a molecule on a metallic surface is illuminated with a laser. In this situation the molecule not only experiences a modified local field strength, but also its Raman signal is altered due to the presence of the metal. Our goal will be to derive the differential power $\mathrm{d} P_{\mathrm{rad}}^{\mathrm{SERS}-\mathbf{P}_{\mathrm{R}}} / \mathrm{d} \Omega$ emitted by the molecule on the metal:

$$
\frac{\mathrm{d} P_{\mathrm{rad}}^{\mathrm{SERS}-\mathbf{P}_{\mathrm{R}}}}{\mathrm{d} \Omega}=\underbrace{L_{\mathrm{m}} C_{E}^{2} \omega^{4}|\hat{\alpha}|^{2}}_{\mathrm{d}_{\Omega} \sigma} \cdot \underbrace{\left|\hat{\mathbf{E}}_{\mathrm{loc}}^{\mathbf{P}_{\mathrm{R}}} \cdot \hat{\alpha}_{\mathrm{N}} \cdot \hat{\mathbf{E}}_{\mathrm{loc}}^{\mathbf{P}_{\mathrm{L}}}\right|^{2}}_{\text {SMEF }} \cdot p^{\mathrm{inc}}
$$

This will implicitly yield the directional SMEF, an expression that is important for surface selection rules SSR.

While Raman scattering is an instantaneous process in which we profit from an enhancement of both the incoming and the outgoing radiation, fluorescence is a two step process. In consequence, even though the local field enhancement at the molecule's position translates into an increased excitation, the outgoing fluorescence itself will not be enhanced. Furthermore the presence of the metal will allow the outgoing radiation to couple to local surface-plasmon which increases the non-radiative decay rate. The combination of these two effects is often referred to as fluorescence quenching, an effect that will be exploited in Chapter 2.

\subsubsection{Surface enhanced Raman scattering}

As in the previous section it is possible to approach the problem of a molecule with metal objects in its close vicinity classically: the light scattered inelastically at the Raman frequency $\omega_{\mathrm{R}}$ can be modeled as the radiation coming from a dipole $\mathbf{p}\left(\omega_{\mathrm{R}}\right)$ induced by the local electric field $\mathbf{E}_{\text {loc }}^{\mathbf{P}_{\mathrm{L}}}\left(\omega_{\mathrm{L}}\right)$ :

$$
\mathbf{p}\left(\omega_{\mathrm{R}}\right)=L_{\mathrm{m}}^{1 / 4} \cdot \hat{\alpha}_{\mathrm{R}}\left(\omega_{\mathrm{L}}, \omega_{\mathrm{R}}\right) \cdot \mathbf{E}_{\mathrm{loc}}^{\mathbf{P}_{\mathrm{L}}}\left(\omega_{\mathrm{L}}\right)
$$

It is important to emphasize at this point that due to the presence of metallic objects, the local electric field $\mathbf{E}_{\text {loc }}^{\mathbf{P}_{\mathrm{L}}}$ can be much bigger in magnitude than the incoming, macroscopic field $\mathbf{E}^{\mathbf{P}_{\mathrm{L}}}$ from the laser. Furthermore $\mathbf{E}_{\text {loc }}^{\mathbf{P}_{\mathrm{L}}}$ is also very sensitive to the laser's polarization $\mathbf{P}_{\mathrm{L}}$ with respect to the metal surface (either transverse magnetic (TM) transverse electric (TE))

In order to calculate the far field $\mathbf{E}_{\text {far }}^{\mathbf{P}_{\mathrm{R}}}$ (and therefore the differential power) of $\mathbf{p}$ with metal objects in its close vicinity and a detection polarization $\mathbf{P}_{\mathrm{R}}$ characterized by $\mathbf{e}_{\mathbf{P}_{\mathrm{R}}} \in \operatorname{span}\left(\mathbf{e}_{\theta}, \mathbf{e}_{\phi}\right)$ located at 
$\mathbf{r}$ (see Fig. 7), we need to use the reciprocity theorem (page 240, [2]). If we imagine the dipole $\mathbf{p}$ located at the origin, the theorem puts this dipole's electric field $\mathbf{E}$ in a point $\mathbf{r}$ in relation to the electric field which a virtual dipole $\mathbf{p}^{\text {vir }}$ in $\mathbf{r}$ would create at the origin $\mathbf{O}$ :

$$
\mathbf{E} \cdot \mathbf{p}^{\mathrm{vir}}=\mathbf{E}^{\mathrm{vir}} \cdot \mathbf{p}
$$

If we now choose a normalized virtual dipole $\mathbf{p}^{\text {vir }}=\mathbf{e}_{\mathbf{P}_{\mathrm{R}}}$ along the detection polarization and set $\mathbf{r}$ large enough to consider a plane wave solution $\mathbf{E}_{\text {loc }}^{\mathrm{PW}-\text { vir }}$ for $\mathbf{E}^{\mathrm{vir}}$ with the metal objects and $\mathbf{E}^{\mathrm{PW} \text { - vir }}$ for $\mathbf{E}^{\mathrm{vir}}$ without them at the origin $\mathbf{0}$, then we obtain directly:

$$
\begin{aligned}
& \mathbf{E} \cdot \mathbf{e}_{\mathbf{P}_{\mathrm{R}}} \stackrel{\text { eq.1.40 }}{=} \quad \mathbf{E}_{\mathrm{loc}}^{\mathrm{PW}-\mathrm{vir}} \cdot \mathbf{p} \\
& =\frac{\mathbf{E}_{\text {loc }}^{\mathrm{PW}-\text { vir }}}{\left|\mathbf{E}^{\mathrm{PW}-\text { vir }}\right|} \cdot \mathbf{p} \cdot\left|\mathbf{E}^{\mathrm{PW}-\text { vir }}\right| \\
& =\underbrace{\left(\hat{\mathbf{E}}_{\text {loc }}^{\mathrm{PW}-\mathrm{vir}} \cdot \mathbf{e}_{\mathbf{p}}\right)}_{\text {term } 1} \cdot \underbrace{|\mathbf{p}|\left|\mathbf{E}^{\mathrm{PW}-\mathrm{vir}}\right|}_{\text {term } 2}
\end{aligned}
$$

In order to get a better understanding of this equation, let us look at its two terms more carefully: term 2 is the the maximum electric field strength that an oscillating dipole $\mathbf{p}$ in $\mathbf{r}$ would induce at the origin without any metallic objects. If it was only for this term, the differential power $\mathrm{d} P_{\mathrm{rad}}^{\mathrm{SERS}-\mathbf{P}_{\mathrm{R}}} / \mathrm{d} \Omega$ of the enhanced dipole $\mathbf{p}$ with a polarizer $\mathbf{P}_{\mathrm{R}}$ in front of the detector would already be given by the maximum differential power $\mathrm{d} P_{\mathrm{rad}}^{\mathrm{FD}-\max } / \mathrm{d} \Omega=C_{p} C_{E}^{2} \omega^{4}|\mathbf{p}|^{2}$ of a free dipole as given in eq. 1.17. Naturally, this expression for the free dipole has to be corrected by (term 1$)^{2}$ (since $P_{\mathrm{rad}} \propto|\mathbf{E}|^{2}$ ) and the local field correction factor $\sqrt{L_{\mathrm{m}}}$ (see subsection 1.2.5):

$$
\frac{\mathrm{d} P_{\mathrm{rad}}^{\mathrm{SERS}-\mathbf{P}_{\mathrm{R}}}}{\mathrm{d} \Omega}=\underbrace{\sqrt{L_{\mathrm{m}}} C_{p} C_{E}^{2} \omega^{4}|\mathbf{p}|^{2}}_{\mathrm{d}_{\Omega} P_{\mathrm{rad}}^{\mathrm{FD}-\max }} \cdot \underbrace{\left|\hat{\mathbf{E}}_{\mathrm{loc}}^{\mathrm{PW}-\mathrm{vir}} \cdot \mathbf{e}_{\mathbf{p}}\right|^{2}}_{M_{\mathrm{rad}}^{\mathbf{P}_{\mathrm{R}}}(\Omega)}
$$

Here we readily identified the polarized directional radiative enhancement-factor $M_{\mathrm{rad}}^{\mathbf{P}_{\mathrm{R}}}(\Omega)$ which is the ratio of the power emitted by a dipole of magnitude $|\mathbf{p}|$ with and without metallic objects:

$$
M_{\mathrm{rad}}^{\mathbf{P}_{\mathrm{R}}}(\Omega) \equiv \frac{\mathrm{d}_{\Omega} P_{\mathrm{rad}}^{\mathrm{SERS}-\mathbf{P}_{\mathrm{R}}}}{\mathrm{d}_{\Omega} P_{\mathrm{rad}}^{\mathrm{FD}-\max }}=\left|\hat{\mathbf{E}}_{\mathrm{loc}}^{\mathrm{PW}-\mathrm{vir}} \cdot \mathbf{e}_{\mathbf{p}}\right|^{2}
$$

In order to understand $M_{\text {rad }}^{\mathbf{P}_{\mathrm{R}}}(\Omega)$ better, let us replace the normalized local Plane-Wave solution $\hat{\mathbf{E}}_{\text {loc }}^{\text {PW }}$ vir of a virtual dipole $\mathbf{p}_{\text {vir }} \equiv \mathbf{e}_{\mathbf{P}_{\mathrm{R}}}$ with the normalized local electric field $\hat{\mathbf{E}}_{\text {loc }}^{\mathbf{P}_{\mathrm{R}}}$ of an incoming plane wave polarized along $\mathbf{P}_{R}$. The latter is obviously equivalent to the former but much easier to envision: instead of dealing with a virtual dipole, we can simply consider an incoming laser-beam with polarization $\mathbf{P}_{\mathrm{R}}$.

Now it is quite obvious, that the radiative enhancement-factor $M_{\mathrm{rad}}^{\mathbf{P}_{\mathrm{R}}}(\Omega)$ is the definition of the local field enhancement factor $M_{\mathbf{e}_{\mathbf{p}}}^{\mathbf{P}_{\mathrm{R}}}$ :

$$
M_{\mathrm{rad}}^{\mathbf{P}_{\mathrm{R}}}(\Omega)=\left|\hat{\mathbf{E}}_{\text {loc }}^{\mathbf{P}_{\mathrm{R}}} \cdot \mathbf{e}_{\mathrm{p}}\right|^{2} \equiv M_{\mathbf{e}_{\mathrm{p}}}^{\mathbf{P}_{\mathrm{R}}}
$$

This is a remarkable insight: the reciprocity theorem allows us to understand the emission of an enhanced dipole simply by solving the excitation problem (which can be much easier e.g. for a flat metal surface). 
In most common situations, $\mathbf{P}_{\mathrm{R}}$ is either TM or TE and the dipole orientation $\mathbf{e}_{\mathbf{p}}$ is commonly either $\perp$ (perpendicular) or $\|$ (parallel) to the metallic surface:

$$
M_{\perp}^{\mathbf{P}_{\mathrm{R}}} \equiv\left|\hat{\mathbf{E}}_{\text {loc }}^{\mathbf{P}_{\mathrm{R}}} \cdot \mathbf{e}_{\mathrm{z}}\right|^{2} \quad \text { and } \quad M_{\|}^{\mathbf{P}_{\mathrm{R}}} \equiv\left|\hat{\mathbf{E}}_{\text {loc }}^{\mathbf{P}_{\mathrm{R}}} \cdot \mathbf{e}_{\mathrm{x}}\right|^{2}+\left|\hat{\mathbf{E}}_{\mathrm{loc}}^{\mathbf{P}_{\mathrm{R}}} \cdot \mathbf{e}_{\mathrm{y}}\right|^{2}
$$

For flat surfaces $M$ can easily be calculated with the SPlaC program (p.567, [2]). It is the ratio of the enhanced local field projected onto a certain direction and the absolute value of the local field without any metal objects. Alternatively one could eliminate the normalization factor by considering an incoming field-strength of 1 .

For completeness (and for future reference) the total local field enhancement factor $M_{\text {loc }}^{\mathbf{P}_{\mathrm{R}}}$ shall be introduced at this point:

$$
M_{\mathrm{loc}}^{\mathbf{P}_{\mathrm{R}}} \equiv\left|\hat{\mathbf{E}}_{\mathrm{loc}}^{\mathbf{P}_{\mathrm{R}}}\right|^{2}=M_{\perp}^{\mathbf{P}_{\mathrm{R}}}+M_{\|}^{\mathbf{P}_{\mathrm{R}}}
$$

For simplicity the dependency of $M$ on the frequency $\omega$ and direction $\Omega$ has been omitted.

Since $P_{\text {rad }} \propto|\mathbf{E}|^{2}$, the maximum differential power (as given in eq. 1.17) for a free dipole $\mathbf{p}$ has to be corrected by (term 1$)^{2}=M_{\mathbf{e}_{\mathrm{p}}}^{\mathbf{P}_{\mathrm{R}}}$ of eq. 1.41 and by the local field correction factor $L_{\mathrm{m}}$ (see subsection 1.2.5):

$$
\begin{aligned}
& \frac{\mathrm{d} P_{\mathrm{rad}}^{\mathrm{SERS}-\mathbf{P}_{\mathrm{R}}}}{\mathrm{d} \Omega}=\sqrt{L_{\mathrm{m}}} C_{p} C_{E}^{2} \omega^{4}|\mathbf{p}|^{2} \cdot M_{\mathbf{e}_{\mathbf{p}}}^{\mathbf{P}_{\mathrm{R}}} \\
& \stackrel{\text { eq.1.44 }}{=} \sqrt{L_{\mathrm{m}}} C_{p} C_{E}^{2} \omega^{4} \quad\left|\hat{\mathbf{E}}_{\mathrm{loc}}^{\mathbf{P}_{\mathrm{R}}} \cdot \mathbf{p}\right|^{2} \\
& \stackrel{\text { eq.1.39 }}{=} \quad L_{\mathrm{m}} C_{p} C_{E}^{2} \omega^{4} \quad\left|\hat{\mathbf{E}}_{\mathrm{loc}}^{\mathbf{P}_{\mathrm{R}}} \cdot \hat{\alpha}_{\mathrm{R}} \cdot \mathbf{E}_{\mathrm{loc}}^{\mathbf{P}_{\mathrm{L}}}\right|^{2} \\
& =\underbrace{L_{\mathrm{m}} C_{E}^{2} \omega^{4}|\hat{\alpha}|^{2}}_{\equiv \mathrm{d}_{\Omega} \sigma} \underbrace{\left|\hat{\mathbf{E}}_{\text {loc }}^{\mathbf{P}_{\mathrm{R}}} \cdot \hat{\alpha}_{\mathrm{N}} \cdot \hat{\mathbf{E}}_{\mathrm{loc}}^{\mathbf{P}_{\mathrm{L}}}\right|^{2}}_{\text {SMEF } F} \underbrace{\left|\mathbf{E}^{\mathrm{inc}}\right|^{2} C_{p}}_{p^{\text {inc }}}
\end{aligned}
$$

Here the middle term is the SMEF $F$. Since the SERS cross-section $\mathrm{d}_{\Omega} \sigma^{\text {SERS }}$ is defined as the ratio of the emitted power and the incoming power density $p^{\text {inc }}$ we can conclude:

$$
\frac{\mathrm{d} \sigma_{\text {rad }}^{\text {SER }}}{\mathrm{d} \Omega}=\underbrace{\left|\hat{\mathbf{E}}_{\mathrm{loc}}^{\mathbf{P}_{\mathrm{R}}}\left(\omega_{\mathrm{R}}, \Omega_{\mathrm{R}}\right) \cdot \hat{\alpha}_{\mathrm{N}} \cdot \hat{\mathbf{E}}_{\mathrm{loc}}^{\mathbf{P}_{\mathrm{L}}}\left(\omega_{\mathrm{L}}, \Omega_{\mathrm{L}}\right)\right|^{2}}_{\text {SMEF } F} \frac{\mathrm{d} \sigma_{\mathrm{rad}}^{\mathrm{R}}}{\mathrm{d} \Omega}
$$

In order to evaluate this very general expression let us consider BS configuration $\left(\Omega_{\mathrm{L}}=\Omega_{\mathrm{R}}\right.$ along the the $x$-axis) with an incoming laser polarization along the $z$-axis $\left(\mathbf{P}_{\mathrm{L}}=z\right)$. For this situation 2 extreme situations are feasible: one in which the detection polarization $\mathbf{P}_{\mathrm{R}}$ is parallel to $\mathbf{P}_{\mathrm{L}}$ and one in which it is perpendicular to $\mathbf{P}_{\mathrm{L}}$. For those two situations the SMEF in eq. 1.48 takes on the form:

$$
\begin{gathered}
\operatorname{SMEF}_{\mathrm{BS}}^{\|} \stackrel{\text { Def.1.46 }}{=} M_{\mathrm{loc}}^{z}\left(\omega_{\mathrm{R}}\right) M_{\mathrm{loc}}^{z}\left(\omega_{\mathrm{L}}\right) \cdot \underbrace{\left|\hat{\mathbf{e}}_{\mathrm{loc}}^{z}\left(\omega_{\mathrm{R}}\right) \cdot \hat{\alpha}_{\mathrm{N}} \cdot \hat{\mathbf{e}}_{\mathrm{loc}}^{z}\left(\omega_{\mathrm{L}}\right)\right|^{2}}_{\mathrm{T}_{\mathrm{BS}}^{\|}} \\
\mathrm{SMEF}_{\mathrm{BS}}^{\perp} \stackrel{\text { Def.1.46 }}{=} M_{\mathrm{loc}}^{y}\left(\omega_{\mathrm{R}}\right) M_{\mathrm{loc}}^{z}\left(\omega_{\mathrm{L}}\right) \cdot \underbrace{\left|\hat{\mathbf{e}}_{\mathrm{loc}}^{y}\left(\omega_{\mathrm{R}}\right) \cdot \hat{\alpha}_{\mathrm{N}} \cdot \hat{\mathbf{e}}_{\mathrm{loc}}^{z}\left(\omega_{\mathrm{L}}\right)\right|^{2}}_{\mathrm{T}_{\mathrm{BS}}^{\perp}}
\end{gathered}
$$


$T$ is called the SSR-factor and $\hat{\mathbf{e}}_{\text {loc }}$ stands for the unit-vector along the local field orientation. Quite naturally, for a situation with no detection polarization those two factors are simply added:

$$
\mathrm{SMEF}_{\mathrm{BS}}=\mathrm{SMEF}_{\mathrm{BS}}^{\|}+\mathrm{SMEF}_{\mathrm{BS}}^{\perp}
$$

If we now look at a simple situation in which the Raman tensor $\hat{\alpha}$ is isotropic and the local field orientations behave "well" (i.e. $\hat{\mathbf{e}}_{\mathrm{loc}}^{y}\left(\omega_{\mathrm{R}}\right) \perp \hat{\mathbf{e}}_{\mathrm{loc}}^{z}\left(\omega_{\mathrm{L}}\right)$ and $\left.\hat{\mathbf{e}}_{\mathrm{loc}}^{z}\left(\omega_{\mathrm{R}}\right) \| \hat{\mathbf{e}}_{\mathrm{loc}}^{z}\left(\omega_{\mathrm{L}}\right)\right)$ then eq. 1.51 simplifies considerably (p.246, [2]):

$$
\mathrm{SMEF}_{\mathrm{BS}}=M_{\mathrm{loc}}^{z}\left(\omega_{\mathrm{R}}\right) \cdot M_{\mathrm{loc}}^{z}\left(\omega_{\mathrm{R}}\right)
$$

For a random $z$ orientation, this is called the $|\mathbf{E}|^{4}$ approximation: it ignores polarization effects and corrections stemming from the surface selection rules factor $T$. Even though the assumptions we made before seem to be rather specific, this approximation proves to be good for most cases and hence eq. 1.48 can be approximated as:

$$
\frac{\mathrm{d} \sigma_{\mathrm{rad}}^{\mathrm{SERS}}}{\mathrm{d} \Omega} \approx \underbrace{M_{\mathrm{loc}}\left(\omega_{\mathrm{R}}\right) \cdot M_{\mathrm{loc}}\left(\omega_{\mathrm{L}}\right)}_{\mathrm{SMEF}} \cdot \frac{\mathrm{d} \sigma_{\mathrm{rad}}^{\mathrm{R}}}{\mathrm{d} \Omega}
$$

\subsubsection{Surface enhanced fluorescence}

When treated in detail, surface enhanced fluorescence is even more complex than SERS: on top of polarization effects and surface selection rules, one has to look at the irreversible relaxation process which separates the absorption from the emission and may or may not destroy memory of the incoming polarization. Since for the scope of this thesis a semi-quantitative understanding of enhanced fluorescence and fluorescence quenching is more than sufficient, we will draw from the detailed explanation of dipole excitation and emission given in the previous section and content ourselves with citing results that are based on an approximation analogous to the $|\mathbf{E}|^{4}$ one for the SERS case (i.e. polarization effects and SSRs are ignored).

As has already been explained, fluorescence is a two step process: in the first step a molecule absorbs a photon and is thereby excited into a higher electronic state $S_{1}$. Since absorption is a statistical process proportional to the number of incoming photons (i.e. the local light intensity), the modified absorption cross-section $\tilde{\sigma}^{\text {abs }}$ of a randomly oriented molecule with surrounding metal objects is simply its free (vacuum) absorption cross-section $\sigma^{\text {abs }}$ times the local field enhancement factor (see p.250, 4.80, [2]):

$$
\tilde{\sigma}^{\mathrm{abs}}=M_{\mathrm{Loc}} \cdot \sigma^{\mathrm{abs}}
$$

This is very similar to what happens in the SERS case for the excitation of the enhanced dipole.

After this enhanced absorption the molecule experiences a non-radiative relaxation to the lowest vibrational state in $S_{1}$ followed by the emission of a photon while relaxing into $S_{0}$. This is the second step of the fluorescence process and it is, unlike SERS, completely independent of the first: no matter how high the radiative enhancement factor $M_{\text {rad }}$, no more photons can be emitted 
than there are excited molecules. This means that even if there was no competition from nonradiative processes, the modified quantum yield $\tilde{Q}$ could not be greater than 1 . In consequence the modified fluorescence cross-section $\tilde{\sigma}^{\text {Fluo }}$ can be safely approximated as follows:

$$
\tilde{\sigma}^{\text {Fluo }} \stackrel{\text { eq.1.14 }}{=} \tilde{Q} \cdot \tilde{\sigma}^{\text {abs }} \stackrel{\text { eq. } 1.54}{\leq} 1 \cdot M_{\mathrm{Loc}} \cdot \sigma^{\text {abs }}=\frac{M_{\text {Loc }}}{Q} \cdot \underbrace{Q \cdot \sigma^{\text {abs }}}_{\sigma^{\text {Fluo }}}
$$

From this equation an upper limit for the fluorescence enhancement-factor $\mathrm{SMEF}_{\text {Fluo }}:=\tilde{\sigma}^{\text {Fluo }} / \sigma^{\text {Fluo }}$ can be deduced:

$$
\mathrm{SMEF}_{\text {Fluo }} \leq \frac{M_{\mathrm{Loc}}}{Q} \quad \text { which compares to } \quad \mathrm{SMEF}_{\mathrm{R}} \stackrel{\text { eq.1.53 }}{=} M_{\mathrm{loc}} \cdot M_{\mathrm{rad}}
$$

This simple comparison already shows that for a common situation in which $Q \sim 1$ (as for RH6G) and $M_{\mathrm{rad}}=M_{\mathrm{loc}} \sim 10^{3}$ (by virtue of reciprocity), the enhancement for Raman scattering outweighs by far the fluorescence enhancement.

In order to get a better approximation for the modified quantum yield $\tilde{Q}$, let us now have a closer look at the molecule on the metal surface in the lowest vibrational state of $S_{1}$. It can relax into the ground state through a radiative process (quantified by $\tilde{\Gamma}^{\mathrm{rad}}=M_{\mathrm{rad}} \cdot \Gamma^{\mathrm{rad}}$ ) or a non-radiative process. The latter can either happen through the known channel $\Gamma^{\mathrm{NR}}$ or the emission of a photon that is subsequently absorbed by the metal and quantified by $\tilde{\Gamma}_{\mathrm{EM}}^{\mathrm{NR}}$. In many cases $\tilde{\Gamma}^{\mathrm{rad}}+\tilde{\Gamma}_{\mathrm{EM}}^{\mathrm{NR}}$ is much bigger than $\Gamma^{\mathrm{NR}}$ and we can approximate (p251, 4.82):

$$
\tilde{Q}=\frac{\tilde{\Gamma}^{\mathrm{rad}}}{\tilde{\Gamma}^{\mathrm{rad}}+\tilde{\Gamma}_{\mathrm{EM}}^{\mathrm{NR}}+\Gamma^{\mathrm{NR}}} \approx \frac{\tilde{\Gamma}^{\mathrm{rad}}}{\tilde{\Gamma}^{\mathrm{rad}}+\tilde{\Gamma}_{\mathrm{EM}}^{\mathrm{NR}}}=\frac{M_{\mathrm{rad}}}{M_{\mathrm{tot}}}
$$

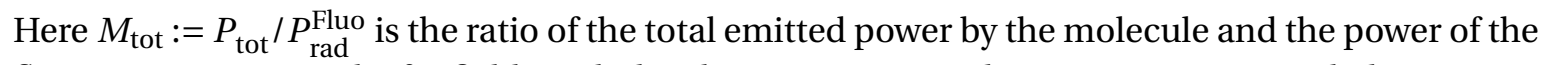
fluorescence seen in the far field. With the above equation and eq. 1.54 we can now deduce:

$$
\begin{aligned}
& \tilde{\sigma}^{\text {Fluo }}=\tilde{Q} \cdot \tilde{\sigma}^{\text {abs }} \approx \frac{M_{\mathrm{rad}}}{M_{\mathrm{tot}}} \cdot M_{\mathrm{loc}} \sigma^{\mathrm{abs}}=\frac{M_{\mathrm{Loc}} M_{\mathrm{rad}}}{Q \cdot M_{\mathrm{tot}}} \cdot \underbrace{Q \cdot \sigma^{\mathrm{abs}}}_{\sigma^{\text {Fluo }}} \\
& \Rightarrow \mathrm{SMEF}_{\text {Fluo }} \approx \frac{M_{\mathrm{Loc}} M_{\mathrm{rad}}}{Q \cdot M_{\mathrm{tot}}}
\end{aligned}
$$

This allows us now, to have a better look at the ratio of the enhancement-factors (eq. 1.53 and 1.59):

$$
\frac{\mathrm{SMEF}_{\mathrm{R}}}{\mathrm{SMEF}_{\text {Fluo }}}=Q \cdot M_{\mathrm{tot}} \quad \text { with } \quad M_{\mathrm{tot}}=1+\frac{3 \kappa}{16\left(k_{\mathrm{M}} d\right)^{3}} \operatorname{Im}\left\{\frac{\epsilon-\epsilon_{\mathrm{M}}}{\epsilon+\epsilon_{\mathrm{M}}}\right\}
$$

Here $M_{\mathrm{tot}}$ is taken without further derivation from p.303, eq. 6.13 in reference [2] ( $\kappa_{\perp}=2$ and $\kappa_{\|}=1$ and $k_{\mathrm{M}}=\frac{\omega}{c} \sqrt{\epsilon_{\mathrm{M}}}$ ). The $\frac{1}{d^{3}}$ dependency of $M_{\text {tot }}$ implies that the smaller the distance $d$ of the molecule to the metal surface, the more the enhancement of the Raman signal outweighs the fluorescence enhancement. 


\subsection{The SPP-resonance condition}

Surface Plasmon Polaritons (SPP) are a solution of Maxwell's equations at a planar interface between a metal like Ag or Au (with dielectric function $\epsilon_{\mathrm{m}}(\omega)$ ) and a dielectric medium like air or water (with dielectric function $\epsilon_{\mathrm{d}}(\omega)$ ). This solution can be interpreted as a electromagnetic surface mode that decays exponentially away from the surface but propagates along the interface with a wavevector $k_{x}$ that can be shown to have the form:

$$
k_{x}=k_{0} \sqrt{\frac{\epsilon_{\mathrm{m}} \epsilon_{\mathrm{d}}}{\epsilon_{\mathrm{m}}+\epsilon_{\mathrm{d}}}}
$$

The derivation of this dispersion relation (or resonance condition) is a standard exercise done in numerous textbooks and articles $[2,17,18]$ and is a natural outcome when looking at planar interfaces (see Fig 8) and their general, plane-wave solutions. A very concise and rigorous treatment (that adheres to a consistent sign convention and thereby avoids the pitfalls sometimes encountered in more "hand-waving" derivations) can be found in [2] on page 542 which we will follow closely in this section. The goal is to arrive at the SPP dispersion relation of eq. 1.61 and show that it corresponds to a physical wave that cannot be excited coming from the air / water side.

\subsubsection{Plane wave solution in a homogeneous medium}

The plane-wave solutions of Maxwell's equations in a homogeneous medium with dielectric constant $\epsilon$ are given by ([2], p.532):

$$
\begin{aligned}
\mathbf{E} & =\operatorname{Re}\left\{\mathbf{E}_{0} \exp [i(\mathbf{k} \circ \mathbf{x}-\omega t]\}\right. \\
\mathbf{H} & =\operatorname{Re}\left\{\mathbf{H}_{0} \exp [i(\mathbf{k} \circ \mathbf{x}-\omega t]\}\right.
\end{aligned}
$$

The following relations are dictated by Maxwell's equations:

$$
\begin{array}{llrl}
\mathbf{k} \circ \mathbf{k}=\epsilon(\omega) k_{0}^{2} & \text { with } & k_{0}:=\omega / c \\
\mathbf{n} \circ \mathbf{n}=1 & \text { with } & \mathbf{n}:=\mathbf{k} / k
\end{array}
$$

Here we used the definition $k:=\sqrt{\mathbf{k} \circ \mathbf{k}}$. Note that $\mathbf{k}$ can be complex and that therefore in general $k \neq|\mathbf{k}|$. Furthermore the electric and magnetic field components are perpendicular to each other:

$$
\begin{array}{rll}
\mathbf{E}_{0} & \propto \mathbf{n} \times \mathbf{H}_{0} \\
\mathbf{H}_{0} & \propto \mathbf{n} \times \mathbf{E}_{0}
\end{array}
$$

\subsubsection{Plane wave solution in two homogeneous media}

For the situation of two homogeneous media separated by a planar interface all solutions can be written as a superposition of plane waves. This is a simple consequence of the linearity of Maxwell's equations. Furthermore it is straight forward to show the following:

- that any solution can be written as a superposition of 4 plane waves of which each is characterized by its $\mathbf{k}$-vector as displayed in Fig. 8 . 
- that due to translational symmetry the $k_{x}$-component is conserved :

$$
k_{i, x}=\tilde{k}_{i, x}=: k_{x} \text { for } i=1,2
$$

- that without loss of generality the $k_{y}$-component can be assumed to vanish:

$$
k_{i, y}=\tilde{k}_{i, y}=0 \text { for } i=1,2
$$

- that for the $k_{z}$-component the following relation holds true:

$$
k_{i, z}=-\tilde{k}_{i, z} \stackrel{\text { eq.1.64 }}{=} \pm \sqrt{\epsilon_{i} k_{0}^{2}-k_{x}^{2}} \text { for } i=1,2
$$

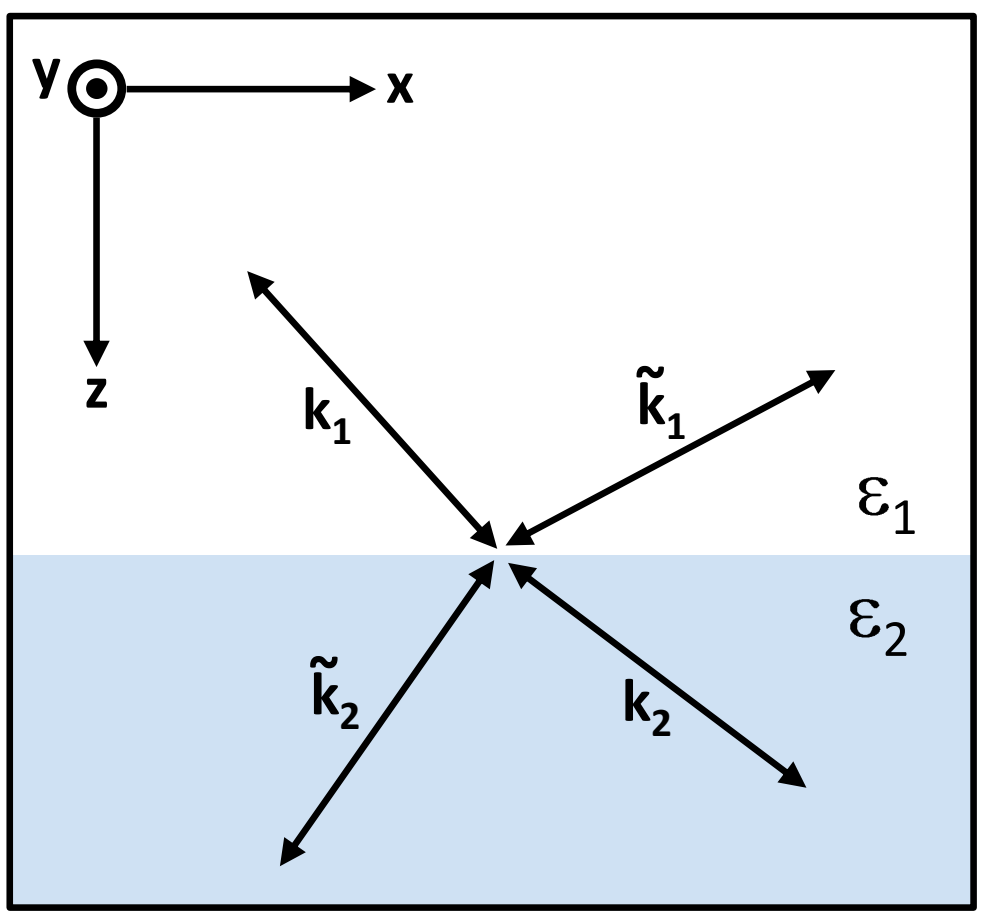

Fig. 8:

Schematic illustration of four plane-wave solutions at the boundary of two dielectric media. Their superposition constitutes the most general plane-wave solution at a planar interface.

\subsubsection{Sign-convention for plane-wave solutions}

Even though a sign-convention may seem technical, it will prove invaluable for distinguishing physical solutions of Maxwell's equations from non-physical ones. Let us therefore make the following convention:

- If medium 1 is transparent (i.e. $\operatorname{Im}\left\{\epsilon_{1}(\omega)\right\}=0$ ), an incoming light-source (i.e. $\operatorname{Re}\left\{k_{1, z}\right\}>0$ ) present at $-\infty$ shall be described by $\mathbf{E}_{1}$ rather than $\tilde{\mathbf{E}}_{1}$

Otherwise (i.e. for a non-transparent medium,1) no incident source shall be considered (it would be unphysical) and the scattered wave (described by $\tilde{\mathbf{E}}_{1}$ ) shall be evanescent (i.e. $\left.\operatorname{Im}\left\{\tilde{k}_{1, z}\right\} \stackrel{\text { eq. }}{=} \operatorname{Im}\left\{-k_{1, z}\right\}<0\right)$. 
In short this can be written as:

$$
\text { If } \begin{aligned}
\operatorname{Im}\left\{k_{1, z}\right\}=0 \text { then } \operatorname{Re}\left\{k_{1, z}\right\} & >0 \\
\text { else } \operatorname{Im}\left\{k_{1, z}\right\} & >0 \text { and } \mathbf{E}_{1}=0
\end{aligned}
$$

- Similarly, if medium 2 is transparent (i.e. $\operatorname{Im}\left\{\epsilon_{2}(\omega)\right\}=0$ ), an incoming light-source (i.e. $\operatorname{Re}\left\{\tilde{k}_{2, z}\right\}<$ 0 ) present at $+\infty$ shall be described by $\tilde{\mathbf{E}}_{2}$ rather than $\mathbf{E}_{2}$

Otherwise (i.e. for a non-transparent medium 2) no incident source shall be considered (it would be unphysical) and the scattered wave (described by $\mathbf{E}_{2}$ ) shall be evanescent (i.e. $\operatorname{Im}\left\{k_{2, z}\right\}>0$ ).

In short this can be written as:

$$
\text { If } \begin{aligned}
\operatorname{Im}\left\{k_{2, z}\right\}=0 \text { then } \operatorname{Re}\left\{k_{2, z}\right\} & >0 \\
\text { else } \operatorname{Im}\left\{k_{2, z}\right\} & >0 \text { and } \tilde{\mathbf{E}}_{2}=0
\end{aligned}
$$

\subsubsection{Boundary conditions}

It is possible to show that any plane-wave solution can be written as a sum of transverse magnetic (TM) and transverse electric (TE) solutions and that furthermore the polarization is conserved at the planar interface. It is therefore sufficient to only treat the case of TM and TE polarization for which the boundary conditions can be written elegantly in matrix form:

$$
\left(\begin{array}{l}
F_{1 y} \\
\tilde{F}_{1 y}
\end{array}\right)=\left(\begin{array}{cc}
1+K & 1-K \\
1-K & 1+K
\end{array}\right)\left(\begin{array}{l}
F_{2 y} \\
\tilde{F}_{2 y}
\end{array}\right)
$$

Here $F \equiv H$ and $K=\frac{\epsilon_{1} k_{2, z}}{\epsilon_{2} k_{1, z}}$ for the case of TM polarization while $F \equiv E$ and $K=\frac{k_{2, z}}{k_{1, z}}$ for the case of TE polarization.

\subsubsection{SPP resonance condition}

With this background it is now sensible to ask if (for a given frequency $\omega$ ) there is a non-trivial solution that fulfills the boundary conditions in eq. 1.75 with no incoming light $\left(\mathbf{E}_{1}=\tilde{\mathbf{E}}_{2} \stackrel{!}{=} 0\right)$ i.e. if there is a $K$ for which the following equation holds true:

$$
\left(\begin{array}{c}
0 \\
\tilde{F}_{1 y}
\end{array}\right) \stackrel{\text { eq.1.75 }}{=}\left(\begin{array}{l}
F_{2 y} \cdot(1+K) \\
F_{2 y} \cdot(1-K)
\end{array}\right)
$$

Using the expressions for $K^{\mathrm{TM}}$ and $K^{\mathrm{TE}}$ from page 544 and 545 in [2] this necessitates that:

$$
K=-1 \Longleftrightarrow\left\{\begin{array}{cr}
\epsilon_{2} k_{1, z}=-\epsilon_{1} k_{2, z} & \text { for TM polarization } \\
\epsilon_{2}=\epsilon_{1} & \text { for TE polarization }
\end{array}\right.
$$

The case for TE polarization is simple to interpret: a non-trivial solution exists only for the case $\epsilon_{2}=\epsilon_{1}$ which means that from an optical point of view medium 1 and medium 2 are the same, i.e. 
there is no interface. This solution is not interesting for us. However the TM-case is non-trivial and therefore interesting for us. A necessary (but not sufficient) condition then is:

$$
\begin{aligned}
\left(\epsilon_{2} k_{1, z}\right)^{2} & =\left(\epsilon_{1} k_{2, z}\right)^{2} \\
\stackrel{\text { eq. 1.70 }}{\Longleftrightarrow} \epsilon_{2}^{2} \cdot\left(\epsilon_{1} k_{0}^{2}-k_{x}^{2}\right) & =\epsilon_{1}^{2} \cdot\left(\epsilon_{2} k_{0}^{2}-k_{x}^{2}\right) \\
\Longleftrightarrow k_{x}^{2}\left(\epsilon_{1}^{2}-\epsilon_{2}^{2}\right) & =k_{0}^{2}\left(\epsilon_{1}^{2} \epsilon_{2}-\epsilon_{2}^{2} \epsilon_{1}\right) \\
\Longleftrightarrow k_{x} & = \pm k_{0} \sqrt{\frac{\epsilon_{1} \epsilon_{2}}{\epsilon_{1}+\epsilon_{2}}}
\end{aligned}
$$

By convention we choose $k_{x}$ to be positive which determines in turn (see eq. 1.70) $\tilde{k}_{1, z}$ and $k_{2, z}$. Now it is important to check that

1. $\tilde{k}_{1, z}$ and $k_{2, z}$ have a sign that corresponds to a physical solution (i.e. a sign that respects the conventions in eq. 1.71 - 1.74).

2. $K^{\mathrm{TM}}=-1$ rather than $K^{\mathrm{TM}}=+1$

Rather than checking those two conditions for the most general case, lets have a look at the case most relevant to us: the interface between a dielectric like air $\left(\epsilon_{1} \equiv \epsilon_{\text {air }}=1\right)$ and a metal like Ag $\left(\epsilon_{2} \equiv\right.$ $\left.\epsilon_{\mathrm{Ag}}=-16+i 0.7 @ \lambda=350 \mathrm{~nm}\right)$. Since $\left|\operatorname{Re}\left\{\epsilon_{\mathrm{Ag}}\right\}\right| \gg\left|\operatorname{Im}\left\{\epsilon_{\mathrm{Ag}}\right\}\right|$ it is reasonable (in a first approximation) to assume medium 2 to be loss free (i.e. $\epsilon_{2} \approx-16$ ).

Inserting the expression for $k_{x}$ in eq. 1.81 into the expression for $k_{z}$ in eq. 1.70 leaves us with a choice of sign. Let us consider

$$
\begin{aligned}
& k_{1, z}=+k_{0} \epsilon_{1} \sqrt{\frac{1}{\epsilon_{1}+\epsilon_{2}}} \\
& k_{2, z}=-k_{0} \epsilon_{2} \sqrt{\frac{1}{\epsilon_{1}+\epsilon_{2}}}
\end{aligned}
$$

It is easy to see that this indeed corresponds to a physical solution satisfying eq. $1.71-1.74$ and that it also fulfills $K=-1$.

This means that for the idealized case of a loss free Ag-air interface it is indeed possible to have a solution that is the superposition of 2 evanescent waves (in the z-direction) traveling with $k_{x}=$ $k_{0} \sqrt{\frac{\epsilon_{1} \epsilon_{2}}{\epsilon_{1}+\epsilon_{2}}} \approx 1.03 \cdot k_{0}$ (in the x-direction). This solution is called a Surface Plasmon Polariton and it exists (by definition) with no incoming light.

It is clear from the dispersion relation that it cannot be excited coming from air as the resonance condition for $k_{x}$ is slightly bigger than $k_{0}$. This means that for its excitation one has to come from the metal side and use a $k$-booster which can e.g. be a glass prism.

It is also interesting to note that $k_{x}^{\mathrm{SPP}}$ is just beyond the condition for total internal reflection (TIR) on a glass-air interface: $k_{x}^{\mathrm{TIR}}=k_{0}$.

With further, rather tedious considerations it is possible to show that SPPs not only exist for the idealized case of a loss free metal but also for the case of real metals. 


\subsection{Experimental methods}

Having introduced some basic optical effects in the last section, it is now important to briefly explain which samples were used to observe them and the instruments that collected the data.

\subsubsection{Samples}

For the experiments presented in this thesis we confined our attention to 3 different probes, all of them being dyes. The latter are important molecules used in biology (DNA sequencing), physics (gain medium for dye lasers, flow tracing), food processing, colouring of wood, paper, cotton and many other areas; While most of their commercial use is related to their strong absorption / fluorescence behavior in the visible (the former gives them the nice colour we are seeing), they are of great interest to us because of their large Raman cross-sections, which makes it easy to detect them.

- RH6G is one of the most commonly used dyes and is highly resonant in the green. Because of its large quantum yield $(\approx 1)$, its Raman spectrum is drowned in the fluorescence background for this wavelength.

- Crystal Violet (CV) is also resonant in the visible but its fluorescence process is very inefficient. This allows one to observe its Raman spectrum even at resonance.

- Nile Blue (NB) has its resonance in the red, an excitation wavelength extensively used in the second part of this thesis (Chapter 3 and 4). Since its Raman cross-section outperforms the one of RH6G and CV in that region, it was the dye of our choice for that part.

These dyes were either dissolved in water or deposited on SERS substrates such as:

- a Ag Lee \& Meisel colloid: those colloids were prepared according to a standard procedure [19] and essentially consist of Ag nano particles (average diameter about $50 \mathrm{~nm}$ ) in water.

- a Ag or Au metal film: those flat films $(50 \mathrm{~nm})$ on glass were initially produced here in New Zealand by evaporation. To obtain better quality we eventually shifted to commercially produced films from the company SSens in the Netherlands.

- a Klarite substrate: those substrates consist of a silicon template with a regular nano-pattern that is coated with gold (Au). See [20] for further details.

\subsubsection{Experimental setup}

For our experiments a He-Ne-laser and a tunable $\mathrm{Ar}^{+}-\mathrm{Kr}^{+}$-laser were available, providing the seven excitation lines $\left(\lambda_{\mathrm{L}}=458 \mathrm{~nm}, 488 \mathrm{~nm}, 514 \mathrm{~nm}, 532 \mathrm{~nm}, 568 \mathrm{~nm}, 633 \mathrm{~nm}, 647 \mathrm{~nm}\right)$ used to illuminate the samples. The sample in turn emits light (Rayleigh, Fluorescence, Raman - see previous sections) which is collected by an objective and imaged onto a pinhole (magnification-factor of $\times 40$ for a $\times 100$ objective). While it is the pinhole's purpose to serve as a spatial filter when closed 
sufficiently far, it was left wide open and therefore unused for all of our applications. The image of the sample at the pinhole-plane is then recreated on the entrance slit of the spectrometer ${ }^{3}$. This is schematically depicted in Fig. 9:

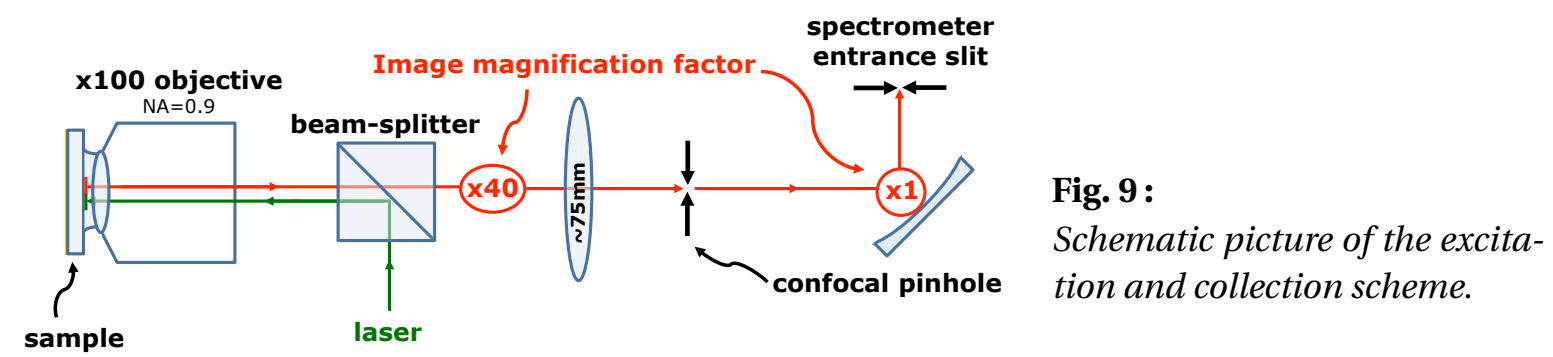

Most of the data presented in this thesis was taken with a T64000 triple subtractive Jobin-Yvon spectrometer equipped with a nitrogen-cooled CCD. For this type of spectrometer, the light arriving at the entrance slit is processed in three separate stages: in the first stage, a grating (usually with 600 or 1800 lines $/ \mathrm{mm}$ ) disperses it into its constituent colours. By changing the angle between the surface normal of the grating and the incoming beam, one can select which part of the spectrum passes through the intermediate slit and into the second stage. There the light is collimated onto the third stage entrance slit by a second grating arranged in an optical setup exactly symmetric to the one in the first stage. This double subtractive configuration filters out any (unwanted) laser-light and allows stage 3 to do the actual spectroscopy: again a grating breaks up the light which gets finally collected by a CCD array of $256 \times 1024$ pixels. This layout is schematically illustrated in Fig. 10.

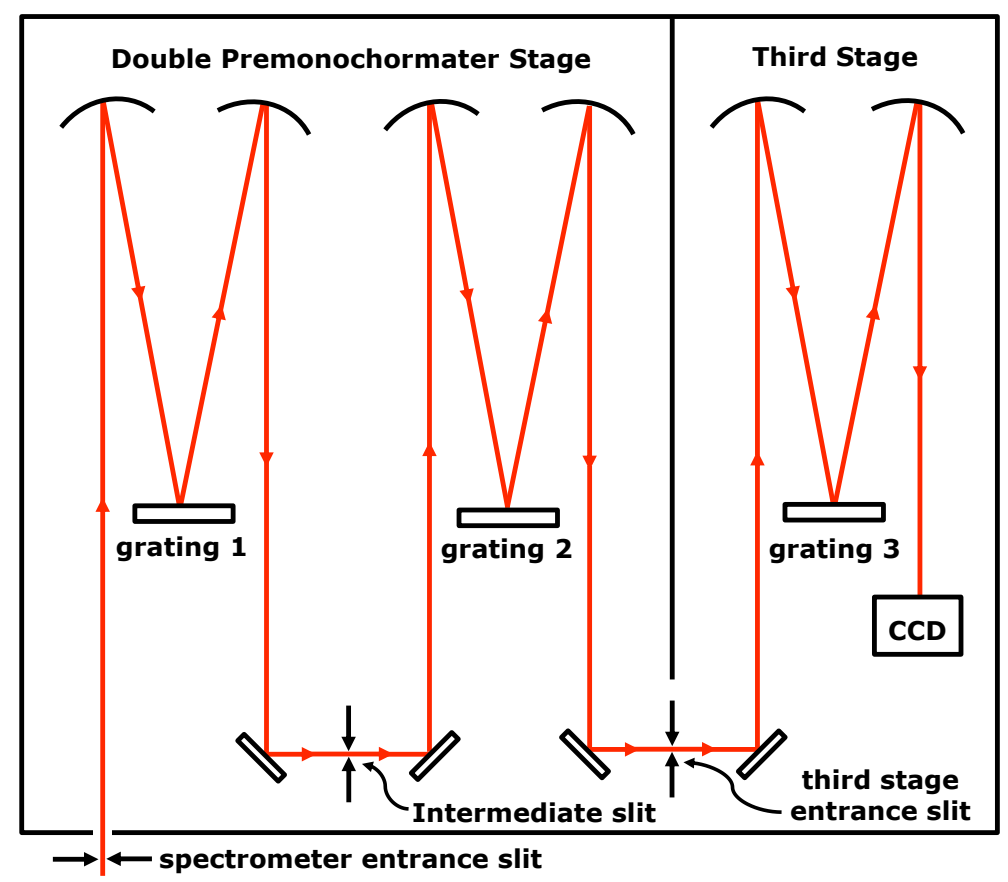

Fig. 10:

Schematic picture of the triple spectrometer used for our experiments.

While the CCD's $y$-axis (with 256 pixels) corresponds to an actual physical dimension on the sample, the CDD's $x$-axis corresponds to a certain Raman-wavelength $\lambda_{\mathrm{R}}(x)$. Usually all counts from pixels with the same $x$-coordinate (i.e. with the same Raman wavelength) are binned resulting in

\footnotetext{
${ }^{3}$ the size of this slit is important for the spectral resolution of the data
} 
a signal $S\left(\lambda_{\mathrm{R}}\right)$. It is common to display this signal not as a function of $\lambda_{\mathrm{R}}$ but instead as a function of the Raman shift $v$ :

$$
\begin{aligned}
v:=\frac{1}{\lambda_{\mathrm{L}}}-\frac{1}{\lambda_{\mathrm{R}}} & =\frac{1}{h c} \cdot\left(\frac{h c}{\lambda_{\mathrm{L}}}-\frac{h c}{\lambda_{\mathrm{R}}}\right) \\
& =\frac{1}{h c} \cdot\left(E_{\mathrm{L}}-E_{\mathrm{R}}\right)
\end{aligned}
$$

Using customary notation $h \approx 6.6 \times 10^{-34} \mathrm{~J} \cdot \mathrm{sec}$ is the Planck constant and $c \approx 3.0 \times 10^{8} \frac{\mathrm{m}}{\mathrm{sec}}$ the vacuum speed of light. It is apparent that the definition of $v$ is such that it is proportional to the energy-difference of incident and scattered photon, a convenient choice since in that format a peak corresponding to a certain molecular vibration will always appear at the same position regardless of the excitation wavelength $\lambda_{\mathrm{L}}$.

It is worth pointing out that in this setup the intermediate slit fulfills the task of an ordinary notchfilter by blocking the scattered light of the laser which would otherwise obscure the desired Raman spectrum. But where an ordinary notch-filter works only for a single excitation wavelength in the visible and cuts out not only the laser-line at $\lambda_{\mathrm{L}}$ itself but also anything within a spectral range of about $100 \mathrm{~cm}^{-1}$ around that laser line, a triple spectrometer works for any excitation wavelength and allows us to observe Raman scattered light as close as about $1 \mathrm{~cm}^{-1}$ or $2 \mathrm{~cm}^{-1}$ to the laserline. This advantage in flexibility of a triple spectrometer outweighs in most situations by far the disadvantage of being slightly less efficient due to more optical components. 


\section{Chapter 2}

\section{Measuring resonant Raman cross-sections}

The work discussed in this chapter was published in "Journal of Physical Chemistry A", 2010 [21].

\subsection{Basic idea}

Resonant Raman cross-sections are notoriously difficult to obtain because in most cases the Raman spectrum of the molecule under consideration is swamped in a huge fluorescence background. However, by placing the molecule on a metal surface, a process called fluorescence quenching suppresses the background and allows one to observe a clear Raman spectrum.

In this chapter a new method is proposed, that allows a quantitative analysis of the SERS spectra: by comparing the SERS signal of an unknown molecule (in our case Rhodamine6G) with the SERS signal of a molecule with known cross-section (Crystal Violet), we were able to deduce the resonant Raman cross-sections from the relative peak intensities.
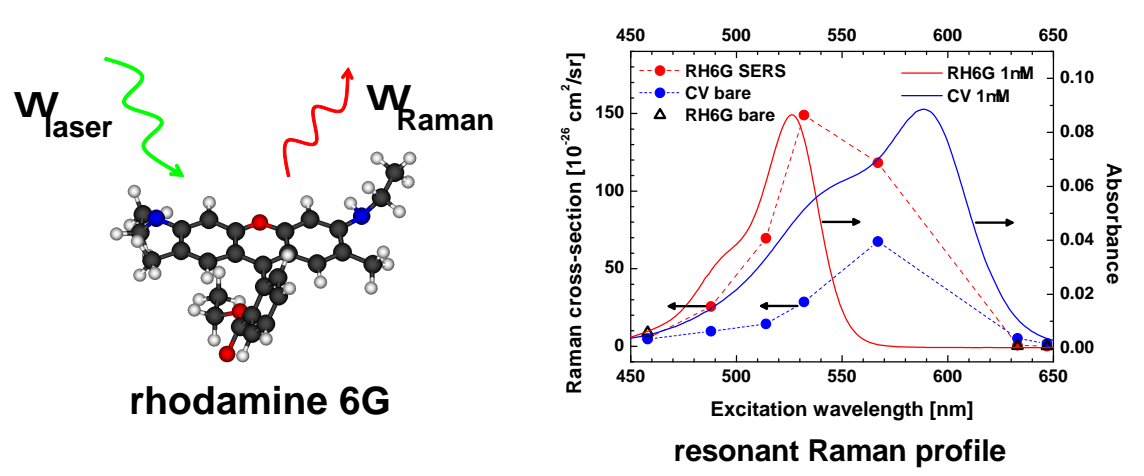

Fig. 11 : a) Chemical structure of Rhodamine $6 G\left(\mathrm{C}_{28} \mathrm{H}_{31} \mathrm{~N}_{2} \mathrm{O}_{3}\right)$, the molecule of interest for this chapter (black: carbon, white: hydrogen, blue: nitrogen, red: oxygen) b) key finding of this chapter: the resonant Raman cross-sections of Rhodamine $6 G$ for 7 excitation wavelengths across the visible spectrum. 


\subsection{Context}

The determination of Raman cross sections $[2,14]$ is a textbook-like example of molecular spectroscopy [22, 23]. However, differential resonant Raman cross sections $(d \sigma / d \Omega)$ in molecules are in general very difficult (and sometimes impossible) to obtain with normal (spontaneous) Raman spectroscopy. Despite their great importance in techniques such as surface-enhanced Raman scattering (SERS) [2], there is only a scant amount of experimental information on them [24]. The predominant reason for this is the simultaneous generation of fluorescence under resonance conditions in continuous-wave (CW) spectroscopy. For fluorescent dyes (which constitute a very important group of probes for SERS in general [2,4], and for single-molecule SERS in particu$\operatorname{lar}[9,11,12,25]$ the bare fluorescence cross section of the molecules $\left(d \sigma_{\text {fluo }} / d \Omega \sim 10^{-16} \mathrm{~cm}^{2} / \mathrm{sr}\right)$ typically outstrips the corresponding Raman ones in resonance ( $d \sigma_{\text {Raman }} / d \Omega \sim 10^{-24} \mathrm{~cm}^{2} / \mathrm{sr}$ ) by figures in the range of eight orders of magnitude. This is of course the primary reason why the field of single-molecule fluorescence [26, 27] was developed a lot earlier than its counterpart in Raman spectroscopy. Despite the fact that these cross sections refer to spectrally integrated quantities (i.e. integrated over all emission wavelengths) and that Raman signals are typically much "sharper" than fluorescence emission (by a factor of $\sim 10^{2}-10^{3}$ ), this is still not enough to compensate for the remaining five to six orders of magnitude in intensity between the two: Raman signals are basically "swamped" in the fluorescence background of the spectrum. There are exceptions to the rule though in CW spectroscopy. The most common of which comes from dyes with a poor quantum yield $(Q)$ for fluorescence emission $[2,27]$. One example of the latter is the molecule crystal violet (CV) [24], with a very low fluorescent quantum yield $Q \sim 5 \times 10^{-5}$ [28] due to internal non-radiative relaxation mechanisms [27] (the twisting of the arms of the molecule) in the excited state. Hence, in cases like CV it is indeed possible to measure the CW differential Raman cross section under resonance conditions, by comparing the signal of a known concentration of CV molecules in solution to a reference standard $[22,24]$ under identical experimental conditions. These cases are, nevertheless, more the exception than the rule for many important dyes. Paraphrasing a recent review article by Anne Myers-Kelley [29]: "Resonance Raman scattering ... largely places the experimentalist at nature's mercy. If the Raman spectrum is weak or extremely complicated, or sits on top of a strong fluorescence background, there is not much that can be done about it".

A notable exception to the deadlock imposed by the presence of underlying fluorescence has arisen in the last few years in the form of broadband femto-second stimulated Raman spectroscopy (FSRS); pursued mainly by Mathies and co-workers [30-34]. FSRS is insensitive to background spontaneous fluorescence [33], and its theory has also been well developed [32]. For many years, a historical predecessor of FSRS to unravel the Raman spectrum under resonance conditions has been the technique of Kerr-gated time-resolved resonance Raman spectroscopy [35]. The early time-resolved methods [36] were mainly based on the idea of measuring the Raman spectrum before the fluorescence arrives; i.e. in the window of a few nanoseconds (typically) defined by the lifetime of the excited state $[2,27]$. However, quantifying the differential cross section is still one step above the mere "extraction" of the Raman spectrum from the fluorescence background, for it implies a normalization with respect to a known standard that can be difficult to achieve in the time-resolved versions of the technique. The best evidence of how difficult it is to obtain reliable values of resonant Raman differential cross sections is perhaps the fact that the first published values in the literature for Rhodamine 6G (arguably one of the best known and most widely used dyes in laser spectroscopy) have been published for the first time in 2008 by Mathies and co-workers 
for a single probe excitation line at $532 \mathrm{~nm}$ (doubled Nd-YAG laser), using FSRS [30]. The progress made in FSRS in the last few years is truly impressive, but it still remains a technique that is practiced by a handful of groups around the world. To the best of our knowledge, there are a priori no obvious alternatives in plain CW Raman spectroscopy for the task.

On the theoretical front, quantitative predictions of resonant Raman cross-sections are also challenging since they require detailed modeling of excited state dynamics. It is only recently that reports of theoretical predictions have appeared. An example particularly relevant to this study is Ref. [37], where time-dependent density functional theory is used to predict resonant Raman cross-sections of Rhodamine 6G. Arguably, such theoretical predictions remain subject to potentially large errors.

In this chapter, we propose a new method to quantify experimentally resonant Raman differential cross sections by using the SERS effect and CW Raman spectroscopy. Like any method, it has intrinsic limitations, but in those cases where it can be applied it provides an easy alternative to time-resolved spectroscopy. It can produce experimental estimates for several excitation lines, and it can be used directly with CW-Raman systems that are most commonly available in many laboratories around the world (compared to the availability of time resolved systems). In the following, we explain the basics of the method and discuss its potential limitations. We then apply it specifically to obtain experimental estimations of $d \sigma / d \Omega$ for RH6G for seven different excitation lines across the visible range. 


\subsection{Principles of the method}

One important aspect of surface-enhanced spectroscopy is the interplay between radiative and non-radiative components of the enhancement factor, and how they affect (differently) Raman and fluorescence processes. In this chapter the basic concept, needed for the present problem, shall be briefly reviewed (with the aid of Fig. 12) while a more detailed explanation can be found in Section 1.3 and in the specialized literature $[2,28,38-40]$.

\subsubsection{Basic concept}

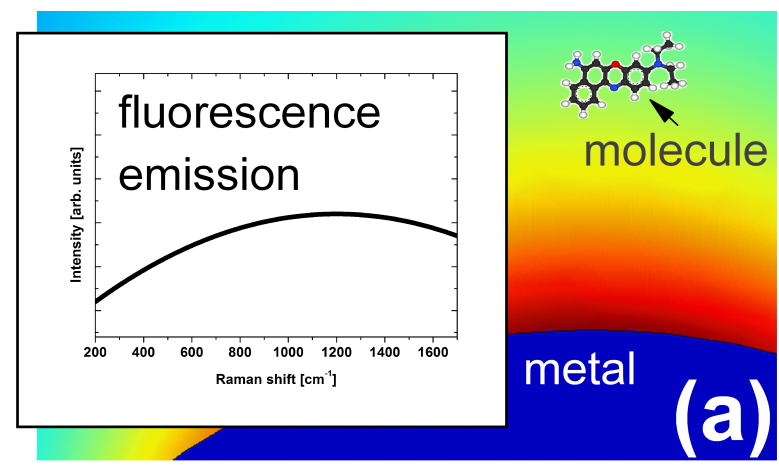

Fig. 12:

Schematic sequence illustrating the adsorption of a molecule on a metal surface and the concomitant quenching process:

(a) The emission far away from a metallic surface is dominated by fluorescence when the quantum yield of the dye is good.

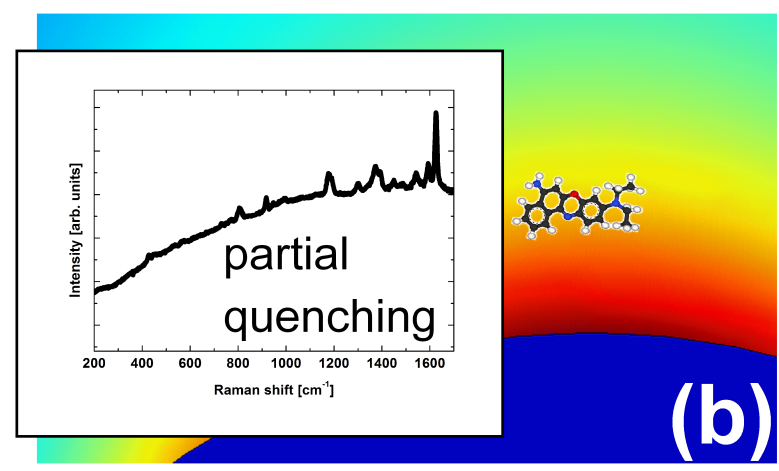

(b) As the dye approaches the surface, both Raman and fluorescence are enhanced; but while Raman always profits from radiative enhancement, the fluorescence emission is a compromise between what is gained in enhanced absorption and what is lost is nonradiative emission to the metal [2]. This is the well-known "quenching of fluorescence" effect seen in SERS.

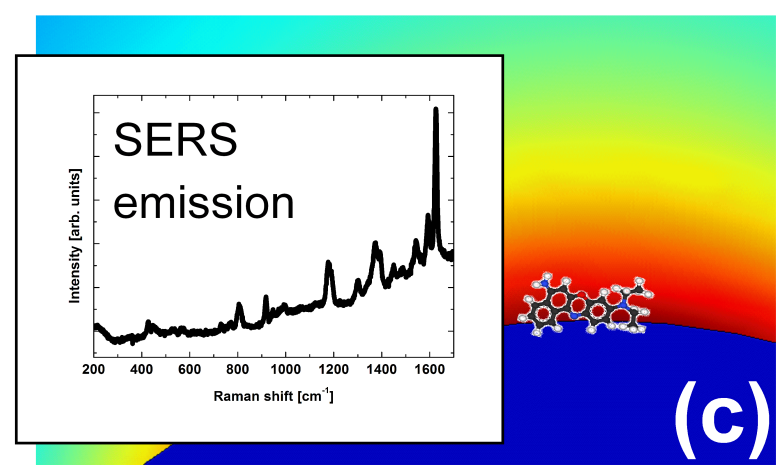

(c) In direct contact with the metal, the molecule's fluorescence is mostly quenched (with respect to SERS) by non-radiative processes. If we were able to compare two molecules (one with a known and another with an unknown $d \sigma / d \Omega$ ) on the same spot on the surface, we would have a (comparative) measure of Raman differential cross sections without interference from fluorescence.

In a nutshell, Raman profits from radiative enhancements in both the incoming (laser) and Stokesscattered photon, while fluorescence only profits from an absorption enhancement in the first 
step of the process. The emission in fluorescence is further limited by the competition between radiative and non-radiative decay from the excited state, the rate of which are modified by different mechanisms in close vicinity to a metal surface. As a result, for molecules adsorbed directly onto the metal, the Raman signal is typically enhanced by several orders of magnitude more than the fluorescence signal.

This phenomenology is often simplified by simply stating that "fluorescence is quenched while Raman is enhanced" as the molecule approaches the metal surface; this is illustrated schematically in Fig. 12(a-c) and treated in considerable more detail in the specialized literature $[2,28,38-41]$. In reality, fluorescence may still be enhanced when a molecule is directly adsorbed onto the metal $[2,41]$, but the fluorescence to Raman ratio is indeed strongly quenched.

Using this well-known phenomenology, we can qualitatively envisage the following method to quantify an unknown resonance Raman cross-section: we first take a reference molecule of which we know the bare Raman differential cross sections for some modes, and place that molecule in a specific place on a metal surface where it is subject to a Raman enhancement (i.e. we can measure its SERS spectrum). We then take a resonant molecule that we do not know the Raman differential cross sections of (because it was hidden by fluorescence), and place it in the exact same position as the previous one. Thanks to fluorescence quenching, we can again measure its SERS spectrum under identical experimental conditions as the reference. If both molecules are not intrinsically modified by the presence of the metal and both are subject to the same SERS enhancement, simple ratios of peak intensities yield the differential Raman cross sections of the second molecule.

\subsubsection{Potential limitations}

There are several problems with this simple-minded approach, but all of them admit some sort of a solution or a partial solution. We list a few of the most obvious here:

- The method implies that there is no substantial modification of the molecule as a result of its interaction with the surface; i.e. that there is no metal-molecule complex formed in the process. This aspect is closely related to the possibility of an additional "chemical enhancement" in SERS [42]. If there is a metal-molecule complex present, the differential cross section we measure is not that of the bare molecule but rather that of the molecule in the presence of the metal (which can have its resonance condition changed in the process). While this is a potential limitation, there is some reassurance that such a situation can in principle be detected by making measurements on several different dyes, over a large wavelength range, and ideally on two types of SERS substrates (e.g. silver and gold).

- The simple-minded approach as stated above implies that we are able to position the molecule and the reference molecule in exactly the same place. This is obviously unachievable, and it is exacerbated by the well-known drastic inhomogeneities of the enhancement factor in typical SERS substrates [10]. Instead we can rely not on single-molecule signals but on average properties of the enhancement factor for a given substrate [24]. For as long as the total concentrations of dyes are small enough to avoid mutual interference on the surface coverage, and large enough to ensure reproducibility of signals over the chosen integration time, we can take average values as representative. The underlying assumption here is that the 
average SERS EF (or analytical SERS EF [2, 24]) is similar for both dyes. We need for example to ensure that both dyes have the same adsorption properties on the metal surface (for example by choosing both dyes with a strong affinity to the metal surface).

- The idea also assumes that there will be no additional experimental limitation to obtain the SERS signals of both dyes. However, the unequal photobleaching rates for both dyes at a given excitation wavelength can make a difference in the comparison of relative intensities [43, 44]. Photobleaching problems can be avoided by working with the lowest possible power densities and their presence can be checked experimentally by performing measurements at two or more incident laser powers.

- In theory there is no limitation on how different the two differential Raman cross sections to be compared can be. In practice, however, it is best to be able to to see both signals within the same dynamic range of the detector without having to change the experimental conditions (increasing power, integration time, etc,...). This implies that we should try to use dyes with similar cross sections. If we are trying to measure a resonant dye, we then need to find another resonant dye with known cross section. Accordingly this second reference molecule cannot be anything but a resonant dye with a poor quantum yield. This enhances the importance for this application of resonant dyes with low (or negligible) quantum yields. Some standard dyes (like CV) fall into this category, but they can also be engineered if necessary by the addition of quenching moieties to a given chromophore. The choice of the "reference molecule" is an important part of the problem here, and it needs to be resolved on a case-bycase basis depending on the compatibility with the other molecule and the spectral range where we want the comparison to be made.

- SERS substrates tend to have problems with reproducibility. A rule of thumb is always that the smaller the enhancement the better the reproducibility of the signal [45]. Substrates that sustain high enhancements $\left(\sim 10^{9}\right)$ capable to see single molecules are typically the least reproducible. Part of this issue can be solved by working with low magnification objectives and long integration times to increase sampling range in space and time, respectively (this has the additional benefit of reducing photo-bleaching issues). But to completely avoid this problem, it is even better to have both dyes simultaneously in the sample. This is similar in some ways to the bi-analyte SERS technique [8], which was developed for single molecule detection. Here, the method is used at much higher analyte concentrations to compare $a v$ erage signals over the same enhancement factor distribution.

- Different surface selection rules $[13,46]$ for different molecules could pose a problem, because the electromagnetic SERS EF may vary from mode to mode and from molecule to molecule by a factor related to the orientation of the molecule on the surface. Except for extreme cases (where a peak disappears, for example) this should not contribute much except by a relatively small factor [46]. The cross-sections determined with this method are therefore subject to uncertainties in the surface-selection rules factor [2], which unless independently measured can be assumed to be of the order of a factor of $\sim 2$ [46].

Accordingly, the method proposed here is nothing but a bi-analyte SERS measurement, where the aim is not to use a contrast signal to detect single molecule cases but rather make a comparison of average signals over the same enhancement factor distribution. This is done for two different resonant molecules: one that we know the Raman differential cross sections of, and one that we do 
not. Note that we use SERS substrates here to profit not so much from their enhancing properties of the Raman signals (SERS) but mainly for their quenching properties of the fluorescence.

The main underlying assumption is that both molecules experience the same analytical SERS EF [24]. This implies in particular that they have the same adsorption efficiency during sample preparation, that they do not exhibit extreme surface selection rules effects, and no chemical modification or chemical enhancement. The approximate validity of these assumptions (say within a factor $\approx 2-3$ ) is, we believe, sufficiently common (see Ref. [24] for examples) but must nevertheless be assessed on a case-by-case basis. In particular, measurements on different types of SERS substrates or with different preparation procedures, with different pairs of analytes, and at different excitation wavelengths should all contribute to confirm, or disprove, the validity of these assumptions. In fact, as we shall see, there may in some cases be wavelengths for which the Raman cross-sections of the unknown dye can also be measured via standard methods (for example when it is in pre-resonance conditions). This then provides a further independent check of the validity of the proposed method.

With some limitations (summarized in the list above) a simple estimate of resonant Raman cross sections is feasible without resorting to time-resolved spectroscopy. The limitations are real, but there is no technique that is completely free of limitations in that sense. FSRS, for example, also faces limitations in terms of the photostability of the molecules under intense pulsed excitation and the actual Raman gain that can be obtained by stimulation. There will be examples where a quantitative determination of resonant differential cross sections with FSRS will not be possible. With these provisos in mind, when the technique proposed here is applicable, it does provide a very simple estimation of resonant differential Raman cross sections.

Table 2.1: CV cross-sections for the $1620 \mathrm{~cm}^{-1}$ mode along with experimental conditions (CV concentration $c$, integration time $t$, and incident power $P$ ), correction factors $K_{\mathrm{abs}}$ (accounting for absorption losses in the liquid solution of the incident laser-light (on its way from the objective to its focal point) and the scattered Raman-light (on its way from the focal point back to the objective)) and $K_{\text {sys. }}$ (accounting for the the fact that for each excitation wavelength the detection efficiency of the system at the wavelength associated with 2B2MP's $516 \mathrm{~cm}^{-1}$ Raman peak differes from the detection efficiency of the system at the wavelength associated with CV's $1620 \mathrm{~cm}^{-1}$ Raman peak) and measured $\mathrm{CV}$ depolarization ratios $\rho=I_{\perp} / I_{\|}$used for determining it.

\begin{tabular}{l|l|l|l|l|l|l|l}
$\lambda_{\text {ex }}[\mathrm{nm}]$ & 458 & 488 & 514 & 532 & 568 & 633 & 647 \\
\hline \hline$c[\mu \mathrm{M}]$ & 50 & 10 & 10 & 10 & 10 & 100 & 100 \\
$t[\mathrm{sec}]$ & 25 & 100 & 200 & 100 & 30 & 60 & 100 \\
$P[\mathrm{~mW}]$ & 4.0 & 2.0 & 2.0 & 2.0 & 2.0 & 2.0 & 2.0 \\
\hline$K_{\text {sys. }}$ & 2.74 & 1.37 & 1.07 & 1.02 & 0.88 & 0.69 & 0.62 \\
$K_{\text {abs. }}$ & 2.44 & 1.63 & 2.13 & 2.84 & 1.97 & 2.51 & 1.43 \\
$\rho$ & 0.77 & 0.29 & 0.31 & 0.45 & 0.36 & 0.35 & 0.28 \\
\hline $\mathrm{d}_{\Omega} \sigma\left[10^{-26} \frac{\mathrm{cm}^{2}}{\mathrm{sr}}\right]$ & 4.6 & 9.7 & 14.4 & 28.5 & 67.5 & 5.1 & 1.7
\end{tabular}




\subsection{Experimental}

As an example of its applicability, in the next two sections ('Experimental" and "Data evaluation and results") data shall be provided and evaluated for the estimation of resonant Raman differential cross sections $\mathrm{d}_{\Omega} \sigma^{\mathrm{RH} 6 \mathrm{G}}(\lambda)$ for Rhodamine $6 \mathrm{G}$ (RH6G) at seven different excitation wavelengths in the visible by a direct comparison with CV. To this end we proceed as follows:

1. First the bare differential Raman cross-sections $\mathrm{d}_{\Omega} \sigma^{\mathrm{CV}}(\lambda)$ of $\mathrm{CV}$ are obtained for different excitation wavelengths $\lambda$.

2. Then, ratios $R_{i}(\lambda)$ of SERS signals of these two dyes in four different types of SERS substrates (indexed with $i$ ) are measured.

The desired cross-section $\mathrm{d}_{\Omega} \sigma^{\mathrm{RH} 6 \mathrm{G}}(\lambda)$ of Rhodamine6G is obtained, by multiplying the average ratio $\langle R(\lambda)\rangle$ with the measured reference cross-section $\mathrm{d}_{\Omega} \sigma^{\mathrm{CV}}(\lambda)$.

\subsubsection{Determination of the reference cross-section $\mathrm{d}_{\Omega} \sigma^{\mathrm{CV}}(\lambda)$}

Raman spectra of Crystal Violet (CV) were taken with a triple-subtractive Jobin-Yvon Raman microscope equipped with a $\mathrm{N}_{2}$-cooled CCD detector. Different excitations lines in the blue-red region $(458,488,514,532,568,633$, and $647 \mathrm{~nm})$ were obtained from either a HeNe or tunable $\mathrm{Ar}^{+}$$\mathrm{Kr}^{+}$laser. The triple-subtractive spectrometer is ideal for resonance studies, for it does not rely on the use of notch filters.

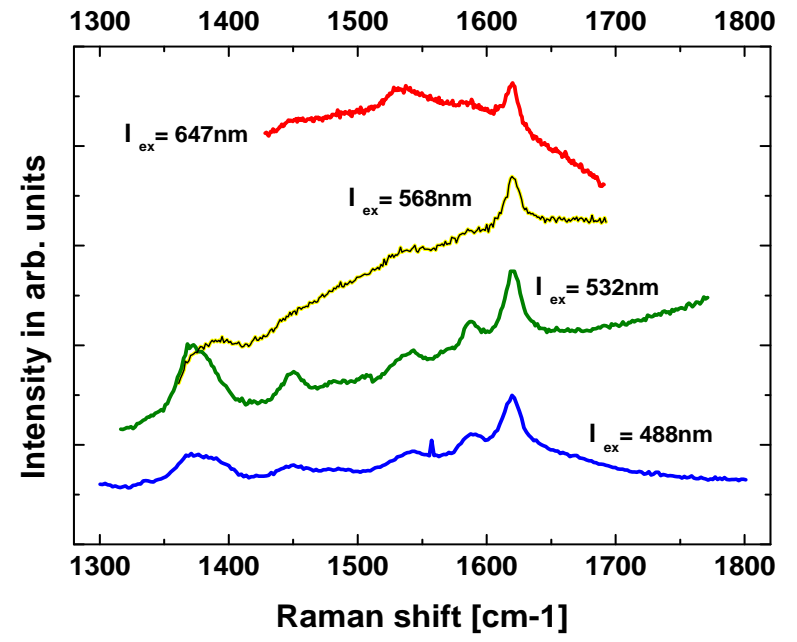

Fig. 13: Examples of bare $C V$ Raman spectra taken at 4 different excitation wavelengths $\lambda_{\text {ex }}$. All of these spectra show the distinct peak at $1620 \mathrm{~cm}^{-1}$ above a residual fluorescence background.
In order to determine CV's resonant Raman cross-section, we focused on its $1620 \mathrm{~cm}^{-1}$ mode and compared it with a standard: 2-bromo-2-methylpropane (2B2MP) (thoroughly characterized in Ref. [24]). We use, in particular, the $516 \mathrm{~cm}^{-1}$ Raman mode of 2B2MP which has a differential cross section of $5.4 \times 10^{-30} \mathrm{~cm}^{2} / \mathrm{sr}$ at $633 \mathrm{~nm}$ and can be corrected for other excitation wavelengths $\lambda_{\text {ex }}$ by a factor of approximately $\left(633 / \lambda_{\mathrm{ex}}\right)^{4}$ where $\lambda_{\text {ex }}$ is in nm (since it is far from resonance across the visible).

By knowing the concentration of CV in a reference solution and the density of 2B2MP, a direct intensity ratio provides the differential cross sections for the former, even without characterizing the scattering volume [24]. Examples of Raman spectra are shown in Fig. 13 while the experimental conditions used are summarized in Table 2.1.

While photobleaching is always a potential danger to the measurements' accuracy, it was accounted for by using low power densities (i.e. a low magnification x20 objective). Furthermore 
the absence of photobleaching was affirmed by checking the signals' linear behavior with varying laser powers. However, there are three additional complications to this standard measurement that need to be accounted for:

Firstly, the solution is not transparent, and its absorption of both the laser and Raman light must be taken into account. To correct this, we measured the dye's absorbance and calculated the loss in incident power as well as the loss in scattered light taking into account the shift in wavelength. This leads to a correction factor $K_{\text {abs. }}$ (for each excitation wavelength), which is listed in Table 2.1 (the focusing distance of the objective is $3.3 \mathrm{~mm}$ ).

Secondly, the detection efficiency of our spectrometer is polarization dependent. In consequence all Raman spectra were taken with a polarizer for detection parallel to the incident polarization. We therefore measure the ratio of $d \sigma_{\|} / d \Omega$ rather than that of $d \sigma / d \Omega$. This can be corrected using the expression:

$$
\frac{\mathrm{d} \sigma}{\mathrm{d} \Omega}=\frac{\mathrm{d} \sigma_{\|}}{\mathrm{d} \Omega}+\frac{\mathrm{d} \sigma_{\perp}}{\mathrm{d} \Omega} \stackrel{\text { eq.1.6 }}{=}(1+\rho) \frac{\mathrm{d} \sigma_{\|}}{\mathrm{d} \Omega}
$$

Here $\rho$ is the depolarization ratio of the mode [2]. To apply this correction, we have used the value $\rho=0.18$ for 2B2MP [24] and have measured $\rho$ for CV at all excitation wavelengths, again listed in Table 2.1.

Thirdly, for a given excitation wavelength $\lambda_{\text {ex }}$ the detection efficiency of the system varies from the reference mode of 2B2MP (@516 $\mathrm{cm}^{-1}$ ) to that of CV (@ $1620 \mathrm{~cm}^{-1}$ ). To correct this we measured the response of our system (depicted in Fig. 14) by comparing the signal with the black body spectrum of a calibrated lamp of known effective temperature $(T=2700 \mathrm{~K})$.

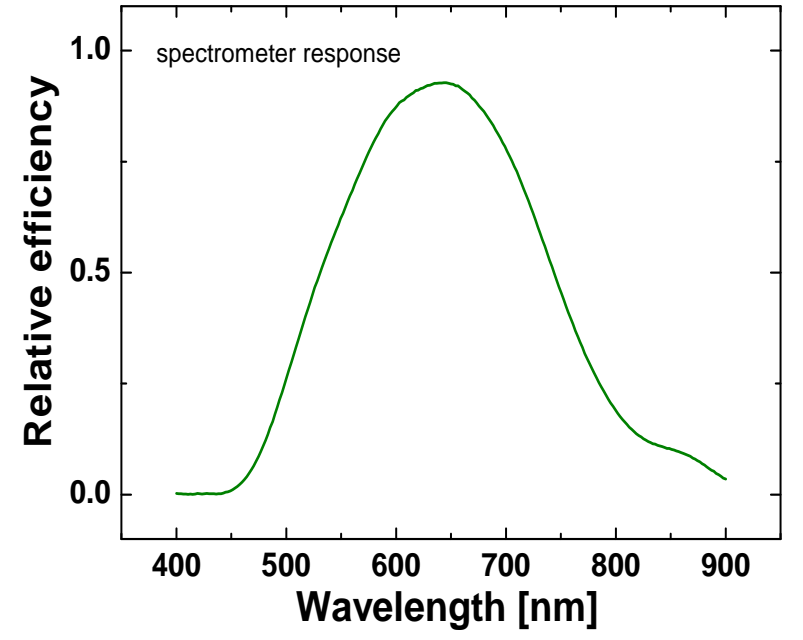

Fig. 14: Response (relative efficiency) of our spectrometer at different wavelengths measured with a lamp of which the emission spectrum is known.
From this curve the correction factor $K_{\text {sys. }}$ is extracted which is again listed in Table 2.1. $\mathrm{CV}$ cross-section values in the green-yellow region are not affected much by the different response, but in the blue-purple region the correction can be important.

Comparing a mode, for example, at a Raman shift of $516 \mathrm{~cm}^{-1}$ (2B2MP) with one at $1620 \mathrm{~cm}^{-1}$ (CV) for $458 \mathrm{~nm}$ laser excitation (which places the modes in absolute wavelengths at 469 and $495.4 \mathrm{~nm}$, respectively) overestimates the differential cross section of CV by a factor of $\sim 3.7$, unless the correction for the response of the system is included. All values in Fig. 14 are corrected by the response of the system. The correction factors $\mathrm{K}_{\text {sys. }}$ (see Table 2.1) are extracted from this curve.

The results for $\mathrm{d}_{\Omega} \sigma^{\mathrm{CV}}(\lambda)$ are summarized in Table 2.1 along with measurement details and the correction factors. It is interesting to note the variations of $\rho$ as a function of excitation wavelength, a clear indication of mode symmetry changes induced by resonance [47]. 


\subsubsection{Determination of the relative intensity ratios $R_{i}(\lambda)$}

The ratios of SERS signals of RH6G $\left(1510 \mathrm{~cm}^{-1}\right.$ peak $)$ and the reference molecule CV $\left(1620 \mathrm{~cm}^{-1}\right.$ peak) were taken on four different types of substrates (Ag colloids in solution, silver films, Au films, and Klarite substrates, see Section 1.5.1) and for 7 excitation wavelengths.
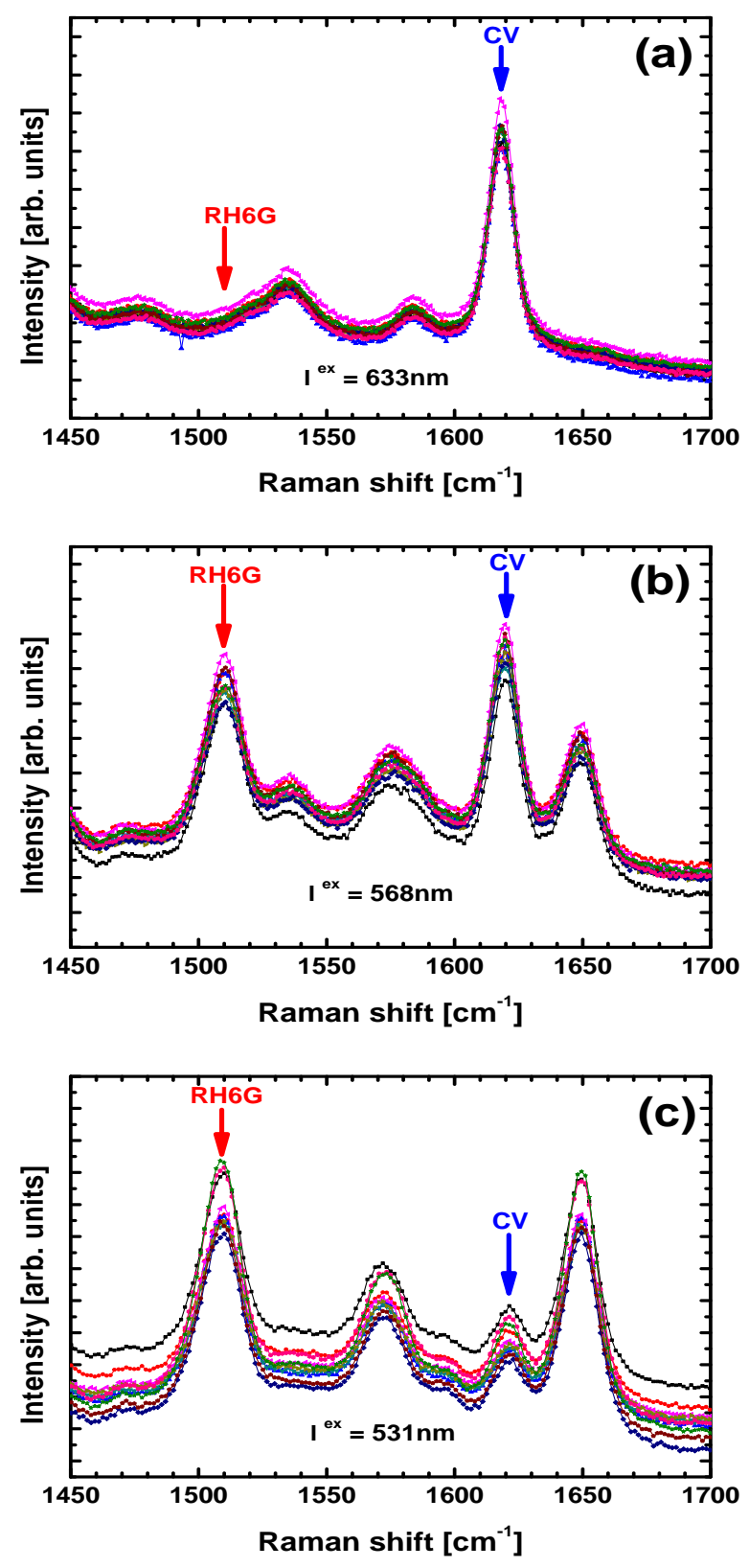

Fig. 15 : Examples of data for a sample with a concentration ratio $R H 6 G: C V=1: 1 \quad(10 \mathrm{nM}$ in total), measured at different excitation wavelengths: (a) $\lambda_{\mathrm{ex}}=633 \mathrm{~nm}$, (b) $568 \mathrm{~nm}$ and (c) $531 \mathrm{~nm}$.
In principle, the nature of the SERS substrate should not influence the results for as long as: $(i)$ the substrate does not interfere with the dyes, and $(i i)$ there is enough signal to observe both dyes (the reference and the probe). It is a good practice however to compare the ratios of Raman signals under SERS conditions on several different substrates. This is done to ensure that there was nothing extremely peculiar about one particular choice of substrate and that similar results can be obtained irrespective of the exact nature of it. If this is the case, we can have more confidence that what we are measuring is an intrinsic property of the probe, and not a property of the probe in combination with the substrate. If the substrate is completely insensitive to the ratios of cross sections between an unknown probe and a reference, it should not even matter whether we use silver or gold (the two most widely used SERS substrates) as enhancing/quenching materials.

The first series of samples are prepared in solution with Ag Lee \& Meisel colloids [19] at $15 \mathrm{mM} \mathrm{KCl} \mathrm{[48].} \mathrm{Samples} \mathrm{were} \mathrm{pre-}$ pared to a total dye concentration of $10 \mathrm{nM}$ (CV+RH6G) by successive dilutions from reference samples at $100 \mu \mathrm{M}$. To avoid dilution errors in this process, we obtained the final concentration in three steps. Initially we go from $100 \mu \mathrm{M}$ to $1 \mu \mathrm{M}$, where an absorbance measurement is carried out to ensure proper dilution. At this point the dyes are mixed in a 1:1 (or 10:1) ratio and further diluted by a factor of 50 in the colloid, which is then aggregated with $\mathrm{KCl}$. It is worth pointing out at this point that the accurate preparation of low dye concentrations is a challenging aspect of this experiment and should always be done with care. 
As a way of example, with a fluorometer we were able to check concentrations down to $\sim 1 \mathrm{nM}$ and observed deviations from the desired value of up to a factor of $\sim 3$ in many instances. With the final procedure we followed (which included carefully electrostatically discharging the equipment we used) we were able to follow a reliable dilution allowing us to obtain the desired concentrations with an accuracy of about $\sim 10 \%$ in the final concentration.

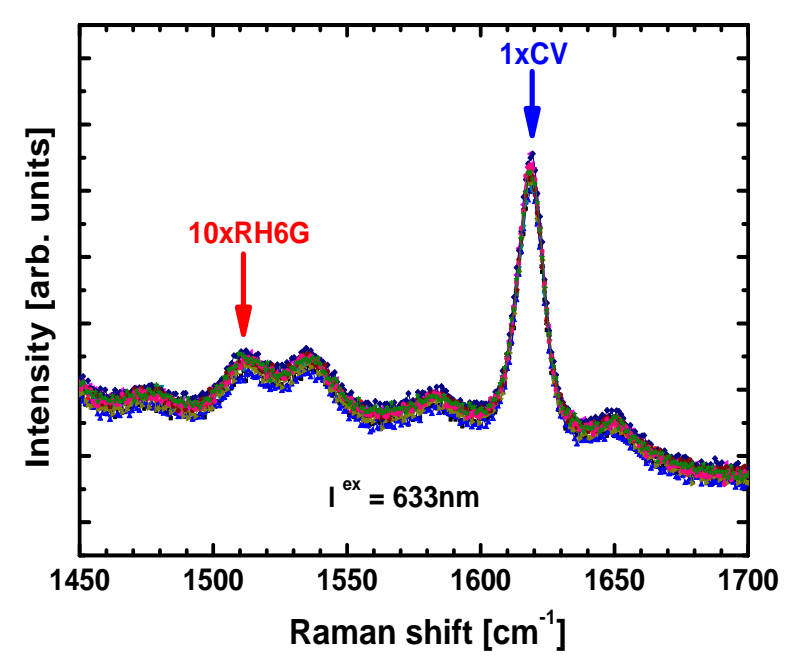

Fig. 16: Sample with RH6G:CV = 10:1, measured at excitation wavelength $\lambda_{\mathrm{ex}}=633 \mathrm{~nm}$. The difference in cross sections is partially compensated by an increase in the relative concentration of the two dyes.
Figures 15 and 16 show a few examples of measurements done in the Ag Lee \& Meisel colloids at three different excitation wavelengths. We normally take several spectra (as shown in the figure) over time (with 30 to $200 \mathrm{sec}$ integration time) to obtain an average on which the analysis is performed. This is done to ensure that the average signal was not affected by an unusually large fluctuation of the signal (which can happen sometimes with large clusters in colloidal suspensions).

RH6G goes from dominating the spectrum over CV in the green $(532 \mathrm{~nm})$ to almost disappearing from it in the red $(633 \mathrm{~nm})$. The latter suggests that its cross-section in the red is at least an order of magnitude smaller than CV. In order to verify this, a new sample was prepared where RH6G and CV were diluted as before, but this time with a relative

concentration of 10:1 (10 $\mathrm{nM}$ in total). Figure 16 shows complementary data (equivalent to the data in Fig. 15) for this additional sample. For the seven wavelengths used for this experiment laser powers between 100 and $1000 \mu \mathrm{W}$ with the $\times 20$ immersion objective were chosen. No detectable effect of photobleaching was observed at this laser power level.

In addition to colloid measurements, we check the relative ratios of SERS signals for the dyes deposited at $\sim 100 \mathrm{nM}$ concentration on flat silver and gold films, and commercial Klarite substrates (made out of gold). Silver $(30 \mathrm{~nm})$ and gold $(200 \mathrm{~nm})$ films were deposited on glass slides using a conventional evaporator equipped with a calibrated thickness monitor. Klarite substrates were used as received without further preparation. The dyes (and dye mixtures) were drop cast on the films and/or Klarite substrate and dried under a mild heat. Raman spectra were taken in this case with a $\times 10$ objective (to improve spatial averaging and avoid photobleaching as much as possible) in air, by using again the T64000 Jobin-Yvon triple spectrometer and integration times of $\sim 20-60$ sec. We made averages over at least 20 different points on the sample to ensure the signal was representative of the average over the substrate. The data on gold substrates tends to be weaker than that on silver films, and it is likely that part of the enhancement seen on silver and gold films comes from surface roughness. For gold substrates we could only obtain spectra at three of the six laser wavelengths we used for silver; this is because the signal is too weak and cannot be quantified in the other cases.

For the analysis, we concentrate only on two modes: $1510 \mathrm{~cm}^{-1}$ of RH6G and $1620 \mathrm{~cm}^{-1}$ of CV simply because they do not overlap too much with other modes and can be easily measured within 
the same spectral window of the CCD. In order to obtain the cross-section ratio

$$
R\left(\lambda_{\mathrm{ex}}\right):=\frac{\mathrm{d}_{\Omega} \sigma_{\mathrm{RH} 6 \mathrm{G}}^{1510}\left(\lambda_{\mathrm{ex}}\right)}{\mathrm{d}_{\Omega} \sigma_{\mathrm{CV}}^{1620}\left(\lambda_{\mathrm{ex}}\right)}
$$

of the differential cross-section ratios for the different excitation wavelengths $\lambda_{\mathrm{ex}}$, we assumed that both RH6G and CV experience the same enhancement factor in a bi-analyte SERS sample. In consequence, it is obvious that $R$ is equal to the ratio of the integrated peak intensities $\Sigma$ of the respective dye in the mixed sample:

$$
R=\frac{\sum_{\text {bi-analyte }}^{1510}}{\sum_{\text {bi-analyte }}^{1620}}
$$

However extracting those peak intensities from the bi-analyte SERS spectra is not trivial. The problem we are facing is possible overlap of peaks belonging to the different dyes which makes the fitting in the bi-analyte spectrum prone to error. A way to avoid such errors is schematically illustrated in Fig. 17 and explained in more detail in the following.
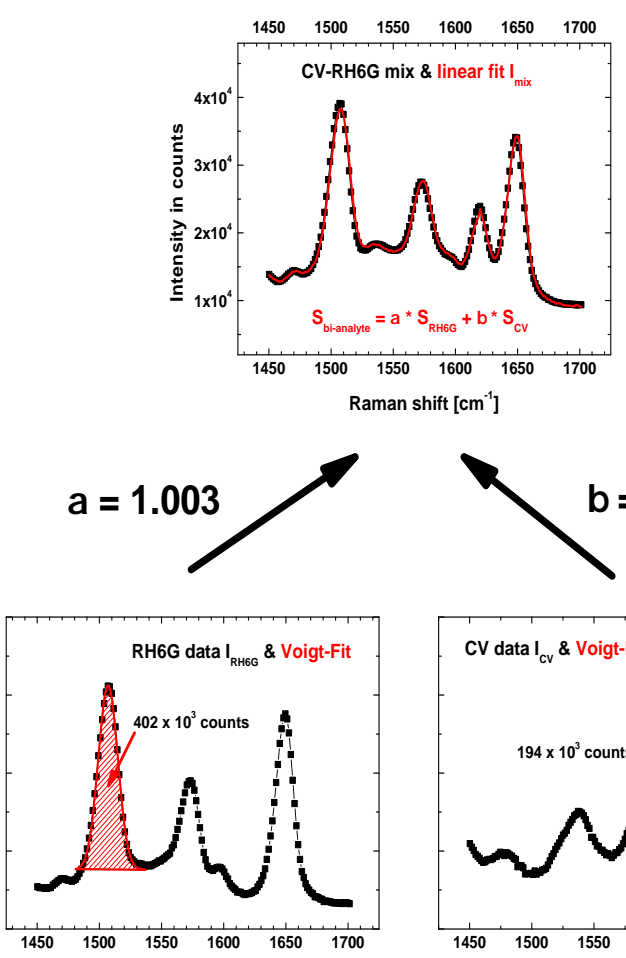
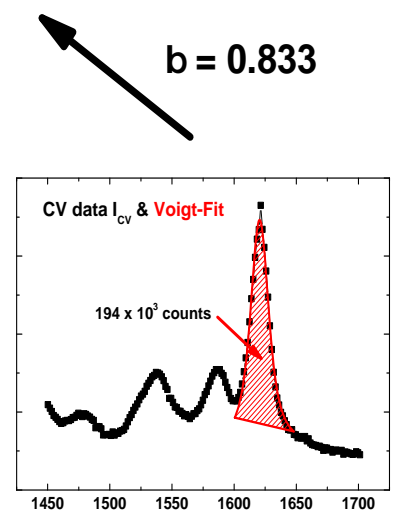

Fig. 17:

Schematic illustrating the method to extract the ratio $R$ of relative peak intensities from a bi-analyte spectrum (top spectrum). First the peak intensities $\Sigma_{\mathrm{RH} G \mathrm{G}}^{1510}$ and $\Sigma_{\mathrm{CV}}^{1620}$ of a pure $R H 6 G\left(S_{\mathrm{RH} 6 \mathrm{G}}\right.$, bottom left) and a pure CV SERS spectrum $\left(S_{\mathrm{CV}}\right.$, bottom right) are determined. In a second step a linear least-square decomposition of the mixed spectrum $S_{\mathrm{bi}-\text { analyte }}$ into its components $S_{\mathrm{RH} 6 \mathrm{G}}$ and $S_{\mathrm{CV}}$ (and a quadratic background) yields factors $\alpha$ and $\beta$. Note the accuracy of this fit. The ratio is then simply obtained from eq. 2.3 .

For each excitation wavelength $\lambda_{\mathrm{ex}}$, the fits of the peaks were first performed on a pure RH6G and on a pure CV SERS spectrum $\left(S_{\mathrm{RH} 6 \mathrm{G}}\right.$ and $S_{\mathrm{CV}}$ respectively). This provides us with peak Intensities $\Sigma_{\mathrm{RHGG}}^{1510}$ and $\Sigma_{\mathrm{CV}}^{1620}$ for each dye in two reference samples not affected by peak overlap. Those pure SERS spectra were then taken as a basis for a linear least-square decomposition of the bi-analyte data $\left(S_{\mathrm{bi}-\text { analyte}}\right.$ ) yielding factors $\alpha$ and $\beta$ that fulfill the following equation:

$$
S_{\text {bi-analyte }}=\alpha S_{\mathrm{RH} 6 \mathrm{G}}+\beta S_{\mathrm{CV}}+\text { background }
$$


The background (which consists of instrumental background and fluorescence) was taken as a quadratic function of the wavelength (which preserves the linearity of the decomposition). This linear least-square decomposition of a mixed SERS spectrum is extremely accurate since it is not affected by non-symmetric peak shapes or peak overlap. Knowing the background and the coefficients $\alpha$ and $\beta$, the ratio $R$ is then easily obtained from the relation $R=\alpha \Sigma_{\mathrm{RHGG}}^{1510} / \beta \Sigma_{\mathrm{CV}}^{1620}$ and leads to the SERS intensity ratios shown for all the different substrates in Fig. 18.

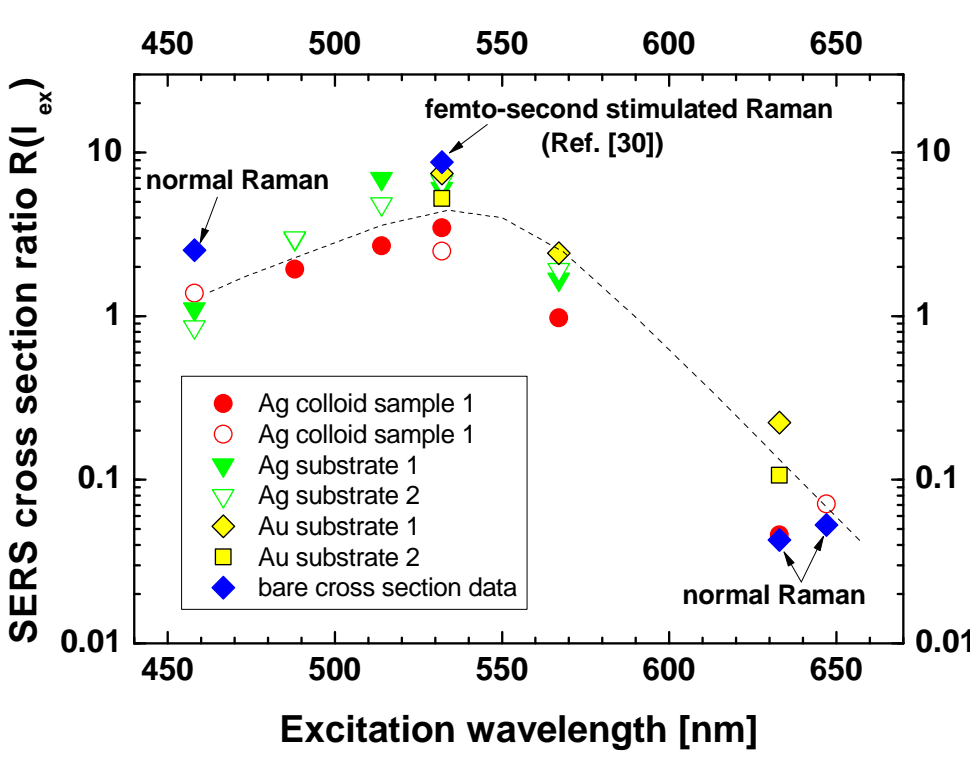

Fig. 18:

Ratios $R\left(\lambda_{\mathrm{ex}}\right)$ of SERS intensities of the RH6G and $C V$ peak $\left(1510 \mathrm{~cm}^{-1}\right.$ and $1650 \mathrm{~cm}^{-1} \mathrm{re}-$ spectively) as measured in the bianalyte experiment on different substrates. The blue, plain diamonds are reference points and correspond to ratios of bare cross sections $\sigma_{\mathrm{RH} 6 \mathrm{G}} / \sigma_{\mathrm{CV}}$ (see Table 2.2 and text for further explanation). The dashed line is only a guide to the eye. The differential cross sections of RH6G $d \sigma_{\mathrm{RH} 6 \mathrm{G}, \mathrm{SERS}} / d \Omega$ is then simply obtained with eq. 2.2 (see Table 2.2).

These SERS inferred ratios are shown, where possible, together with ratios of bare cross section measurements done with normal (CW) Raman spectroscopy. The latter are measured in regions outside the main absorption of RH6G (where we can see the Raman signal above the fluorescence background). One additional value is inferred from the bare cross section of RH6G measured by FSRS in Ref. [30] at $\lambda_{\mathrm{ex}}=532 \mathrm{~nm}$ and our own determination of the resonant cross section of CV at that wavelength.

To a very good approximation, a consistent picture is obtained for the ratios of cross sections across different substrates and different excitations. From these ratios and the bare $d \sigma / d \Omega$ 's of $\mathrm{CV}$, we can obtain estimates for the differential cross sections of the $1510 \mathrm{~cm}^{-1}$ mode of RH6G at different excitation wavelengths. The obtained differential cross-sections $d \sigma / d \Omega$ for RH6G and CV are summarized simultaneously in Table 2.2 and in Fig. 19. Even though listed with two significant figures the values for $\sigma_{\text {RHGG,SERS }}$ should be taken with caution since there is some scatter in the measurement. A rough estimate for their correctness is about a factor of 2. 
Table 2.2: Differential Raman cross-sections $\mathrm{d}_{\Omega} \sigma$ in units $\times 10^{-26} \mathrm{~cm}^{2} / \mathrm{sr}$ for CV $\left(1620 \mathrm{~cm}^{-1}\right.$ peak $)$ and RH6G $\left(1510 \mathrm{~cm}^{-1}\right.$ peak). The values $\sigma_{\text {RHGG,SERS }}$ were obtained by multiplying $\sigma_{\mathrm{CV}}\left(\lambda_{\mathrm{ex}}\right)$ with the ratio factor $R\left(\lambda_{\text {ex }}\right)$ obtained from the bi-analyte SERS spectra.

\begin{tabular}{l|l|l|l|l|l|l|l}
$\lambda_{\mathrm{ex}}[\mathrm{nm}]$ & 458 & 488 & 514 & 532 & 568 & 633 & 647 \\
\hline \hline$\sigma_{\mathrm{CV}}$ & 4.6 & 9.7 & 14 & 29 & 68 & 5.1 & 1.7 \\
\hline$\sigma_{\text {RHGG }}$ & 8.9 & & & $2.3 \times 10^{2}[30]$ & & 0.22 & 0.095 \\
\hline$\sigma_{\text {RHGG,SERS }}$ & 5.2 & 26 & 70 & $1.5 \times 10^{2}$ & $1.2 \times 10^{2}$ & 0.64 & 0.12
\end{tabular}

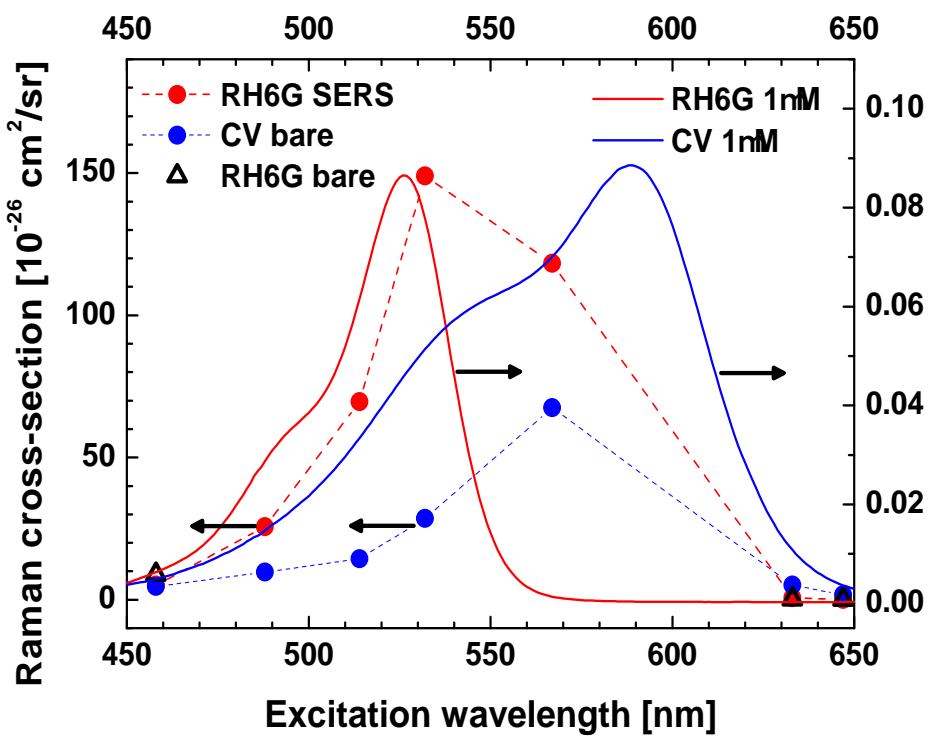

Fig. 19:

Differential Raman cross sections $d \sigma / d \Omega$ in units of $\times 10^{-26} \mathrm{~cm}^{2} / \mathrm{sr}$ for the $1620 \mathrm{~cm}^{-1}$ mode $C V$ and the $1510 \mathrm{~cm}^{-1}$ mode of RH6G at seven different excitation wavelengths $(458,488,514,532,568$, 633 and $647 \mathrm{~nm})$. The data is presented together with the absorption spectra of both dyes. RH6G displays a much stronger preresonance enhancement of the Raman cross section compared to $C V$ which seems to follow the absorption spectrum more closely. 


\subsection{Discussion and conclusion}

With several provisos in mind (that need to be evaluated on a case-by-case basis) we have shown here that it is indeed possible to obtain estimates of resonant differential Raman cross sections of dyes with SERS. Using the "quenching" properties of metals for the fluorescence emission of resonantly excited dyes, we can obtain estimates by comparison with a standard affected by the same enhancement distribution. We provide in Fig. 19 and Table 2.2 what we believe is the most complete set of values of resonant Raman differential cross sections for RH6G at seven different excitation wavelengths. Our values at $532 \mathrm{~nm}$ compare well with that obtained by the more sophisticated (and naturally more complicated) technique of femto-second stimulated Raman spectroscopy [30]. By the same token, we provide the wavelength-dependent resonant differential cross sections of CV in Tab. 2.2 and Fig. 19 (and the corresponding depolarization ratios in Tab.2.1), which were not reported before in the literature, to the best of our knowledge.

Note that RH6G seems to show a much stronger pre-resonance enhancement of the Raman cross section compared to $\mathrm{CV}$ in which the Raman resonant profile copies rather faithfully the dispersion of the absorption. In addition, the fact that the ratios of cross sections for the bare molecules at 647,633, and $458 \mathrm{~nm}$ (blue data points in Fig. 18) show some reasonable agreement with what happens for the same ratio on different substrates (within the limited natural experimental accuracy of these studies) goes some way towards addressing one of the issues raised earlier; i.e. the fact that the analytical SERS EF are the same for both dyes under a given set of experimental conditions. This agreement for example automatically excludes potential problems of different adsorption properties. We believe these results indicate that the enhancement is mainly electromagnetic in nature here (unless both molecules happen to have accidentally the same chemical enhancement, an unlikely situation). Similar evidence for CV and RH6G has been found before [24].

In more general terms, our paper here shows a quantitative application of SERS, that allows the experimental estimation of an extremely difficult intrinsic physical property of molecules (the resonant differential Raman cross section). Arguably, one of the main point of criticism of SERS over the years has been its inability to produce reliable quantitative results; a trend that is being slowly reverted in recent times by the development of better and more reproducible SERS substrates. Our method here "circumvents" the variabilities in the exact enhancement factor by maximizing as much as possible spatial and time averaging of the signals and by using two dyes simultaneously. It also highlights one of the less commonly utilized benefits of SERS (as opposed to the SERS enhancements), namely its ability to quench fluorescence with respect to Raman. 


\section{Chapter 3}

\section{Surface selection rules in SERS}

The work discussed in this chapter was published in "Chemical Communications", 2011 [49].

\subsection{Basic idea}

The Raman signal of a molecule attached to a metal surface depends on its orientation with respect to the surface, an effect that is formally described by SSR. The theory of the latter is well understood but has been confirmed so far only for relatively complex systems relying on a number of assumptions and simplifications. In this chapter we make the most direct demonstration of their validity by looking at molecules on a flat surface and are thereby able to deduce their orientation.
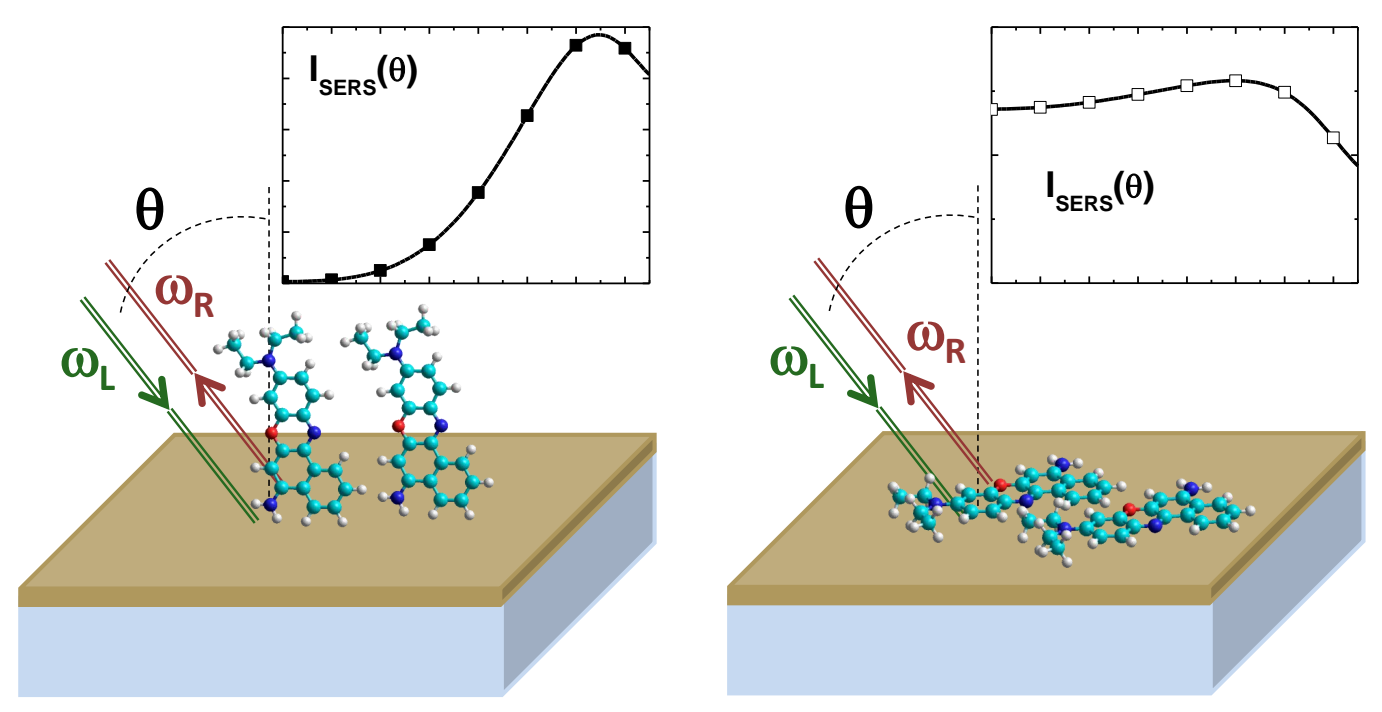

Fig. 20 : Illustration of one of the central ideas in this chapter: exploiting Surface Selection Rules in order to obtain the molecular orientation of a dye deposited on a Au surface. 


\subsection{Context: historical background and relevance}

SERS is a unique characterization tool for molecules on metal surfaces. The determination of molecular orientation with SERS has been a longstanding goal [50, 51]. For a fixed orientation on a metallic surface, the exact SERS intensity of each Raman mode depends on its tensor symmetry with respect to the surface, i.e. its orientation. Two main difficulties arise: $(i)$ the knowledge of the Raman tensors of different modes, and $(i i)$ the knowledge of the local field polarization at the molecule, and its connection with the SERS EF. The Raman tensors can be obtained for small molecules by symmetry analysis or density functional theory (DFT). However, some may be modified upon adsorption (for instance in chemisorbed molecules, which are precisely the most likely to have a fixed orientation). The theoretical basis for the local field polarization was laid out by Moskovits [13], who introduced the concept of SSRs and studied the case of planar metallic surfaces in detail. The concepts were also extended to the case of a dimer of two metallic spheres [46], a typical situation for SERS. SSRs also play an important role in interpreting some experiments probing the SERS chemical enhancement [52].

Most experimental studies of SSRs have been performed in colloidal solutions [53, 54], where signal strength is not an issue but interpretations of molecular orientation are troublesome. They are purely based on the qualitative assumption that the local field polarization is primarily $\perp$ to the metallic surface. Hence, modes with a strong Raman tensor component normal to the surface are more enhanced than those with weak (or nil) normal components. By comparing the SERS EF of the various modes, and relating these to their bare Raman tensors, the molecular orientation can be partly inferred (in particular, whether the molecule sits flat or upright on the surface). Assuming the molecule has a well-defined adsorption orientation with respect to the surface, a number of conditions are required for such an approach to work: $(i)$ The local field polarization must be predominantly $\perp$ to the surface: in particular at positions with the highest enhancements (there is no guarantee for this in complex substrates). ( $i$ i) The molecule must have several Raman modes with very different symmetries. The $\mathrm{CH}$-stretch of small aromatic molecules can, for example, satisfy these conditions [54]. However, this typically prevents the study of resonant molecules like dyes (very common in SERS), since all the tensors in resonant Raman conditions have typically the same symmetry (imposed by the resonant electronic transition). (iii) The variations of the SERS EF across modes must be large enough to be distinguished from other effects. Among them, the unavoidable presence of plasmon resonance dispersion which introduces a Raman-shift dependence of the enhancements $[47,55,56]$. (iv) The SERS EF of each mode must be measurable since the important parameter is the relative SERS EFs of the various modes, not their relative SERS intensities. The need to know the non-SERS spectrum for this step again prevents the use of resonant molecules (like dyes).

These limitations provide the motivation to revisit the possibility of measuring SSRs on flat metallic surfaces, as presented in this chapter. Provided there is no roughness, flat surfaces bestow the ultimate uniformity for SERS. Moreover, by changing the incident angle $\theta$ (Fig. 21) and polarization, we can modify the various components of the local field on the surface, thereby providing much richer information about tensor components. This lifts most of the limitations mentioned above, and leaves us with the "easier" problem of relating the Raman tensor to molecular orientation. The main disadvantage is that flat metallic surfaces have predicted SERS EF in the range $\sim 0-3$. Thus SERS experiments become challenging with the concomitant low signals. Despite this, we demonstrate SSRs on a flat Au film (no roughness), i.e. in the spirit of the original proposal 
[13] and as schematically illustrated in Fig. 21.

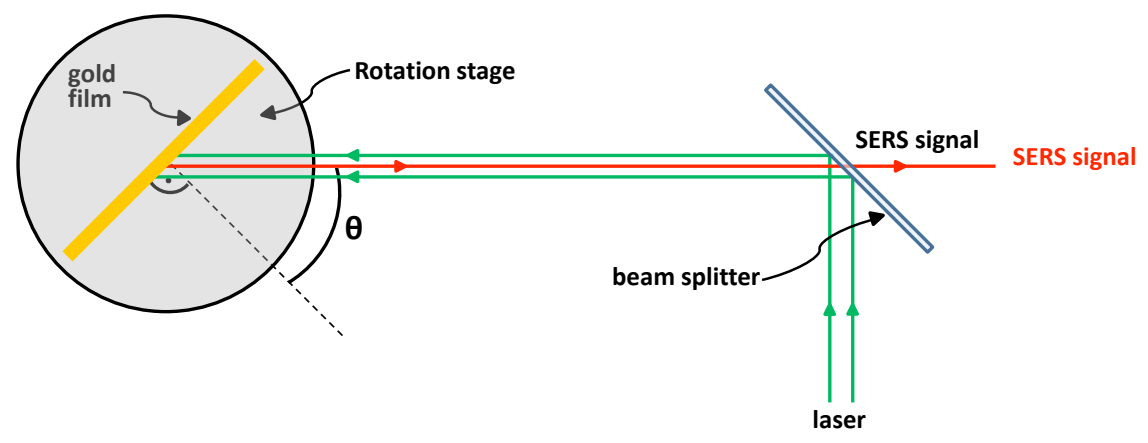

Fig. 21 : Schematic illustration of experimental setup used to obtain the data shown in this chapter.

To compensate for low SERS EFs, we use a resonant analyte: NB at $633 \mathrm{~nm}$ excitation. The molecular orientation is inferred by SERS measurements as a function of incident angle $\theta$ and both incident and emitted polarizations. Results can be interpreted in the framework of Fresnel coefficients [13] and are further confirmed by an independent measurement using Polarization Modulation Infrared Reflection-Absorption Spectroscopy (PM-IRRAS) [57]. The advantage of our approach here is that it is self-normalizing and thus avoids several limitations: $(i)$ it does not require the SERS EFs (even relative EFs among modes), and is therefore not affected by modifications of the tensors upon adsorption, $(i i)$ it does not require modes with different symmetries, thus allowing the study of resonant molecules, and ( $\mathrm{ii}$ i) it is not influenced by the plasmon resonance dispersion $[47,55,56]$. The advantages, however, do not come without limitations, which will be discussed.

Given the fact that the modification of the Raman signal for a flat metallic surface illuminated from air is not due to a plasmonic effect and enhancement factors are mostly smaller than 1, one may argue that the work of this chapter should, strictly speaking, not be referred to in the context of SERS. But since the focus is on the verification of SSR for SERS a change in terminology (to Surface modified Raman Scattering) would only add confusion to a already challenging subject. Hence it shall only be emphasized at this point that the experimental situation we focus on in this chapter does not involve surface plasmon excitation and that furthermore the local field intensities are mostly quenched. 


\subsection{Theory: the angular emission profile}

In Section 1.3 of Chapter 1 an expression for the SMEF $F$ for a molecule on a metallic surface was derived. In order to evaluate this expression and obtain an explicit value for the enhancement factor, it is not only necessary to know the local fields at the molecule's position, but also its normalized polarizability tensor $\hat{\alpha}_{N}$. Since $\hat{\alpha}_{N}$ is in general fairly complicated, it is quite difficult to obtain such a value for $F$ - even for the case of a flat surface where the local fields are fairly easy to predict numerically. If we assume, however, that $\hat{\alpha}_{N}$ is uni-axial, the expression for $F$ can be simplified allowing us (with the help of the SPLaC codes, see appendix F of reference [2]) to predict the emitted power $P_{\operatorname{rad}}(\theta)$ of a monolayer of molecules adsorbed on the flat metallic surface. Comparing the theoretical prediction with the experimental result will then allow us to determine the molecules orientation. This section shows

1. the analytical simplification of $F$ for a uni-axial Raman-tensor in 2 different orientations

2. the numerical evaluation of $F$ for 4 different polarization configurations;

3. the analytical expression of $P_{\mathrm{rad}}(\theta)$ for a monolayer of molecules;

4. the numerical procedure to evaluate $P_{\mathrm{rad}}(\theta)$

The point of this is to show for a simple system the connection of the local field enhancement factor $M_{\text {loc }}$ and the single molecule enhancement factor $F$.

\subsubsection{The SERS EF for a uniaxial tensor}

For excitation at a given incident polarization $\mathbf{P}_{\mathrm{L}}\left(\mathbf{P}_{\mathrm{L}}=\mathrm{TM}\right.$ or TE), incident angle $\theta_{\mathrm{L}}$ and incident wavelength $\lambda_{\mathrm{L}}$ the local field $\mathbf{E}_{\mathrm{Loc}}^{\mathbf{P}_{\mathrm{L}}}\left(\lambda_{\mathrm{L}}, \theta_{\mathrm{L}}\right)$ at the molecules position induces a Raman dipole $\mathbf{p}=\hat{\alpha}$. $\mathbf{E}_{\mathrm{Loc}}^{\mathbf{P}_{\mathrm{L}}}\left(\lambda_{\mathrm{L}}, \theta_{\mathrm{L}}\right)$ (where $\hat{\alpha}$ is the Raman polarizability tensor). It is the emission of this dipole (modified by the presence of the metal) that we detect as a SERS signal at the Raman-shifted wavelength $\lambda_{R}$. If we analyze this emitted light along a given polarization $\mathbf{P}_{R}\left(\mathbf{P}_{R}=T M\right.$ or TE$)$ and in a given direction $\theta_{\mathrm{R}}$, the SERS enhancement factor can be expressed as in eq. 1.48:

$$
F^{\mathbf{P}_{\mathrm{L}}-\mathbf{P}_{\mathrm{R}}}=\left|\hat{\mathbf{E}}_{\mathrm{Loc}}^{\mathbf{P}_{\mathrm{R}}}\left(\lambda_{\mathrm{R}}, \theta_{\mathrm{R}}\right) \cdot \hat{\alpha}_{\mathrm{N}} \cdot \hat{\mathbf{E}}_{\mathrm{Loc}}^{\mathbf{P}_{\mathrm{L}}}\left(\lambda_{\mathrm{L}}, \theta_{\mathrm{L}}\right)\right|^{2}
$$

Here $\hat{\alpha}_{N}$ is the normalized Raman polarizability tensor and $\hat{\mathbf{E}}_{\mathrm{Loc}}^{\mathbf{P}}$ is the normalized local field:

$$
\hat{\mathbf{E}}_{\mathrm{Loc}}^{\mathbf{P}} \equiv \frac{\mathbf{E}_{\mathrm{Loc}}^{\mathbf{P}}}{\left|\mathbf{E}_{\text {Inc }}\right|}
$$

As already mentioned in the introduction to this section, for a general $\hat{\alpha}_{\mathrm{N}}$ this expression can be fairly complicated which leads us to consider two simpler cases in the following. 


\section{Uniaxial tensor perpendicular to the metallic plane}

For an uniaxial tensor $\hat{\alpha}_{\mathrm{N}}^{\mathbf{e}}$ with axis $\mathbf{e}$ perpendicular to the plane (i.e. along $\mathbf{e}_{z}$ ), trivially all tensor components are zero except $\left(\hat{\alpha}_{\mathrm{N}}^{\mathrm{e} z}\right)_{z z}=\sqrt{15 / 4}$ (see Section 1.2):

$$
\hat{\alpha}_{\mathrm{N}}^{\mathbf{e}_{z}}=\left(\begin{array}{ccc}
0 & 0 & 0 \\
0 & 0 & 0 \\
0 & 0 & \sqrt{\frac{15}{4}}
\end{array}\right)
$$

In this case, it is easy to see with eq. 3.1, that:

$$
F_{\perp}^{\mathbf{P}_{\mathrm{L}}-\mathbf{P}_{\mathrm{R}}}=\left|\left(\hat{\mathbf{E}}_{\mathrm{Loc}}^{\mathbf{P}_{\mathrm{R}}}\left(\lambda_{\mathrm{R}}, \theta_{\mathrm{R}}\right)\right)_{z} \sqrt{\frac{15}{4}}\left(\hat{\mathbf{E}}_{\mathrm{Loc}}^{\mathbf{P}_{\mathrm{L}}}\left(\lambda_{\mathrm{L}}, \theta_{\mathrm{L}}\right)\right)_{z}\right|^{2}
$$

With the definition of the perpendicular local field intensity enhancement $M_{\perp}^{\mathbf{P}} \equiv\left|\left(\hat{\mathbf{E}}_{\mathrm{Loc}}^{\mathbf{P}}\right)_{z}\right|^{2}$ it follows that:

$$
F_{\perp}^{\mathbf{P}_{\mathrm{L}}-\mathbf{P}_{\mathrm{R}}}=\frac{15}{4} M_{\perp}^{\mathbf{P}_{\mathrm{R}}}\left(\lambda_{\mathrm{R}}, \theta_{\mathrm{R}}\right) M_{\perp}^{\mathbf{P}_{\mathrm{L}}}\left(\lambda_{\mathrm{L}}, \theta_{\mathrm{L}}\right)
$$

Since $M_{\perp}^{\mathrm{TE}}=0$, it is easy to see that $F_{\perp}^{\mathrm{TE}-\mathrm{TM}}=F_{\perp}^{\mathrm{TM}-\mathrm{TE}}=F_{\perp}^{\mathrm{TE}-\mathrm{TE}}=0$ in this particular case.

\section{Uniaxial tensor parallel to the metallic surface}

For a uniaxial tensor with axis parallel to the metallic plane ( $x y$-plane) the expression for $\hat{\alpha}_{N}^{\mathrm{e}}$ becomes a bit more complicated as this axis may take any random orientation inside the $x y$-plane. The case of

$$
\hat{\alpha}_{\mathrm{N}}^{\mathbf{e}_{x}}=\left(\begin{array}{ccc}
\sqrt{\frac{15}{4}} & 0 & 0 \\
0 & 0 & 0 \\
0 & 0 & 0
\end{array}\right)
$$

is only one example (for orientation along the $x$ direction) taken from an infinite set $M$. Since the observed enhancement factor

$$
F_{\|}^{\mathbf{P}_{\mathrm{L}}-\mathbf{P}_{\mathrm{R}}}=\left\langle F^{\mathbf{P}_{\mathrm{L}}-\mathbf{P}_{\mathrm{R}}}\left(\hat{\alpha}_{\mathrm{N}}\right)\right\rangle_{\hat{\alpha}_{\mathrm{N}} \in M}
$$

is an average over all the elements in this set, it is important to get a better understanding of $M$. Most desirable would be a parameterization of its elements as it would allow us to evaluate eq. 3.6 analytically. Our goal is therefore firstly to find a suitable parameterization of $M$, and secondly to evaluate with this eq. 3.6.

Parameterization of $M$ : It is easy to see that $\mathbf{e}_{\phi} \equiv(\cos (\phi), \sin (\phi), 0)$ is a unit vector in the $x y$-plane that allows us to properly parameterize $M$ :

$$
M=\left\{\hat{\alpha}_{\mathrm{N}}^{\mathbf{e}_{\phi}}: \phi \in[0,2 \pi)\right\}
$$


"Properly" in this context means that $\mathbf{e}_{\phi}$ has a uniform distribution which reflects the random orientation of the Raman-tensor. In order to find an expression for $\hat{\alpha}_{N}^{\mathbf{e}_{\phi}}$ let us consider a coordinate transformation $T_{\phi}$ that turns the coordinate system $\Sigma$ by a positive angle $\phi$ (i.e. counter-clockwise) around the $\mathbf{e}_{z}$-axis into $\tilde{\Sigma}$ :

$$
\begin{array}{lll}
\Sigma & \stackrel{T_{\phi}}{\longrightarrow} & \tilde{\Sigma} \\
x & \longmapsto & T_{\phi}(x)=T_{\phi} x
\end{array}
$$

$T_{\phi}$ (used interchangeably for the coordinate transformation itself and the matrix describing it) is canonically given by:

$$
T_{\phi}=\left(\begin{array}{ccc}
\cos (\phi) & \sin (\phi) & 0 \\
-\sin (\phi) & \cos (\phi) & 0 \\
0 & 0 & 1
\end{array}\right)
$$

It is important to remember that the Raman tensor is a tensor of second order and that, in consequence, its matrix will transform differently compared to the coordinates of a vector:

$$
\begin{array}{rll}
\Sigma & \stackrel{T_{\phi}}{\longrightarrow} & \tilde{\Sigma} \\
\hat{\alpha}_{\mathrm{N}} & \longmapsto & T_{\phi}\left(\hat{\alpha}_{\mathrm{N}}\right)=T_{\phi} \hat{\alpha}_{\mathrm{N}} T_{\phi}^{-1}
\end{array}
$$

In order to find out how $\hat{\alpha}_{\mathrm{N}}^{\mathbf{e}_{\phi}}$ looks like in $\Sigma$ for an arbitrary angle $\phi$, we can ask the equivalent question of how $\hat{\alpha}_{\mathrm{N}}^{\mathbf{e}_{x}}$ looks like in $\tilde{\Sigma}$ under the coordinate transformation $T_{-\phi}$. This can be expressed in short as:

$$
\hat{\alpha}_{\mathrm{N}}^{\mathbf{e}_{\phi}}=T_{-\phi}\left(\hat{\alpha}_{\mathrm{N}}^{\mathbf{e}_{x}}\right) \stackrel{\text { eq.(3.9) }}{=} T_{\phi}^{-1} \hat{\alpha}_{\mathrm{N}}^{\mathbf{e}_{x}} T_{\phi}
$$

Here we used that $T_{-\phi}=T_{\phi}^{-1}$. Putting the expressions for $T_{\phi}$ (eq. 3.8) and $\hat{\alpha}_{\mathrm{N}}$ (eq. 3.5) into eq. 3.10 yields:

$$
\begin{aligned}
\hat{\alpha}_{\mathrm{N}}^{\mathbf{e}_{\phi}} & =\underbrace{\left(\begin{array}{ccc}
\cos (\phi) & -\sin (\phi) & 0 \\
\sin (\phi) & \cos (\phi) & 0 \\
0 & 0 & 1
\end{array}\right)}_{T_{\phi}^{-1}} * \underbrace{\left(\begin{array}{ccc}
\sqrt{\frac{15}{4}} & 0 & 0 \\
0 & 0 & 0 \\
0 & 0 & 0
\end{array}\right)}_{\hat{\alpha}_{\mathrm{N}}^{\mathbf{e}_{x}}} * \underbrace{\left(\begin{array}{ccc}
\cos (\phi) & \sin (\phi) & 0 \\
-\sin (\phi) & \cos (\phi) & 0 \\
0 & 0 & 1
\end{array}\right)}_{T_{\phi}} \\
& =\sqrt{\frac{15}{4}} *\left(\begin{array}{ccc}
\cos ^{2}(\phi) & \sin (\phi) \cos (\phi) & 0 \\
\sin (\phi) \cos (\phi) & \sin ^{2}(\phi) & 0 \\
0 & 0 & 0
\end{array}\right)
\end{aligned}
$$

Averaging of $F^{\mathbf{P}_{\mathrm{L}}-\mathbf{P}_{\mathrm{R}}}\left(\hat{\alpha}_{\mathrm{N}}^{\mathbf{e}_{\phi}}\right)$ : Having found an explicit expression for a uniaxial tensor randomly oriented in the $x y$-plane (eq. 3.11), we are now only missing expressions for $\hat{\mathbf{E}}_{\mathrm{Loc}}^{\mathrm{TM}}$ and $\hat{\mathbf{E}}_{\mathrm{Loc}}^{\mathrm{TE}}$ in order 

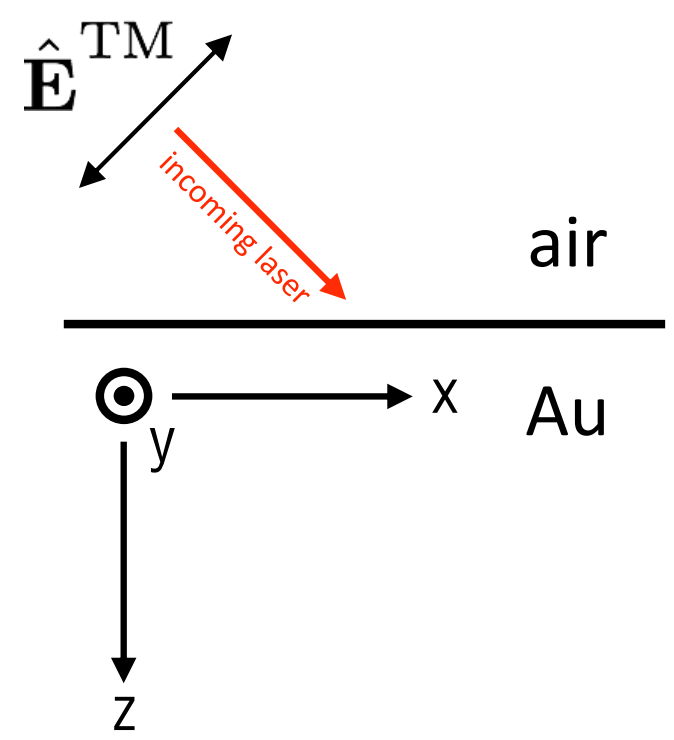

Fig. 22: Coordinate frame. to be able to evaluate eq. 3.1. Choosing our coordinate system canonically (as depicted in Fig.(22), it is easy to see that for a TM polarized wave, only the $y$-component is vanishing:

$$
\hat{\mathbf{E}}_{\mathrm{Loc}}^{\mathrm{TM}}=\left(\begin{array}{c}
E_{x} \\
0 \\
E_{z}
\end{array}\right)
$$

TE-polarized light, on the other hand, is oriented along the $y$-axis and therefore the $x$ as well as the $y$ components are vanishing:

$$
\hat{\mathbf{E}}_{\mathrm{Loc}}^{\mathrm{TE}}=\left(\begin{array}{c}
0 \\
E_{y} \\
0
\end{array}\right)
$$

It is now fairly straightforward to evaluate and average $F$ as given in eq. 3.1 for the different polarization configurations.

- $\underline{T M-T M}$ : Putting the expressions for $\hat{\mathbf{E}}_{\mathrm{Loc}}^{\mathrm{TM}}$ (eq. 3.12) and for $\hat{\alpha}_{\mathrm{N}}^{\mathbf{e}_{\phi}}$ (eq. 3.11) into eq. 3.1 yields:

$$
\begin{aligned}
F^{\mathrm{TM}-\mathrm{TM}}\left(\hat{\alpha}_{\mathrm{N}}^{\mathbf{e}_{\phi}}\right) & =\frac{15}{4}\left|\left(\begin{array}{c}
E_{x} \\
0 \\
E_{z}
\end{array}\right)^{T}\left(\begin{array}{ccc}
\cos ^{2}(\phi) & \sin (\phi) \cos (\phi) & 0 \\
\sin (\phi) \cos (\phi) & \sin ^{2}(\phi) & 0 \\
0 & 0 & 0
\end{array}\right)\left(\begin{array}{c}
\tilde{E}_{x} \\
0 \\
\tilde{E}_{z}
\end{array}\right)\right|^{2} \\
& =\frac{15}{4} \cos ^{4}(\phi)\left|\left(\hat{\mathbf{E}}_{\mathrm{Loc}, \mathrm{R}}^{\mathrm{TM}}\right)_{x}\right|^{2}\left|\left(\hat{\mathbf{E}}_{\mathrm{Loc}, \mathrm{L}}^{\mathrm{TM}}\right)_{x}\right|^{2} \\
& =\frac{15}{4} \cos ^{4}(\phi) M_{\|}^{\mathrm{TM}}\left(\lambda_{\mathrm{R}}, \theta_{\mathrm{R}}\right) M_{\|}^{\mathrm{TM}}\left(\lambda_{\mathrm{L}}, \theta_{\mathrm{L}}\right)
\end{aligned}
$$

Here the definition of $M_{\|}^{\mathbf{P}}$ was used:

$$
M_{\|}^{\mathbf{P}} \equiv\left|\left(\hat{\mathbf{E}}_{\mathrm{Loc}}^{\mathbf{P}}\right)_{x}\right|^{2}+\left|\left(\hat{\mathbf{E}}_{\mathrm{Loc}}^{\mathbf{P}}\right)_{y}\right|^{2}
$$

Eq. 3.14 can now easily be averaged:

$$
\begin{aligned}
F_{\|}^{\mathrm{TM}-\mathrm{TM}} & =\frac{15}{4} * \underbrace{\frac{1}{2 \pi} \int_{0}^{2 \pi} \cos ^{4}(\phi) \mathrm{d} \phi}_{\left\langle\cos ^{4}(\phi)\right\rangle=3 / 8} * M_{\|}^{\mathrm{TM}}\left(\lambda_{\mathrm{R}}, \theta_{\mathrm{R}}\right) M_{\|}^{\mathrm{TM}}\left(\lambda_{\mathrm{L}}, \theta_{\mathrm{L}}\right) \\
& =\frac{15}{4} * \kappa^{\mathrm{TM}-\mathrm{TM}} * M_{\|}^{\mathrm{TM}}\left(\lambda_{\mathrm{R}}, \theta_{\mathrm{R}}\right) M_{\|}^{\mathrm{TM}}\left(\lambda_{\mathrm{L}}, \theta_{\mathrm{L}}\right)
\end{aligned}
$$

In this equation $\kappa^{\mathrm{TM}-\mathrm{TM}} \equiv\left\langle\cos ^{4}(\phi)\right\rangle=3 / 8$ was introduced for the factor arising from the averaging process.

- $\underline{T E-T E: ~ J u s t ~ a s ~ i n ~ t h e ~ p r e v i o u s ~ c a s e, ~ e q . ~} 3.1$ serves as a starting point. While the expressions for $\hat{\alpha}_{\mathrm{N}}^{\boldsymbol{e}_{\phi}}$ (eq. (3.11)) remains untouched, the electric field $\hat{\mathbf{E}}_{\mathrm{Loc}}^{\mathrm{TE}}$ (eq. 3.12) differs significantly 
from the previous case:

$$
\begin{aligned}
F^{\mathrm{TE}-\mathrm{TE}}\left(\hat{\alpha}_{\mathrm{N}}^{\mathbf{e}_{\phi}}\right) & =\frac{15}{4}\left|\left(\begin{array}{c}
0 \\
E_{y} \\
0
\end{array}\right)^{T}\left(\begin{array}{ccc}
\cos ^{2}(\phi) & \sin (\phi) \cos (\phi) & 0 \\
\sin (\phi) \cos (\phi) & \sin ^{2}(\phi) & 0 \\
0 & 0 & 0
\end{array}\right)\left(\begin{array}{c}
0 \\
\tilde{E}_{y} \\
0
\end{array}\right)\right|^{2} \\
& =\frac{15}{4} \sin ^{4}(\phi)\left|\left(\hat{\mathbf{E}}_{\mathrm{Loc}, \mathrm{R}}^{\mathrm{TE}}\right)_{y}\right|^{2}\left|\left(\hat{\mathbf{E}}_{\mathrm{Loc}, \mathrm{L}}^{\mathrm{TE}}\right)_{y}\right|^{2} \\
& =\frac{15}{4} \sin ^{4}(\phi) M_{\|}^{\mathrm{TE}}\left(\lambda_{\mathrm{R}}, \theta_{\mathrm{R}}\right) M_{\|}^{\mathrm{TE}}\left(\lambda_{\mathrm{L}}, \theta_{\mathrm{L}}\right)
\end{aligned}
$$

Again the definition of $M_{\|}^{\mathbf{P}}$ (see eq. 3.15) was used for the final expression. Eq.3.17 can now be easily averaged:

$$
F_{\|}^{\mathrm{TE}-\mathrm{TE}}=\frac{15}{4} * \kappa^{\mathrm{TE}-\mathrm{TE}} * M_{\|}^{\mathrm{TE}}\left(\lambda_{\mathrm{R}}, \theta_{\mathrm{R}}\right) M_{\|}^{\mathrm{TE}}\left(\lambda_{\mathrm{L}}, \theta_{\mathrm{L}}\right)
$$

Here $\kappa^{\mathrm{TE}-\mathrm{TE}} \equiv\left\langle\sin ^{4}(\phi)\right\rangle=3 / 8$ was introduced for the factor arising from the averaging process.

- TM-TE: For the case of detection and excitation being perpendicular to one another, the evaluation of eq. 3.1 with the variables $\hat{\alpha}_{\mathrm{N}}^{\mathbf{e}_{\phi}}, \hat{\mathbf{E}}_{\mathrm{Loc}, \mathrm{R}}^{\mathrm{TE}}$ and $\hat{\mathbf{E}}_{\mathrm{Loc}, \mathrm{L}}^{\mathrm{TM}}$ (eq. 3.11 and 3.12 and 3.13 respectively) looks as follows:

$$
\begin{aligned}
F^{\mathrm{TM}-\mathrm{TE}}\left(\hat{\alpha}_{\mathrm{N}}^{\mathbf{e}_{\phi}}\right) & =\frac{15}{4}\left|\left(\begin{array}{c}
0 \\
E_{y} \\
0
\end{array}\right)^{T}\left(\begin{array}{ccc}
\cos ^{2}(\phi) & \sin (\phi) \cos (\phi) & 0 \\
\sin (\phi) \cos (\phi) & \sin ^{2}(\phi) & 0 \\
0 & 0 & 0
\end{array}\right)\left(\begin{array}{c}
\tilde{E}_{x} \\
0 \\
\tilde{E}_{z}
\end{array}\right)\right|^{2} \\
& =\frac{15}{4} \sin ^{2}(\phi) \cos ^{2}(\phi)\left|\left(\hat{\mathbf{E}}_{\mathrm{Loc}, \mathrm{R}}^{\mathrm{TE}}\right)_{y}\right|^{2} \mid\left(\hat{\mathbf{E}}_{\mathrm{Loc}, \mathrm{L})\left._{x}\right|^{\mathrm{TM}}}^{2}\right. \\
& =\frac{15}{4} \sin ^{2}(\phi) \cos ^{2}(\phi) M_{\|}^{\mathrm{TE}}\left(\lambda_{\mathrm{R}}, \theta_{\mathrm{R}}\right) M_{\|}^{\mathrm{TM}}\left(\lambda_{\mathrm{L}}, \theta_{\mathrm{L}}\right)
\end{aligned}
$$

$M_{\|}^{\mathbf{P}}$ was defined in eq. 3.15. Equation 3.19 can now be easily calculated:

$$
F_{\|}^{\mathrm{TM}-\mathrm{TE}}=\frac{15}{4} * \kappa^{\mathrm{TM}-\mathrm{TE}} * M_{\|}^{\mathrm{TE}}\left(\lambda_{\mathrm{R}}, \theta_{\mathrm{R}}\right) M_{\|}^{\mathrm{TM}}\left(\lambda_{\mathrm{L}}, \theta_{\mathrm{L}}\right)
$$

Here $\kappa^{\mathrm{TM}-\mathrm{TE}} \equiv\left\langle\sin ^{2}(\phi) \cos ^{2}(\phi)\right\rangle=1 / 8$ is now weaker by a factor of 3 compared to the configuration in which excitation- and detection-orientation are aligned (i.e. TM-TM and TE-TE).

- $\underline{T E-T M}$ : With the previous calculation it is quite obvious that:

$$
F^{\mathrm{TE}-\mathrm{TM}}\left(\hat{\alpha}_{N}^{\mathbf{e}_{\phi}}\right)=\frac{15}{4} \sin ^{2}(\phi) \cos ^{2}(\phi) M_{\|}^{\mathrm{TM}}\left(\lambda_{\mathrm{R}}, \theta_{\mathrm{R}}\right) M_{\|}^{\mathrm{TE}}\left(\lambda_{\mathrm{L}}, \theta_{\mathrm{L}}\right)
$$

After averaging this yields:

$$
F_{\|}^{\mathrm{TE}-\mathrm{TM}}=\frac{15}{4} * \kappa^{\mathrm{TE}-\mathrm{TM}} * M_{\|}^{\mathrm{TM}}\left(\lambda_{\mathrm{R}}, \theta_{\mathrm{R}}\right) M_{\|}^{\mathrm{TE}}\left(\lambda_{\mathrm{L}}, \theta_{\mathrm{L}}\right)
$$

In this equation $\kappa^{\mathrm{TE}-\mathrm{TM}} \equiv\left\langle\sin ^{2}(\phi) \cos ^{2}(\phi)\right\rangle=1 / 8=\kappa^{\mathrm{TM}-\mathrm{TE}}$. 
These detailed calculations can be summarized as follows: assuming a random orientation of the dipole axis parallel to the metallic surface, the averaged SERS enhancement factor is:

$$
F_{\|}^{\mathbf{P}_{\mathrm{L}}-\mathbf{P}_{\mathrm{R}}}=\frac{15}{4} \kappa^{\mathbf{P}_{\mathrm{L}}-\mathbf{P}_{\mathrm{R}}} M_{\|}^{\mathbf{P}_{\mathrm{L}}}\left(\lambda_{\mathrm{L}}, \theta_{\mathrm{L}}\right) M_{\|}^{\mathbf{P}_{\mathrm{R}}}\left(\lambda_{\mathrm{R}}, \theta_{\mathrm{R}}\right)
$$

The variable $\kappa^{\mathbf{P}_{\mathrm{L}}-\mathbf{P}_{\mathrm{R}}}$ results from the averaging process and is summarized in the following table:

\begin{tabular}{c|cccc} 
& TM-TM & TE-TE & TM-TE & TE-TM \\
\hline$\kappa$ & $3 / 8$ & $3 / 8$ & $1 / 8$ & $1 / 8$
\end{tabular}

\subsubsection{Numerical evaluation of the SERS EF for a uniaxial tensor}

In the last few paragraphs the expression for the SERS EF $F^{\mathbf{P}_{L}-\mathbf{P}_{R}}$ (eq. 3.1) was simplified for an uniaxial tensor oriented parallel and perpendicular to a metallic surface. This rendered us with eq. 3.4 and 3.23, expressions containing only the local-field enhancement-factors (apart from constants):

$$
\begin{aligned}
& F_{\perp}^{\mathbf{P}_{\mathrm{L}}-\mathbf{P}_{\mathrm{R}}}=\frac{15}{4} M_{\perp}^{\mathbf{P}_{\mathrm{L}}}\left(\lambda_{\mathrm{L}}, \theta_{\mathrm{L}}\right) M_{\perp}^{\mathbf{P}_{\mathrm{R}}}\left(\lambda_{\mathrm{R}}, \theta_{\mathrm{R}}\right) \\
& F_{\|}^{\mathbf{P}_{\mathrm{L}}-\mathbf{P}_{\mathrm{R}}}=\frac{15}{4} \kappa^{\mathbf{P}_{\mathrm{L}}-\mathbf{P}_{\mathrm{R}}} M_{\|}^{\mathbf{P}_{\mathrm{L}}}\left(\lambda_{\mathrm{L}}, \theta_{\mathrm{L}}\right) M_{\|}^{\mathbf{P}_{\mathrm{R}}}\left(\lambda_{\mathrm{R}}, \theta_{\mathrm{R}}\right)
\end{aligned}
$$

The variable $\kappa^{\mathbf{P}_{\mathrm{L}}-\mathbf{P}_{\mathrm{R}}}$ has been summarized in Tab. 3.24. For each orientation (i.e. $\|$ and $\perp$ ), four such SERS EFs can be calculated (and measured) depending on the excitation-detection polarizations, i.e.: $\mathbf{P}_{\mathrm{L}}$ and $\mathbf{P}_{\mathrm{R}}$ being either TM or TE.

Calculations for the local electric field were carried out using the SPlaC codes (as detailed in appendix F of reference [2]) on a multilayer structure that matches our Au film samples including all intermediate layers (for example, the titanium adhesion layer to the glass substrate). Input is a plane wave with unit electric field amplitude that impinges the Au surface from the air side $(n=1)$ at an angle of incidence $\theta$ (measured from the normal). The multilayer we used are composed of:

- a $45 \mathrm{~nm}$ thick Au film, whose dielectric function is obtained from a fit of experimental data to a Drude model with two critical points $[2,58]$;

- a $2 \mathrm{~nm}$ titanium adhesion layer with dielectric function $\epsilon=-3.918+12.596 i$ at $633 \mathrm{~nm}$ and $\epsilon=-4.031+13.48 i$ at $658 \mathrm{~nm}$ (the Raman wavelength for the $590 \mathrm{~cm}^{-1}$ mode of NB);

- a semi-infinite glass ( $n=1.52)$ medium (the substrate).

Even though the program calculates the normalized local electric field $\hat{\mathbf{E}}_{\mathrm{Loc}}^{\mathrm{TM}}(\lambda, \theta)$ and $\mathbf{E}_{\mathrm{Loc}}^{\mathrm{TE}}(\lambda, \theta)$ at all interfaces as a function of wavelength $\lambda$ and angle $\theta$ for both TM and TE polarizations, here we are only interested in the air/Au interface (on the air side). Examples of the angular dependence of these quantities are given in Fig. 23. We note that $M_{\perp}^{\mathrm{TE}}=0$ in this problem and that because of the relatively thick Au layer, the results are in fact very similar to those obtained for a simple air/Au interface. 


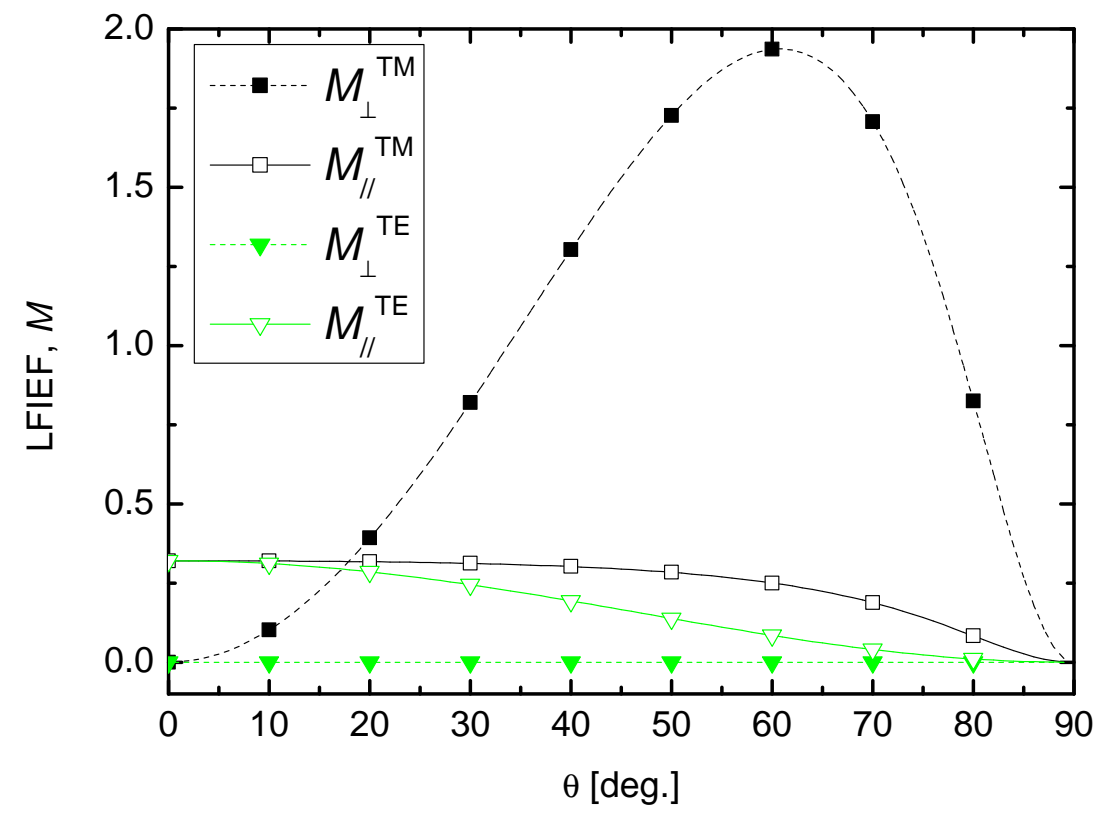

Fig. 23 :

Angular dependence of the LFEF $M_{\mathrm{loc}}(\theta)$ for the perpendicular (dashed lines, full symbols) and parallel (solid lines, hollow symbols) components of the electric field, excited with a TE- (triangles) or TM- (squares) polarized plane wave (see eq. 1.45 for definition of $M_{\text {loc }}$.

For our problem of interest here, we may assume in a first approximation that $\theta \equiv \theta_{\mathrm{L}}=\theta_{\mathrm{R}}$ (i.e. a collimated beam and BS configuration) and take $\lambda_{\mathrm{L}}=633 \mathrm{~nm}$ and $\lambda_{\mathrm{R}}=658 \mathrm{~nm}$ (corresponding to the $590 \mathrm{~cm}^{-1}$ of NB). The resulting predicted SERS EF for the four polarization configurations, and in the two extreme cases of out-of-plane and in-plane uniaxial tensors, are shown in Fig. 24. It is clear from these predictions that even a small $\alpha_{z z}$ component in the Raman tensor will strongly dominate the TM-TM signal, but should not contribute to the other three configurations.

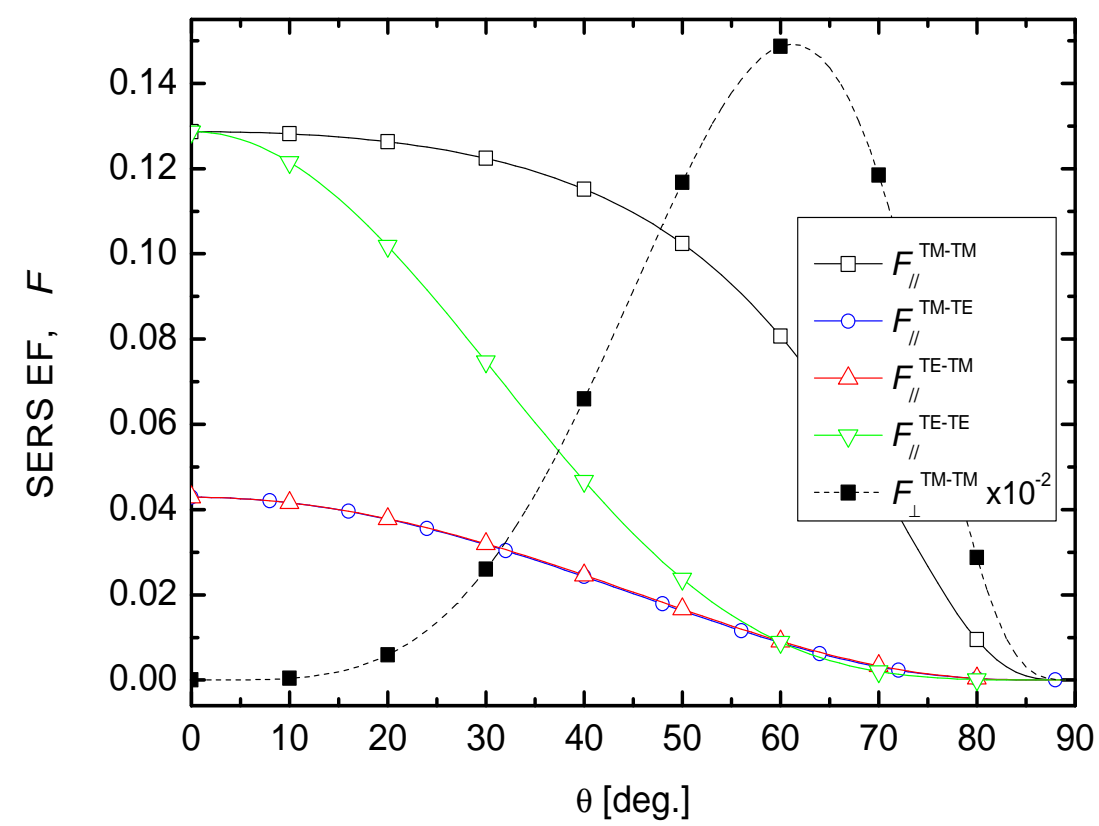

Fig. 24 :

Angular dependence of the SERS enhancement factors $F(\theta)$ for the $590 \mathrm{~cm}^{-1} \mathrm{NB} R a$ man mode for the four excitation-detection polarizations (TM-TM, TM$T E, T E-T M, T E-T E)$, and two different uniaxial Raman tensors: in-plane (II) and out-of-plane ( $\perp$ ) as specified in eq. 3.4 and eq.3.23 assuming a BS configuration with $\theta \equiv \theta_{\mathrm{L}}=\theta_{\mathrm{R}}$. 


\subsubsection{Prediction of SERS intensities for a monolayer of molecules}

The simplicity of the substrate allowed us in the previous section to model accurately the angular dependence of the LFEF $M(\theta)$. Assuming that only a single, uniaxial molecule is illuminated with a parallel beam and its signal collected in the BS-direction furthermore permitted us to set $\theta \equiv \theta_{\mathrm{L}}=$ $\theta_{\mathrm{R}}$ and calculate the SERS EFs $F(\theta)$. Knowing $F(\theta)$ and with the definition in Chapter 1 it is straight forward to write down explicitly the power $P_{\mathrm{rad}}^{\mathrm{SM}_{\mathrm{x}}}(\theta)$ emitted by a single molecule at position $\mathbf{x}$ on the metallic surface into a small solid angle of detection $\Delta \Omega_{\mathrm{R}}$ :

$$
P_{\mathrm{rad}}^{\mathrm{SM}_{\mathrm{x}}}(\theta)=p^{\mathrm{inc}}(\mathbf{x}) \cdot \frac{\mathrm{d} \sigma}{\mathrm{d} \Omega} \cdot F(\theta) \cdot \Delta \Omega_{\mathrm{R}}
$$

Even though for the assumptions made eq. 3.25 is exact (i.e. it does not rely on the $|E|^{4}$ approximation), it may not be very accurate for a realistic situation in which a homogeneous layer of molecules (rather than a single molecule) is irradiated by a cone of light (rather than a parallel beam) and the Raman signal is collected in an even larger cone (rather than just in the BS direction) as depicted in Fig. 26. Hence from now on we will distinguish the angle $\alpha$ between beam-axis and surface normal from the angle $\theta_{\mathrm{L}} / \theta_{\mathrm{R}}$ between an individual incoming / outgoing beam.

\section{Simplistic situation}

In order to predict the angular dependence of the SERS signal for a layer of molecules with uniform density $\rho$ (incoming beam is still assumed to be parallel, collection is still assumed to happen in BS-direction), we need to integrate over the illuminated surface and take into account the geometrical shape of our incident beam. Since its size on the sample changes with incident angle $\alpha$, the number of molecules in the beam changes as well which needs to be accounted for by introducing an effective molecule density $\rho_{\text {eff }}=\rho / \cos (\alpha)$ which yields:

$$
\begin{aligned}
P_{\mathrm{rad}}^{\rho}(\alpha) & =\int\left(\rho_{\mathrm{eff}} \cdot P_{\mathrm{rad}}^{\mathrm{SM}_{\mathrm{x}}}(\alpha)\right) \mathrm{d} A \\
& \stackrel{(3.25)}{=} \rho \cdot P^{\mathrm{inc}} \cdot \frac{\mathrm{d} \sigma}{\mathrm{d} \Omega} \cdot \frac{F(\alpha)}{\cos (\alpha)} \cdot \Delta \Omega_{\mathrm{R}}
\end{aligned}
$$

In this equation $P^{\text {inc }} \equiv \int p^{\text {inc }}(\mathbf{x}) \mathrm{d} A$ is the total power of the laser on the sample. The values for the resulting effective enhancement factor $F(\alpha) / \cos \alpha$ are plotted in Fig. 25.

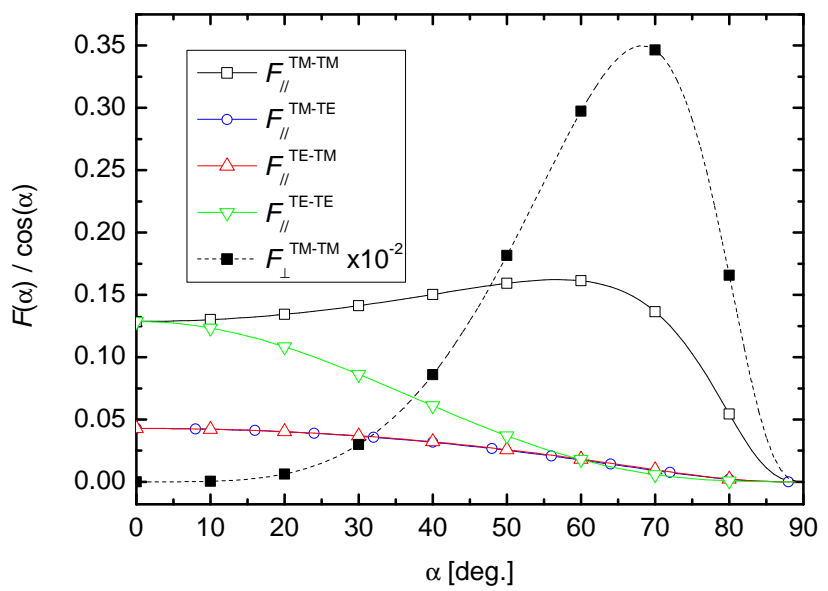

Fig. 25 :

SERS EF, F, as in Fig. 24, including the geometrical correction $1 / \cos (\alpha)$ for the excitation shape. 
The most important aspect about the theoretical results shown in Fig. 25 is that the SERS signal associated with $\perp$ components of the uniaxial Raman tensor $\left(\alpha_{z z}\right)$ clearly dominates (by a factor of $\sim 100$ ) the signal in TM-TM configuration, but is absent from the other three (TM-TE, TE-TM, and TE-TE) which are dominated by in-plane components. Its angular dependence is also very different to the other ones, going from zero at $\alpha=0$ to a maximum around $\alpha \sim 60^{\circ}$. A measurement of the TM-TM signal and of one of the other three configurations should therefore provide a clear indication of the importance of perpendicular components relative to in-plane ones.

\section{Realistic situation}

So far the angular spread of the incoming laser light and the collected Raman signal were assumed to be negligible. Fig. 26 shows a more realistic experimental situation where angular spread has to be taken into consideration and $\alpha, \theta_{\mathrm{L}}$ and $\theta_{\mathrm{R}}$ are not necessarily equal.

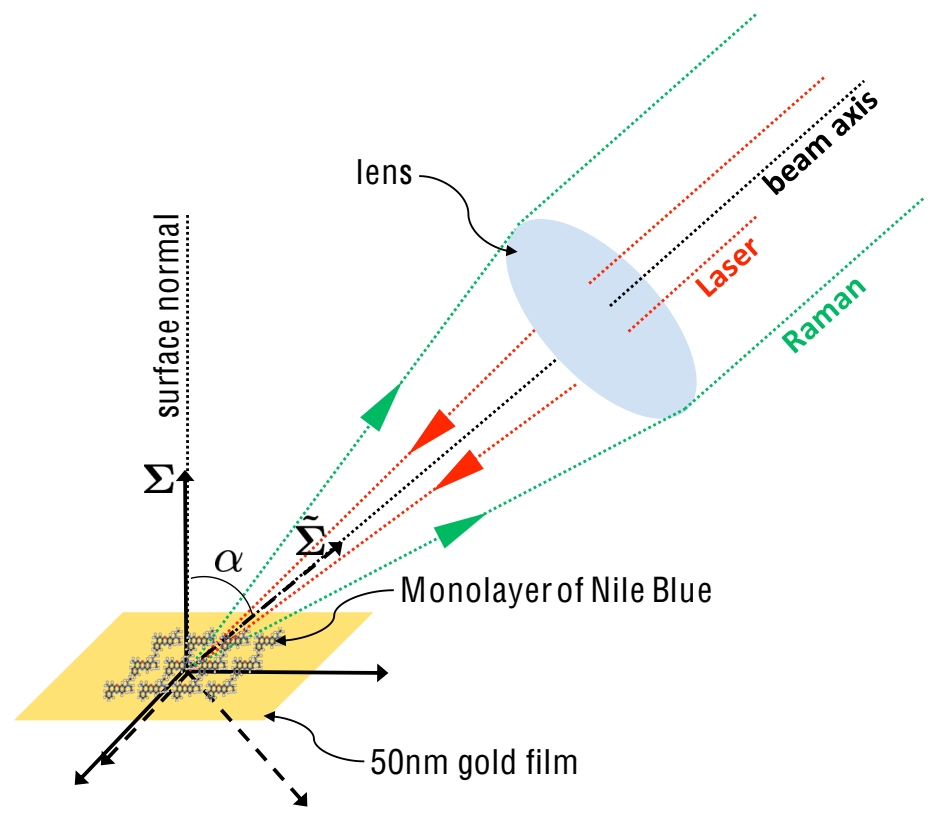

Fig. 26 :

Schematic illustration of a realistic experimental situation in which a monolayer of Nile Blue deposited on a flat Au surface is illuminated by a cone of laser-light with opening angle $\gamma_{\text {Las. }}$. The Raman signal, in turn, is collected within a cone of opening angle $\gamma_{\mathrm{Obj}}$.

This is particularly important for the situation of normal incidence where for the case of a uniaxial tensor oriented $\perp$ to the metal surface we would otherwise predict no signal. Using eq. 3.4 and 3.23 we can write eq. 3.26 in terms of the local field enhancement factor $M$ :

$$
P_{\text {rad }}^{\rho}(\alpha)=\rho \cdot \frac{\mathrm{d} \sigma}{\mathrm{d} \Omega} \cdot K_{\#} \cdot \underbrace{\frac{1}{\cos (\alpha)} M_{\#}^{\mathbf{P}_{\mathrm{L}}}\left(\theta_{\mathrm{L}}\right) P^{\mathrm{inc}}}_{\text {excitation }} \cdot \underbrace{M_{\#}^{\mathbf{P}_{\mathrm{R}}}\left(\theta_{\mathrm{R}}\right) \Delta \Omega_{\mathrm{R}}}_{\text {detection }}
$$

Here the symbol \# can either be \# $\equiv \perp$ or \# $\equiv \|$, depending on the molecule orientation to be considered. In order to simplify things the constant $K_{\#} \equiv \frac{15}{4} \cdot \kappa_{\#}^{\mathbf{P}_{\mathrm{L}}-\mathbf{P}_{\mathrm{R}}}$ has been introduced (the values for $\kappa_{\|}^{\mathbf{P}_{\mathrm{L}}-\mathbf{P}_{\mathrm{R}}}$ are summarized in Tab. 3.24 and $\kappa_{\perp}=1$ ).

This allows us to look at excitation and emission separately: the laser power $P^{\text {inc }}$ in eq. 3.27 has to be replaced by $\Delta P^{\text {inc }}=\frac{\mathrm{d} P^{\text {inc }}}{\mathrm{d} \Omega}\left(\Omega_{\mathrm{L}}\right) \cdot \Delta \Omega_{\mathrm{L}}$ which would then describe the emitted signal due to excitation from a small solid angle $\Delta \Omega_{\mathrm{L}}$ and collection into a similarly small solid angle $\Delta \Omega_{\mathrm{R}}$. To 
obtain the total signal $P_{\mathrm{Rad}}^{\rho}(\alpha)$ as a function of the sample's angle $\alpha$ with respect to the beam axis (see Fig. 26) one has to sum over all excitation and detection angles yielding:

$$
P_{\mathrm{Rad}}^{\rho}(\alpha)=\rho \cdot \frac{\mathrm{d} \sigma}{\mathrm{d} \Omega} \cdot K_{\#} \cdot \underbrace{\int_{E\left(\alpha, N A_{\text {Las }}\right)}\left(\frac{1}{\cos (\alpha)} M_{\#}^{\mathbf{P}_{\mathrm{L}}}\left(\Omega^{\prime}\right) \cdot \frac{\mathrm{d} P^{\mathrm{inc}}}{\mathrm{d} \Omega}\left(\Omega^{\prime}\right)\right) \mathrm{d} \Omega^{\prime}}_{\text {excitation }} \cdot \underbrace{\int_{D\left(\alpha, N A_{\text {Obj }}\right)} M_{\#}^{\mathbf{P}_{\mathrm{R}}}\left(\Omega^{\prime}\right) \mathrm{d} \Omega^{\prime}}_{\text {emission }}
$$

In this equation " $E$ " and " $D$ " are sets of points on the unit sphere that are in the "field of Excitation" and in the "field of Detection" respectively. To define these sets more clearly, let us consider a point $\Omega \equiv(\theta, \phi, 1)$ on the unit-sphere with spherical coordinates $\tilde{\Sigma}_{\alpha}(\Omega)=\tilde{\Omega} \equiv(\tilde{\theta}, \tilde{\phi}, 1)$ in the objective's reference frame $\tilde{\Sigma}$ (see Fig. 26). Here the function $\tilde{\Sigma}_{\alpha}(\cdot)$ shall assign to an arbitrary, physical point its spherical coordinates in a frame tilted by the angle $\alpha$. Quite clearly, if $\tilde{\theta}$ (its inclination angle or latitude) is smaller than the opening angle of the objective $\gamma_{\mathrm{obj}}$, then $\Omega$ is in the field of detection. Since $\gamma_{\mathrm{Obj}}$ is given by the numerical aperture (NA) of the objective $N A_{\mathrm{Obj}}$ with $N A_{\mathrm{Obj}} \equiv n_{\text {air }} \sin \left(\gamma_{\mathrm{Obj}}\right)$ this can be written in short:

$$
D\left(\alpha, N A_{\mathrm{Obj}}\right) \equiv\left\{\Omega: \quad \tilde{\theta} \leq \gamma_{\mathrm{Obj}}\right\}
$$

Similarly the field of excitation can be defined. Here, however, it is important to remember that the laser may not completely fill the back-side of the objective and that therefore the effective NA of the laser $N A_{\text {Las }}$ may be smaller than the available $N A_{\text {Obj }}$ of the objective. How close those two values are together depends crucially on the beam-diameter. Let us define the opening angle of the laser $\gamma_{\text {Las }}$ as $\gamma_{\text {Las }} \equiv \arcsin \left(N A_{\text {Las }}\right)$. It follows that:

$$
E\left(\alpha, N A_{\text {Las }}\right) \equiv\left\{\Omega: \quad \tilde{\theta} \leq \gamma_{\text {Las }}\right\}
$$

With the proper definition of the integration sets, eq.3.28 can now be evaluated numerically to obtain the SERS EF $F(\alpha):=P_{\mathrm{Rad}}^{\rho}(\alpha) /\left(\rho \cdot \mathrm{d}_{\Omega} \sigma \cdot P^{\mathrm{inc}} \cdot \Delta \Omega_{\mathrm{R}}\right)$ where $\Delta \Omega_{\mathrm{R}}=2 \pi\left(1-\cos \left(\gamma_{\text {Obj }}\right)\right)$ is the size of the field of detection $D$. This additional correction, although small, was applied to obtain the numbers in Fig. 27 and the fits of Fig. 29 and 30 for the experimental situation in which a $\times 20$ objective $\left(N A_{\mathrm{x} 20}=0.35\right)$ is used.

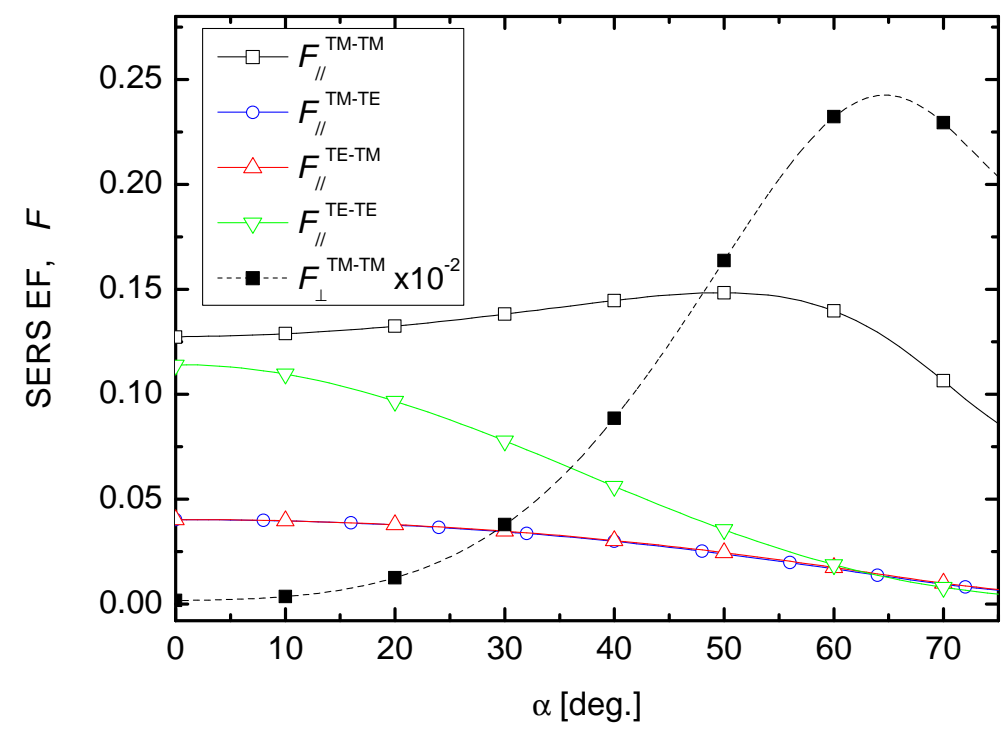

Fig. 27 :

Predictions for the angleand polarization dependence of the SERS signal assuming a uniaxial Raman tensor with main axis either $\perp$ or $\|$ (with orientation averaging) to the Au surface. 


\subsubsection{Numerical strategy to evaluate the angular emission profile}

Having derived eq. 3.28 which describes the signal expected from a layer of uniaxial molecules deposited on a Au slide, we are now in a position to sketch how to calculate this expression numerically. Note that foremost we are interested in its dependency on $\alpha$, the angle between the surface normal and the incoming beam axis.

On the unit sphere in $\tilde{\Sigma}$ (see Fig. 26 for definition) a segment defined by the numerical aperture of the lens is considered. On this segment a physical grid of $N$ points can be defined, each of which has a set of "properties" such as:

- the $\tilde{x}, \tilde{y}, \tilde{z}, \tilde{\theta}, \tilde{\phi}, \tilde{r}$ coordinates in $\tilde{\Sigma}$,

- the $x, y, z, \theta, \phi, r$ coordinates in $\Sigma$,

- the solid angles $\Delta \Omega$ centered around each grid point,

- the power density $p_{\Delta \Omega}$ at each grid point,

- the power $P_{\Delta \Omega}$ going through each window $\Delta \Omega$,

- the LFEFs $M\left(\tilde{\theta}, \lambda_{\mathrm{R}}\right)$ and $M\left(\tilde{\theta}, \lambda_{\mathrm{L}}\right)$.

The easiest way to work with these properties is to write them in matrices $A^{x}, A^{y}, \ldots, A^{\tilde{\phi}}, A^{\tilde{r}}, A^{M}$. Note that each matrix-element $A_{i, j}$ belongs to one and only one physical point on the grid (i.e. the relation between matrix elements and grid point is bijective).

Usually one starts by defining the grid in spherical coordinates in $\tilde{\Sigma}$ by defining $A^{\tilde{\theta}}$ and $A^{\tilde{\phi}}$. As can be easily seen, with a simple coordinate transformation each pair $(\tilde{\theta}, \tilde{\phi})$ can be mapped onto a pair $(\theta, \phi)$ using the equations:

$$
\begin{aligned}
\tilde{\phi} & =\arctan \left(\cos \alpha \tan \phi+\sin \theta_{\text {inc }} \frac{\cot \theta}{\cos \phi}\right) \\
\tilde{\theta} & =\arctan \left[\left(-\sin \alpha \tan \phi+\cos \theta_{\text {inc }} \frac{\cot \theta}{\cos \phi}\right) \cos \tilde{\phi}\right]^{-1}
\end{aligned}
$$

With $\Omega$ and $\tilde{\Omega}$ it is now fairly easy to calculate numerically the effective LFEF $M_{\#}^{\text {eff- } \mathbf{P}_{\mathrm{L}}}$ and $M_{\#}^{\text {eff- } \mathbf{P}_{\mathrm{R}}}$ which are defined for excitation and detection (see eq. 3.28) as follows:

$$
\begin{aligned}
& \int_{E\left(\alpha, N A_{\text {Las }}\right)} \frac{1}{\cos (\alpha)} M_{\#}^{\mathbf{P}_{\mathrm{L}}}\left(\Omega^{\prime}\right) \frac{\mathrm{d} P^{\text {inc }}}{\mathrm{d} \Omega}\left(\Omega^{\prime}\right) \mathrm{d} \Omega^{\prime} \quad=: \quad M_{\#}^{\text {eff- } \mathbf{P}_{\mathrm{L}}}(\alpha) \cdot P^{\text {inc }} \\
& \int_{D\left(\alpha, N A_{\text {Obj }}\right)} M_{\#}^{\mathbf{P}_{\mathrm{R}}}\left(\Omega^{\prime}\right) \mathrm{d} \Omega^{\prime} \quad=: \quad M_{\#}^{\text {eff- } \mathbf{P}_{\mathrm{R}}}(\alpha) \cdot \Delta \Omega_{\mathrm{R}}
\end{aligned}
$$

As before we replaced in these expression the dependency of $M_{\#}^{\text {eff- }} \mathbf{P}_{\mathrm{L}}$ and $M_{\#}^{\text {eff- } \mathbf{P}_{\mathrm{R}}}$ on molecule orientation ( $\perp$ or $\|$ ) with the \#-symbol, and omitted excitation wavelength $\lambda_{\mathrm{L}}$ and Raman wavelength 
$\lambda_{\mathrm{R}}$. This is to underline the angular dependency of $M_{\#}^{\text {eff }}$ on $\alpha$ which allows us to predict the signal $P_{\mathrm{Rad}}^{\rho}(\alpha)$ as the Au sample is being turned:

$$
P_{\mathrm{Rad}}^{\rho}(\alpha) \sim K_{\#} \cdot M_{\#}^{\mathrm{eff}-\mathbf{P}_{\mathrm{L}}}(\alpha) \cdot M_{\#}^{\mathrm{eff}-\mathbf{P}_{\mathrm{R}}}(\alpha)
$$

To account for situations in which some molecules are oriented $\perp$ and others $\|$, we use the following expression with $a \in[0,1]$ as a free fitting parameter:

$$
F(\alpha)=a *\left(K_{\|} \cdot M_{\|}^{\text {eff- } \mathbf{P}_{\mathrm{L}}} \cdot M_{\|}^{\text {eff- } \mathbf{P}_{\mathrm{R}}}\right)+(1-a)\left(K_{\perp} \cdot M_{\perp}^{\mathrm{eff}-\mathbf{P}_{\mathrm{L}}} \cdot M_{\perp}^{\text {eff- } \mathbf{P}_{\mathrm{R}}}\right)
$$

Finally two technical notes: while it is possible to use the same grid for excitation and detection we found that it is better to use separate grids as the opening angle of the incoming laser (excitation) is significantly smaller than the opening angle of the lens (detection). We also saw that it is important to include the $2 \mathrm{~nm}$ titanium layer (which is often used to have a better adhesion of Au on glass) for calculation of the LFEF $F$. 


\subsection{Experiment}

\subsubsection{Setup and sample preparation}

A Au film $(45 \mathrm{~nm}$ thickness evaporated on glass with an intermediate $2 \mathrm{~nm}$ titanium adhesion layer) was immersed in a $100 \mu \mathrm{M}$ NB solution in water for $\sim 30 \mathrm{~min}$ and then thoroughly washed with ultra-pure water and dried. Films prepared with this method achieve a monolayer deposition on the surface (corroborated by independent electrochemical measurements) and present a good overall uniformity of the SERS signal at different positions. The Raman measurements for this experiment were carried out on a Jobin-Yvon LabRam spectrometer equipped with an Olympus BX41 microscope and a notch filter for $633 \mathrm{~nm}$ laser excitation. Polarization elements $(\lambda / 2$ waveplate and polarizers) were used to set the incoming and outgoing polarization directions.

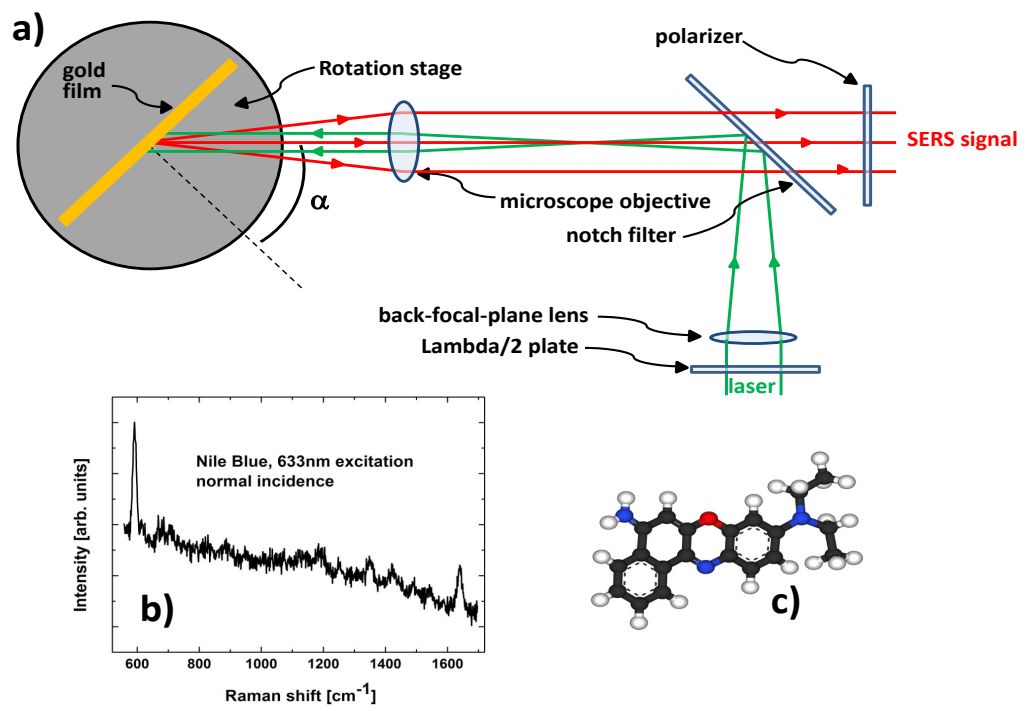

Fig. 28: (a) Experimental setup. (b) Representative SERS spectrum of $N B$, obtained at normal incidence, in TE-TE configuration, integration time $10 \mathrm{~s}$. (c) $\mathrm{NB}\left(\mathrm{C}_{20} \mathrm{H}_{20} \mathrm{~N}_{3} \mathrm{O}\right.$, for colourcode see Fig. 11)
Samples were mounted and measured on a motorized rotation stage (Thorlabs) in a BS geometry allowing precise angular scans to be performed (schematic illustration in Fig. 28(a)). Several points in the sample were measured at each angle, to ensure the results are not influenced by outliers. The polarization response of the system (gratings and subsidiary optics) is also taken into account by measuring different configurations on a reference silicon sample.

To maximize the collected signal, it is desirable to use a high NA objective. However, a high NA objective presents several disadvantages: $(i)$ it results in a spread of incident angles. ( $i i)$ The small spot size increases photo-bleaching, and prevents long integration times. (ii i) It is challenging to focus on the surface at large angles. As a compromise, we used a $\times 20$ objective $\left(N A_{\mathrm{x} 20}=0.35\right)$. The first two issues were eliminated by using an additional lens (focal length $500 \mathrm{~mm}$ ) to focus the laser beam onto the back focal plane of the objective (Fig. 28a)), resulting in a larger spot $(\approx 35 \mu \mathrm{m}$ diameter ${ }^{1}$, instead of $\approx 5 \mu \mathrm{m}$ ) with negligible beam divergence. We measured the SERS intensity of the $590 \mathrm{~cm}^{-1}$ mode of NB as a function of $\alpha$ for the four possible combinations of incident and detection polarizations (each being either TM or TE).

\footnotetext{
${ }^{1}$ corresponding to the full-width at $1 / e^{2}$ of the normalized intensity $|E|^{2} /\left|E_{0}\right|^{2}$
} 


\subsubsection{EF estimation at normal incidence}

It may seem surprising at first that a SERS spectrum like the example shown in Fig. 28(b) can be obtained from a monolayer of molecules adsorbed on a flat Au film. However, a simple semiquantitative estimate gives a SERS EF of $\approx 0.1$ for this measurement, a number in agreement with the LFEF calculated in the previous section. To this end, we first assume that the non-SERS differential Raman cross-section $(d \sigma / d \Omega)$ of the Raman peaks of NB at $633 \mathrm{~nm}$ is of the order of $\sim 10^{-24} \mathrm{~cm}^{2} / \mathrm{sr}$; a typical value for dyes at resonance [21]. Secondly, the number of NB molecules in the spot can be estimated from the expected surface concentration [59] $\left(5.5 \times 10^{-11} \mathrm{~mol} / \mathrm{cm}^{2}\right)$. Thirdly, we calibrated the absolute response of our system using a $\times 50\left(N A_{x 50}=0.5\right)$ objective by characterizing its scattering volume as in reference [24], and measuring the Raman signal of nitrogen (of known Raman differential cross-section [2]) in air. Putting these numbers together, we estimate a SERS enhancement factor of the order of only $\approx 0.1$ for the spectrum of Fig 28a). Although a rough estimate, this figure is comparable to the value $(\approx 0.12)$ predicted from theory. The remarkable point here is that we can observe a monolayer of molecules on a surface where the Raman signal is actually being quenched, rather than enhanced. In fact, since this SERS EF is in agreement with EM predictions, this also provides an independent experimental proof that roughness does not contribute here to the SERS signal (it would otherwise result in a much larger SERS EF).

\subsubsection{Experimental results}

The experimental data for the angle- and polarization- dependent SERS signal are shown in Figs. 29 and 30. While the TE-TE, TM-TE, and TE-TM signals are only sensitive to $\|$ tensor components and follow the corresponding predictions rather nicely, the TM-TM dependence exhibits a maximum at large $\alpha$ 's, but not as pronounced as predicted for a $\perp$ uniaxial tensor. To be more quantitative, the data for the TM-TM configuration was fitted with a linear superposition of the $\perp$ and $\|$ predictions (as described before in Section 3.3.4, eq. 3.36).

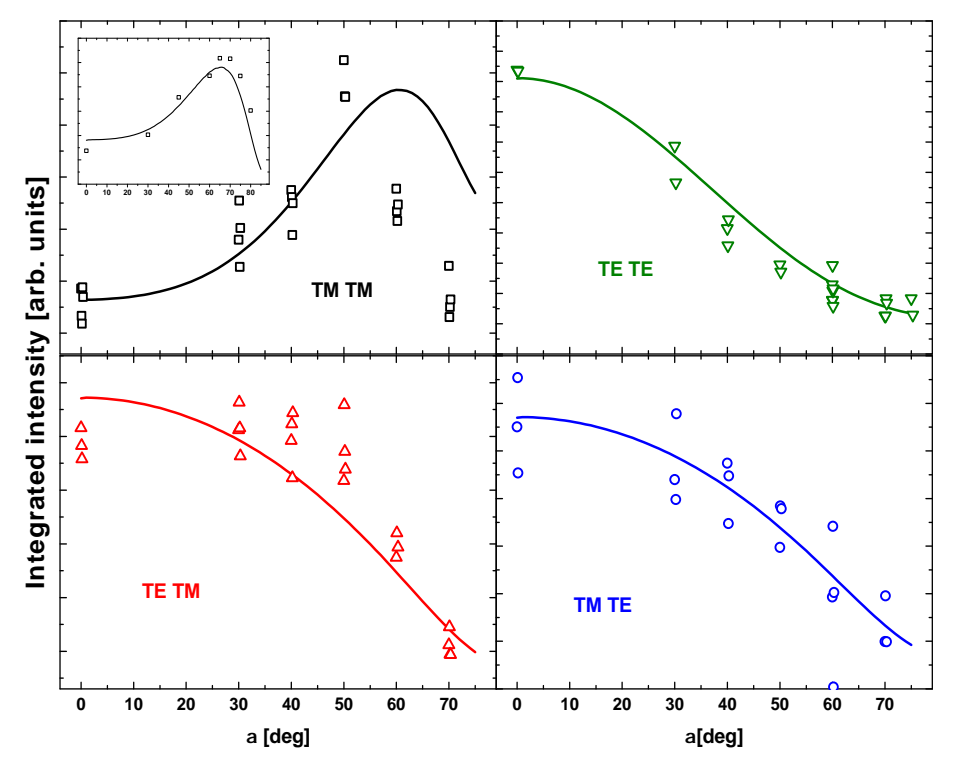

Fig. 29:

Angle-dependence of the integrated SERS intensity for the $590 \mathrm{~cm}^{-1}$ peak in the 4 polarization configurations using $a \times 20$ objective $\left(N A_{\mathrm{x} 20}=0.35\right)$. Several positions on the sample were measured for each $\alpha$. Solid lines are the theoretical predictions, assuming for TM-TM a contribution of $0.3 \%$ of $\perp$ components. The inset in shows the data and predictions for TM-TM using $a \times 4$ objective $\left(N A_{\mathrm{x} 4}=0.1\right)$. 
The superposition shown in Fig. 29 for TM-TM as a solid line indicates a fraction of only $0.3 \%$ for the $\perp$ component. Since the data taken with the $\times 4$-objective does not display such discrepancy, the deviation at large $\alpha$ 's for the $\times 20$-objective may be attributed to its depth of focus limiting the collected signal.

Similar results were obtained for the $1645 \mathrm{~cm}^{-1}$ peak of NB shown in Fig. 30, although the data are subject to more uncertainties because the signal to noise ratio in this region of the spectrum is worse. This confirms that both modes have the same Raman tensor symmetry, as expected here.

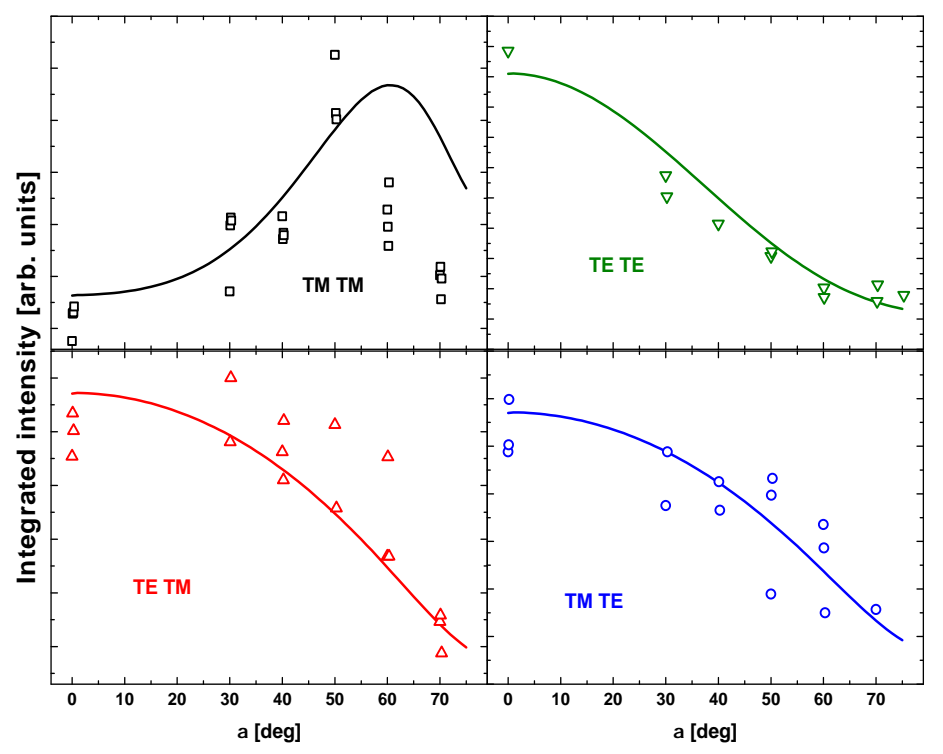

Fig. 30 :

Angle-dependence of the integrated SERS intensity for the $1645 \mathrm{~cm}^{-1}$ peak in the 4 polarization configurations using $a \times 20$ objective $\left(N A_{\mathrm{x} 20}=0.35\right)$. Several positions on the sample were measured for each $\alpha$. Solid lines are the theoretical predictions, assuming for TM-TM a contribution of $0.3 \%$ of $\perp$ components.

For NB at $633 \mathrm{~nm}$ excitation, Raman tensors are expected to follow the symmetry of the electronic state responsible for the absorption. Hence, they will have their components in the plane of the four conjugated rings of the molecule, and will (most likely) be uniaxial in this plane. We conclude that the NB molecules adsorb flat on Au; a fact expected for aromatic molecules. The small observed contribution of out-of-plane components $(0.3 \%)$ is most likely due to minute sample imperfections resulting in the occasional molecule standing upright (or with a tilt) on the surface.

\subsubsection{IR absorption and DFT study of the orientation of NB on Au}

The orientation of the adsorbed molecules was independently confirmed from a comparison of IR absorption and PM-IRRAS [57] to the predictions of DFT. Most of the IR modes that appear in the PM-IRRAS spectrum can be attributed (using DFT) to out-of-plane IR dipoles, in particular the $\mathrm{CH}$ bending modes around $800 \mathrm{~cm}^{-1}$. This confirms independently the flat adsorption geometry, and therefore the SSRs/SERS analysis. Since the PM-IRRAS measurements were carried out by our collaborators in France, the interested reader is referred to our paper [49]. 


\subsection{Discussion and conclusion}

The beauty of the experiment presented in this chapter lies in its simplicity offering a text book-like example to understand SERS in general and SSR in particular. Because of its simplicity the results obtained from it offer the most direct demonstration of the predictions first outlined by Moskovits [13] and underline the validity of the electromagnetic model and its polarization dependency used to explain SERS ${ }^{2}$.

So far most experimental studies of SSR have focused on colloidal solutions which are inherently complex and messy forcing one to introduce considerable assumptions and / or simplifications for comparison to theory. Flat surfaces allow "absolute" control of experimental conditions but are challenging due to their low enhancement factors (smaller than 1!). This quenching makes it difficult to see any signal at all and is likely to be the very reason that a study like ours has not been conducted before. It is in this context that we hope our data made a new contribution to the field of SERS.

Ideally one would use for such an experiment not only a flat surface but also no collection lens to ensure - as much as possible - parallel excitation and collimated emission. Such a setup would make comparison with theory rather easy. However, because of the low signal, we were forced to use an objective introducing convergence of the incoming beam and a collection from multiple angles. While it was possible to almost eliminate the angular spread of the incoming beam by introducing a back-focal-plane lens, the collection from multiple angles could only be accounted for numerically. Here it is important to point out, that for all but one polarization configurations not a single fitting parameter was used for the theoretical plots shown in the previous paragraphs. The excellent agreement of theory and experiment for these configurations (TE-TE, TE-TM, TMTE) only highlights how well the system is understood from an electromagnetic point of view.

Only for the data taken in the TM-TM configuration a fitting parameter had to be introduced. It represents the fraction of molecules oriented perpendicular to the surface and provides thereby a means to determine molecular orientation - a longstanding goal in SERS [50, 51]. Since we were able to confirm our finding with an independent method we hope to have created a new avenue in this regard, that would only require measuring in the TM-TM configuration (all other configurations have no $\perp$ contribution to the signal) for a few angles.

\footnotetext{
${ }^{2}$ A direct comparison with Moskovits results is challenging. On the one hand Moskovits uses the Fresnel coefficients for his calculations of signal enhancements while the equations presented here are already expressed in terms of the LFEFs (which, of course, are defined by the Fresnel coefficients). On the other hand Moskovits focuses in his discussion on cases not relevant for this chapter (i.e. a uniaxial molecule). Still, his paper outlines the theoretical framework that has been used for the calculations presented here and the best bridge between his paper and the work in this chapter is equation 5 on page 4410 of his publication [13] and eq. 3.1 of this chapter
} 


\section{Chapter 4}

\section{SERS in the Kretschmann configuration}

The work discussed in this chapter has been published in "Analytical Chemistry", 2011 [60] and in "Journal of Physical Chemistry A", 2012 [61].

\subsection{Basic idea}

A thin layer of $\mathrm{Au}$ or $\mathrm{Ag}(50 \mathrm{~nm})$ with glass on one side and a dielectric medium like air or water on the other, will be the centerpiece of theoretical and experimental considerations in this chapter. When a molecule attaches to such a thin metallic surface, the dielectric function of the ambient medium (i.e. air or water) experiences a small pertubation. This, in turn, causes a minute change in the resonance condition of the Surface Plasmon Polariton (SPP), which can be measured by observing the reflectivity. This effect is exploited in SPRS and allows one to observe adsorption kinetics.

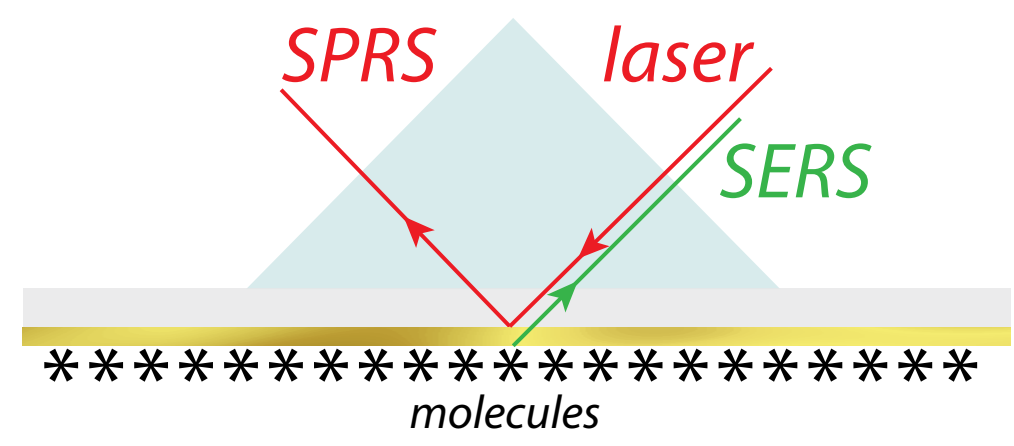

Fig. 31 : The Kretschmann configuration allowing us to combine SPRS and SERS

The Raman signal of such an adsorbed molecule can profit not only from an enhancement in excitation through the SPP, but also, by reciprocity, from an enhancement in emission. The latter has a very peculiar profile which quenches the signal on the metal side but enhances and funnels it into a narrow cone (called the Kretschmann cone) on the glass side. This opens the possibility of 
doing SERS in the Kretschmann configuration (KC) on a flat substrate with uniform, reproducible enhancement.

While SPRS is a well established technique used for bio-sensing and stands out through its excellent sensitivity [62-68], its major drawback is its inability to identify the molecules under observation. SERS, on the other hand, can do just that: the Raman spectrum allows one to identify unambiguously the molecule at hand $[20,69,70]$. Therefore it is only natural to try and combine these two, complementary techniques. That this is indeed possible will be shown in Section 4.5 with a prism setup suggested in 1968 by Kretschmann [71].

Even though this prism setup intrigues with its simplicity (it is the standard configuration for commercial instruments using SPRS) and allows one to combine SERS and SPRS with only minor modifications to a standard Raman microscope setup, it has two major drawbacks:

1. with the prism setup only a fraction of the molecule's SERS-signal can be collected;

2. with the prism setup a spatial mapping of the sample (as it is common in Raman microscopy) is almost impossible.

In Section 4.6 a new experimental setup is proposed that overcomes both those drawbacks. By directing a TM-polarized laser beam off-axis through a high NA oil-immersion-objective, it is possible to accommodate the resonance condition in excitation and collect all of the emitted signal

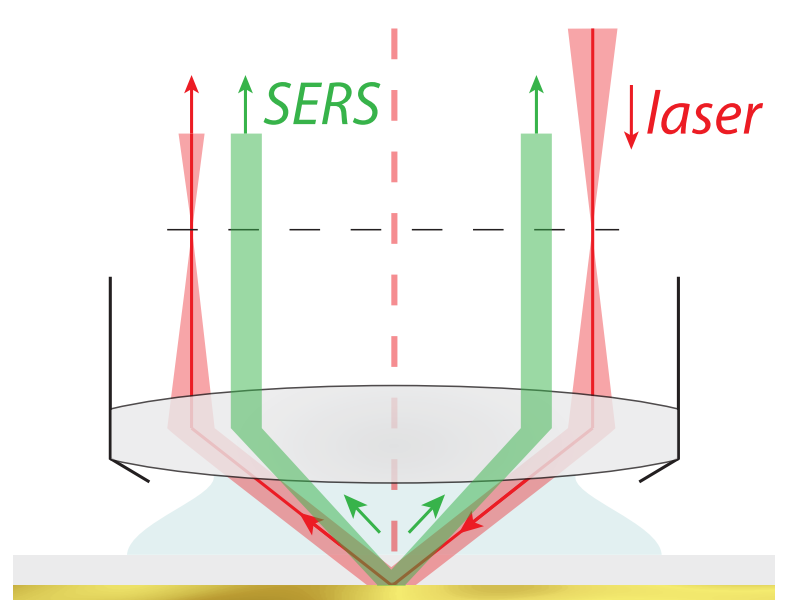

Fig. 32 : Illustration of a new Kretschmann setup using a high NA objective allowing us to collect the complete Kretschmann cone. (i.e. the whole Kretschmann cone). This not only paves the way for a novel hybrid spectroscopic technique that combines SPRS with SERS microscopy, but also constitutes a shift in paradigm: instead of chasing after ever higher enhancement factors on increasingly more complex substrates, our proposed setup uses the arguably simplest plasmonic system - a flat metal surface. This not only provides perfectly uniform enhancements but also enhancements that are well understood theoretically. All this is preceded by a proper introduction of the historical background (Section 4.2), the theory of plasmons on planar surfaces (Section 4.3) and a systematic study of possible experimental setups (Section 4.4). 


\subsection{Context}

\subsubsection{Motivation - a shift in paradigm for SERS substrates}

Since its discovery in 1974 by McQuillan and coworkers [3] SERS has come a long way, both theoretically (by identifying the effect to be of electromagnetic nature [6]) as well as experimentally (by showing that single-molecule detection is indeed possible [8-12]). Yet the fact remains, that since the early days $[7,72,73]$, progress in doing analytical work with SERS has always been thwarted by the irreproducibility of the substrates [74]. Efforts have been made in colloidal chemistry [75-77] and nano-lithography $[20,56]$ to counter this trend by tailor made probes, nano engineered substrates or both $[20,72,78-83]$. There are even a few studies that show moderate enhancements that are indeed reproducible $[20,69,70]$. But in the end a lot of the effort remained focused on achieving ever higher enhancement factors, which allowed single molecule detection in a wide variety of cases $[10,11,84-90]$. In this chase for maximum signal an overwhelming number of different substrates has sprouted [20, 72, 78, 91-95]: different designs of nanospheres, nonoarrays, nanoholes, gratings and rough surfaces usually explore how changing parameters such as geometry, dielectric material or a combination thereof can improve performance. But naturally this increase in variety leads to research efforts on these different designs becoming more isolated. Moreover the fact remains that the higher the enhancement factor achieved, the more irreproducible the substrate becomes, an empirical observation that has been dubbed by Nathan the SERS uncertainty principle [1].

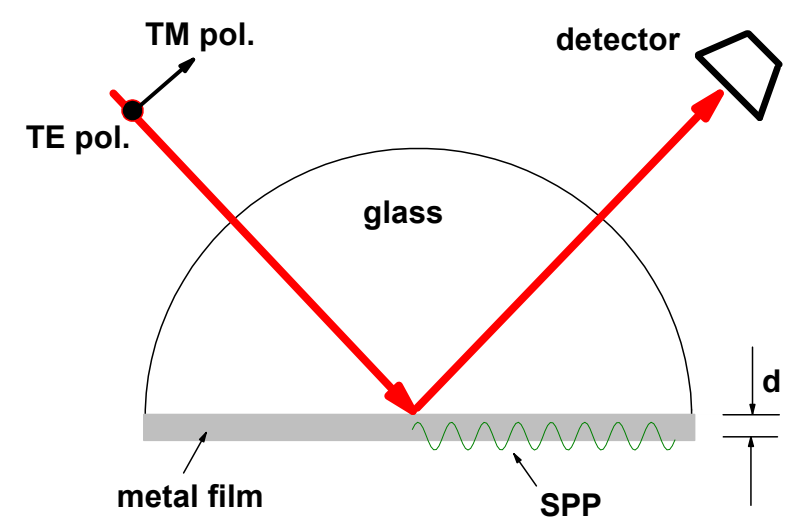

Fig. 33 : Schematics of the Kretschmann configuration [96]; when TM polarized light impinges a thin metallic film from the glass side at the resonance angle, its evanescence field can couple to a SPP on the airglass side.
Perhaps this has left a gap that we propose to address by having a closer look at flat metallic surfaces (like Au or Ag) which are arguably the simplest plasmonic system available. While excitation and emission are quenched when looking at molecules on such a surface from the front side (i.e. the air side), in the KC [71] (as depicted schematically in Fig. 33 and further explained in the text later) it is possible to excite surface plasmons and profit from considerable signal enhancements on the order of $10^{4}$. While this is nowhere near the reported maximum enhancement factors of $10^{8}-10^{10}$ available in a colloidal solution, it is enough to outperform other techniques (with fluorescence spectroscopy as the "standard" reference [97] ) and even detect single, resonant molecules (with intrinsic cross-sections of the order of $\left.\sim 10^{-24} \mathrm{~cm}^{2} / \mathrm{sr}[21,30]\right)$.

The advantages of working with a flat surface are quite apparent:

- Easy access to high quality substrates: flat $50 \mathrm{~nm}$ Au films on glass are readily available, since they are needed for the well established technique of SPRS. The latter is an analytical tool 
used in biology, that relies on the Kretschmann configuration. The exact same substrates can of course be used for SERS experiments.

- Uniform enhancement on the surface: since a flat surface has perfect translational symmetry (in the absence of roughness), there is no gradient for the LFEF on the surface. To obtain a uniform signal the only remaining challenge is the deposition of a uniform layer of molecules with known density. Langmuir Blodgett films [98] are just one of a number of options to achieve just that.

- Good theoretical understanding: for many of the application in SERS a good theoretical understanding of the LFEF on the substrate is imperative. For flat surfaces analytical expressions (Fresnel equations) as well as numerical codes (like the SPlaC code [2]) are readily available.

- Ideal platform for fundamental studies: because of its simplicity and well understood behavior, a flat metallic surface is the ideal platform to explore fundamental questions e.g. about surface selection rules (such as in Chapter3) and chemical enhancement [99].

Seeing these advantages, it is somewhat puzzling that flat metallic surfaces have so far not made it into the mainstream of SERS substrates. This, however, is likely to be attributed to two major facts:

- Firstly, the KC in its canonical setup (i.e. a prism attached to a glass slide with a thin (50nm) $\mathrm{Au}$ film) is rather cumbersome, making it difficult to focus on the surface and thereby prohibiting to perform microscopy on the sample. By showing (in Section 4.5 of this chapter) that it is possible to perform SPRS concomitantly with SERS, we hope that the cost-benefit ratio of using this setup will change for the better.

- Secondly, the KC as it was originally published in 1968 [71] "wastes" a lot of the available signal: it only captures a fraction of the so called Kretschmann cone. A design has been proposed [100] that allows with the help of a so called "Weierstrass-prism" just that - but it is even more cumbersome than before and still allows no microscopy. By proposing a new, simple setup, that allows one to capture all of the signal and perform microscopy, we hope not only to introduce a platform for fundamental studies but also to effect a change in paradigm toward more simple SERS substrates.

\subsubsection{Historical background}

A short historical background is necessary here to put this chapter's results and efforts into perspective. SERS in the KC is not a new idea. It was predicted theoretically in 1976 [101] even before the explanation of the SERS effect in 1977 [6] and was investigated experimentally from the early days of SERS. These early experiments were carried out on rough Ag films [102-104]. In fact, no SERS signal could be observed before the metallic film was roughened electrochemically $[102,104]$. It is well known that roughness is an important factor contributing to the SERS EF and that rough metallic films are simple SERS substrates with reasonable average SERS EF. Roughness however defeats the purpose of uniform enhancements that flat surfaces promise. In investigating SERS in the KC, it is therefore critical to assess whether or not roughness contributes to the SERS EF. This is precisely one of the novel contribution of our work. In this context, these early works 
$[102,104]$ on surface roughening demonstrated one important aspect of the problem of SERS in the KC: the observation of the SPP resonance (i.e. resonant angular dependence of the reflectivity and/or SERS signal) is not in itself a proof that roughness does not contribute to the SERS EF.

Following these, SERS in the KC with Ag films was carefully and much more convincingly studied, both experimentally and theoretically, in a series of papers in the 1980s [105-108]. Giergiel et al. provide a detailed summary of the findings up to 1988 in Ref. [108]. The additional benefit of collecting the light on the prism side and the peculiar emission pattern in the form of a Kretchmann cone [106] were clearly identified. SERS signals from both liquids [105-107] and adsorbates $[106,108]$ were successfully measured and their expected angular dependences (in the form of SPP resonances in incident or scattered light) were observed. Although care was taken in fabricating smooth films, the absence of contribution of any residual roughness to SERS was not demonstrated. Two attempts to estimate the SERS EF were made, but with no clear conclusion. In one case [108], the measured EF was unexpectedly small $(\approx 5)$. In the other [105], it was a factor of 10 too large ${ }^{1}$, which would suggest that roughness was here contributing significantly to SERS.

Then, most reports of SERS in the KC in the 1990s focused on a new technical development of the technique to allow one to collect the whole Kretchmann cone on the prism side, using either mirrors [109] or a so-called Weierstrass prism [100, 110-112]. Some of these studies used the Otto configuration [110-112] and reported measurements of SERS EFs for relatively thick adsorbed layers (a few nanometers). In this case however, the adsorbates strongly affect the SPP properties and therefore do not reflect the true SERS EF of the metal films. Moreover, roughness was again not excluded and was even present according to the authors [112].

Over the last decades, reports of SERS in the KC have not focused on the understanding of the method, but mostly on its practical use in research as a characterization tool, for example to determine molecular orientations on the surface $[113,114]$. Such a move toward applications of the techniques further emphasize the need to complete our fundamental understanding of the underlying mechanisms. We also note a recent report [115] of SERS in the KC where light is collected on the air side (even if prism-side has been shown to be a better option) and where a SERS EF of over $10^{6}$ is measured. Such a large EF can only be explained, once again, by roughness.

This brief review of the existing work on SERS in the KC highlights a number of outstanding issue:

- Can we demonstrate that SERS in the KC is possible, ideally from no more than a monolayer of adsorbate, in the absence of any roughness contribution to the SERS EF?

- Since all the cited studies but two $[112,114]$ were carried out on Ag films, can this method be convincingly extended to Au films, which are a better alternative for applications, and are already extensively used in SPRS instruments.

- can we demonstrate a simple optical layout for SERS in the $\mathrm{KC}$, which would be readily adaptable to SPRS?

- can we demonstrate a simple optical layout to collect the whole Kretschmann cone?

- and finally: how does our experimental data compare to theory?

\footnotetext{
${ }^{1}$ According to the calculation at the end of Ref. [105], the EF was $4 \times 10^{4}$, in agreement with theory. However, if one repeats the simple EF calculation with the parameters provided in the paper, the obtained value is 10 times larger, which suggests a calculation error by the authors.
} 
We therefore revisit in this chapter the problem of SERS in the KC with a view to address these outstanding issues in its connection with SPRS. We shall show that under the right conditions the KC offers a valid alternative for a reliable and homogeneous SERS enhancement that can be combined with SPRS simultaneously. We will show that such measurements can, moreover, be reliably understood within standard electro-magnetic theory with a minimum number of parameters: a tremendous advantage over most SERS systems. We shall also introduce a simple method to measure SERS in backscattering in the Kretschmann configuration using back focal plane illumination to improve coupling to the resonance and combine a high angular resolution with a good collection efficiency. Our method requires minimal changes to standard Raman systems to adapt them to the simultaneous use of SERS and SPRS. We believe that the combination of the two techniques, with the predicting power displayed in the following sections, can lead to a substantial progress in analytical applications where quantification and detailed understanding is essential. 


\subsection{Theory}

The analysis of the historical background in the previous section allowed us to place the KC in its historical context and to highlight pending questions and issues that still need to be resolved (e.g. confirming the absence of surface roughness). In this section a brief summary of the theory underlying the excitation of SPPs shall be given which has already been discuss in part in Section 1.4 and can be found in further detail in numerous textbooks $[2,17,18]$.

\subsubsection{Excitation of SPPs on flat surfaces - the resonance condition}

The KC [71] constitutes a canonical example of SPPs at a planar interface that has been treated and analyzed in many textbooks $[2,17,18]$ together with the associated Otto configuration [116]. Therefore, we shall only highlight a few key aspects here as background material. SPPs are solutions of Maxwell's equations at angular frequency $\omega$ localized at the interface between a metal (with dielectric function $\epsilon_{\mathrm{m}}(\omega)$ ), and a dielectric medium (with dielectric function $\epsilon_{\mathrm{d}}(\omega)$ ). The solution is characterized by an amplitude decaying exponentially away from the interface, and a propagating character along it (only limited by the inevitable residual losses). Ignoring the "small" imaginary part of the dielectric function of the metal for the time being [2] - i.e. considering both $\epsilon_{\mathrm{m}}(\omega)$ and $\epsilon_{\mathrm{d}}(\omega)$ to be real - it can be shown by matching boundary conditions in Maxwell's equations that localized SPPs at the interface can only exists if both conditions a) $\epsilon_{\mathrm{m}}(\omega) \cdot \epsilon_{\mathrm{d}}(\omega)<0$ and b) $\epsilon_{\mathrm{m}}(\omega)+\epsilon_{\mathrm{d}}(\omega)<0$, are satisfied simultaneously. This situation is attainable at a metal/dielectric interface, where we would normally have $\epsilon_{\mathrm{m}}(\omega)$ larger than $\epsilon_{\mathrm{d}}(\omega)$ in absolute value (but negative) over a large range of frequencies. Hence, if $k_{x}^{\mathrm{SPP}}$ is the SPP wavevector along the surface, light of frequency $\omega$ with a well defined dispersion relation

$$
k_{x}^{\mathrm{SPP}}=k_{0} \sqrt{\frac{\epsilon_{m}(\omega) \epsilon_{d}}{\epsilon_{m}(\omega)+\epsilon_{d}}} \text { with } k_{0} \equiv \frac{\omega}{c}
$$

can excite at the interface a SPP (a mixture of electromagnetic and surface-charge density wave $[2,17,18])$. In order to couple to SPPs with light we need to match both $\omega$ and $k$ with an incoming electromagnetic wave. This cannot be achieved by light propagating in free space, for the wavevector of light is always smaller than that of SPPs of the same frequency. But it can be accomplished by coupling to SPPs through an evanescent wave coming from a medium with a dielectric function $\epsilon_{\mathrm{g}}(\omega)$ larger than $\epsilon_{\mathrm{d}}(\omega)$. The dielectric medium at the interface will most often be air $\left(\epsilon_{\mathrm{d}}(\omega)=1\right)$ or water $\left(\epsilon_{\mathrm{d}}(\omega)=1.33\right)$, while the coupling medium is glass $\left(\epsilon_{\mathrm{g}}(\omega) \sim 1.5-1.7\right)$. The strategy of coupling through an evanescent wave is quite general and it typically comes in the form of the Kretschmann or Otto configurations. The evanescent field can be formally considered as a traveling wave (parallel to the surface) which can - for the right angle of incidence - "couple"' to the SPPs on the metal by transferring energy to the surface wave from the incoming light beam. The coupling can be observed as a dip in the reflected beam (Fig. 45) and this is a key application of SPPs in SPRS [64-68]. The fact that the energy of the wave is confined to the surface within a distance comparable to the wavelength (the evanescent field) results in a natural enhancement of the field at the surface. Intensity enhancement factors in the range $\sim 10-200$ (depending on the exact conditions) are normal, and are responsible for the surface sensitivity of SPRS. Small environmental changes at the surface affect the resonant coupling to SPPs and can be detected as changes in the reflectivity of the light beam. This is the basis of the entire field of SPRS, widely used in biology 
[110]. It is important to point out that only TM-waves can satisfy the right boundary conditions and are suitable for coupling to SPPs $[2,17,18]$.

\subsubsection{Dipole emission on a flat surface - the Kretschmann cone}

In the previous paragraph we have been able to show that it is possible to excite SPPs and noted the expression for the resonance condition at which this can be done (eq. 4.1). On a practical level the Kretschmann configuration provides a way to implement such an excitation (see Section 4.5 and 4.6). Once excited, those SPPs result in moderate LFEF $\left(\sim 10^{2}\right.$-fold for the perpendicular electric field component on $\mathrm{Au}$ ) that can be easily calculated using the SPLaC program [2]. As an example the perpendicular and parallel LFEF for TM-polarized light are given in Fig. 34 for a 50nm Au sample on glass at the Au/air interface.

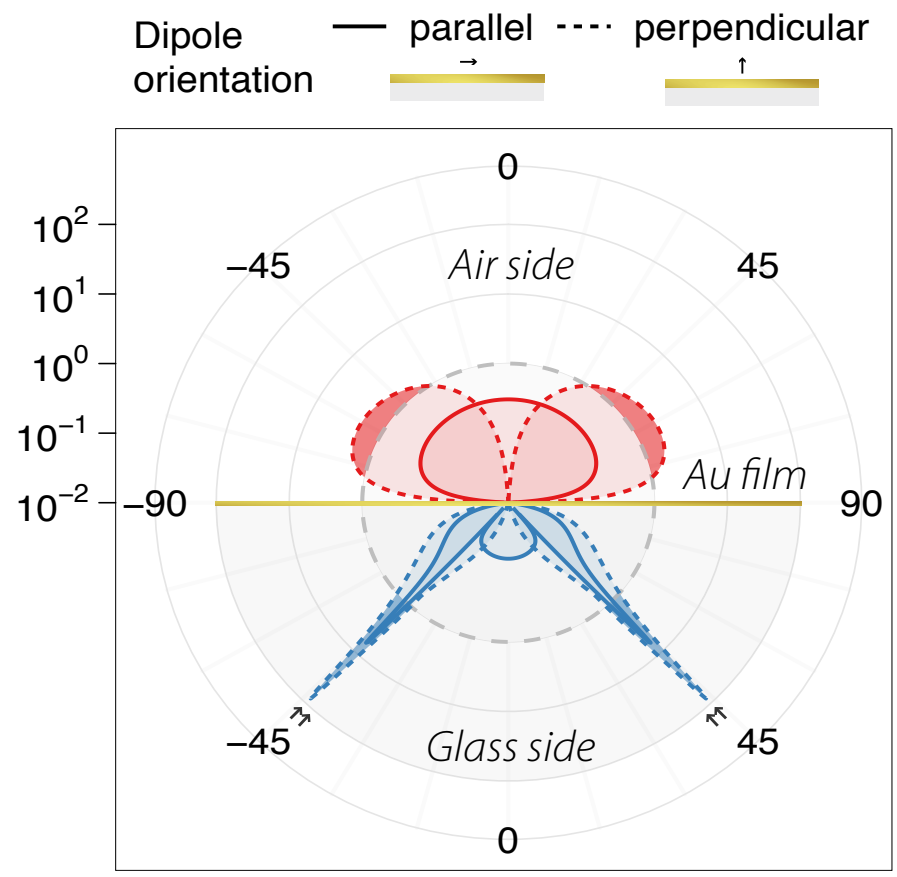

Fig. 34:

The theoretical LFEF (in log-polar coordinates) at $\lambda=632.8 \mathrm{~nm}$ for the parallel (solid lines) and perpendicular (dashed lines) electric field components at $1 \mathrm{~nm}$ above a $50 \mathrm{~nm}$ Au layer supported by a semi-infinite glass substrate $(n=1.52)$ and excited with TMpolarized light. By virtue of reciprocity this pattern also corresponds to the radiative enhancement (see Chapter 1) of a dipole on the surface; The critical angle of total internal reflection and the resonance angle $\theta_{K}$ for excitation of SPPs at the air/Au interface are indicated by small arrows $\left(41.1^{\circ}\right.$ and $43.5^{\circ}$, respectively).

While it is obvious that on the air side the fields are mostly quenched $(M<1)$, a remarkable effect can be witnessed on the glass side where both field components experience significant enhancements in a narrow angular region around the resonance angle $\theta_{K}$ (also called Kretschmann angle). Such enhancements of the electromagnetic field provided by the surface plasmon resonance (SPR) benefit the SERS signal in two ways. First, as the incident light couples to the resonance, the Raman probe experiences an enhanced excitation; second, by virtue of reciprocity (see Chapter 1), light emission at the Raman frequency is also enhanced in the same fashion. This is described more formally by the well known SERS EF $F$ that has been derived in Section 1.3 and has the form:

$$
F^{\mathbf{P}_{\mathrm{L}}-\mathbf{P}_{\mathrm{R}}}=\left|\hat{\mathbf{E}}_{\mathrm{loc}}^{\mathbf{P}_{\mathrm{R}}}\left(\lambda_{\mathrm{R}}, \theta_{\mathrm{R}}\right) \cdot \hat{\alpha}_{\mathrm{N}} \cdot \hat{\mathbf{E}}_{\text {loc }}^{\mathbf{P}_{\mathrm{L}}}\left(\lambda_{\mathrm{L}}, \theta_{\mathrm{L}}\right)\right|^{2}
$$

For the case of a uniaxial tensor $\hat{\alpha}$ oriented perpendicular or randomly parallel to the metal sur- 
face, this expression can be simplified (see Section 3.3):

$$
\begin{aligned}
& F_{\perp}^{\mathbf{P}_{\mathrm{L}}-\mathbf{P}_{\mathrm{R}}}=\frac{15}{4} M_{\perp}^{\mathbf{P}_{\mathrm{R}}}\left(\lambda_{\mathrm{R}}, \theta_{\mathrm{R}}\right) M_{\perp}^{\mathbf{P}_{\mathrm{L}}}\left(\lambda_{\mathrm{L}}, \theta_{\mathrm{L}}\right) \\
& F_{\|}^{\mathbf{P}_{\mathrm{L}}-\mathbf{P}_{\mathrm{R}}}=\frac{15}{4} \kappa^{\mathbf{P}_{\mathrm{L}}-\mathbf{P}_{\mathrm{R}}} M_{\|}^{\mathbf{P}_{\mathrm{L}}}\left(\lambda_{\mathrm{L}}, \theta_{\mathrm{L}}\right) M_{\|}^{\mathbf{P}_{\mathrm{R}}}\left(\lambda_{\mathrm{R}}, \theta_{\mathrm{R}}\right)
\end{aligned}
$$

Apparently $F$ is invariant with respect to rotations around the surface normal, a fact that reflects the symmetry of the problem (uniaxial tensor on a flat Au surface). Because the resonant coupling

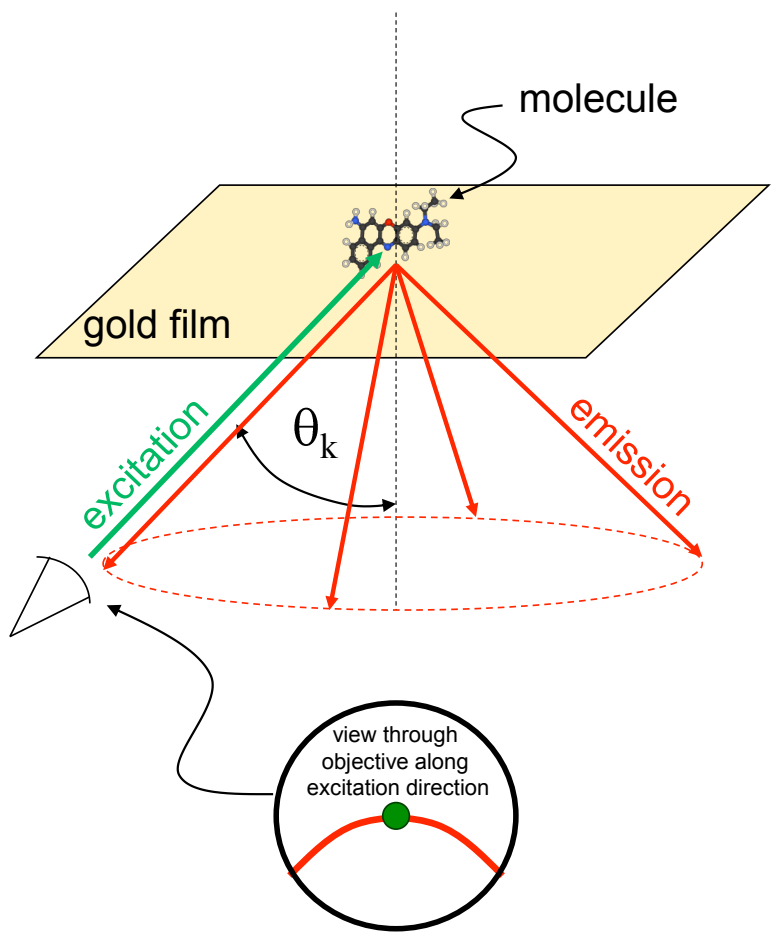

Fig. 35 : A molecule is excited at the Kretschmann angle $\theta_{K}$ (with TM-polarization) and the Ramanshifted light is emitted at a very similar angle, but in any direction, i.e. in the Kretschmann cone. Light collected in backscattering by an objective with numerical aperture NA contains only a fraction of the Kretschmann cone. to SPPs (as described by $M^{\mathbf{P}_{\mathrm{L}}}$ in eq. 4.4 and 4.3 ) is relatively sharp in incident angle (see Fig. 34) but broad in the spectral domain, the emission (as described by $M^{\mathbf{P}_{\mathrm{R}}}$ ) at the Raman-shifted frequency occurs similarly over a narrow angle around $\theta_{K}$. In principle $\theta_{K}$ for emission is different to that of the incident coupling because of the different wavelength introduced by the Stokes shift. This may be an issue in some cases (and it is explicitly taken into account in the theoretical predictions), but in a first approximation to the problem the change in angle may be neglected; especially for relatively small Raman shifts.

For the case of TM-excitationpolarization and a TM-emissionfilter, one can envision this radiation pattern on the glass side as a very narrow cone (called the Kretschmann cone) that contains most of the emitted signal and is schematically depicted in Fig. 35. It is quite obvious that EF of up to $\sim 10^{4}$ are possible for molecules oriented perpendicular to the surface.

Part of the challenge of combining SPRS with SERS is in the simultaneous optimization of both the excitation of SPPs and the collection of the Kretschmann cone.

\subsubsection{Emission profile in the Kretschmann configuration}

In Section 3.3 we were able to derive an expression (eq. 3.28) for the power $P$ emitted from a monolayer of uni-axial molecules of density $\rho$ from a flat surface that required only the knowledge of the 
LFEF in excitation $M^{\mathbf{P}_{\mathrm{L}}}$ and emission $M^{\mathbf{P}_{\mathrm{L}}}$ :

$$
P_{\mathrm{Rad}}^{\rho}(\alpha)=\rho \cdot \frac{\mathrm{d} \sigma}{\mathrm{d} \Omega} \cdot K_{\#} \cdot \underbrace{\int_{E\left(\alpha, N A_{\text {Las }}\right)}\left(\frac{1}{\cos (\alpha)} M_{\#}^{\mathbf{P}_{\mathrm{L}}}\left(\Omega^{\prime}\right) \cdot \frac{\mathrm{d} P^{\mathrm{inc}}}{\mathrm{d} \Omega}\left(\Omega^{\prime}\right)\right) \mathrm{d} \Omega^{\prime}}_{\text {excitation }} \cdot \underbrace{\int_{D\left(\alpha, N A_{\mathrm{Obj}}\right)} M_{\#}^{\mathbf{P}_{\mathrm{R}}}\left(\Omega^{\prime}\right) \mathrm{d} \Omega^{\prime}}_{\text {emission }}
$$

Here $K \equiv \frac{15}{4} \cdot \kappa_{\#}^{\mathbf{P}_{\mathrm{L}}-\mathbf{P}_{\mathrm{R}}}$ (the values for $\kappa_{\|}^{\mathbf{P}_{\mathrm{L}}-\mathbf{P}_{\mathrm{R}}}$ are summarized in Table 3.24 and $\kappa_{\perp}=1$ ).

It is easy to see that this expression still holds true for the Kretschmann configuration, even when the signal is collected from the glass side. We only need to make sure to use the right $M$-values (i.e. the $M$ values for the glass side instead of the air side as plotted in Fig. 34). With this in mind and with the definitions of the set of points $E$ and $D$ (for excitation and detection, see eq. 3.30 and 3.29) we can evaluate this expression numerically as described in Section 3.3.4. Care needs to be taken when choosing the grid points: since $M$ varies much more rapidly on the glass side than it does on the air side, it becomes necessary to have more grid/points per solid angle.

\subsubsection{EF calculations - a guide for an intuitive understanding}

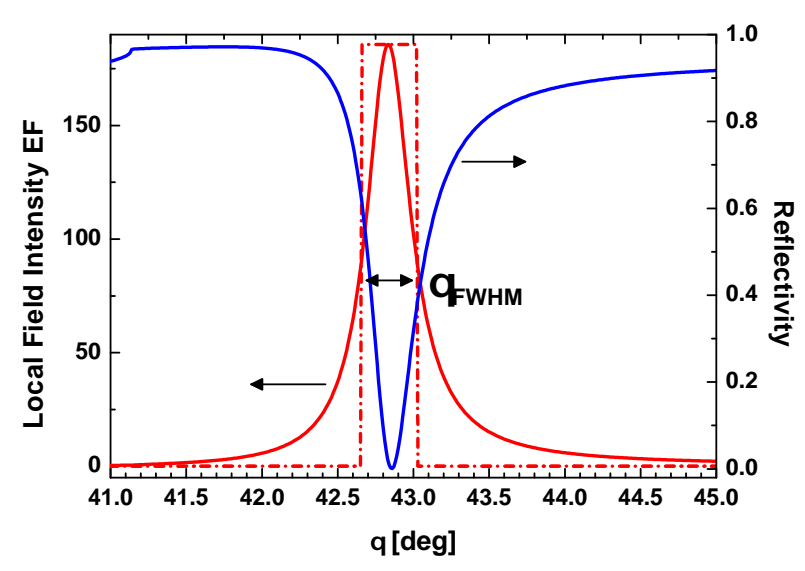

Fig. 36 : $L F E F$ (red) and reflectivity (blue) for a $50 \mathrm{~nm} \mathrm{Au}$ film on glass. Note the strong correlation in width of the blue and red curve.
It is easy to see that the exact evaluation of the excitation and emission integrals in eq. 4.5 can be lengthy and cumbersome. For this reason, and in order to give the reader a more intuitive understanding of the peculiar emission profile at hand, we would like to show here a way, how one can estimate both of these integrals only by knowing the reflectivity $R$ and the LFEF $M$ as a function of angle. $R$ and $M$ are easily (and very quickly) obtained with the help of the SPLaC program (see [2], Appendix F).

Firstly our goal is to estimate the emission-integral in eq. 4.5. In order to do so, an effective solid angle $\Delta \Omega_{\text {eff }}$ is defined by integrating over the objective's "field of view" as characterized by $D\left(\alpha, N A_{\mathrm{Obj}}\right)$ :

$$
\int_{D\left(\alpha, N A_{\mathrm{Obj}}\right)} M\left(\Omega^{\prime}\right) \mathrm{d} \Omega^{\prime}=: M_{\max } \cdot \Delta \Omega_{\mathrm{eff}}^{M}
$$

Clearly, $\Delta \Omega_{\text {eff }}^{M}$ is well defined but the question is, if there is a way to approximate its value without carrying out the numerical calculations required for the left hand side of the above equation. One possibility for such an approximation is to consider a simplified $M$, that has the shape of a step function of width $\theta_{\mathrm{FWHM}}$ (plotted as a dotted line in Fig. 36, which we shall call $\tilde{M}$ ). One may derive from Fig. 35 that for small solid angles $\gamma_{\mathrm{Obj}}$ the effective solid angle $\Delta \Omega_{\text {eff }}$ for $\tilde{M}$ amounts to:

$$
\Delta \Omega_{\mathrm{eff}}^{\tilde{M}} \approx 2 \gamma_{\mathrm{Obj}} \cdot \theta_{\mathrm{FWHM}}
$$


In this equation $\gamma_{\mathrm{Obj}}$ is the opening angle of the objective (see Chapter 3.3) and hence we can approximate $\Delta \Omega_{\text {eff }}^{M} \approx \Delta \Omega_{\text {eff }}^{\tilde{M}}$.

Secondly our goal is now to estimate the excitation integral in eq. 4.5.

$$
\int_{E\left(\alpha, N A_{\text {Las }}\right)} \frac{1}{\cos (\alpha)} M\left(\Omega^{\prime}\right) \frac{\mathrm{d} P^{\text {inc }}}{\mathrm{d} \Omega}\left(\Omega^{\prime}\right) \mathrm{d} \Omega^{\prime}=: M_{\text {max }} \cdot P_{\text {eff }}^{\text {inc }}
$$

Here $P_{\text {eff }}^{\text {inc }}$ is again defined implicitly. One way to estimate $P_{\text {eff }}^{\text {inc }}$ is by simply approximating it with the absorbed power $P^{\text {abs }}$ which can be determined experimentally or numerically.

While the approximations described here allow us to obtain an intuitive understanding of the situation and a reasonable estimate of the maximum enhancement factor at resonance, there are certain things that are not accounted for with those simple approximations:

- Laser induced broadening of resonance: for excitation and emission along a single direction we expect the Raman signal to follow $M^{2}(\theta)$ as we scan through the resonance. Even when collecting the signal with an objective we still would expect a profile proportional to $M(\theta)$. However, experimentally we observe a broadened resonance that we can easily attribute to the angular spread in excitation. In order to predict the exact shape of this broadened resonance we need to carry out the numerical calculations.

- Raman induced shift of the emission profile: For a small Raman shift the approximation $M\left(\lambda_{\mathrm{L}}\right)=M\left(\lambda_{\mathrm{R}}\right)$ may be permissible. However, as we observe the resonance of higher and higher Raman shifts this approximations becomes more and more inaccurate as the maximum of excitation and emission resonance profile no longer coincide which broadens the resonance even further.

- Distortion of excitation at large angles: It can be shown that for a small angle $\beta$ between the optical axis and the normal of the prism, the distortion of the angular power distribution $P_{\text {rad }}^{\rho}(\theta)$ is negligible. However, as the angles increase (e.g. for a saphire/Au/water interface) the associated change in the resonance profile can no longer be ignored. 


\subsection{Experimental setup}

In the previous section the existence of a SPP at a metal-air interface was shown. From its resonance condition it became apparent, that $k_{x}^{\mathrm{SPP}}>k_{0}$ and that it is therefore impossible to excite such a SPP with a propagating wave from the air side (i.e. simply shining light on the interface from the air side will not do the trick). Instead one has to couple to this surface wave by using a special experimental setup like the Kretschmann- or Otto configuration. In both setups the k-vector of the excitation light source is first "boosted" by means of a high index of refraction material (usually a glass prism) and then delivered as an evanescent field to the air/metal interface through a very thin intermediate layer. In the Otto configuration this intermediate layer is a thin gap of air that separates the prism from the metal surface. In the Kretschmann configuration the intermediate layer consists of $50 \mathrm{~nm}$ of metal.

For the work presented in this chapter we focus on the KC. While the general idea of this setup is simple and based on SPP-excitation by illumination of a 50nm Au film from the glass side, there are many ways to implement this idea. Here we would like to give a systematic overview of the different available setups, explaining their pros and cons in terms of SPP excitation efficiency, SERS detection capacity and SPRS compatibility. This will prepare the ground to discuss the work done in two of these setups in more detail.

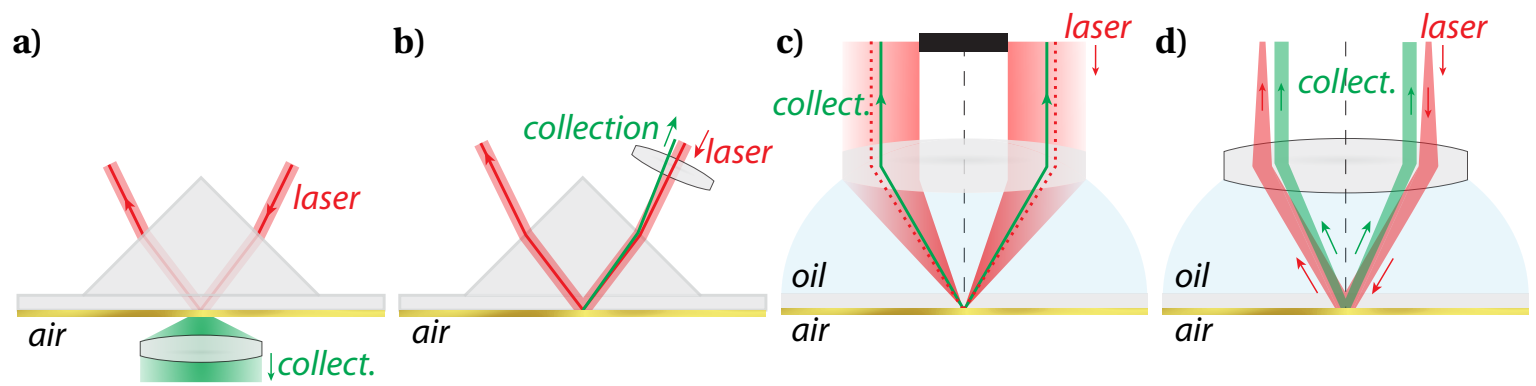

Fig. 37 : Diagrams of experimental schemes combining SPRS and SERS in the KC. The laser light is represented in red, the Raman-shifted radiation in green. a) Prism coupling of a laser beam, and collection of the Raman-scattered light from the air side [115]. b) Prism coupling, and collection from the prism side using a long-distance low-to-medium NA objective [60]. c) The incident beam is expanded to fill the objective, and the central part of the illumination cone is blocked [117]. The Raman signal is collected in back-scattering from the same objective. d) A shifted laser beam traverses off-axis a high NA oil-immersion objective, and illuminates the Au film as a parallel beam. The Raman signal is collected through the same objective. In $b-d$ ) the minute angular shift between excitation and collection produced by the dispersion of the resonance (typically $\sim 1^{\circ}$ ) is exaggerated for clarity.

General remarks: Because of the sharp angular dependence of the coupling to SPPs, the excitation and collection of SERS signals in the KC require a number of special considerations compared to "standard" SERS experiments. In particular, efficient coupling to SPPs in excitation requires a highly collimated (non-divergent) exciting beam [60]. Moreover, the SERS signal on the prism side is primarily emitted within a narrow angular cone: the Kretschmann cone. These two major aspects are rooted in the peculiar angular shape of the LFEF shown in Fig. 34 and are schematically illustrated in Fig. 35. Because of the remarkable variation of $F \propto M_{\#}^{\mathbf{P}_{\mathbf{L}}} \cdot M_{\#}^{\mathbf{P}_{\mathrm{R}}}$ (see eqs. 4.3 and 4.4) 
as a function of angle (which stands in contrast to the vast majority of other SERS experiments) the actual enhancement of the SERS signal crucially depends on the excitation and collecting optics (i.e. whether the full Kretschmann cone is being collected, whether the excitation beam is fully coupled to the SPPs, etc). The signal enhancement in this configuration, accordingly, cannot be fully summarized as a single number, but rather it needs to be accompanied by a detailed specification of the excitation and collection optics configuration, which shall be discussed in the following. Four possible schemes are presented in Fig. 37 which allow simultaneous SPP excitation and collection of SERS signals.

Setup A: Perhaps the most natural extension to the original Kretschmann scheme is shown in Fig. 37a) [115]. A laser beam couples to SPPs using the original Kretschmann excitation scheme with a glass prism. The SERS signal is collected from the air side with an objective in close proximity to the surface [115]. In this excitation scheme the collection of the Raman signal from the air side misses the additional enhancement mechanism of the scattering process in the Kretschmann cone, situated on the opposite side. Even though this can be partially compensated by using a high NA objective (the total signal, integrated over all angles, from the front is comparable to the total signal from the glass side), a major difficulty in this non-conventional excitation/collection configuration remains in the alignment of the excitation spot with the collection objective. Especially for doing microscopy and for combining SPRS with SERS (simultaneous measurement of the angular-dependent reflectivity and SERS-signal) this poses a significant obstacle.

Setup B: In contrast, the schematic proposed in Fig. 37b) collects the SERS signal from the prism side, in the more standard and much more convenient back-scattering configuration [60], that makes alignment of excitation and emission (as required in setup A) unnecessary. Even though it is easy to setup and compatible with most spectrometers, this excitation scheme precludes tight focusing as the physical presence of the prism limits the use of high- $N A$ objectives. Furthermore only a small fraction of the Kretschmann cone of emitted Raman radiation is collected (as illustrated in Fig. 35). As in the previous setup A, microscopy is rather cumbersome to do because the sample's surface normal and the objective's axis are at an angle.

Setup C: The setup presented in Fig. 37(c) - also based on a backscattering configuration - is more suitable for microscopy, using a high-numerical aperture oil-immersion objective in place of the glass prism to reach the angle of incidence corresponding to the excitation of SPPs. It is based on Ref. [117], where such an approach was used for fluorescence, but not SERS, measurements: an expanded laser beam fills the back aperture of the objective and is tightly focused onto the substrate. A (small) portion of the beam couples to SPPs, and the SERS signal is collected in backscattering using the same objective. To restrict the range of incident angles, and avoid undesirable heating of the metal substrate at high incident power, the central portion of the incident beam may be blocked. This setup has the problem that the spread of incident angles for coupling cannot be controlled very well, and the resulting poor coupling to SPPs prevents the simultaneous measurement of the SPRS reflectivity (it also dramatically reduces SERS intensities, but this could in principle be compensated by a higher laser power).

Setup D: Finally, Fig. 37d) depicts a new scheme proposed in this thesis, which is similar to C and to the scheme used for SPRS microscopy [118]. It can be seen as a hybrid setup between those in Figs. 37b) and c), where an off-center incident beam enters a high- $N A$ oil-immersion objective, which is also used for efficient collection of the SERS signals. This approach presents several advantages, including: $(i)$ it is based on the convenient back-scattering configuration and can therefore be implemented in most Raman microscopes with only minor modifications. ( $i i$ ) The 
high- $N A$ objective in principle allows for a high spatial resolution for either spectroscopy or imaging of the collected light. ( $\mathrm{i}$ i ) The entire Kretschmann cone is collected, thereby maximizing the SERS signal. ( $i v$ ) As will be shown in this work, it is possible under appropriate conditions to couple efficiently to SPPs and therefore carry out simultaneous measurements of the SPR reflectivity. $(v)$ It is possible to do microscopy in this setup.

Other Approaches: It is worth noting that by using a much more complicated optical setup, with special prisms and/or optics (like the Weierstrass prism [100, 110-112]), one can devise ways to collect the entire Kretschmann cone [100, 109]. This was certainly an important improvement in the historical development of both the Kretschmann and Otto configurations. But it comes at the expense of complexity with a customized optical setup with independent excitation and collection directions. In fact, this is the main reason why the KC has not found widespread use in SERS. It also makes it difficult to simultaneously measure the angle-dependent reflectivity. 


\subsection{Combining SPRS with SERS - the prism setup}

In the previous section different approaches to implement the Kretschmann configuration were discussed. One of them (the prism setup in the backscattering configuration as shown in Fig. 37b) was used to obtain the data presented in this section. Those results allow us to show the possibility of combining SPRS with SERS. Furthermore theoretical predictions based on standard codes for planar multilayers (see SPLaC codes in Appendix F of reference [2]) will be presented on the same graphs as the experimental data to demonstrate the very good theoretical understanding attainable for flat metallic surfaces.

\subsubsection{The physical setup}

The prism and film are attached to a high-precision motorized rotating stage as shown in Fig. 38. The coupling between the substrate and the prism is achieved by optical oil (for microscopy). A $90^{\circ}$ optical coupler adapts the head of the Raman microscope to direct and collect the light from the Kretschmann set up either with or without an objective on the way. The far field images of the coupling to the resonance (fringe) can be seen in reflection over a white screen from the other side

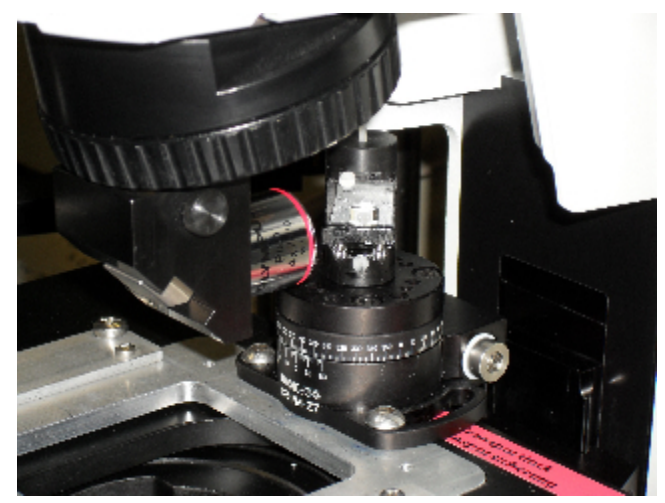

Fig. 38 : Adaptation of the Raman microscope for the Kretschmann configuration. of the prism. The adapted head of the microscope couples the signal detection to a triple additive/subtractive Jobin Yvon T64000 spectrometer equipped with a nitrogen cooled CCD detector (Symphony) and 600 lines/mm gratings (see Chapter 1). When using the BFP-lens, we attach the lens in the delivering optical path of the laser before the internal beamsplitter of the microscope. For metal/water interfaces we used a sapphire prism $(n=1.77)$, while all measurements in metal/air interfaces are done with a glass prism $(n=1.52)$. For the measurements on a metal/water interface, we designed a small cell that contains the water and can be attached to the film.

\subsubsection{Sample preparation}

For all experimental results, we have used NB as a model SERS analyte since it has a large (resonant) Raman cross-section at the excitation wavelengths used here (633 and $647 \mathrm{~nm}$ ) and can be deposited easily on metal surfaces at monolayer or sub-monolayer coverage by dipping/rinsing techniques.

Ag films $(50 \mathrm{~nm})$ were evaporated on clean glass slides using standard evaporation techniques. Au films (50 nm nominal thickness) grown on a $2 \mathrm{~nm}$ titanium adhesion layer were bought from SSens, Netherlands (with a standard RMS value of $1 \mathrm{~nm}$ on $1 \mu \mathrm{m}$ for the roughness). Films were dipped in a $100 \mu \mathrm{M}$ NB solution in water for $5 \mathrm{~min}(\mathrm{Ag}) / 40 \mathrm{~min}(\mathrm{Au})$, and then thoroughly rinsed with water. Ultra-pure water was used for sample preparation, as otherwise impurity islands form 
on the films and substantially diminish the signal homogeneity. The films are cut to the appropriate size when needed and attached to the coupling prism through an oil layer to avoid a discontinuity in the index of refraction. The homogeneity of the coverage is paramount if meaningful results are sought. We checked different preparation methods and assessed the homogeneity of the samples through Raman maps in different configurations. The surface coverage of NB was also estimated ( $\sim$ one monolayer) via electrochemical methods using the oxidation/reduction cycle of NB.

For the preparation of the liquid cell we used a procedure described in Fig. 39, with a series of pictures. From left to right: the Au sample is carefully placed on an aluminium substrate holder (picture 1) and the sapphire prism is attached to the glass side (picture 2). Now the sample is turned arround and a drop of dye-solution is placed on the Au side while a silicon o-ring is brought in place (picture 3). Finally a glass lid is placed on top and hold in place with 2 screws. This defines then a liquid layer above the metal that can be used for the studies.

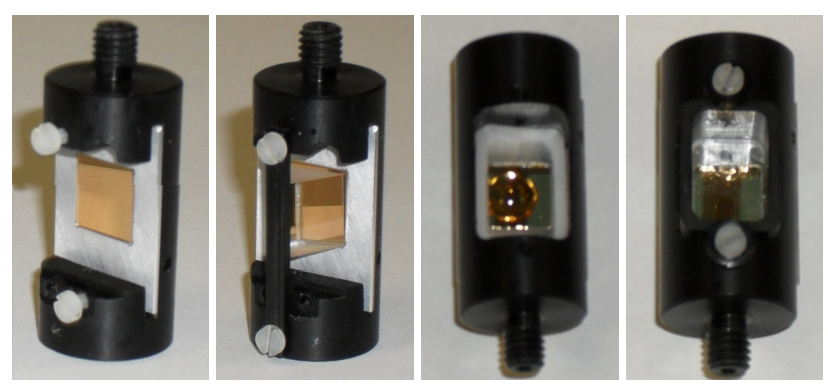

Fig. 39 :

Preparation of the liquid cell; from left to right: Au substrate in sample holder; sapphire prism on glass side (of Au substrate); drop of liquid on Au side of substrate; the sealed-off cell.

\subsubsection{Angle of incidence as a function of sample rotation}

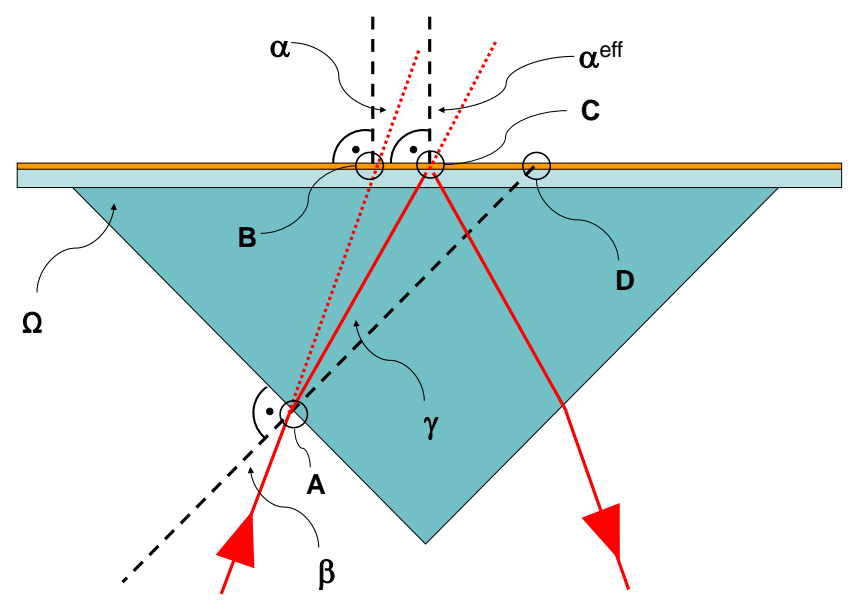

Fig. 40 :

Schematic illustration of a thin Au film attached to a prism. It shows the variables necessary to relate the internal angle of incidence $\alpha^{\text {eff }}$ to the external one $\alpha$. If the index of refraction $n_{\text {prism }}$ and its shape (characterized by $\Omega$ ) are known, $\alpha^{\text {eff }}$ can be obtained from $\alpha$ by simple trigonometry and Snell's law.

Experimentally, we measure for our incoming laser the angle of incidence $\alpha$ with respect to the normal of the sample surface (Fig. 40). However from a theoretical point of view (numerical calculation of the resonance angle etc.) we are more interested in the internal angle $\theta$ at the glass / metal interface (all our metal films are deposited on glass). Knowing the index of refraction of the glass-slide $n_{\text {glass }}$, of the prism $n_{\text {prism }}$ and the prism's shape (characterized by the angle $\Omega$ in Fig. 40) it is possible to establish a link between $\alpha$ and $\theta$. 


\section{case 1: glass-prism}

Using Fig. 40 and Snell's law the following equations are apparent:

$$
\begin{array}{ll}
\mathrm{ABD}: & \underbrace{\beta}_{\mathrm{A}}+\underbrace{\left(\alpha+90^{0}\right)}_{\mathrm{B}}+\underbrace{\left(180^{\circ}-90^{\circ}-\Omega\right)}_{\mathrm{D}}=180^{\circ} \\
\text { Snell : } & n_{\mathrm{air}} \sin \beta=n_{\text {prism }} \sin \gamma \\
\mathrm{ACD}: & \underbrace{\gamma}_{\mathrm{A}}+\underbrace{\left(\theta+90^{\circ}\right)}_{\mathrm{C}}+\underbrace{\left(180^{\circ}-90^{\circ}-\Omega\right)}_{\mathrm{D}}=180^{\circ},
\end{array}
$$

This equation can be rewritten as follows:

$$
\begin{aligned}
\text { eq. } 4.9 \Rightarrow \beta & =\Omega-\alpha \\
\text { eq. } 4.10 \Rightarrow \gamma & =\arcsin \left(\frac{n_{\text {air }}}{n_{\text {prism }}} \sin \beta\right) \\
\text { eq. } 4.11 \Rightarrow \alpha^{\text {eff }} & =\Omega-\gamma .
\end{aligned}
$$

Using eq. 4.12 and eq. 4.13 in eq. 4.14 yields:

$$
\alpha^{\text {eff }}=\Omega-\arcsin \left(\frac{n_{\text {air }}}{n_{\text {prism }}} \sin (\Omega-\alpha)\right)
$$

This last equation relates the angle $\alpha$ controlled with and displayed by the motorized stage to the angle of incidence $\theta$ on the metal film ${ }^{2}$. Throughout the figures in this chapter the $\mathrm{x}$-axis for angular scans refers to this angle of incidence $\alpha^{\text {eff }}$ on the film.

\section{case 2: sapphire-prism}

For the case of a sapphire prism the situation is slightly more complicated: the beam is refracted not only at the air / prism interface (as in case 1) but also at the prism / glass-slide interface. At this latter interface let us call the angle of incidence $\tilde{\alpha}^{\text {eff }}$. Then eq. 4.15 is obviously valid for $\tilde{\alpha}^{\text {eff. }}$

$$
\tilde{\alpha}^{\text {eff }}=\Omega-\arcsin \left(\frac{n_{\text {air }}}{n_{\text {prism }}} \sin (\Omega-\alpha)\right)
$$

Once more we know from Snell's law that:

$$
\begin{aligned}
n_{\text {prism }} \sin \tilde{\alpha}^{\text {eff }} & =n_{\text {glass }} \sin \alpha^{\text {eff }} \\
\Rightarrow \tilde{\alpha}^{\text {eff }} & =\arcsin \left(\frac{n_{\text {glass }}}{n_{\text {prism }}} \sin \alpha^{\text {eff }}\right)
\end{aligned}
$$

Inserting now eq. 4.16 into eq. 4.17 yields:

$$
\alpha^{\text {eff }}=\arcsin \left\{\frac{n_{\text {prism }}}{n_{\text {glass }}} \cdot \sin \left(\Omega-\arcsin \left(\frac{n_{\text {air }}}{n_{\text {prism }}} \sin (\Omega-\alpha)\right)\right)\right\}
$$

\footnotetext{
${ }^{2}$ The implicit assumption in this calculation is that the glass-slide's index of refraction is the same as the prism's index of refraction
} 


\subsubsection{Modified emission profile for a monolayer of molecules in the prism setup}

As has been discussed in more detail in the theory part of this chapter, any data taken in the KC on a thin Au film with a monolayer of molecules can still be fitted numerically using using eq. 4.5 from Chapter 4.3:

$$
P_{\mathrm{Rad}}^{\rho}(\alpha)=\rho \cdot \frac{\mathrm{d} \sigma}{\mathrm{d} \Omega} \cdot K_{\#} \cdot \underbrace{\int_{E\left(\alpha, N A_{\text {Las }}\right)}\left(\frac{1}{\cos (\alpha)} M_{\#}^{\mathbf{P}_{\mathrm{L}}}\left(\Omega^{\prime}\right) \cdot \frac{\mathrm{d} P^{\mathrm{inc}}}{\mathrm{d} \Omega}\left(\Omega^{\prime}\right)\right) \mathrm{d} \Omega^{\prime}}_{\text {excitation }} \cdot \underbrace{\int_{D\left(\alpha, N A_{0 \mathrm{obj}}\right)} M_{\#}^{\mathbf{P}_{\mathrm{R}}}\left(\Omega^{\prime}\right) \mathrm{d} \Omega^{\prime}}_{\text {emission }}
$$

Here $K \equiv \frac{15}{4} \cdot \kappa_{\#}^{\mathbf{P}_{\mathrm{L}}-\mathbf{P}_{\mathrm{R}}}$ (the values for $\kappa_{\|}^{\mathbf{P}_{\mathrm{L}}-\mathbf{P}_{\mathrm{R}}}$ are summarized in Tab. 3.24 and $\kappa_{\perp}=1$ ). It is quite easy to see that with the setups $C$ and $D$ displayed in Fig. 37 this equation is still accurate. For numerical calculations we only need to make sure to use the correct $M$-values (for the glass side instead of

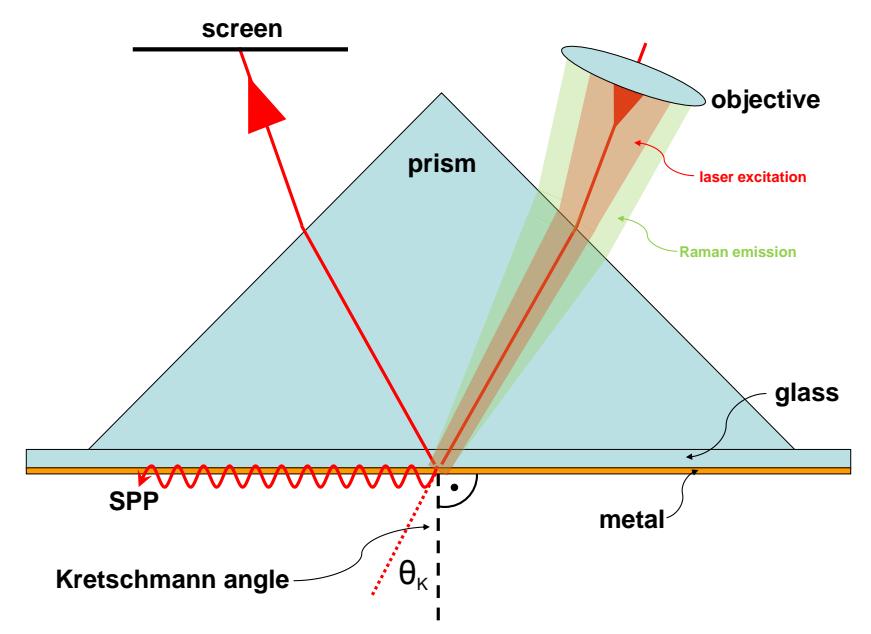

Fig. 41 : Closeup of the excitation-emission scheme in the Kretschmann configuration (setup A in Fig.3.32) showing refraction at the prism interface. the air side (Fig. 34)) and to choose more grid-points in a given angular range as the variation of $M$ with $\theta$ is much more rapid on the glass side. However, for the setups A and B in Fig. 37 (i.e. for the setups that involve the use of a prism) the situation gets slightly more complicated as can be appreciated from Fig. 41. Here the incoming light is refracted at the air-prism interface causing a change in direction (previously not taken into account) which modifies the effective NA of the lens.

In consequence the previous definition of the sets of points $E$ and $D$ (for excitation and emission, see eqs. 3.29 and 3.30) need to be modified:

$$
\begin{aligned}
D\left(\alpha, N A_{\mathrm{Obj}}\right) & \equiv\left\{\Omega: \tilde{\theta} \leq \gamma_{\mathrm{Obj}}\right\} \\
E\left(\alpha, N A_{\text {Las }}\right) & \equiv\left\{\Omega: \tilde{\theta} \leq \gamma_{\text {Las }}\right\}
\end{aligned}
$$

1. As mentioned before, the local field enhancement factor $M$ in eq. 4.19 can be calculated numerically with the SPLaC program using the input parameters already mentioned in Chapter 3 ( $45 \mathrm{~nm}$ thick Au film on a $2 \mathrm{~nm}$ Ti adhesion layer on a semi-infinite glass slide): $M_{\mathrm{SPLaC}} \equiv$ $\left|\mathbf{E}_{\text {loc }}\right|^{2} /\left|\mathbf{E}_{\text {glass }}\right|^{2}$. In reality, of course, the glass layer is not semi-infinite and what we are really interested in is:

$$
M \equiv \frac{\left|\mathbf{E}_{\text {loc }}\right|^{2}}{\left|\mathbf{E}_{\text {air }}\right|^{2}}=\underbrace{\frac{\left|\mathbf{E}_{\text {glass }}\right|^{2}}{\left|\mathbf{E}_{\text {air }}\right|^{2}}}_{\mathrm{C}} \cdot \underbrace{\left|\mathbf{E}_{\text {loc }}\right|^{2}}_{M_{\text {SPLaC }}}
$$


The correction factor $C \equiv\left(E_{\mathrm{pr}} / E_{\mathrm{air}}\right)^{2}$ in eq. 4.22 relates the incoming to the transmitted electric field at the boundary of two dielectrics - a standard exercise shown in many basic textbooks on Electrodynamics. In [2], p.560, eq. F.56 this ratio is written for a TM polarized wave (which is the polarization relevant in the Kretschmann configuration):

$$
\begin{aligned}
C \equiv\left(\frac{E_{\mathrm{pr}}}{E_{\mathrm{air}}}\right)^{2} & \stackrel{(F .56)}{=} \frac{\epsilon_{\mathrm{air}}}{\epsilon_{\mathrm{pr}}}\left|t^{p}\right|^{2} \\
& \stackrel{(F .46)}{=} \frac{\epsilon_{\mathrm{air}}}{\epsilon_{\mathrm{pr}}}\left|\frac{2}{1+K^{p}}\right|^{2}
\end{aligned}
$$

Using the canonical definition $n \equiv \sqrt{\epsilon}$ and the definition for $K^{p}$ on page 559 of [2]:

$$
K^{p} \equiv \frac{n_{\mathrm{air}} \sqrt{1-\frac{n_{\mathrm{air}}^{2}}{n_{\mathrm{pr}}^{2}} \sin ^{2} \beta}}{n_{\mathrm{pr}} \cos \beta} \stackrel{\beta=0^{0}}{=} \frac{n_{\mathrm{air}}}{n_{\mathrm{pr}}}
$$

We can rewrite eq. 4.23 for $\alpha=0^{0}$ as:

$$
C=\left(\frac{2 n_{\text {air }}}{n_{\text {air }}+n_{\text {glass }}}\right)^{2}
$$

With a glass-prism $\left(n_{\mathrm{pr}}=1.5\right)$ this would mean that $C=0.64$.

2. So far, when defining the set of integration-points for emission $(D)$ and excitation $(E)$ to be considered in eq. 4.20 and eq. 4.21 , we ignored the effect of refraction that takes place at the boundary between air and prism. This effect, however, is quite significant and requires us to rethink the way we define $\mathrm{D}$ and E. One way to properly define these sets with the effect of refraction would be to follow each individual light ray (corresponding to a point $\Omega$ on the unit sphere) through the glass-air interface and deduce a new function $\Xi(\cdot)$ that would assign each $\Omega$ an image $\Omega_{\mathrm{r}}$. Once found, evaluation of $\Xi(\cdot)$ would allow us to define the set of integration points equally simple as already done above.

Alternatively, as an approximation, it is possible to consider a simplified view: instead of following each individual light ray in the field of view of our objective for each angle $\alpha$, we only consider refraction of the light on the beam axis of the objective (according to eq. 4.15) and account for the refraction of the other light rays by working with an effective numerical aperture $N A^{\text {eff: }}$

$$
N A^{\text {eff }}=\frac{N A}{n_{\text {glass }}} \quad \text { with } \quad \alpha^{\text {eff }}=\Omega-\arcsin \left(\frac{n_{\text {air }}}{n_{\mathrm{pr}}} \sin (\Omega-\alpha)\right)
$$

Although this approximation may seem crude it works extremely well for as long as the angle $\beta$ between beam axis and surface normal of the prism is not too big. This allows us now to write down eq. 4.19 more accurately:

$$
P_{\mathrm{Rad}}^{\rho}(\alpha)=\rho \cdot \frac{\mathrm{d} \sigma}{\mathrm{d} \Omega} \cdot K_{\#} \cdot C \cdot \underbrace{\int_{E\left(\alpha^{\mathrm{eff}}, N A_{\mathrm{Las}}^{\mathrm{eff}}\right)}\left(\frac{1}{\cos (\alpha)} M_{\#}^{\mathbf{P}_{\mathrm{L}}}\left(\Omega^{\prime}\right) \cdot \frac{\mathrm{d} P^{\mathrm{inc}}}{\mathrm{d} \Omega}\left(\Omega^{\prime}\right)\right) \mathrm{d} \Omega^{\prime}}_{\text {excitation }} \cdot \underbrace{\int_{D\left(\alpha^{\mathrm{eff}}, N A_{\mathrm{obj}}^{\mathrm{eff}}\right)} M_{\#}^{\mathbf{P}_{\mathrm{R}}}\left(\Omega^{\prime}\right) \mathrm{d} \Omega^{\prime}}_{\text {emission }}
$$




\subsubsection{No lens}

The setup with "no lens" in Fig. 42 would be the most direct simplification of the setup used in early studies [105-108]. A well collimated beam is required to maximize coupling into the very sharp (in angle) SPP resonance and, therefore, we initially use the laser beam as it is (with no focusing lens). Because of our choice of using the BS configuration, no lens can then be used for collection if we do not want to change the divergence of the exciting beam. The SPR signal (reflectivity) of the film can be simultaneously monitored by a simple photodiode detector which is attached to the rotating stage containing the prism and the film.

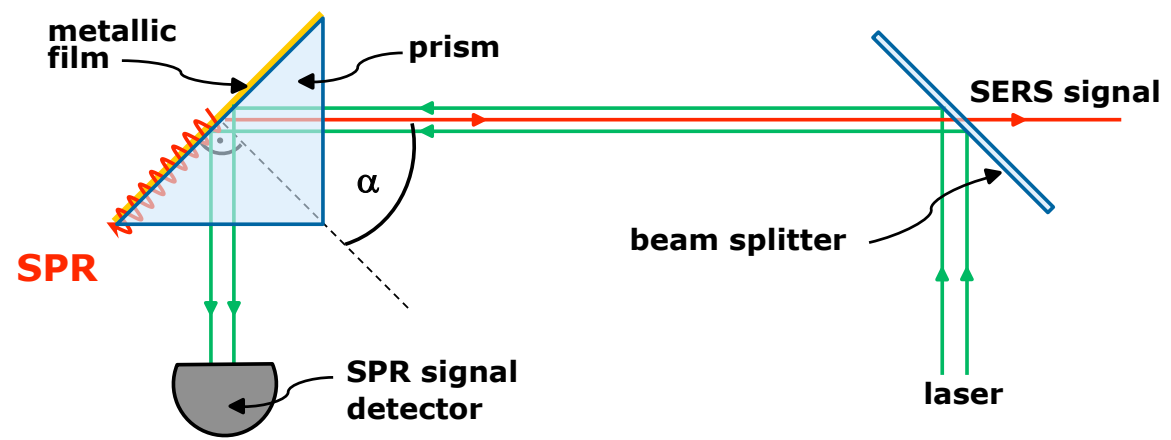

Fig. 42 :

Schematics of the optical setup for combined SPRS-SERS in the BS configuration with no focusing / collecting lens.

The reflectivity curve, for the situation where we have no lens, is shown in Fig. 44. Concurrently, Fig. 43a) shows the SERS signal $\left(590 \mathrm{~cm}^{-1}\right.$ Raman mode) of a monolayer of NB on Ag (50 nm film) in air at $647 \mathrm{~nm}$ excitation through a glass prism (index of refraction $n=1.5$ ) as a function of the internal incident angle $\alpha^{\text {eff }}$ (see Fig. 40). As expected, this signal only exists for TM polarization which can be seen in Fig. 43c).
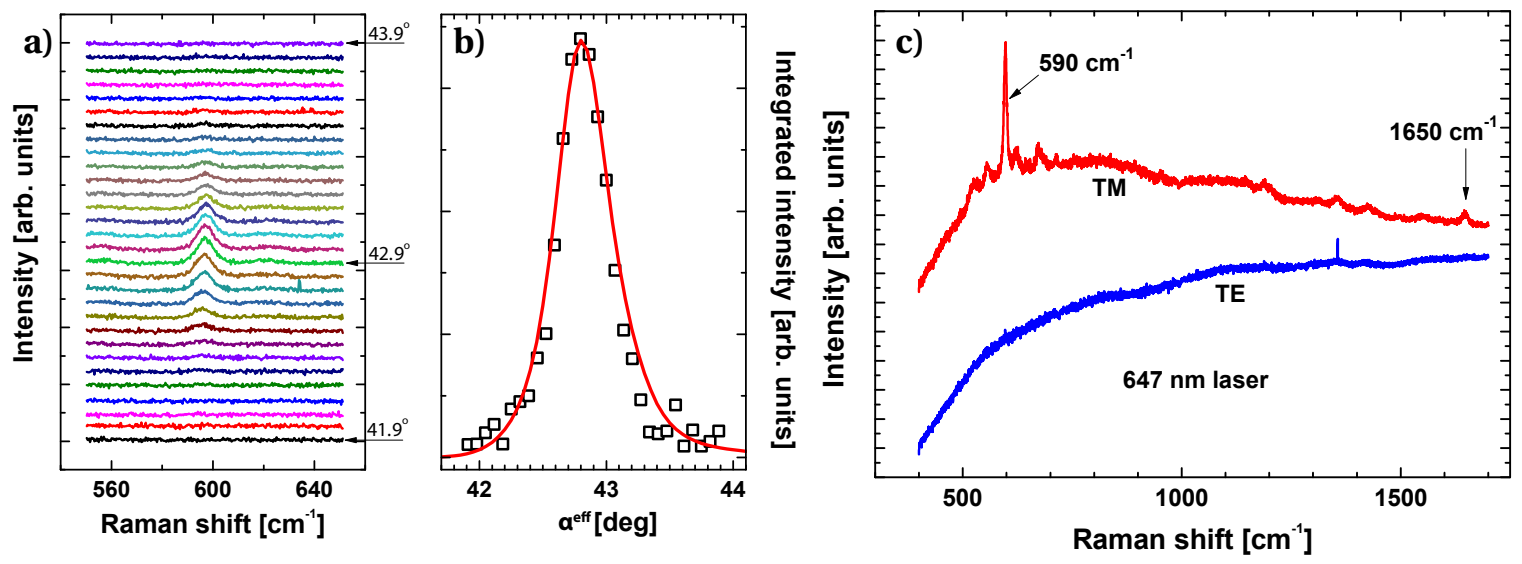

Fig. 43 : a) \& b) Experimental angle-dependent SERS signal (symbols) for the $590 \mathrm{~cm}^{-1}$ mode of NB deposited on an Ag/Air interface (50 $\mathrm{nm}$ Ag film) at $647 \mathrm{~nm}$ laser excitation (integration time $=18 \mathrm{sec}$, laser power $5 \mathrm{~mW}$ ). The theoretical prediction is shown as a solid line. The angle of incidence $\alpha^{\text {eff }}$ refers to internal angles inside the glass slide supporting the film (see Fig. 40). c) Full spectrum of NB at the optimum Kretschmann angle of the $590 \mathrm{~cm}^{-1}$ peak for TM and TE polarizations. The Raman peaks are observed only for TM polarization, as expected. The $1650 \mathrm{~cm}^{-1}$ mode of NB is visible, albeit with a smaller SERS enhancement factor, owing to the SPP resonance being relatively broad in wavelength. 
As can be appreciated from Fig. 43a), the SERS signal can be followed through the angular resonance. It goes from being invisible, when the laser is not properly coupled, to being an intense peak well above the noise level with a FWHM of $0.5^{\circ}$ (comparable to the the FWHM observed in reflectivity in Fig. 44 for the "no lens" case). The results in Fig. 43a) and b) are complemented by those in Fig. 43c), where the SERS signal in a much wider frequency range is shown for optimum excitation at the Kretschmann angle. This latter result demonstrates the point raised before: that the resonance is sharp in incident angle but broad in the spectral domain (allowing us to see simultaneously the 590 and $\sim 1640 \mathrm{~cm}^{-1}$ Raman modes of NB).

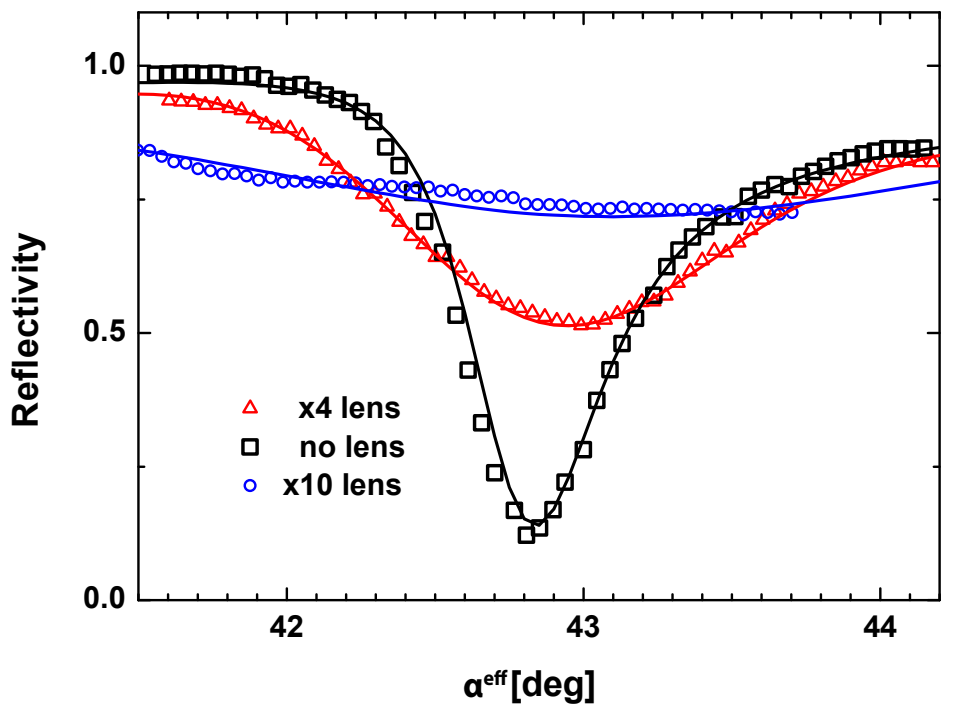

Fig. 44 :

Influence of a focusing lens on the angle-dependent reflectivity. Experimental data (symbols) clearly show the decreased coupling efficiency and broadening as the NA of the lens increases from the "no lens" case, to a $\times 4$ objective $\left(N A_{\times 4}=0.1\right)$ and to $a \times 10$ $\left(f=18 \mathrm{~mm}, N A_{\times 10}=0.25\right)$. The theoretical values (solid lines) are obtained by considering a collimated Gaussian beam of $0.72 \mathrm{~mm}$ waist radius incident on the lenses resulting in a spread of incident angles at the focal point.

It is quite remarkable that the Raman signal from a mono-layer of dye can be collected without a lens at all. Excluding any roughness contribution to SERS is however difficult with silver films because of their intrinsically low chemical stability. The agreement between theory and experiment in Fig. 43 and Fig. 44 indicates that roughness is not significant - it would otherwise be revealed in a broadening of the resonance. But this in itself is not a clear proof that roughness does not contribute to SERS. Only experiments on Au, where the absence of roughness can be assured more convincingly, will demonstrate unambiguously (as shown later) the ability to carry out SPR-SERS measurement on strictly flat surfaces. However, in order to be able to measure the much weaker SERS signals on Au films, it is first necessary to optimize our collection efficiency because with the current setup a large part of the Raman emission is being lost by the restriction that we are collecting only what comes strictly in the backscattering direction. This is a unique situation where the optical requirements to deliver the laser (i.e. a parallel beam) are incompatible with the optical requirements to collect most of the signal in the backscattering configuration. In the case of Fig. 42, the effective NA for the collection process (without a lens) is really small, and only defined by a narrow acceptance angle related to the paraxial tolerance around the exact optical axis of the spectrometer (we estimate the NA to be $\sim 0.02$ for our system). This was already partially explained (schematically) in Fig. 35. The only reason why a signal can be observed without a lens is because the whole Raman signal is "funneled" into the narrow Kretschmann cone, and we collect here a small fraction of it. We can view the film as behaving as a "1D lens"; a unique feature of a sharp angular resonance in emission. 


\subsubsection{Collection lens}

We could of course hope to detect a much larger signal by collecting a larger portion of the Kretschmann cone with a lens of larger NA. We can, for example, use the same setup as in Fig 42, but with a long working distance microscope objective (to allow for space for the prism). However, the improvement in collection efficiency has the price of losing the exciting beam collimation (which is now focused by the objective). This results in a decreased coupling into the SPP resonance and a broadening of the angle-dependent reflectivity, which effectively prevents any simultaneous SPR spectroscopy studies. This is illustrated in Fig. 45 for a $\times 10$ objective.

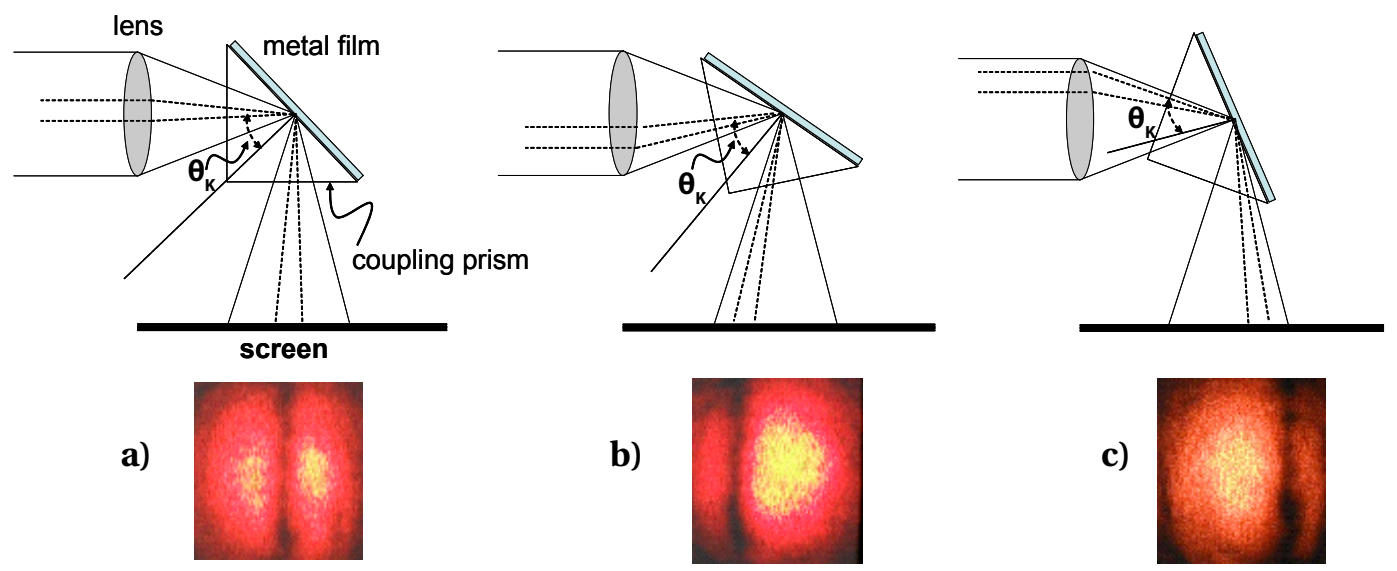

Fig. 45 Influence of a focusing lens on the angle-dependent reflectivity. This effect can be visualized with the $\times 10$ objective by projecting the image reflected from the sample onto a screen in the far-field, as shown in a)-c) for 3 angular positions. At resonance, a clear black fringe is visible (corresponding to full coupling into the SPP resonance) in the middle of the beam image. However, it is clear that the angular spread of the beam is much larger than that of the resonance resulting in a partial coupling only.

It is clear that in the converging beam produced with the $\times 10$ objective, only a fraction of the incident rays are at the Kretschmann-angle $\theta_{K}$ to couple to the SPP resonance. This is revealed in Figs. 45a-c) as a "dark fringe" in the far field of the reflected beam (which disappears - as expected when changing from TM to TE polarization). This dark fringe can easily be followed when rotating the sample and it gives a good idea of the "angular sharpness" of the resonance and of the angular spread of the exciting beam. If the angle of incidence is changed slightly (by a few degrees), the resonance fringe can be moved to one or the other side of the reflected spot in the far-field, as seen in Figs. 45a) and c). This is because different fractions of the angular spread of the incoming beam satisfy the resonance condition. This highlights one important aspect: simply using a lens for simultaneous excitation and collection will not be optimal, because only a small fraction of the incident power (only that at the right angle in the beam) couples into the resonance. This is shown more quantitatively in Fig. 44 through the reflectivity. It is clear that the use of lenses even with a small magnification-results in a strong decrease in coupling into the resonance and a concomitant larger broadening. This observation is predicted by theory when taking into account the finite width $(0.72 \mathrm{~mm}$ waist $)$ of the laser beam which is being focused by the lens and hence accounts for the broadening by a convolution of incoming angles. The natural (intrinsic) width of the resonance can therefore only be obtained with a collimated beam of small divergence. 


\subsubsection{Collection lens with BFP-lens}

The results of the previous sections highlights one of the basic conundrums of the use of the Kretschmann configuration for SERS. Unlike SPRS - that only monitors the reflectivity - we need to take into account in SERS both the delivery of the laser and the collection of the Raman emitted light. If we want to monitor the SERS signal in the BS configuration (a very convenient experimental choice) we would benefit from collecting at least part of the Kretschmann cone with a lens of high numerical aperture. However the presence of the lens is incompatible with a simultaneous measurement of SPR, for it prevents optimum coupling to deliver the laser. In the following, we will present a modified setup (see Fig. 46) that enables us to overcome this problem with minimal modifications to our standard Raman system.

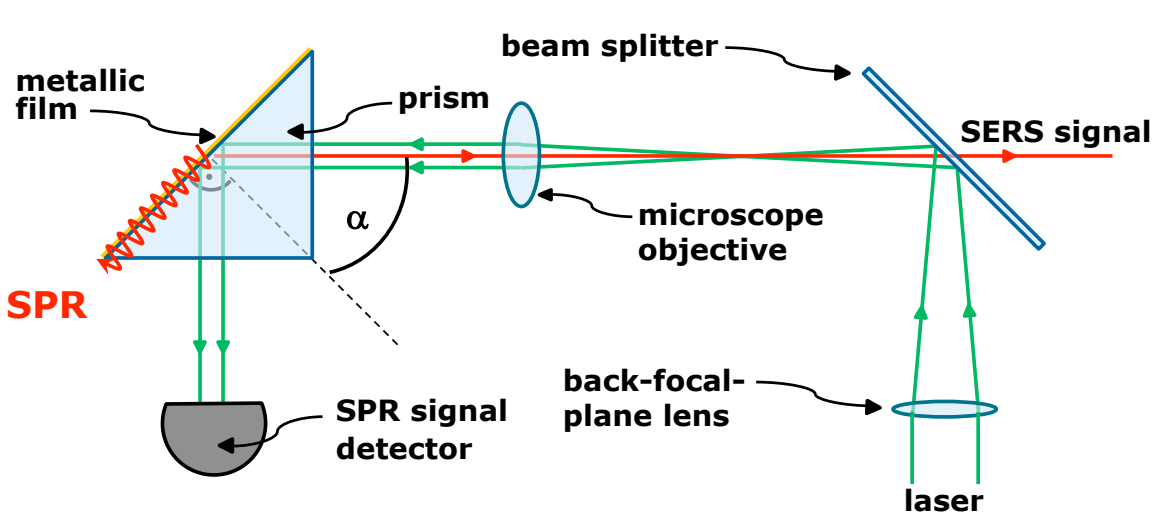

Fig. 46 :

Optical setup with BFP illumination. The BFP-lens focuses the light on the BFP of the microscope objective, thus achieving a parallel beam in excitation.

The principle is extremely simple: it consists of using an additional lens (typically with a long focal length) to focus the incident collimated laser beam on the BFP of the microscope objective. This allows us to create an almost parallel beam on the focal plane of the sample, and therefore combine the benefits of a high angular resolution in excitation (thanks to the collimated beam) with the much improved detection efficiency of a high- $N A$ objective. A variant of this approach, where the beam is focused off-centre in the BFP of a high-NA objective, is commonly used in Total Internal Reflection Fluorescence (TIRF), to excite beyond the critical angle [63].

The exact position of this additional lens (referred as the BFP-lens) along the optical axis is not critical and it can be adjusted experimentally by visually monitoring the beam divergence after the objective in the far-field. Care must however be taken to align the BFP-lens laterally so that the excitation follows the main optical path (and is therefore matched to the axis and the collection field-of-view of the objective).

As a proof of principle, we have applied this setup to the more difficult case of Au films, which are arguably much more relevant for applications such as SPRS. Their quality, stability, and absence of roughness can be assured with high reliability. However, the SERS signals on Au films in the KC is typically smaller than for Ag for two main reasons. Firstly, the SERS EF is predicted to be smaller at $647 \mathrm{~nm}$ due to the different dielectric functions of Au and Ag [2]. But perhaps more importantly, Ag films are much more likely to suffer from roughness, thereby enhancing dramatically the SERS signals. This is not desirable from the point of view of SPRS because roughness may affect the resonance quality (sharpness). It is not desirable either from the point of view of SERS, because it defeats the original purpose of uniform and controllable enhancements. 
Results obtained on a Au/air interface ( $50 \mathrm{~nm}$ Au film on glass) with the layout of Fig. 46 are displayed in Fig. 47 and complemented with further examples on the simultaneous performance of SERS and SPR signals for various collecting lenses in Fig. 48. We shall concentrate on the discussion of these figures for the rest of this section.
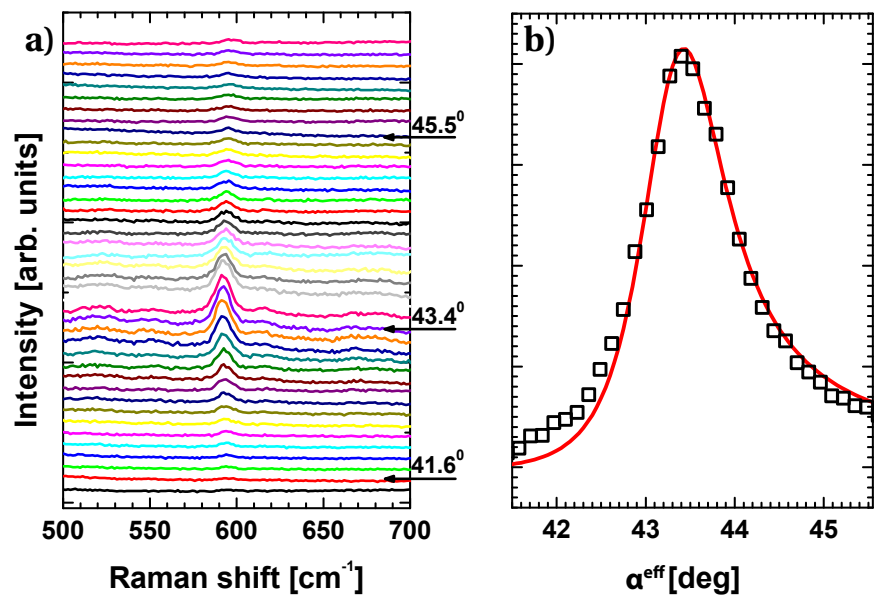

Fig. 47 :
Integrated intensity of the
$590 \mathrm{~cm}^{-1}$ mode of NB through the
resonance for a Au film (50 nm)
on glass and $647 \mathrm{~nm}$ laser excita-
tion (TM) using a $\times 10$ objective
and a BFP lens with $50 \mathrm{~cm}$ focal
length. The integration time was
$2 \times 5 \mathrm{sec}$, laser power $2.1 \mathrm{~mW}$.
The solid (red) line in b) is the
parameter-free theoretical predic-
tion for the SERS intensity. See the
text for further details.

Fig. 47b) shows the SERS intensity of the $590 \mathrm{~cm}^{-1}$ Raman mode of NB deposited on Au as a function of the incident (internal) angle $\alpha^{\text {eff }}$ for a $\times 10$ objective with a BFP-lens $(50 \mathrm{~cm}$ focal length). As before, the solid (red) line in Fig. 47 represents the parameter-free theoretical prediction which includes the effect of the numerical aperture of the collecting lens but with a collimated incident beam thanks to the BFP-lens. As can be appreciated, we can now obtain the SERS spectrum with the $\times 10$ objective, while we could hardly couple to the resonance at all in the situation without the BFP-lens (see Fig. 44).

The SERS data of the $\times 10$ objective with BFP-lens shown in Fig. $47 \mathrm{~b}$ ) is repeated in Fig. $48 \mathrm{a}$ ) to compare it with the other cases of a $\times 4$ objective with BFP illumination and $\mathrm{a} \times 4$ objective by itself. It is complemented in Fig. 48b) by the SPR spectra taken simultaneously for the three different conditions. Both the SERS signal and the SPR reflectivity data demonstrate how the coupling improves by including the BFP-lens:

- The signal increases for a higher numerical aperture objective $\left(N A_{\times 10}=0.25\right.$ as compared to $N A_{\times 4}=0.1$ ) benefiting in collection from a larger field of view, with the $\times 10$ objective giving the best signal.

- The coupling to the resonance improves to the point that the $\times 4$ and $\times 10$ objectives achieve almost zero reflectivity at the resonance (meaning that most of the power of the laser is being funneled into SPPs on the film).

It is worth pointing out that this situation is very different from Fig. 44 where the coupling was heavily affected by the beam-divergence introduced by the microscope objective. The other important point to emphasize here is that all the lines in Fig. 48a) and b) are parameter-free predictions from theory, except for a trivial scaling factor for the integrated SERS intensity (since the Raman intensity, as given by the CCD counts, depends on many factors that are purely technical, the scaling factor has no physical meaning). We judge the agreement to be outstanding, thus 
demonstrating the potential predicting power of the technique.This is a point that cannot be underestimated in a technique like SERS, where exact quantification and modeling are in many cases somewhat disconnected from experimental evidence, or only connected at a qualitative level. We believe the data in Fig. 47 and Fig. 48 and the quality of the agreement with theory proves beyond doubt the potential of combined SPR-SERS spectroscopy.
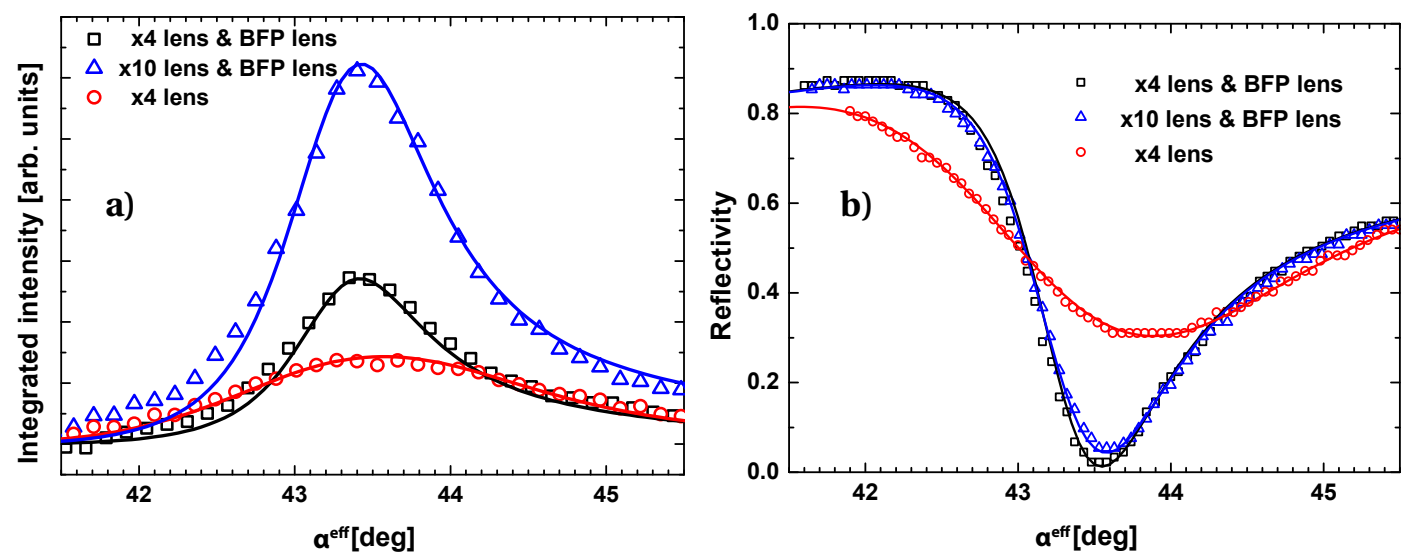

Fig. 48 : a) Integrated Raman intensity of the $590 \mathrm{~cm}^{-1}$ mode of NB and b) SPR (reflectivity) signal for various backscattering Kretschmann configurations at a Aulair interface. Solid lines are theoretical predictions that follow from angular averaging. The integration time for the Raman spectra was $2 \times 5 \mathrm{sec}$, laser power $6.4 \mathrm{~mW}(\times 4$ with BFP), $2.1 \mathrm{~mW}(\times 10$ with $B F P)$ and $2.3 \mathrm{~nm}(\times 4)$.

In order to fully realize the potential of combining the commercially available SPR systems with SERS, in a dual-detection capability, it is also necessary to demonstrate its viability at the commonly used Au/water interface (e.g. for doing SPR in biology [64-68]). This is explicitly shown in Fig. 49. For these experiments we used a sapphire prism, which has a larger index of refraction ( $n=1.77)$ and therefore is more suited to achieve the resonance condition with water $(n=1.33)$ at a reasonable incident angle. A liquid cell was specially designed to attach to the metal film glass slide. Fig. 49a) shows both the actual raw data for the 590 and $1650 \mathrm{~cm}^{-1}$ peaks of NB through the resonance, and the integrated intensity of the $590 \mathrm{~cm}^{-1}$ mode as a function of the internal (in glass slide) incident angle $\alpha^{\text {eff }}$. Fig. 49b) complements the picture with the simultaneous measurement of the SPR signal. The experiment was carried out with a $\times 4$ objective and shows an almost perfect coupling to the resonance in the dip of the reflectivity. As before, the solid (red) lines in Fig. 49a) and Fig. 49b) are parameter-free theoretical predictions. There is a whole set of possibilities that are opened up by this result. The connection with biological applications of SPR-spectroscopy already pointed out is an obvious one. But it might also prove to be a useful tool in the study of other phenomena like surface-enhanced fluorescence [41]. If the analyte is dissolved in the liquid, the evanescent field of the SPPs penetrates a considerable distance into it. Molecules that are not directly attached to the surface do not have their fluorescence completely quenched (see Chapter 1). 

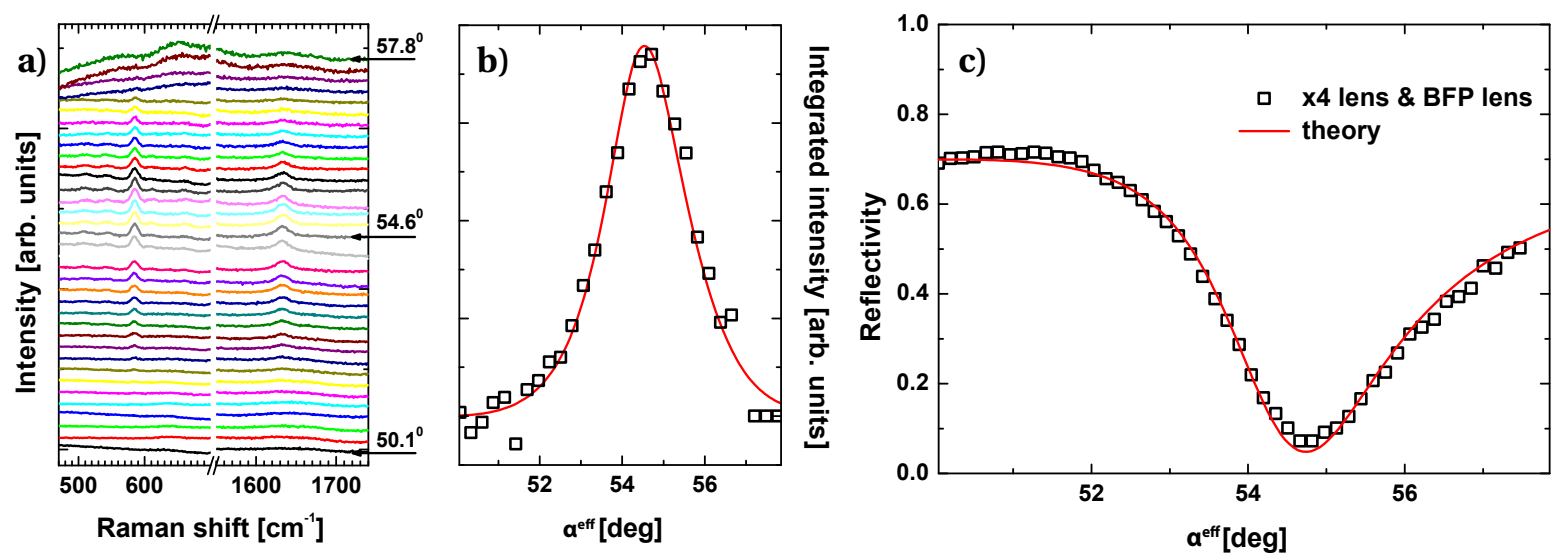

Fig. 49 : a) Raw data for the $590 \mathrm{~cm}^{-1}$ and $1640 \mathrm{~cm}^{-1}$ Raman modes of NB and integrated intensity of the $590 \mathrm{~cm}^{-1}$ mode across the resonance. Integration time was $2 \times 2 \mathrm{sec}$, laser power $2.0 \mathrm{~mW}$. These experiments were carried out on a $50 \mathrm{~nm}$ Au film in water at $633 \mathrm{~nm}$, excitation with $\times 10$ BFP illumination and TM polarization. b) Integrated Raman intensity of the data shown in a). c) Simultaneous measurement of the reflectivity (SPR signal) showing a high degree of coupling. The solid (red) curves in b) and c) are the theoretical predictions. For this experiment, the dye is deposited on the Au film beforehand, and then exposed to a liquid cell with water; i.e. the dye is not dissolved in solution. See the text for further details. 


\subsection{Collecting the Kretschmann cone - the large NA setup}

In the previous section the prism setup was discussed in extensive detail, showing that concomitant SERS and SPRS is possible on a monolayer of NB. This was done on Au as well as Ag - in air as well as water. Even though the setup's performance was improved significantly by introducing a back-focal-plane lens, the fact remains that most of the enhanced signal on the glass side (i.e. most of the Kretschmann cone) is wasted. This is the motivation to discuss in this section the "large NA setup" - a configuration shown in Fig. 37d) that allows not only to collect the whole Kretschmann cone, but also introduces the capability to do microscopy. This latter aspect urges us to analyze the technical aspects of this implementation in more detail, with a particular emphasis on the factors determining spot-size, coupling efficiency to SPP, and spatial resolution.

\subsubsection{Experimental setup}

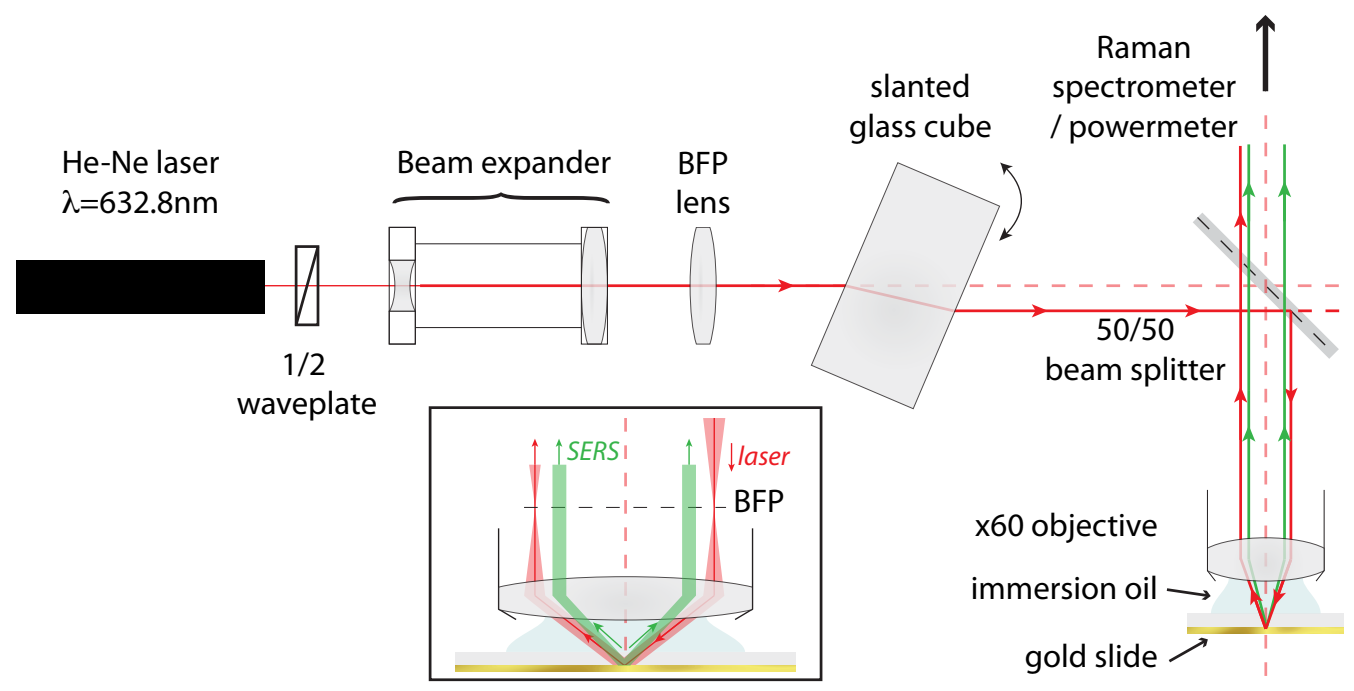

Fig. 50 : Schematic representation of the experimental setup. A He-Ne laser beam $(\lambda=632.8 \mathrm{~nm})$ is directed towards the sample through the oil-immersion TIRF objective of a microscope. The direction of the linear polarization of the laser beam is adjusted with a half-wave plate. The beam is then expanded, and directed toward a convergent lens with focal distance $f=400 \mathrm{~mm}$ that is used to focus the beam onto the BFP of the microscope objective, thereby producing a parallel beam when it reaches the sample. A glass cube is placed onto a motorized rotating stage, which enables a parallel displacement of the beam when it enters the microscope. As a result, the ray exits the objective at an angle directly related to the cube's rotation. The SERS signal, and the reflectivity (assessing the coupling to SPPs) are both monitored in backscattering through the beam-splitter, as indicated in the figure. For clarity and display purposes only the laser light is shown in red, and the Raman light (to be collected) in green. Note that the minute displacement between the Raman and laser light (produced by the dispersion of the resonance) has been exaggerated. Inset: detailed view of the light beam near the microscope objective. Beam converges at the back-focal plane, and emerges collimated at the sample.

Fig. 50 presents in more details the experimental setup of Fig. 37d) that was developed to combine 
most efficiently standard microscopic SERS measurements with a Kretschmann excitation scheme in the backscattering configuration. Using a high-NA objective, the full Kretschmann cone of emitted radiation can be collected for all incident angles. This is, by itself, an important improvement of this setup compared to conventional backscattering prism-based coupling versions of the KC for SERS [60]. Also, as a result the enhancement factor due to the reemission (Raman) process was constant in our experiments and the angular dependence is therefore dictated by the local field enhancement (for excitation) only, i.e. $\propto\left|E_{\mathrm{Loc}}\right|^{2} /\left|E_{0}\right|^{2}$. Spatial resolution was achieved through the use of a standard optical microscope mounted with a high-NA objective (Olympus, Apo N, $\times 60$, $N A=1.49$, oil immersion) with standard specifications for TIRF microscopy. Coupling of the incident laser to the surface mode was achieved by shifting the beam using a slanted glass cube (see Fig. 50), whereby the beam axis emerges from the objective at a specific angle $\alpha^{\text {eff }}$ with respect to the Au surface normal. Section 4.6.3 provides further experimental and theoretical details on the connection between the angle $\alpha$ (between beam axis and the cube's surface normal) and the angle of incidence on the sample $\alpha^{\text {eff }}$. In order to optimize the coupling to the resonance, a collimated beam is required. To this end, a BFP-lens (focal length $400 \mathrm{~mm}$ ) was placed before the entrance of the microscope [62]. The exact position of this lens is crucial and was finely adjusted by observing the condition of minimum divergence of the beam (being transmitted through a transparent substrate like glass) in the far-field after the objective.

In order to understand how the experimental setup behaves in controlled situations, we have performed basic reflectivity experiments on glass and Au in TM-polarization and found good agreement with the corresponding theoretical expectations.

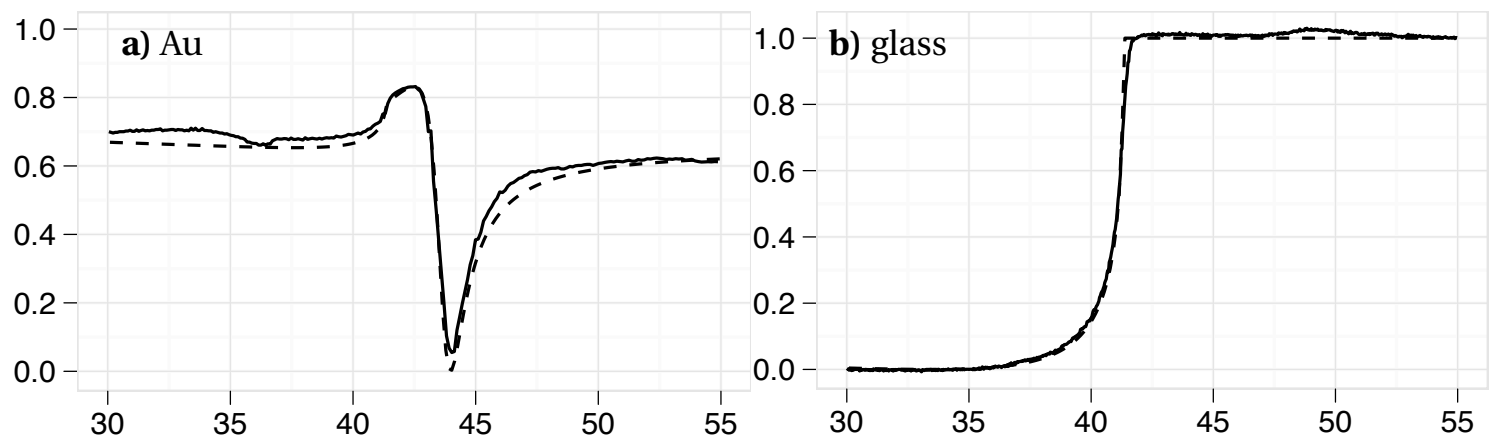

Fig. 51 : Experimental (solid lines) and theoretical (dashed lines) TM-polarized reflectivities on a) Au and b) glass as a function of the incident (internal) angle $\alpha^{\mathrm{eff}}$ (see Fig. 52). The experiment is done for an expanded beam (i.e. for a beam with a minimum divergence). The agreement between theory and experiment is very good and reveals that there is no spurious experimental distortion in the beam path.

Fig. 51 shows the reflectivity data as a function of angle of incidence $\alpha^{\text {eff }}$ for Au and glass, respectively. The specular reflectivity was measured in backscattering, as in Fig. 50. The figure also shows the theoretical expectation for the reflectivity in the different cases (dashed lines). As can be appreciated, the agreement between theory and experiment is quite satisfactory. Crucially, the region of total internal reflection in b) for the glass/air interface $\geq 42^{\circ}$ presents a uniform reflectivity of unity, spanning the full range of the plasmon resonance. Immediately before the critical edge of total internal reflection, the Brewster angle for the glass/air interface is marked with minimum reflectivity close to 0 , confirming the well-maintained TM-polarisation of the incident beam. Im- 
perfection with respect to theory originate from small spurious reflections in the optical elements. Wider angular scans (not shown) were also performed to verify that the reflectivity was symmetric for positive and negative values of $\alpha^{\text {eff }}$, i.e. with the beam shifted to one side or the other from the axis. Furthermore the good collimation of the beam was assessed by the angular scan of the reflectivity in the Kretschmann configuration (TM-polarization). With Au substrates, better than 95\% coupling was achieved, measured from the highest-to-lowest reflectivity values, and a resonance width comparable with the theoretical prediction.

\subsubsection{Sample preparation}

NB molecules were deposited onto the metal surface as described in chapter 3: a Au film $(45 \mathrm{~nm}$ thickness evaporated on glass with an intermediate $2 \mathrm{~nm}$ titanium adhesion layer) was immersed in a $1 \mu \mathrm{M}$ NB solution in water for $\sim 30 \mathrm{~min}$ and then thoroughly washed with ultra-pure water and dried. Films prepared with this method achieve a monolayer deposition on the surface (corroborated by independent electrochemical measurements) and present a good overall uniformity of the SERS signal at different positions.

All Raman spectra were measured using the BS configuration of Fig. 50 with a Jobin-Yvon T64000 spectrometer described in Chapter 1. Sometimes, to improve the collection efficiency, the double pre-monochromator stage was replaced with a notch filter for $633 \mathrm{~nm}$ laser excitation. A polarizer was permanently used for detection.

\subsubsection{Angle of incidence as a function of cube rotation}

The essential geometrical aspects of the problem that link the rotation of the cube with respect to the beam axis $\alpha$ with the angle of incidence $\alpha^{\text {eff }}$ are displayed in Fig. 52a). The mathematical connection between $\alpha^{\text {eff }}$ and $\alpha$ is established through eq. 4.27 and eq. 4.28. For a start,

$$
s=e \cdot \frac{\sin \left(\alpha-\alpha^{\prime}\right)}{\cos \alpha^{\prime}} \quad \text { with } \quad \alpha^{\prime}=\arcsin \left(\sin \alpha / n_{\text {cube }}\right)
$$

describes the parallel shift of the beam through a parallelepiped of refractive index $n_{\text {cube }}=1.5$ and thickness $e=25.4 \mathrm{~mm}$. Abbe's sine condition provides the link between the off-axis beam and the incident angle:

$$
\alpha^{\mathrm{eff}}=\arcsin (s / f)
$$

For our system, $f=4.2 \mathrm{~mm}$ is the effective focal length of the objective.

In Fig. 52c) we show a plot of $\alpha$ as a function of $\alpha^{\text {eff }}$. As can be observed, the connection between the two is fairly linear up to $\alpha^{\text {eff }} \sim 50^{\circ}$, where nonlinearity rapidly sets in. The Kretschmann resonance is observed around $\alpha^{\text {eff }} \sim 44^{\circ}$ for $45 \mathrm{~nm}$ films of either Ag and Au in air (the resonance angle is fairly insensitive to thickness and wavelength [2]). The incidence angle can also be measured experimentally using a graduated scale on a dielectric cube, as depicted in the photo of Fig. 52b). The experimental determination of the calibration of the angle of incidence is presented in Fig. 52c) against the theoretical expectation. Excellent agreement between theory and experiment is observed, for an effective focal length $f=4.2 \mathrm{~mm}$. Once determined, this value was fixed 
in the angular conversions. A slight offset of the origin was allowed to vary as a free parameter in order to match the observed plasmon resonance position with the theoretical predictions.

a)

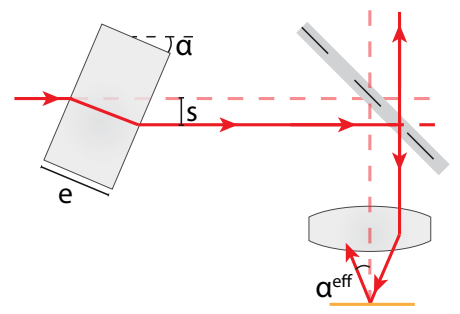

b)

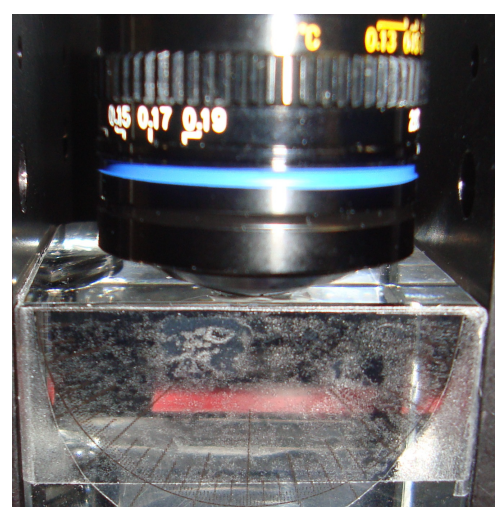

c)

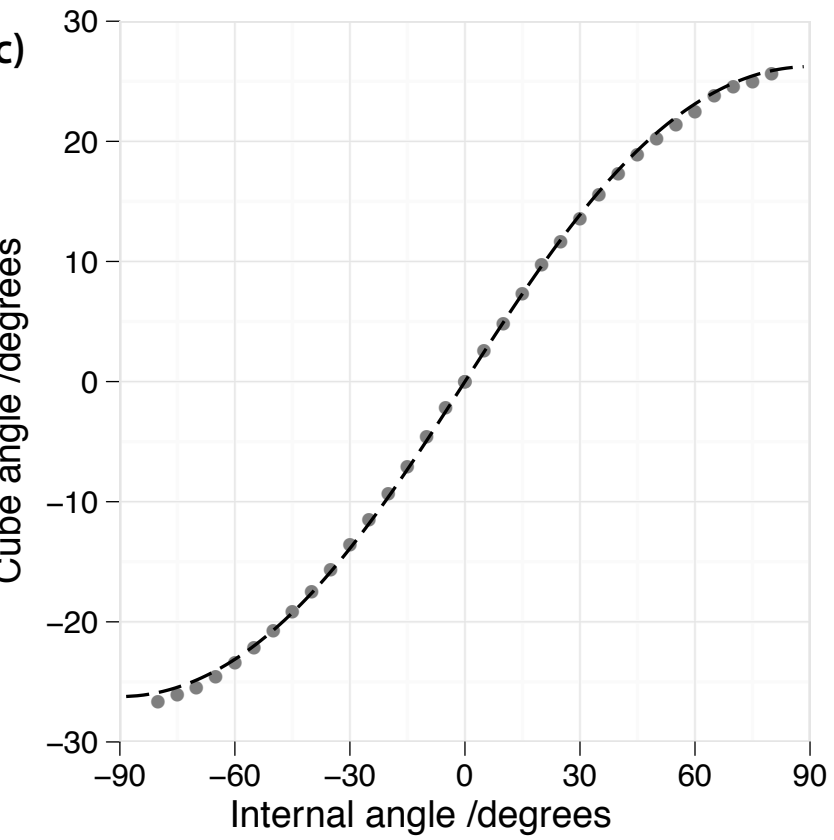

Fig. 52 : a) Diagrammatic representation of the beam displacement used for the angle scan. A laser beam traverses a glass cube (refractive index 1.5) rotated by an angle $\alpha$ and undergoes a parallel shift s. After being redirected by a beam-splitter, the shifted beam enters the microscope objective off-axis, and reaches the sample with an angle $\alpha^{\mathrm{eff}}$. b) Photograph of the experimental apparatus used to measure the variation of incident angle vs cube angle. A protractor printed on a transparency was glued onto a glass cube of refractive index 1.5 matching that of the immersion oil of the TIRF objective. By monitoring the position of the beam intersecting the angular marks along the edge of the cube, a reading of the incident angle was correlated with the computer-controlled rotation stage of the glass cube shifting the beam. c) Measured (points) and predicted (dashed line) variation of the incident angle against the cube's angle. Negative angles refer to the beam incident from the other side of the normal.

\subsubsection{Trade-off between resolution and coupling to SPP}

The setup presented in Fig. 50 uses a back-focal plane lens to realize a collimated beam incident on the sample, a crucial condition to achieve optimum coupling to SPPs, together with high LFEF. We now examine in detail the convoluted effects of beam divergence and spot size on the resonant coupling to SPP and the spatial resolution. This is clearly a crucial aspect for both SPR microscopy and SERS in the Kretschmann configuration, but the following discussion is in fact more general, and would indeed apply to any SPR experiments.

An ideal plane wave impinging on a thin metal film in the Kretschmann configuration can undergo total absorption at the exact angle of incidence corresponding to the SPP resonance. A real laser 
beam, however, has an intrinsic spread of angles that varies with the beam size: the smaller the size of the beam, the greater its angular spread thereby impairing ideal coupling ${ }^{3}$. On the other hand the beam size also dictates power density (a crucial parameter in many applications) and spatial resolution ${ }^{4}$ : the smaller the illuminated spot the better the resolution. From that point of

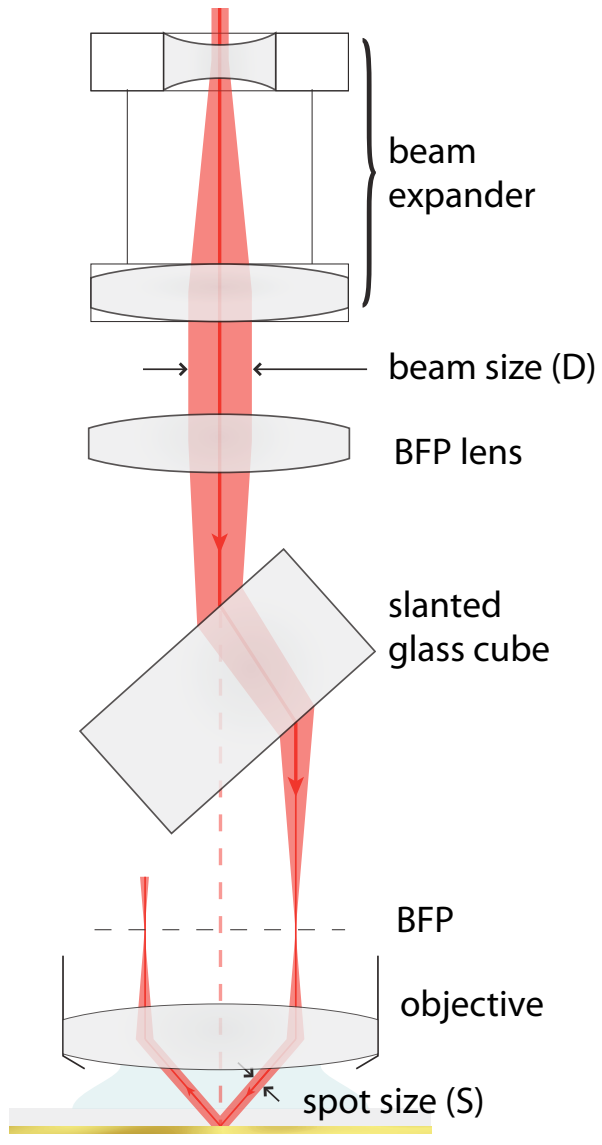

Fig. 53 : Simplified optical layout illustrating the ray-optics approximation of the beam (not to scale for clarity). The spot size $(S)$ is directly proportional to the diameter $(D)$ of the off-axis beam feeding the Back Focal Plane (BFP) lens after the expander. view a small spot size is clearly desirable. Inherent in the spot-size, there is therefore an unavoidable trade-off between spatial resolution and the coupling efficiency which needs to be well understood in order to decide on the best strategy for a given desired spatial resolution in SPR/SERS microscopy. As we now show, our proposed set-up is very flexible in this regard thanks to the beam expander used in excitation.

Figure 53 shows a simplified schematic of the excitation path of the beam, where we have included only the essentials. The beam increases to a diameter $D$ after the expander, impinges onto the BFP lens, and is shifted offcenter by the slanted glass cube. The lens focuses the beam onto the back focal plane of the objective, whereupon it emerges as a parallel beam with an angle (e.g. the resonance Kretschmann angle) onto the sample with a cross-sectional area $S$ which is equivalent to the spot size at normal incidence. As is evident from Fig. 53, in the ray optics description of the excitation path (which applies for the relatively large spot sizes considered here), the spot size $S$ is directly proportional to the beam size $D$ after the expander. Hence, a reduction in the excitation beam diameter (obtained by changing or removing the expander) yields a concomitant reduction in spot size until diffraction effects become important at small sizes (of the order of a micrometer-sized spot).

\footnotetext{
${ }^{3}$ If the ray optics picture was valid all the way to very small beam sizes $(\sim \lambda)$, we could potentially create a very small spot size $S$ by reducing $D$ as much as possible, and still achieve a very good coupling to SPPs with a parallel beam. This does not happen in reality because the product of the beam size with the beam divergence (étendue) cannot be made smaller than the original value characterizing the laser beam. Accordingly, a small spot is always associated with some degree of spread in the incidence angles on the sample and, therefore, a worsening of the coupling conditions to SPPs.

${ }^{4}$ It should be noted that spatial filtering can also be carried out in the collection part of the system, by limiting the area of the sample image that is spectrally analyzed using slits or pinholes. The spatial resolution is then dictated by the resolving power of the microscope objective; in our case (high NA objective), it is therefore in principle diffractionlimited. This approach is however not very efficient, in particular if the spot size is much larger than the desired spatial resolution.
} 
Within the ray optics approximation, the rays reaching the sample should be parallel and inclined at the Kretschmann excitation angle (optimum coupling to SPPs), but as mentioned already, this is no longer the case for small spot sizes. A combination of perfectly collinear plane waves cannot describe a beam with finite spatial extent; instead, one must consider a continuous distribution of plane waves with slightly diverging wave-vectors. In the approximation of large enough beam diameter, this corresponds to a bundle of light rays with a slight spread of angles around the mean beam direction (given by the Kretschmann angle). In the next paragraphs, we use a simple model

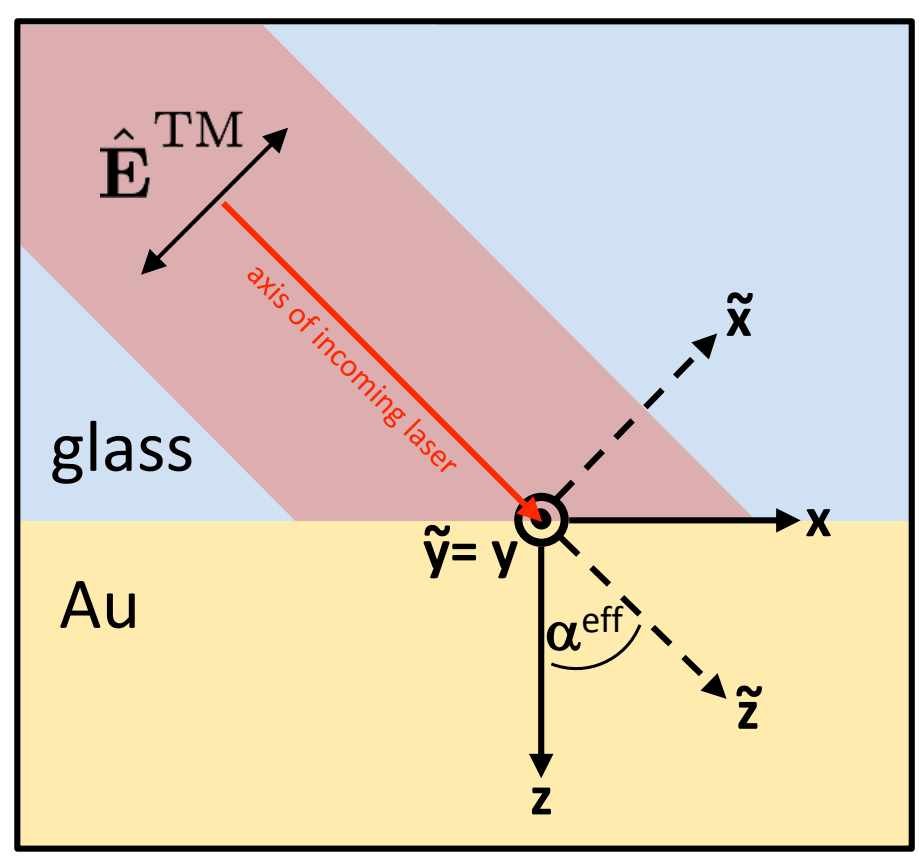

Fig. 54 : Gaussian beam impinging Au surface at an angle $\alpha$. to assess the trade-off between spatial resolution and coupling, and we compare it with direct experimental results in our system. We can use a simplified theory to account for the beam-like aspects of the excitation that still uses the ray-optics description but explicitly allows for a spread of incident angles. When the mean angle is at resonance, part of the beam is slightly off-resonance, thus leading to a residual reflectivity and a broadening of the reflectivity dispersion curve. To evaluate the detrimental effect of reducing the beam size on its coupling to SPPs, we follow here the approach proposed by Villatoro and coworkers [119].

The incident laser $(\lambda=633 \mathrm{~nm})$ is modeled as a Gaussian beam of waist radius $\omega_{0}$ (corresponding to the half-width at $1 / e^{2}$ ). After traversing off-axis the objective it illuminates a $50 \mathrm{~nm}$ Au film (with $2 \mathrm{~nm}$ Ti adhesion layer) from the substrate side (refractive index of glass is 1.53) at an angle $\alpha^{\text {eff }}$. This situation is schematically depicted in Fig. 54. The electric field $\mathbf{E}_{\text {inc }}$ and intensity $I_{\text {inc }}=\left|\mathbf{E}_{\text {inc }}\right|^{2}$ at $\tilde{z} \equiv 0$ can then be written as follows:

$$
\mathbf{E}_{\text {inc }}(\tilde{x}, \tilde{y})=\mathbf{E}_{0} \cdot \exp \left(-\frac{\tilde{x}^{2}+\tilde{y}^{2}}{\omega_{0}^{2}}\right) \text { and } I_{\text {inc }}=I_{0} \cdot \exp \left(-2 \frac{\tilde{x}^{2}+\tilde{y}^{2}}{\omega_{0}^{2}}\right)
$$

Since the numerical calculations done with the SPlaC code for planar surfaces are carried out a) on plane-waves of infinite size and b) in the reference frame of the Au surface, we need to first find those plane-wave contributions for our Gaussian beam by Fourier transforming it into k-space and then transform those contributions from one to the other reference frame. Let us start by Fourier transforming the intensity. This renders:

$$
\begin{aligned}
\hat{I}_{\text {inc }}\left(\tilde{k}_{x}, \tilde{k}_{y}\right) & :=\mathscr{F}\left(I_{\text {inc }}\right)=\left(\frac{1}{\sqrt{2 \pi}}\right)^{2} \int_{\mathbb{R}} \int_{\mathbb{R}} I_{\text {inc }}(\tilde{x}, \tilde{y}) \exp \left[-i\left(\tilde{k}_{x} \cdot \tilde{x}+\tilde{k}_{y} \cdot \tilde{y}\right)\right] \mathrm{d} \tilde{x} \mathrm{~d} \tilde{y} \\
& =\omega_{0}^{2} \cdot I_{0} \cdot \exp \left[-\frac{\omega_{0}^{2}}{2}\left(\tilde{k}_{x}^{2}+\tilde{k}_{y}^{2}\right)\right]
\end{aligned}
$$


In order to perform the variable transformation $\left(\tilde{k}_{x}, \tilde{k}_{y}\right) \longrightarrow\left(k_{x}, k_{y}\right)$ the following relations (obvious from Fig. 54) are helpful:

$$
\begin{aligned}
& k_{x}=\tilde{k}_{z} \sin \left(\alpha^{\mathrm{eff}}\right)+\tilde{k}_{x} \cos \left(\alpha^{\mathrm{eff}}\right) \\
& k_{y}=\tilde{k}_{y}
\end{aligned}
$$

Since $\tilde{k}_{z} \approx n \cdot k_{0}$ (assuming $\left|k_{x}\right|,\left|k_{y}\right| \ll\left|k_{z}\right|$ and with $\left.k_{0}=2 \pi / \lambda\right)$ it is easy to write now $\mathscr{F}\left(I_{\text {inc }}\right)$ as a function of $k_{x}$ and $k_{y}$ :

$$
\hat{I}_{\text {inc }}\left(k_{x}, k_{y}\right)=\omega_{0}^{2} \cdot I_{0} \cdot \underbrace{\exp \left[-\frac{\omega_{0}^{2}}{2} \cdot\left(\frac{k_{x}-n k_{0} \sin \left(\alpha^{\mathrm{eff}}\right)}{\cos \left(\alpha^{\mathrm{eff}}\right)}\right)^{2}\right]}_{p\left(k_{x}\right)} \cdot \exp \left[-\frac{\omega_{0}^{2}}{2} k_{y}^{2}\right]
$$

In this expression the function $p\left(k_{x}, \alpha^{\text {eff }}\right)$ is of central interest to us. When divided by its integration value

$$
C_{\alpha^{\mathrm{eff}}}:=\int_{\mathbb{R}} p\left(k_{x}, \alpha^{\mathrm{eff}}\right) \mathrm{d} k_{x}=\frac{\omega_{0}}{\sqrt{2 \pi} \cos \left(\alpha^{\mathrm{eff}}\right)}
$$

it describes the normalized probability density $p_{\text {norm }}\left(k_{x}, \alpha^{\mathrm{eff}}\right)$ for the distribution of $k_{x}$ values in the incoming beam as a function of the angle $\alpha^{\text {eff }}$. This allows us to calculate the effective response for the reflectivity $R\left(\alpha^{\text {eff }}\right)$ and the LFEF $F\left(\alpha^{\text {eff }}\right)$ as a simple convolution:

$$
\begin{aligned}
& R\left(\alpha^{\text {eff }}\right)=\int_{\mathbb{R}} p_{\text {norm }}\left(k_{x}, \alpha^{\text {eff }}\right) \cdot R\left(k_{x}\right) \mathrm{d} k_{x} \\
& F\left(\alpha^{\text {eff }}\right)=\int_{\mathbb{R}} p_{\text {norm }}\left(k_{x}, \alpha^{\text {eff }}\right) \cdot F\left(k_{x}\right) \mathrm{d} k_{x}
\end{aligned}
$$

The values for $F\left(k_{x}\right)$ and $R\left(k_{x}\right)$ are easily obtained with the SPLaC program. Since the latter works with the angle of incidence $\theta$ of an infinite plane wave (rather than $k_{x}$ ) one simply has to remember that the corresponding $k_{x}=n k_{0} \cdot \sin (\theta)$ and make the appropriate transformation:

$$
F\left(k_{x}\right)=F_{\mathrm{SPlaC}}(\underbrace{\arcsin \left(k_{x} / n k_{0}\right)}_{\theta})
$$

A similar step is required for the reflectivity $R$. The results of this averaging are shown in Fig. 55 for varying beam waist radii, and show a clear broadening of the sharp spectral features associated with the plasmon resonance.

Experimentally, the beam size was varied by removing the beam-expander in Fig. 50. The beam size reaching the sample was measured at normal incidence, using a knife-edge technique (see the supplementary information of Ref. [24]) to extract the beam waist radius $w_{0}$.

This comparison is presented in Fig. 55a) and b), with two different spot sizes (radius of the spots: $\sim 3 \mu \mathrm{m}$ and $\sim 15 \mu \mathrm{m}$ ). As can be appreciated from the figure, the smaller the spot size, the lower the efficiency of the SERS signal, when properly normalized by the incident power (in $\frac{\text { counts }}{\mathrm{sec} \cdot \mathrm{mW}}$ ). This is a direct consequence of the fact that, despite having the same collection efficiency in both cases, the coupling to SPPs is compromised when the spot size is reduced as described in the previous paragraph. This is also revealed in the broadening of the resonance, which is narrower (better coupling) for the larger spot (less spread in incident angles). 
The previous studies demonstrate that there is an unavoidable trade-off between spot-size and beam divergence in SPR/SERS microscopy in general and in our proposed set-up in particular. If the beam divergence is reduced to maximize coupling to SPP, then the minimum spot-size is limited to about $\sim 15-20 \mu \mathrm{m}$ with Au substrates. The situation is considerably worse for $\mathrm{Ag}(\sim 300 \mu \mathrm{m}$ for optimum coupling) owing to its narrower SPR response [119]). If the spot-size is reduced to about $\sim 5 \mu \mathrm{m}$ diameter, then the best achievable coupling to SPP is of the order of $30-35 \%$ only. Which combination of spot-size/beam divergence is chosen therefore depends on the application. For example, if a spatial resolution of $\sim 15-20 \mu \mathrm{m}$ is sufficient, and the input power density not critical, then one should use these relatively large spot sizes where the best coupling to SPP is obtained. In this latter case, the true angle-dependent SPR reflectivity can then be measured together with a large SERS intensity (since both excitation and emission are efficient here).

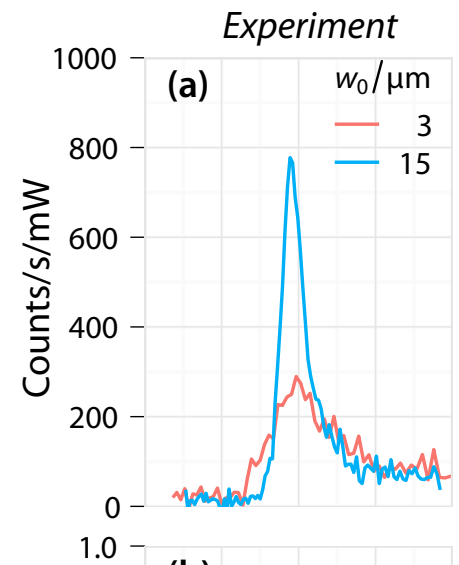

(b)

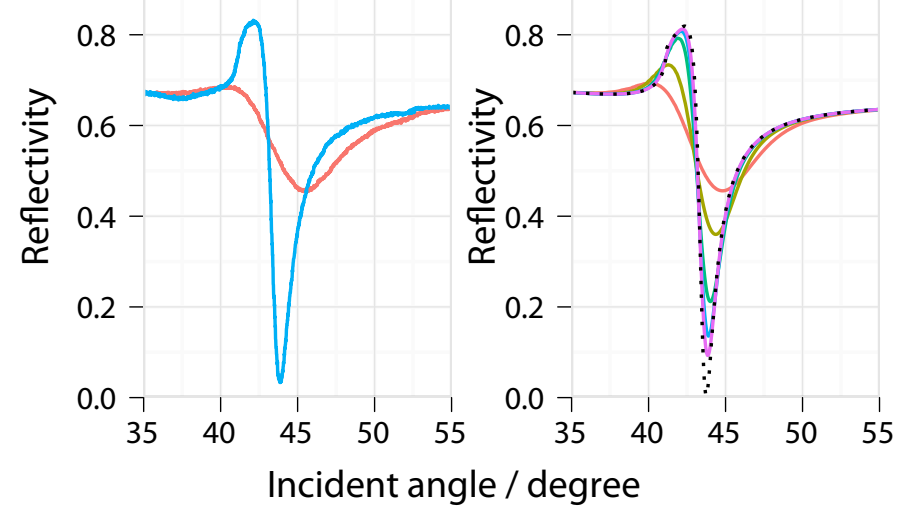

Fig. 55 :

Influence of the beam size on the coupling efficiency. a) Integrated Raman counts normalized by the integration time and incident laser power. b) Experimental reflectivity curves for the same configuration, normalized by the maximum value of the corresponding theoretical predictions. The beam waist radii corresponding to the two different experimental configurations were measured as $15 \mu \mathrm{m}$ (beam expander), and $3 \mu \mathrm{m}$ (no beam expander). c) Theoretical LFEF for a dipole parallel to the interface; d) reflectivity curves for the same configuration. In dashed lines is shown the solution for plane wave illumination (which achieves the best coupling condition).

Three alternative and arguably complementary strategies are possible to improve the spatial resolution:

- The imaging approach, which is based on projecting the sample image onto a CCD array, and is the basis for SPR microscopy. The main disadvantage here is that obtaining the SERS spectrum is difficult, although fluorescence intensity images (and possibly integrated SERS intensities within a given spectral window) can in principle be obtained using spectral filters.

- Spatial filtering in collection, where only a small area of the image (defined by the use of pinholes) is spectrally analyzed. Both SPR reflectivity and SERS spectra can then be measured, but the efficiency of the process is not very good as the spot-size is larger, or much 
larger than the analyzed area. This could however be compensated by increased laser power, at the expense of over-heating.

- Spot size reduction: in this case, the power density illuminating the area of interest is much larger (potentially providing a large SERS signal), but this is partly compensated by the fact that coupling to SPPs is reduced. The latter may also prevent a clear measurement of the SPR reflectivity minimum. This approach may therefore be suited to SERS measurements on flat surfaces (which still benefits from the collection of the entire Kretschmann cone), but is not appropriate to combined SPR/SERS microscopy studies.

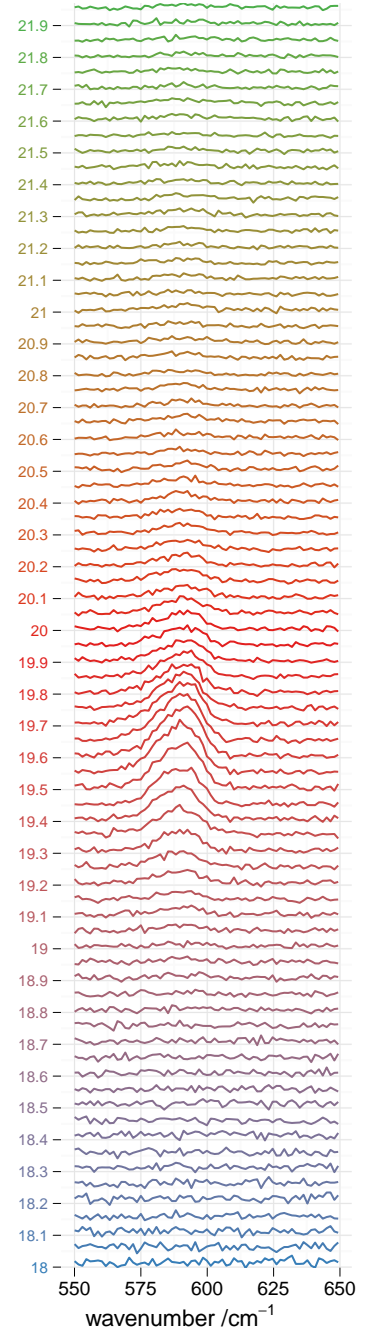

Fig. 56: Measured Raman spectra of NB molecules adsorbed on a Au substrate (in air), illuminated $\left(\lambda_{\mathrm{L}}=\right.$ $633 \mathrm{~nm}$ ) at different incident angles $\alpha^{\text {eff }}$ in the KC depicted in Fig. 50. Spectra are shifted vertically for clarity. The Kretschmann resonance can be clearly seen within a very small angular range.
The exact strategy (or strategies, since combining them could be advantageous in some cases) should be decided on a case-bycase basis. This brief discussion however highlights the importance of being able to adjust spot-size/beam divergence at will in such experiments. This flexibility is therefore an important feature of our proposed set-up.

All the plots presented so far in this section show the integrated Raman intensity of a particular peak across the resonance (i.e. as a function of angle). It is interesting to show the actual raw Raman data for the NB peak at $592 \mathrm{~cm}^{-1}$ across the resonance. Fig. 56 presents the actual Raman data for one of the examples given here (after subtraction of the fluorescence background). A clear angular resonance is seen over the very small angular range $19^{\circ}-20.5^{\circ}$ (cube angle $\alpha$ ) corresponding to the Kretschmann resonance. The data were recorded as a time series sequence, synchronous with the scanning of the (shifter) cube. The connection between the shifter cube angle and the incidence angle is mostly linear up to $\sim 50^{\circ}$ (see Fig. 52) and was fully characterized by both measurements and calculation in Subsection 4.6.3. 


\subsubsection{A platform for analytical SERS studies}

The standard implementation of the Kretschmann configuration with a prism can only achieve a very good coupling to SPPs in some specific circumstances that do not involve high magnification objectives [60]. Furthermore, due to the tilted substrate, it is not possible to scan over the surface of the sample without compromising the focusing condition. In contrast, the coupling scheme proposed in this section offers a real promise of performing SERS microscopy in a convenient platform for analytical studies. Where the use of a prism imposes a natural limitation on how close one can approach the sample - forcing the use of long working distance, low-NA objectives- our SPR configuration uses a conventional TIRF illumination scheme suitable for microscopy with high-NA and high magnification objectives. In terms of performance, only a small fraction of the emitted Kretschmann cone containing the SERS signal from the substrate is collected in the case of prism-coupling. This has been discussed extensively in Section 4.5 (see also Ref. [60]). Comparatively, the present configuration collects the full Kretschmann cone in emission.
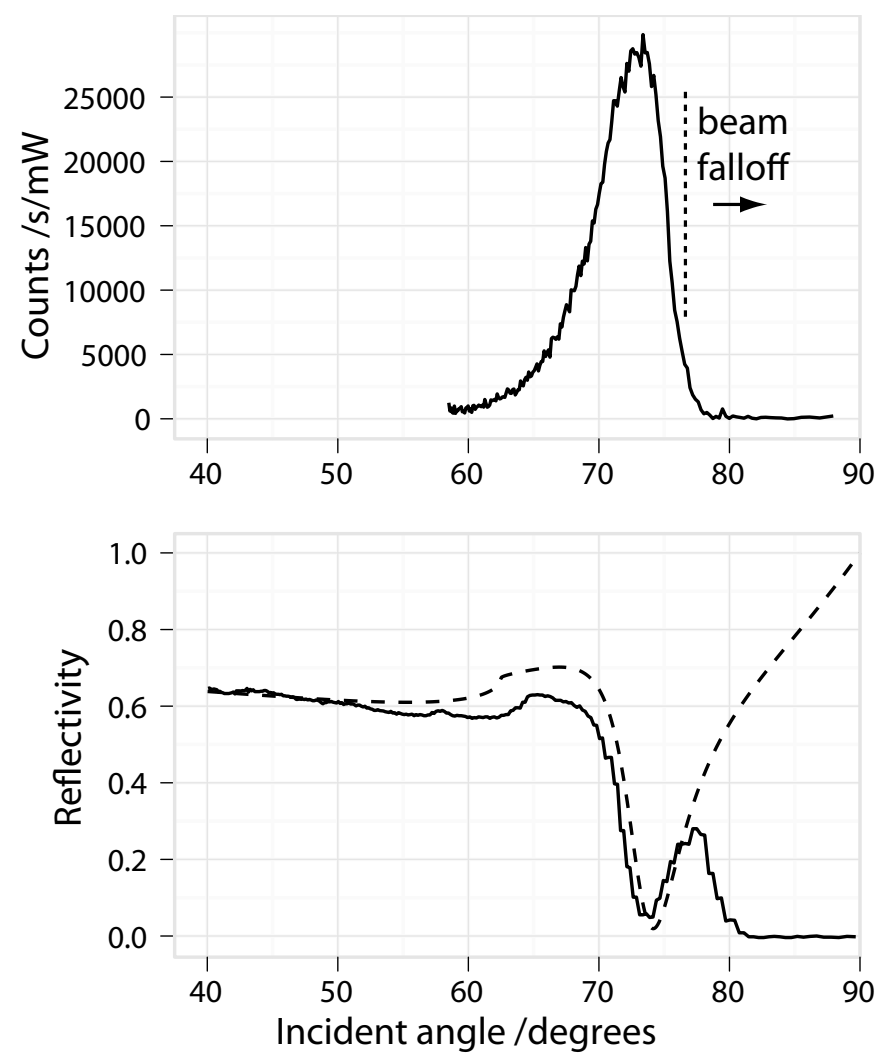

Fig. 57 :

Experimental variation of the reflectivity (bottom) and integrated $595 \mathrm{~cm}^{-1}$ Raman peak of Nile Blue (top). A $5: 1$ beam expander was used to expand the laser beam of wavelength $\lambda=632.8 \mathrm{~nm}$. The substrate consisted in a $48 \mathrm{~nm}$ Au film with a $2 \mathrm{~nm}$ Ti adhesion layer deposited onto a glass substrate with refractive index 1.52. The Au surface was exposed to $N B$ molecules dispersed in water with concentration $100 \mu \mathrm{M}$. The theoretical prediction for the reflectivity is shown with a dashed line. The experiment follows closely the theory until the off-axis beam approaches the edge of the objective, resulting in a beam falloff. The Kretschmann resonance in water is at a very high incidence angle of $\sim 74^{\circ}$, but still achievable with an objective with $N A=1.49$, like the one used in our setup.

In order to illustrate the potential of the proposed configuration to perform applications-driven analytical studies, we present in Fig. 57 a proof-of-principle measurement of the reflectivity and Raman resonance of Nile Blue molecules immersed in water. This is a situation highly relevant to a number of applications, such as biosensing, where working in air is a disadvantage. In a liquid solvent, molecules can flow in the solution; notably it allows the study of fluorescence enhancement which would be mostly quenched in the first layer as studied in dry conditions. The plasmon resonance for the glass/Au/water system is centered around $\sim 74^{\circ}$, close to the limit of the maximum 
angle allowed by the high NA objective. The high, almost grazing angle, together with the relatively "large" beam diameter, results in a sharp drop of the signal when approaching $\sim 80^{\circ}$. Nevertheless, the SPR position is clearly observed in the reflectivity curve, and the Raman signal follows the expected angular resonance until the loss of incident light causes a sharp drop in signal. These results confirm the potential of hybrid SPR-SERS microscopy in a broad range of analytical applications, that might include substrates in air or in water with improved spatial resolution over large areas of uniform enhancement. 


\subsection{Conclusion and outlook}

For a technique that has been overlooked for potential applications in SERS for decades, we believe that the results contained in this chapter present all the key proof-of-principle points to demonstrate that there is a promising way ahead for it. Besides the fact that uniform enhancements over large areas are achieved (a very desirable feature; that we confirmed by doing spatial scans), the main beauty of doing SERS in the Kretschmann configuration relies mainly in its predictability: in the fact that SPPs on flat surfaces represent the simplest plasmonic system and in being more susceptible to predictions and quantifications than standard substrates based on nanotechnology. A series of developments from here are easily envisioned to improve our control further and fulfill the potential of the technique on both fundamental and applied topics.

In Section 4.5 we were able to show that doing SERS in the Kretschmann configuration is compatible with existing spectroscopic platforms for SPRS in liquids (and with Au substrates). The combination of these two techniques opens endless possibilities in both bio-spectroscopy and (at a more fundamental level) the study of surface-enhanced/quenched fluorescence. Clearly the next challenge is to show the performance of the two techniques for non resonant molecules.

In Section 4.6 we present a new way of performing SERS microscopy over flat surfaces with the following main characteristics: $(i)$ a good spatial resolution through the use of a high-NA objective, $(i i)$ improved collection efficiency with respect to typical prism-coupling versions of the Kretschmann configuration, (iii) uniform enhancement over the surface, and (iv) the ability to simultaneously measure the SPR reflectivity. Besides the obvious study of heterogeneous monolayers on surfaces (due to the orientation of domains of molecules, or otherwise), it is possible to envision future applications with functionalized surfaces where hundreds (or possibly thousands) of compounds could be monitored on a single SPR-chip with perfectly uniform enhancement, using the ability to spatially resolve the signals at different places on the surface.

Especially the technique presented in Section 4.6 sets the necessary platform to attempt singlemolecule detection on flat surfaces. The spatial resolution feature is essential in this latter case. As pointed out in the introduction, this technique does not involve any new development in the nanotechnology of the substrates (which might be difficult to replicate and exploit), but rather it is an instrumental development in the way SPPs are excited and the SERS signal collected. It is interesting to realize that since the pioneering work on the coupling of SPPs by Kretschmann [71] and Otto [116] in the late 60's, this is the first time that concept is mingled with SERS microscopy (which has requirements of its own in terms of spot size and collection of the signal). While there are natural limitations to satisfy the requirements of SERS microscopy, it is clear from the results in this chapter that the two techniques can work in a fruitful symbiosis, opening new doors to the applications and use of SERS as an analytical technique. 


\section{Chapter 5}

\section{Overall conclusion}

In Chapter 2 we developed a new, SERS-based technique that can be used (with certain precautions) to measure resonant Raman cross-sections. By measuring those cross-section-values across the visible spectrum for RH6G we were able to establish a proof of principle that the proposed technique actually works. This can be considered a major breakthrough previously considered very difficult: "Resonance Raman scattering ... largely places the experimentalist at nature's mercy. If the Raman spectrum is weak or extremely complicated, or sits on top of a strong fluorescence background, there is not much that can be done about it."(Anne Myers-Kelley in a recent review article [29]). But it is also a very useful result: RH6G in one of the most widely used dyes for which so far only one resonant Raman cross-section was measured and published by a single group using femto-second stimulated Raman spectroscopy [30], a technique available only to a handful of research groups around the world. Our approach on the other hand is simple and accessible for anyone with a Raman spectrometer. It is easy to see the possible impact of this new avenue.

In Chapter 3 we were able to deliver the most direct demonstration of SSR since they were proposed and published by Moskovits in 1982 [13]. We think that this is a significant contribution to the field as it eliminates the uncertainties associated with measurements on complex systems and the concomitant set of assumptions imposed on them. On a practical level we opened up a new avenue to determine the orientation of molecules adsorbed on metallic surfaces by measuring their angle depend Raman-signal on a flat piece of Au. This approach showed excellent agreement between theoretical predictions and experimental results using only a single fitting parameter. Owing to its simplicity this chapter constitutes a textbook like study that allows one to understand local fields on metallic films in general and SSR in particular without the usual interference of complex substrates that require a lot of additional assumptions.

Finally, in Chapter 4, we were able to demonstrate for the first time that SPRS and SERS can be merged into a very useful, hybrid technique that combines the sensitivity of SPRS with the analytical power of SERS. To make this new tool more readily accessible for the scientific community we developed a setup that can be easily implemented in any standard Raman spectrometer and has the additional benefit of allowing one to do microscopy. But this new setup is also a novelty in how to perform SERS on flat metallic films by showing a new, convenient way to implement the Kretschmann configuration. This setup's widespread use has so far been hampered by being rather cumbersome to implement, by prohibiting the collection of all of the available signal (Kretschmann cone) and by being incompatible with microscopic studies that require scanning of 
a surface.

All of the work presented in this thesis was carried out on flat, metallic films. While this choice for a SERS substrate was not mandatory for the work done in Chapter 2 (and some other substrates were used for comparison), the results presented in Chapter 3 and 4 necessitated a flat, metallic film. While the EFs that can be obtained are not as high as with other, more complex substrates (e.g. colloids), their usefulness - both for practical application and fundamental studies - is self-evident. With the results obtained we therefore hope to contribute to a change in paradigm that leads away from the chase for ever more complex and sophisticated substrates to more fundamental studies on what can be argued is the simplest plasmonic system available: a flat, metallic surface! 


\section{References}

[1] M. J. Natan, "Concluding remarks: surface enhanced Raman scattering," Faraday Discussions, vol. 132, pp. 321-328, 2006.

[2] E. C. Le Ru and P. G. Etchegoin, Principles of Surface Enhanced Raman Spectroscopy and Related Plasmonic Effects. Amsterdam: Elsevier, 2009.

[3] M. Fleischmann, P. J. Hendra, and A. J. McQuillan, "Raman spectra of pyridine adsorbed at a silver electrode,” Chem. Phys. Let., vol. 26, pp. 163-166, 1974.

[4] R. F. Aroca, Surface-Enhanced Vibrational Spectroscopy. Chichester: John Wiley \& Sons, 2006.

[5] S. Schluecker, Surface Enhanced Raman Spectroscopy. Weinheim: WILEY-VCH Verlag \& Co. KGaA, 2011.

[6] D. L. Jeanmaire and R. P. V. Duyne, "Surface Raman spectroelectrochemistry: Part i. heterocyclic, aromatic, and aliphatic amines adsorbed on the anodized silver electrode," J. Electroanal. Chem., vol. 84, pp. 1-20, 1977.

[7] M. Moskovits, "Surface-enhanced spectroscopy," Rev. Mod. Phys., vol. 57, no. 3, pp. 783-826, 1985.

[8] E. C. Le Ru, M. Meyer, and P. G. Etchegoin, "Proof of single-molecule sensitivity in surface enhanced Raman scattering (SERS) by means of a two-analyte technique," J. Phys. Chem. B, vol. 110, no. 4, pp. 1944-1948, 2006.

[9] N. P. W. Pieczonka and R. F. Aroca, "Single molecule analysis by surface-enhanced Raman scattering,” Chem. Soc. Rev., vol. 37, pp. 946-954, 2008.

[10] P. G. Etchegoin and E. C. Le Ru, "A perspective on single molecule SERS: Current status and future challenges," Phys. Chem. Chem. Phys., vol. 10, pp. 6079-6089, 2008.

[11] E. Blackie, E. C. Le Ru, M. Meyer, M. Timmer, B. Burkett, P. Northcote, and P. G. Etchegoin, "Bi-analyte SERS with isotopically edited dyes," Phys. Chem. Chem. Phys., vol. 10, pp. 41474153, 2008.

[12] J. A. Dieringer, R. B. Lettan II, K. A. Scheidt, and R. P. Van Duyne, "A frequency domain existence proof of single-molecule surface enhanced Raman spectroscopy," J. Am. Chem. Soc., vol. 129, pp. 16249-16256, 2007.

[13] M. Moskovits, “Surface selection rules,” J. Chem. Phys., vol. 77, pp. 4408-4416, 1982. 
[14] D. A. Long, The Raman Effect, a Unified Treatment of the Theory of Raman Scattering by Molecules. Chichester: John Wiley and Sons Ltd., 2002.

[15] A. Smekal, "Zur Quantentheorie der Dispersion,” Naturwissenschaften, vol. 11, pp. 873-875, 1923.

[16] C. V. Raman and K. S. Krishnan, "A new type of secondary radiation," Nature, vol. 121, pp. 501-502, 1928.

[17] S. A. Maier, Plasmonics, Fundamentals and Applications. Berlin: Springer, 2007.

[18] L. Novotny and B. Hecht, Principles of Nano-Optics. Cambridge: Cambridge University Press, 2006.

[19] P. C. Lee and D. Meisel, "Adsorption and surface-enhanced Raman of dyes on silver and gold sols,” J. Phys. Chem., vol. 86, pp. 3391-3395, 1982.

[20] N. M. B. Perney, J. J. Baumberg, M. E. Zoorob, M. D. B. Charlton, S. Mahnkopf, and C. M. Netti, "Tuning localized plasmons in nanostructured substrates for surface-enhanced Raman scattering," Optics Express, vol. 14, pp. 847-857, 2006.

[21] S. A. Meyer, E. C. Le Ru, and P. G. Etchegoin, "Quantifying resonant Raman cross section with SERS,” J. Phys. Chem. A, vol. 114, pp. 5515-5519, 2010.

[22] H. W. Schroetter and H. W. Kloeckner, Raman Spectroscopy of Gases and Liquids. Berlin: Springer-Verlag, 1979.

[23] W. Demtröder, Laser Spectroscopy. Berlin: Springer, 2002.

[24] E. C. Le Ru, E. Blackie, M. Meyer, and P. G. Etchegoin, "SERS enhancement factors: a comprehensive study,” J. Phys. Chem. C, vol. 111, no. 37, pp. 13794-13803, 2007.

[25] P. J. G. Goulet and R. F. Aroca, "Distinguishing individual vibrational fingerprints: single molecule SERRS from one-to-one binary mixtures in Langmuir-Blodgett monolayers," Anal. Chem., vol. 79, pp. 2728-2734, 2007.

[26] W. P. Ambrose, P. M. Goodwin, J. H. Jett, A. V. Orden, J. H. Werner, and R. A. Keller, "Single molecule fluorescence spectroscopy at ambient temperature," Chem. Rev., vol. 99, pp. 29292956, 1999.

[27] B. Valeur, Molecular Fluorescence. Weinheim: Wiley-VCH, 2007.

[28] C. Galloway, P. G. Etchegoin, and E. C. Le Ru, "Ultrafast nonradiative decay rates on metallic surfaces by comparing surface-enhanced Raman and fluorescence signals of single molecules," Phys. Rev. Lett., vol. 103, pp. 063003-(1-4), 2009.

[29] A. Myers-Kelley, "Resonance Raman and resoanance Hyper-Raman intensities: Structure and dynamics of molecular excited states in solution," J. Phys. Chem. A, vol. 112, pp. 1197511991, 2008.

[30] S. Shim, C. M. Stuart, and R. A. Mathies, "Resonance Raman cross-sections and vibronic analysis of rhodamine 6G from broadband stimulated Raman spectroscopy," ChemPhysChem, vol. 9, pp. 697-699, 2008. 
[31] P. Kukura, D. W. McCamant, and R. A. Mathies, "Femtosecond time-resolved stimulated Raman spectroscopy of the S2 excited state of beta-carotene," J. Phys. Chem. A, vol. 108, pp. 5921-5925, 2004.

[32] S. Y. Lee, D. Zhang, D. W. McCamant, P. Kukura, and R. A. Mathies, "Theory of femtosecond stimulated Raman spectroscopy,” J. Chem. Phys., vol. 121, pp. 3632-3642, 2004.

[33] P. Kukura, S. Yoon, and R. A. Mathies, “Femtosecond stimulated Raman spectroscopy," Anal. Chem., vol. 78, pp. 5952-5959, 2006.

[34] D. W. McCamant, P. Kukura, and R. A. Mathies, "Femtosecond broadband stimulated Raman: A new approach for high-performance vibrational spectroscopy," Appl. Spectrosc., vol. 57, pp. 1317-1323, 2003.

[35] A. C. Benniston, P. Matousek, I. E. McCulloch, A. W. Parker, and M. Towrie, "Detailed picosecond Kerr-gated time-resolved resonance Raman spectroscopy and time-resolved emission studies of merocyanine 540 in various solvents," J. Phys. Chem. A, vol. 107, pp. 4347-4353, 2003.

[36] R. Wilbrandt, "Time-resolved resonance Raman spectroscopy: A retrospect on the early days," Biospectroscopy, vol. 2, pp. 263-275, 1996.

[37] L. Jensen and G. C. Schatz, "Resonance Raman scattering of rhodamine 6G as calculated using time-dependent density functional theory," J. Phys. Chem. A, vol. 110, no. 18, pp. 5973$5977,2006$.

[38] H. Xu, X.-H. Wang, M. P. Persson, H. Q. Xu, M. Käll, and P. Johansson, "Unified treatment of fluorescence and Raman scattering processes near metal surfaces," Phys. Rev. Lett., vol. 93, no. 24, pp. 243002-(1-4), 2004.

[39] G. Ford and W. H. Weber, "Electromagnetic interactions of molecules with metal surfaces," Phys. Rep., vol. 113, pp. 195-287, 1984.

[40] P. Bharadwaj and L. Novotny, "Spectral dependence of single molecule fluorescence enhancement," Opt. Express, vol. 15, pp. 14266-14274, 2007.

[41] E. C. Le Ru, P. G. Etchegoin, J. Grand, N. Félidj, J. Aubard, and G. Lévi, "Mechanisms of spectral profile modification in surface enhanced fluorescence," J. Phys. Chem. C, vol. 111, no. 44, pp. 16076-16079, 2007.

[42] Z. Q. Tian, Z. L. Yang, B. Ren, J. F. Li, Y. Zhang, X. F. Lin, J. W. Hu, and D. Y. Wu, "Surfaceenhanced Raman scattering from transition metals with special surface morphology and nanoparticle shape," Faraday Discuss., vol. 132, pp. 159-170, 2006.

[43] P. G. Etchegoin, P. D. Lacharmoise, and E. C. Le Ru, "Influence of photostability on singlemolecule surface enhanced Raman scattering enhancement factors," Anal. Chem., vol. 81, pp. 682-688, 2009.

[44] P. G. Etchegoin, E. C. Le Ru, R. C. Maher, and L. F. Cohen, "Enhancement factor averaging and the photostability of probes in SERS vibrational pumping," Phys. Chem. Chem. Phys., vol. 9, pp. 4923-4929, 2007. 
[45] R. G. Freeman, R. M. Bright, M. B. Hommer, and M. Natan, "Size selection of colloidal gold aggregates by filtration: Effect on surface-enhanced Raman scattering intensities," J. Raman Spectrosc., vol. 30, pp. 733-738, 1999.

[46] E. C. Le Ru, M. Meyer, E. Blackie, and P. G. Etchegoin, "Advanced aspects of electromagnetic SERS enhancment factors at a hot spot,” J. Raman Spectrosc., vol. 39, pp. 1127-1134, 2008.

[47] S. Buchanan, E. C. Le Ru, and P. G. Etchegoin, "Plasmon-dispersion corrections and constraints for surface selection rules of single molecule SERS spectra," Phys. Chem. Chem. Phys., vol. 11, pp. 7406-7411, 2009.

[48] M. Meyer, E. C. Le Ru, and P. G. Etchegoin, "Self-limiting aggregation leads to long-lived metastable clusters in colloidal solutions,” J. Phys. Chem. B, vol. 110, pp. 6040-6047, 2006.

[49] E. C. Le Ru, S. A. Meyer, C. Artur, P. G. Etchegoin, J. Grand, P. Lang, and F. Maurel, "Experimental demonstration of surface selection rules for SERS on flat metallic surfaces," Chem. Comm., vol. 47, pp. 3903-3905, 2011.

[50] J. Jiang, K. Bosnick, M. Maillard, and L. Brus, "Single molecule Raman spectroscopy at the junctions of large Ag nanocrystals,” J. Phys. Chem. B, vol. 107, no. 37, pp. 9964-9972, 2003.

[51] T. O. Shegai and G. Haran, "Probing the Raman scattering tensors of individual molecules," J. Phys. Chem. B, vol. 110, pp. 2459-2461, 2006.

[52] M. Osawa, N. Matsuda, K. Yoshii, and I. Uchida, "Charge transfer resonance Raman process in surface-enhanced Raman scattering from p-aminothiophenol adsorbed on Ag: HerzbergTeller contribution," J. Phys. Chem., vol. 98, pp. 12702-12707, 1994.

[53] M. Moskovits and J. S. Suh, "Surface selection rules for surface-enhanced Raman spectroscopy: calculations and application to the surface-enhanced Raman spectrum of phthalazine on silver," J. Chem. Phys., vol. 88, pp. 5526-5530, 1984.

[54] M. Moskovits and J. S. Suh, "Surface geometry change in 2-naphthoic acid adsorbed on silver,” J. Phys. Chem., vol. 92, pp. 6327-6329, 1988.

[55] E. C. Le Ru, M. Dalley, and P. G. Etchegoin, "Plasmon resonances of silver colloids studied by surface enhanced Raman spectroscopy,” Curr. Appl. Phys., vol. 6, pp. 411-414, 2006.

[56] E. C. Le Ru, P. G. Etchegoin, J. Grand, N. Félidj, J. Aubard, G. Lévi, A. Hohenau, and J. Krenn, "Surface enhanced Raman spectroscopy on nanolithography-prepared substrates," Curr. Appl. Phys., vol. 8, pp. 467-470, 2008.

[57] N. Karsi, P. Lang, M. Chehimi, M. Delamar, and G. Horowitz, "Modification of indium tin oxide films by alkanethiol and fatty acid self-assembled monolayers: A comparative study," Langmuir, vol. 22, pp. 3118-3124, 2006.

[58] P. G. Etchegoin, E. C. Le Ru, and M. Meyer, "An analytic model for the optical properties of gold," J. Chem. Phys., vol. 125, no. 16, pp. 164705-(1-3), 2006.

[59] H. Kuramitz, K. Sugawara, M. Kawasaki, K. Hasebe, H. Nakamura, and S. Tanaka, "Electrocatalytic reduction of hemoglobin at a self-assembled monolayer electrode containing redox dye, nile blue as an electron-transfer mediator," Anal. Sci., vol. 15, pp. 589-592, 1999. 
[60] S. A. Meyer, E. C. Le Ru, and P. G. Etchegoin, "Combining surface plasmon resonance (SPR) spectroscopy with surface-enhanced Raman scattering (SERS)," Anal. Chem., vol. 83, pp. 2337-2344, 2011.

[61] S. A. Meyer, B. Auguie, E. C. Le Ru, and P. G. Etchegoin, "Combined SPR and SERS microscopy in the Kretschmann configuration,” J. Phys. Chem. A, vol. 116, pp. 1000-1007, 2012.

[62] B. Huang, F. Yu, and R. N. Zare, "Surface plasmon resonance imaging using a high numerical aperture microscope objective," Anal. Chem., vol. 79, no. 7, pp. 2979-2983, 2007.

[63] D. Axelrod, "Total internal reflection fluorescence microscopy in cell biology," Methods in Enzymology, vol. 361, pp. 1-33, 2003.

[64] D. J. O'Shannessy, "Determination of kinetic rate and equilibrium binding constants for macromolecular interactions: a critique of the surface plasmon resonance literature," Curr. Opin. Biotechnol., vol. 5, pp. 65-71, 1994.

[65] M. Malmqvist, "Surface plasmon resonance for detection and measurement of antibodyantigen affinity and kinetics," Curr. Opin. Immunol., vol. 5, pp. 282-286, 1993.

[66] A. Szabo, L. Stolz, and R. Granzow, "Surface plasmon resonance and its use in biomolecular interactions analysis (BIA)," Curr. Opin. Struct. Biol., vol. 5, pp. 699-705, 1995.

[67] R. J. Fisher and M. Fivash, "Surface plasmon resonance based methods for measuring the kinetics and binding affinities of biomolecular interactions," Curr. Opin. Biotechnol., vol. 5, pp. 389-395, 1994.

[68] D. H. Margulies, D. Plaksin, S. Khilko, and M. T. Jelonek, "Studying interactions involving the T-cell antigen receptor by surface plasmon resonance," Curr. Opin. Immunol., vol. 8, pp. 262-270, 1996.

[69] R. J. Stokes, A. Macaskill, J. A. Dougan, P. G. Hargreaves, H. M. Stanford, W. E. Smith, K. Faulds, and D. Graham, "Highly sensitive detection of dye-labelled DNA using nanostructured gold surfaces," Chem. Commun., vol. 27, pp. 2811-2813, 2007.

[70] T. A. Alexander and D. M. Le, "Characterization of a commercialized SERS-active substrate and its application to the identification of intact bacillus endospores," Appl. Optics, vol. 46, pp. 3878-3890, 2007.

[71] E. Kretschmann and H. Raether, "Radiative decay of non-radiative surface plasmons excited by light," Z. Naturforsch, vol. 23A, pp. 2135-2136, 1968.

[72] J. A. Dieringer, A. D. McFarland, N. C. Shah, D. A. Stuart, A. V. Whitney, C. R. Yonzon, M. A. Young, X. Zhang, and R. P. V. Duyne, "Surface enhanced Raman spectroscopy: new materials, concepts, characterization tools, and applications," Faraday Discussions, vol. 132, pp. 9-26, 2006.

[73] M. Moskovits, "Surface-enhanced Raman spectroscopy: a brief retrospective," J. Raman Spectrosc., vol. 36, no. 6-7, pp. 485-496, 2005.

[74] W. E. Smith, "Practical understanding and use of surface enhanced Raman scattering/surface enhanced resonance Raman scattering in chemical and biological analysis," Chem. Soc. Rev., vol. 37, pp. 955-964, 2008. 
[75] C. L. Nehl, H. Liao, and J. H. Hafner, "Optical properties of star-shaped gold nanoparticles," Nano Letters, vol. 6, pp. 683-688, 2006.

[76] D. Graham, D. G. Thompson, W. E. Smith, and K. Faulds, "Control of enhanced Raman scattering using a DNA-based assembly process of dye-coded nanoparticles," Nature Nanotech. vol. 3, pp. 548-551, 2008.

[77] R. W. Taylor, T.-C. Lee, O. A. Scherman, R. Esteban, J. Aizpurua, F. M. Huang, J. J. Baumberg, and S. Mahajan, "Precise subnanometer plasmonic junctions for SERS within gold nanoparticle assemblies using cucurbit[n]uril "glue”," ACS Nano, vol. 5, pp. 3878-3887, 2011.

[78] M. Green and F. M. Liu, "SERS substrates fabricated by island lithography: the silver/pyridine system," J. Phys. Chem. B, vol. 107, pp. 13015-13021, 2003.

[79] C. L. Haynes and R. P. V. Duyne, "Nanosphere lithography: A versatile nanofabrication tool for studies of size-dependent nanoparticle optics,” J. Phys. Chem. B, vol. 105, pp. 5599-5611, 2001.

[80] C. Noguez, "Surface plasmons on metal nonoparticles: The influence of shape and physical environment," J. Phys. Chem. C, vol. 111, pp. 3806-3819, 2007.

[81] A. F. McCabe, C. Eliasson, R. A. Prasath, A. Hernandez-Santana, L. Stevenson, I. Apple, P. A. G. Cormack, D. Graham, W. E. Smith, P. Corish, S. J. Lipscomb, E. R. Holland, and P. D. Prince, "SERRS labeled beads for multiplex detection," Faraday Discussions, vol. 132, pp. 303-308, 2006.

[82] D. Graham, C. McLaughlin, G. McAnally, J. C. Jones, P. C. White, and W. E. Smith, "Synthesis of novel monoazo benzotriazole dyes specifically for surface enhanced resonance Raman scattering," Chem. Comm., pp. 1187-1188, 1998.

[83] D. K. Lim, K. S. Jeon, H. M. Kim, J. M. Nam, and Y. D. Suh, "Nanogap-engineerable Ramanactive nanodumbbells for single-molecule detection," Nature Mat., vol. 9, pp. 60-67, 2010.

[84] S. L. Kleinman, E. Ringe, N. Valley, K. L. Wustholz, E. Phillips, K. A. Scheidt, G. C. Schatz, and R. P. Van Duyne, "Single-molecule surface-enhanced Raman spectroscopy of crystal violet isotopologues: Theory and experiment," J. Am. Chem. Soc., vol. 133, pp. 4115-4122, 2011.

[85] P. G. Etchegoin and E. C. Le Ru, "Resolving single molecules in surface-enhanced Raman scattering within the inhomogeneous broadening of Raman peaks," Anal. Chem., vol. 82, pp. 2888-2892, 2010.

[86] S. M. Stranahan and K. A. Willets, "Super-resolution optical imaging of single-molecule SERS hot spots,” Nano Lett., vol. 10, pp. 3777-3784, 2010.

[87] E. Cortés, P. G. Etchegoin, E. C. Le Ru, A. Fainstein, M. Vela, and R. Salvarezza, "Monitoring the electrochemistry of single molecules by surface-enhanced Raman spectroscopy," J. Am. Chem. Soc., vol. 132, pp. 18034-18037, 2010.

[88] D. P. dos Santos, G. F. Andrade, M. L. A. Temperini, and A. G. Brolo, "Electochemical control of the time-dependent intensity fluctuations in surface-enhanced Raman scattering (SERS)," J. Phys. Chem. C, vol. 113, pp. 17737-17744, 2009. 
[89] D. R. Ward, N. J. Halas, J. W. Ciszek, J. M. Tour, Y. Wu, P. Nordlander, and D. Natelson, "Simultaneous measurements of electronic conduction and Raman response in molecular junctions.," Nano Lett., vol. 8, pp. 919-924, 2008.

[90] P. G. Etchegoin, M. Meyer, E. Blackie, and E. C. Le Ru, "Statistics of single-molecule surface enhanced Raman scattering signals: fluctuation analysis with multiple analyte techniques," Anal. Chem., vol. 79, pp. 8411-85415, 2007.

[91] V. P. Drachev, M. D. Thoreson, V. Nashine, E. N. Khaliullin, D. Ben-Amotz, V. J. Davisson, and V. M. Shalaev, "Adaptive silver films for surface-enhanced Raman spectroscopy of biomolecules," J. Raman Spectrosc., vol. 36, pp. 648-656, 2005.

[92] B. Vlckova, M. Moskovits, I. Pavel, K. Siskova, M. Sladkova, and M. Slouf, "Single-molecule surface-enhanced Raman spectroscopy from a molecularly-bridged silver nanoparticle dimer," Chem. Phys. Lett., vol. 455, pp. 131-134, 2008.

[93] J. B. Jackson, S. L. Westcott, L. R. Hirsch, J. L. West, and N. J. Halas, "Controlling the surface enhanced Raman effect via the nanoshell geometry,” Appl. Phys. Lett., vol. 82, pp. 257-259, 2003.

[94] J. Hu, B. Zhao, W. Xu, Y. Fan, B. Li, and Y. Ozaki, "Simple method for preparing controllably aggregated silver particle films used as surface-enhanced Raman scattering active substrates," Langmuir, vol. 18, pp. 6839-6844, 2002.

[95] M. Fan and A. G. Brolo, "Silver nanoparticles self assembly as SERS substrates with near single molecule detection limit," Phys. Chem. Chem. Phys., vol. 11, no. 34, pp. 7381-7389, 2009.

[96] E. Kretschmann, "Die Bestimmung optischer Konstanten von Metallen durch Anregungen von Oberflächenplasmaschwingungen,” Z. Phys., vol. 241, pp. 313-324, 1971.

[97] G. Sabatte, R. Keir, M. Lawlor, M. Black, D. Graham, and W. Smith, "Comparison of surfaceenhanced resonance Raman scattering and fluorescence for detection of a labeled antibody," Anal. Chem., vol. 80, no. 7, pp. 2351-2356, 2008.

[98] C. J. L. Constantino, T. Lemma, P. A. Antunes, and R. Aroca, "Single-molecule detection using SERRS and Langmuir-Blodgett monolayers," Anal. Chem., vol. 73, pp. 3674-3678, 2001.

[99] A. Otto, Surface-enhanced Raman scattering: classical and chemical origins. Berlin: Springer-Verlag, 1984.

[100] W. Wittke, A. Hatta, and A. Otto, "Efficient use of the surface plasmon polariton resonance in light scattering from adsorbates," App. Phys. A, vol. 48, pp. 289-294, 1989.

[101] Y. J. Chen, W. B. Chen, and E. Burstein, "Surface-electromagnetic-wave-enhanced Raman scattering by overlayers on metals," Phys. Rev. Lett., vol. 36, pp. 1207-1210, 1976.

[102] B. Pettinger, A. Tadjeddine, and D. M. Kolb, "Enhancement in Raman intensity by use of surface plasmons," Chem. Phys. Lett., vol. 66, pp. 544-548, 1979.

[103] R. Dornhaus, R. E. Benner, and R. K. Chang, "Surface plasmon contribution to SERS," Surf. Sci., vol. 101, pp. 367-373, 1980. 
[104] H. W. K. Tom, C. K. Chen, A. R. B. de Castro, and Y. R. Shen, "Effect of extended surface plasmons on surface enhanced Raman scattering," Solid State Commun., vol. 41, pp. 259262, 1982.

[105] S. Ushioda and Y. Sasaki, "Raman scattering mediated by surface-plasmon polariton resonance,” Phys. Rev. B, vol. 27, pp. 1401-1404, 1983.

[106] R. M. Corn and M. R. Philpott, "Surface plasmon-enhanced Raman scattering at thin silver films,” J. Chem. Phys., vol. 80, pp. 5245-5249, 1984.

[107] K. Kurosawa, R. M. Pierce, and S. Ushioda, "Raman scattering an attenuated-total-reflection studies of surface-plasmon polaritons," Phys. Rev. B, vol. 33, pp. 789-798, 1986.

[108] J. Giergiel, C. E. Reed, and J. C. Hemminger, "Surface plasmon polariton enhancement of Raman scattering in Kretschmann geometry,” J. Phys. Chem., vol. 92, pp. 5357-5365, 1988.

[109] S. Byahut and T. E. Furtak, "A device for performing surface-plasmon-polariton-assisted Raman scattering from adsorbates on single-crystal silver surfaces," Rev. Sci. Instrum., vol. 61, pp. 27-32, 1990.

[110] M. Futamata, "Surface plasmon polariton enhanced Raman scattering from adsorbates on a "smooth" metal surface: The effect of thickness and dielectric properties of constituents," Langmuir, vol. 11, pp. 3894-3901, 1995.

[111] M. Futamata, E. Keim, A. Bruckbauer, D. Schumacher, and A. Otto, "Enhanced Raman scattering from copper phthalocyanine on Pt by use of Weierstrass prism," Appl. Surf. Sci., vol. 100/101, pp. 60-63, 1996.

[112] M. Futamata, "Application of attenuated total reflection surface-plasmon-polariton Raman spectroscopy to gold and copper," Appl. Opt., vol. 36, pp. 364-375, 1997.

[113] A. Brioude, F. Lequevre, J. Mugnier, J. Dumas, G. Guiraud, and J. C. Plenet, "Raman spectroscopy of sol-gel ultrathin films enhanced by surface plasmon polaritons," J. App. Phys., vol. 88, pp. 6187-6191, 2000.

[114] K. Critchley, E. M. Cheadle, H. L. Zhang, K. J. Baldwin, Q. Liu, Y. Cheng, H. Fukushima, T. Tamaki, D. N. Batchelder, R. J. Bushby, and S. D. Evans, "Surface plasmon Raman scattering studies of liquid crystal anchoring on liquid-crystal based self-assembled monolayers," J. Phys. Chem. B, vol. 113, pp. 15550-15557, 2009.

[115] Y. Liu, S. Xu, B. Tang, Y. Wang, J. Zhou, X. Zheng, B. Zhao, and W. Xua, "Simultaneous measurement of surface plasmon resonance and surface-enhanced Raman scattering," Rev. Sci. Instrum., vol. 81, pp. 036105-(1-3), 2010.

[116] A. Otto, "Excitation of nonradiative surface plasma waves in silver by method of frustrated total reflection,” Z. Phys., vol. 216, no. 4, pp. 398-410, 1968.

[117] D. Axelrod, "Total internal reflection fluorescence microscopy," in Optical Imaging and Microscopy, Springer Series in Optical Sciences (P. Török and F. J. Kao, eds.), Berlin: Springer Verlag, 2007. 
[118] F. D. Stefani, K. Vasilev, N. Bocchio, N. Stoyanova, and M. Kreiter, "Surface-plasmonmediated single-molecule fluorescence through a thin metallic film," Phys. Rev. Lett., vol. 94, pp. 023005-(1-4), 2005.

[119] J. Villatoro and A. Garcia-Valenzuela, "Sensitivity of optical sensors based on laser-excited surface-plasmon waves,” Appl. Optics, vol. 38, pp. 4837-4844, 1999. 